\title{
Modelos multidimensionais da TRI com distribuições assimétricas para os traços latentes
}

\author{
Gilberto da Silva Matos \\ Tese APRESEntada \\ $\mathrm{AO}$ \\ Instituto DE MATEMÁticA E EstatísticA \\ DA \\ Universidade DE SÃo PAUlo \\ PARA \\ OBTENÇÃO DO TÍTULO \\ $\mathrm{DE}$ \\ Doutor em Cî̂NCIAS \\ Programa: Estatística \\ Orientador: Prof. Dr. Heleno Bolfarine \\ Co-Orientador: Prof. Dr. Dalton Francisco de Andrade
}

Durante parte do desenvolvimento deste trabalho o autor recebeu auxílio financeiro da CAPES através do Programa PICDT

São Paulo, dezembro de 2008 


\section{Modelos multidimensionais da TRI com distribuições assimétricas para os traços latentes}

Este exemplar corresponde à redação

final da tese devidamente corrigida e defendida por Gilberto da Silva Matos

e aprovada pela Comissão Julgadora.

Banca Examinadora:

- Prof. Dr. Heleno Bolfarine (orientador) - IME-USP

- Prof. Dr. Dalton Francisco de Andrade (co-orientador) - UFSC

- Prof. Dr. Rosangela Helena Loschi - UFMG

- Prof. Dr. Reynaldo Fernandes - INEP/MEC

- Prof. Dr. Jorge Luiz Bazán Guzmán - PUC-Peru / Chile 
"Ainda que eu falasse as línguas dos homens e dos anjos, se não tivesse amor, seria como o bronze que soa ou como o címbalo que retine. Ainda que tivesse o dom da profecia e conhecesse todos os mistérios e toda a ciência; ainda que tivesse toda a fé, a ponto de transportar montanhas, se não tivesse amor, nada seria. E ainda que distribuísse todos os meus bens aos famintos, ainda que entregasse o meu corpo às chamas, se não tivesse amor, nada disso me adiantaria.

O amor é paciente, é prestativo, não é invejoso, não se ostenta, não se incha de orgulho. Nada faz de inconveniente, não procura o próprio interesse, não se irrita, não guarda rancor. Não se alegra com a injustiça, mas regozija com a verdade. Tudo desculpa, tudo crê, tudo espera, tudo suporta"

(I Coríntios 13:1-7). 
À minha amada esposa Regeli, $e$ aos meus lindos e amados filhos Gabriel Max e Samuel Lucas 


\section{Agradecimentos}

Sem dúvida alguma, toda honra e gratidão é dedicada à Deus pois o amor revelado pelo sacrifício de seu filho amado Jesus Cristo tem o poder de salvar a todos que nele crêem.

Agradeço a Deus pelo seu infinito amor e por me abençoar com a companhia e amizade de tantas pessoas que até mesmo através de atos simples da vida contribuíram para que eu chegasse ao fim deste trabalho. Em especial, gostaria de agradecer:

- Aos meus orientadores Prof. Heleno Bolfarine e Prof. Dalton de Andrade por confiarem e me apoiarem na proposta e desenvolvimento deste trabalho.

- À Profa. Márcia D’Elia Branco, ao Prof. Jorge Bazán e ao amigo Caio Naberezny por se disporem à discutir sobre alguns pontos críticos da tese como, por exemplo, identificabilidade bayesiana.

- Ao Grande amigo Prof. João Maurício pois nunca esqueço do dia em que eu, aos 18 anos e quase um Índio do Amapá, chegava pela primeira vez no Campus do Pici UFC (Ceará) para ir à Coordenação do Curso de Estatística. Naquele dia, passei por um homem que assim que me viu identificou o Gilberto, declarou estar preocupado com alguém que vinha de tão longe, perguntou se tinha chegado de barco e quantos jacarés tinha precisado passar por cima pois eu já tinha perdido uma semana de aula e por tudo isso ele estava me esperando. Me sentí em casa, ele me conduziu à sua sala de Coordenação, tomamos um cafézinho e batemos um longo papo e, a partir dalí, comecei a conhecer este Grande homem. Professor Maurício, te agradeço fortemente por ser um Amigo, um Professor e um Pai para todos nós. Que Deus o abençoe, Sempre!

- Aos Professores e colegas de trabalho da Unidade Acadêmica de Matemática e Estatística (UAME) da UFCG - Campina Grande, Antonio José e Francisco de Souza (Chico) por sempre confiarem e apoiarem a realização deste trabalho. Em especial, gostaria de agradecer a paciência e aos conhecimentos compartilhados pelo Prof. Chico durante os seminários de Probabilidade antes de meu ingresso no Doutorado em Estatística na Universidade de São Paulo. Agradeço, ainda, ao Prof. Claudianor pelas suas excelentes aulas de Teoria da Medida que me foram concedidas assistir. 
- Ao Prof. Luiz Mendes, também colega de trabalho da UAME-UFCG, que num momento de decisão difícil me incentivou para, mesmo sem a certeza de obter uma bolsa de auxílio financeiro, me mudasse juntamente com minha família para São Paulo e cursasse o doutorado. Pois, num futuro próximo, tudo valeria à pena!

- À todos os colegas e amigos que compartilharam momentos de ansiedades, preocupações e também alegrias neste período de doutoramento.

Em especial, gostaria de agradecer à Raydonal Ospina que com sua solidariedade sempre me ajudou quando precisava, principalmente quando decidí comprar um computador na Santa Ifigênia que pudesse levantar vôo e depois aprender a trabalhar com o sistema computacional Linux. Companheiro! Que Deus o abençoe!

Às minhas queridas amigas Gladys Salcedo e Luz Mery. Vocês lebram que durante um período nos denominamos Probessores porque éramos Professores cursando o doutorado sem bolsa de auxílio financeiro em São Paulo? Ha! como é bom lembrar de nossos momentos de estudo nas salinhas do Bloco B do IME-USP. Com vocês, as dificuldades das disciplinas diminuíam.

Aos demais colegas de estudo e amizade: Juvêncio, Patrícia Espinheiro, Michelli, Nelson, Rogério Porto, Caio e demais colegas que peço desculpas se não os citei aqui.

Ao Ronald e sua linda família, Francilene e Marcel. Obrigado por me receber em sua casa durantes os meses que fiquei distante de minha também linda família. A paciência, o carinho e o amor de vocês com certeza me concederam dias felizes.

Ao Pastor Sebastião e sua Esposa Vânia que tenho certeza foram perseverantes em suas Orações, juntamente com muitos irmãos de nossa Primeira Igreja Batista de Campina Grande - PB.

Aos vários amigos que constituímos durante nossa moradia em São Paulo. Em especial, à Marilene, Abraão e toda família. Ao Jorge, Ana e filhos. À tia Dita que tanto carinho tem por meus filhos Samuel e Gabriel. E à tantos outros amigos e parentes que temos semelhante amor e carinho.

- À todos Professores e Funcionários da UAME-UFCG que sempre acreditaram e me apoiaram para que este trabalho pudesse ser realizado com Sucesso. Em especial, agradeço à todos os Professores da Àrea de Estatística e em, particular, à antes colega de doutorado e agora colega de trabalho Michelli que tanto me apoio no período final do doutorado. Agradeço também à nossa destemida colega Patrícia Leal e à Mãe de Michelli, Sra. Maria das Dores. 
- À todos que compoem o Doutorado em Estatística IME-USP. Em particular, à todos que compoem a equipe da CPG que sempre me receberam com coordialidade e paciência. Agradeço, em especial, à Patrícia que assim que cheguei no doutorado me ajudou a melhor me orientar nas Ruas de São Paulo e Osasco e ao Pinho por sempre estar disposto a ajudar.

- À CAPES que através do Programa PICDT financiou parte do programa de doutorado.

- Não poderia de deixar de agradecer à toda família de minha esposa que passei a conhecer e a amar. Ao Alexandre e minha cunhada Jane, juntamente com suas filhas, ao Sr. Ubirajara e D. Marta. À minha cunhada que é uma graça, alegre e divertida, ..., É você mesmo mulher! Você que é mais conhecida por Tia Lalá, a Laíce. Um grande abraço aos meus companheiros montadores de guarda-roupas: Louro, Jeová e Nêne. Um abraço também à Regina e à Lindoura.

- Certamente, sou eternamente grato à Deus pela vida de meus Pais, Avós, Tias e primas. Sou um homem abençoado pois não me faltaram e nem me faltam Mães e Pais. Obrigado Mãe Esmeralda, Pai Manoel, Mãe-Tia Ivone, e en Memorian Mãe-Vó Pastora, Pai-Vô Ediberto e Tia Nazaré. Obrigado às minhas primas e irmãs Larissa e Letície e ao meu Irmão biológico Edvandro. Mano, te Amo! E que Deus esteja sempre com você e sua família Neuza e Nícolas.

- Finalmente, agradeço àqueles que sempre estiveram ao meu lado. Em determinados momentos pacientes e em outros não, em determinados momentos felizes e em outros nem tanto. Mas assim é a vida e sem se importarem como, estiveram sempre ao meu lado, morando em várias cidades deste Brasil: Fortaleza, Recife, Campina Grande, São Paulo e de volta à Campina Grande, sem contar os vários Bairros em que moramos nestas cidades... . Esta é a nossa história e sem vocês eu não saberia o que fazer pois nada é melhor do que constituir e viver o amor de uma Família. Obrigado meu Deus, tudo devo a tí pelos Meus Amores! Esposa Regeli e filhos Gabriel Max e Samuel Lucas, Amo vocês! 


\section{Resumo}

A falta de alternativas ao modelo normal uni/multivariado já é um problema superado pois atualmente é possível encontrar inúmeros trabalhos que introduzem e desenvolvem generalizações da distribuição normal com relação à assimetria, curtose e/ou multimodalidade (Branco e Arellano-Valle (2004), Genton (2004), Arellano-Valle et al. (2006)). No contexto dos modelos unidimensionais da Teoria da Resposta ao Item (TRI), Bazán (2005) percebeu esta realidade e introduziu uma classe denominada PANA (Probito Assimétrico - Normal Assimétrica) a qual permite modelar possíveis comportamentos assimétricos de um modelo (uma probabilidade) de resposta ao item bem como a especificação de uma distribuição normal assimétrica para os traços latentes (unidimensionais) a qual é utilizada no processo de estimação. Motivado pela necessidade de melhor representar os fenômenos da área psicométrica (Heinen, 1996, p. 105) e da atual disponibilidade de distribuições elípticas assimétricas cujas propriedades são tão convenientes quanto aquelas devidas à distribuição normal, a proposta do presente trabalho é apresentar uma extensão do modelo K-dimensional de 3 Parâmetros Probito (Kd3PP) com vetores de traços latentes normalmente distribuídos para o caso t-Assimétrico, gerando, assim, o que denominamos modelo Kd3PP-tA. Nossa proposta, portanto, pode ser considerada como uma extensão do trabalho desenvolvido por Bazán (2005) tanto no sentido de extender a distribuição unidimensional assimétrica dos traços latentes para o caso multidimensional quanto no que conscerne em considerar o achatamento (curtose) da distribuição. Nossa proposta também pode ser vista como uma extensão do trabalho de Béguin e Glas (2001) no sentido de desenvolver o método de estimação bayesiana dos modelos multidimensionais da TRI via DAGS (Dados Aumentados com Amostrador de Gibbs) para o caso em que os vetores de traços latentes comportam-se segundo uma distribuição multivariada t-Assimétrica. No desenvolvimento deste trabalho nos deparamos com uma das principais dificuldades encontradas no processo de estimação e inferência dos modelos multidimensionais da TRI que é a falta de identificabilidade e, com a intenção de ampliar e desmistificar nossos conhecimentos sobre um assunto ainda pouco explorado na literatura da TRI, apresentamos um estudo bibliográfico sobre este tema tanto sob o contexto da inferência clássica quanto bayesiana. Com o intuito de identificar situações particulares em que o uso de uma distribuição normal assimétrica para os traços latentes seja de maior relevância para a estimação e inferência dos parâmetros de item, bem como outros parâmetros relacionados

à distribuição dos traços latentes, algumas análises sobre conjuntos de dados simulados 
são desenvolvidas. Como conclusão destas análises, podemos dizer que há uma melhora superficial quando a informação sobre uma possível assimetria na distribuição dos traços latentes não é ignorada. Além disso, os resultados favoreceram a seleção dos modelos que consideram distribuições assimétricas para os traços latentes, principalmente quando são considerados os modelos que possibilitam a estimação dos parâmetros de localização e escala da distribuição dos vetores de traços latentes. Duas principais contribuições que consideramos de ordem prática, são: a análise e a interpretação de testes através da estimação de modelos uni e multidimensionais da TRI que consideram tanto distribuições simétricas quanto assimétricas para os vetores de traços latentes e a disponibilização de uma função escrita em códigos $R$ e $C++$ para a estimação dos modelos apresentados e desenvolvidos no presente trabalho. 


\section{Abstract}

The lack of alternatives to the univariate or multivariate normal model has been already solved because actually it has been possible to find several works that introduce and develop generalizations of the normal distribution in relation to the asymmetry, kurtosis and/or multimodality (Branco e Arellano-Valle (2004), Genton (2004), Arellano-Valle et al. (2006). In the context of unidimensional models of the Item Response Theory (IRT), Bazán (2005) observed this fact and introduced a class called PANA (Probito Assimétrico - Normal Assimétrica) which allows to take account for asymmetry in the shape of an item response model (probability) and the specification of a skew normal distribution for unidimensional latent traits which is used in the estimation process. Motivated by the need to better represent the phenomenon of psychometric area (Heinen, 1996, p. 105) and the current availability of skew elliptical distributions whose properties are as convenient as those due to normal distribution, the proposal of this work is to provide an extension of Kdimensional 3 Parameters Probit model (Kd3PP) where latent traits vectors are normally distributed for the case of Skew-t distribution (Sahu et al., 2003), generating therefore what we call Kd3PP-St model. Our proposal, therefore, can be regarded as an extension of the work of Bazán (2005) in two ways: the first is extending the unidimensional skew normal distribution of latent traits to the multidimensional case and second in the sense to consider the flattening (kurtosis) of this distribution. Our proposal can also be seen as an extension of the work of Béguin e Glas (2001) in the sense that we develop the Bayesian estimation method of the 3 parameters multidimensional item response model by DAGS (Augmentated Data with Gibbs sampling) for the case where the latent trait vectors behave according to a Skew-t multivariate distribution. In the development of this work we come across one of the main difficulties encountered in the process of estimation and inference of multidimensional IRT models which is the lack of identifiabilitie and, with the intent to demystify and expand our knowledge on a subject still little explored in the literature of the IRT, we present a bibliographical study on this subject both in the context of classical and Bayesian inference. In order to identify particular situations where the use of a skew normal distribution is more relevant to the estimation and inference of item parameters as well as other parameters related to the distribution of latent traits, some analyses on simulated data sets are developed. As results of these analyses, we can say that there is a modest improvement when information about a possible asymmetry in the distribution of latent traits is not ignored. Moreover, the results favored the selection 
of models that consider asymmetric distributions for latent traits, especially when models that enable the estimation of parameters of location and scale from this distribution are considered. Two main contributions that we consider of pratical interest are: analysis and interpretations of tests using unidimensional and multidimensional IRT models that consider both simetric and skewed distributions for the vectors of latent traits and a function written in $R$ and $C++$ language program that is made disponible for the estimation of models treated in this work.

Keywords: Multidimensional Item Response Theory (IRT) models, Multivariate Skewt distribution, Augmentated Data with Gibbs sampling, Identifiabily, Multidimensional equating, $R$ and $C++$ language program. 


\section{Conteúdo}

$\begin{array}{ll}\text { Agradecimentos } & \text { iii }\end{array}$

Resumo vi vi v v v

Abstract $\quad$ viii

1 Introdução 1

1.1 Introdução . . . . . . . . . . . . . . . . . . . . . . . 1

1.2 Objetivos e Organização da Tese . . . . . . . . . . . . . . . 3

2 Modelos Multidimensionais da TRI $\quad 6$

2.1 Modelos Multidimensionais da TRI . . . . . . . . . . . . . . . 6

2.1.1 Sobre a Ausência de Dados . . . . . . . . . . . . . . . . . . . 7

2.1 .2 Suposições . . . . . . . . . . . . . . . . . . . . . 8

2.1.3 Função de Verossimilhança . . . . . . . . . . . . . . . . . . . . . . . 10

2.2 Métodos de Inferência . . . . . . . . . . . . . . . . . . . . 10

2.2.1 Estimação por Máxima Verossimilhança - MV . . . . . . . . . . . . . 11

2.2.2 Inferência por MVM-EM, Bayesiana-EM e Bayesiana via Métodos MCMC 13

2.3 Metodologia Inferencial Adotada . . . . . . . . . . . . . . . . . . . 16

3 Modelos K-dimensionais da TRI e o Problema da Falta de Identificabilidade 18

3.1 Introdução . . . . . . . . . . . . . . . . . . . . . . 18

3.2 Modelos K-dimensionais com Função de Resposta ao Item Probito . . . . . . 19 
3.2.1 Modelo K-dimensional de 2 Parâmetros Probito (Kd2PP) ..... . 19

3.2.2 Modelo K-multivariado de 2 Parâmetros Probito (Km2PP) . . . . . . . . . 21

3.2.3 Modelo K-dimensional de 3 Parâmetros Probito (Kd3PP) ....... 22

3.3 O Problema da Falta de Identificabilidade . . . . . . . . . . . . . . . . . 22

3.3.1 Não-Identificabilidade Sob o Ponto de Vista da Inferência Clássica ... 24

3.3.1.1 Traços Latentes como Efeitos Fixos . . . . . . . . . . . . . 24

3.3.1.2 Traços Latentes como Efeitos Aleatórios . . . . . . . . . . . . 25

3.3.2 Não-Identificabilidade Sob o Ponto de Vista da Inferência Bayesiana . . 28

3.3.2.1 Prioris Impróprias e Verossimilhanças Não-Identificáveis . . . . . . . . 31

3.4 Considerações sobre o Capítulo . . . . . . . . . . . . . . . . . . 32

4 Modelo Kd3PP (e Km3PP) com Distribuições Assimétricas para os Traços Latentes 34

4.1 Introdução . . . . . . . . . . . . . . . . . . . . . 34

4.2 Estimação Bayesiana do Modelo Kd3PP-tA (e Km3PP) via Método DAGS . 35

4.2.1 Verossimilhança Aumentada . . . . . . . . . . . . . . 35

4.2.2 Distribuição t-Assimétrica para os Traços Latentes e Especificação de Distribuições a Priori para

4.2.3 Densidade a Posteriori Aumentada . . . . . . . . . . . . . . . . 41

4.2 .4 Implementação do Algoritmo DAGS . . . . . . . . . . . . . . . . . . . 42

4.2.4.1 Escolha de Valores Iniciais . . . . . . . . . . . . . . . . . . . . . 47

4.2.4.2 Estimação de Sub-Modelos . . . . . . . . . . . . . . . . . 48

4.2.4.3 Metropolis-Hastings e Distribuições Discretas . . . . . . . . . . . . . . 51

4.2.4.4 Extensão do Algoritmo para Modelos de Grupos Múltiplos . . . . . . . 51

4.2.4.5 Implementação Computacional . . . . . . . . . . . . . . . . . . . . 51

5 Análises Envolvendo Dados Simulados $\quad 53$

5.1 Introdução . . . . . . . . . . . . . . . . . . . . . . 53

5.2 Dados Simulados 1: Teste Unidimensional . . . . . . . . . . . . . 54

5.2 .1 Distribuição dos traços latentes . . . . . . . . . . . . . . . 56

5.2 .2 Especificação das Prioris e Identificabilidade dos Modelos . . . . . . . . . 57

Matos, G. S.

$\mathrm{IME} / \mathrm{USP}$ 
5.2.3 Análise de Convergência das Cadeias de Markov . . . . . . . . . . . . . . . 58

5.2.4 Análise das Estimativas Obtidas sob os Modelos 1d2PPN e 1d2PPSn . . . 60

5.2.5 Análise das Estimativas Obtidas sob as Métricas Induzidas pelos Modelos 1d2PPN e 1d2PPSn

5.2 .6 Seleção de Modelo . . . . . . . . . . . . . . . . . . . . . . 68

5.2 .7 Considerações Adicionais e Conclusão . . . . . . . . . . . . . . . . 68

5.3 Dados Simulados 2: Teste Bidimensional _ . . . . . . . . . . . . 70

5.3.1 Características dos Verdadeiros Parâmetros dos Itens e Traços Latentes . . 70

5.3.2 Modelos e Metodologia para as Análises . . . . . . . . . . . . . . . . 72

5.3.3 Informações e Resultados sob os modelos 2d2PPN e 2d2PPSn . . . . . . . 74

5.3.3.1 Sobre as Prioris e a Identificabilidade dos Modelos . . . . . . . . . . 74

5.3.3.2 Sobre a Amostra a Posteriori Simulada. . . . . . . . . . . . . . 75

5.3.3.3 Análise sobre a Acurácia das Estimativas . . . . . . . . . . . . . 75

5.3.3.4 Análise das Estimativas Rotacionadas e Transladadas . . . . . . . . . 77

5.3.4 Informações e Resultados sob os modelos $2 \mathrm{~m} 2 \mathrm{PPN}$ e $2 \mathrm{~m} 2 \mathrm{PPSn}$. . . . . . 84

5.3.4.1 Sobre a Amostra a Posteriori Simulada . . . . . . . . . . . . . . 84

5.3.4.2 Sobre as Distribuições a Priori e os Valores Iniciais para o Algoritmo DAGS 86

5.3.4.3 Análise Comparativa entre os Modelos 2m2PPN e 2m2PPSn . . . . . 87

5.3.4.4 Sobre a Identificabilidade dos Modelos 2m2PPN e 2m2PPSn . . . . . . 90

5.3 .5 Seleção de um Modelo e Análise Final . . . . . . . . . . . . . . . . . . . . 91

5.4 Conclusões sobre o Capítulo . . . . . . . . . . . . . . . . . . . . . 97

6 Comentários e Perspectivas para Novas Pesquisas 99

A Condicionais Completas para o Modelo Kd3PP-tA 103

A.1 Resultados Úteis . . . . . . . . . . . . . . . . . . . . . . . 103

A.1.1 Soma de Formas Quadráticas . . . . . . . . . . . . . . . 103

A.1.2 Inversa da Soma de Matrizes . . . . . . . . . . . . . . . . . . . . 104

A.1.3 Posteriori do Coeficiente $\boldsymbol{\beta}$ de um Modelo de Regressão Linear Normal/Normal104

A.2 Condicional completa de $Q=\Sigma_{\theta}^{-1} \ldots \ldots \ldots$. . . . . . . . 106 
A.3 Condicional Completa de $\boldsymbol{\mu}_{\theta} \ldots \ldots \ldots$. . . . . . . . . . . . 106

A.4 Condicional Completa de $\left(U_{i j}, V_{i j}\right)^{\prime} \ldots \ldots \ldots$. . . . . . . . . . 108

A.5 Condicionais Completas de $\boldsymbol{Z}=\left(\boldsymbol{Z}_{1}, \ldots, \boldsymbol{Z}_{N}\right) \ldots \ldots$. . . . . . . 108

A.6 Condicional Completa de $\boldsymbol{\theta}=\left(\boldsymbol{\theta}_{1}^{\prime}, \ldots, \boldsymbol{\theta}_{N}^{\prime}\right) \ldots \ldots$. . . . . . . . . . 109

A.7 Condicional Completa de $\boldsymbol{\beta}_{12 j}=\left(\beta_{1 j}, \boldsymbol{\beta}_{2 j}^{\prime}\right)^{\prime} \ldots \ldots \ldots$. . . . . . . 110

A.8 Condicional Completa de $\beta_{3 j} \ldots \ldots \ldots$. . . . . . . . . . . 111

A.9 Condicional Completa de $\omega_{i}, \quad i=1, \ldots, N \ldots \ldots$. . . . . . . . . . 111

A.10 Condicional Completa de $\delta$ : Caso Unidimensional . . . . . . . . . . . . 112

B Função MCMCirtKd2PPSn para a estimação do modelo Kd2PPSn 114

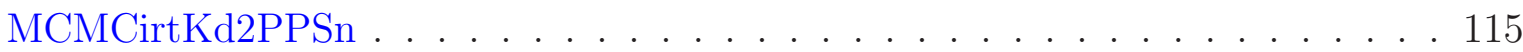




\section{Notações e Abreviações Utilizadas no Texto}

\section{Expressões / Abreviações}

e.g.: por exemplo (exempli gratia)

i.i.d. : independentes e identicamente distribuídas

MRI: Modelo de Resposta ao Item

FRI: Função de Resposta ao Item

CCI: Curva Característica do Item

EMV: Estimador de Máxima Verossimilhança

MVM: Máxima Verossimilhança Marginal

MVM-EM: Máxima Verossimilhança Marginal EM (Esperança e Maximização)

$K$ dlPP / $K$ mlPP: Modelo $K$-dimensional / $K$-multivariado de $l$ parâmetros probito ( $K \mathrm{~d} 1 \mathrm{PP}, K \mathrm{~d} 2 \mathrm{PP}$ e $K \mathrm{~d} 3 \mathrm{PP}, K$ sendo um número inteiro positivo)

$K$ dlPPN: Modelo $K$-dimensional de $l$ parâmetros probito com distribuição Normal para os traços latentes

( $K \mathrm{~d} 1 \mathrm{PPN}, K \mathrm{~d} 2 \mathrm{PPN}$ e $K \mathrm{~d} 3 \mathrm{PPN}, K$ sendo um número inteiro positivo)

$K$ dlPPSn: Modelo $K$-dimensional de $l$ parâmetros probito com distribuição "Skewnormal" (normal assimétrica) para os traços latentes

( $K$ d1PPSn, $K$ d2PPSn e $K$ d3PPSn, $K$ sendo um número inteiro positivo)

\section{Nomenclatura}

$N(0,1)$ : distribuição Normal padrão

$S T_{\nu}\left(\boldsymbol{\mu}_{\theta}, \boldsymbol{\Sigma}_{\theta}, \boldsymbol{D}\right)$ : distribuição multivariada t-assimétrica com parâmetros $\boldsymbol{\mu}_{\theta}, \boldsymbol{\Sigma}_{\theta}, \boldsymbol{D}$ e $\nu$ e $D$

$\operatorname{Sn}\left(\boldsymbol{\mu}_{\theta}, \boldsymbol{\Sigma}_{\theta}, \boldsymbol{D}\right)$ : distribuição multivariada normal assimétrica com parâmetros $\boldsymbol{\mu}_{\theta}, \boldsymbol{\Sigma}_{\theta}$ 


\section{Quantidades e Parâmetros}

$N$ : Número de indivíduos envolvidos na análise

$J$ : Número de itens envolvidos na análise

$K$ : Dimensão do vetor de traços latentes

$\boldsymbol{\beta}_{1}=\left(\boldsymbol{\beta}_{11}, \ldots, \boldsymbol{\beta}_{1 j}, \ldots, \boldsymbol{\beta}_{1 J}\right)^{\prime}$ : vetor $J$-dimensional de interceptos relacionados aos níveis de dificuldade de $J$ itens

$\boldsymbol{\beta}_{2}:$ matriz $J \times K \operatorname{com} \boldsymbol{\beta}_{2 j}^{\prime}=\left(\beta_{2 j 1}, \ldots, \beta_{2 j k}, \ldots, \beta_{2 j K}\right)$ sendo a $j$-ésima linha correspondente ao vetor de $K$ parâmetros de discriminação associados ao poder discriminatório do $j$-ésimo item multidimensional

$\boldsymbol{\beta}$ : conjunto integral de parâmetros de itens envolvidos na análise

$\boldsymbol{\theta}$ : matriz $N \times K$ cuja $i$-ésima linha corresponde ao traço latente (multidimensional) associado ao $i$-ésimo respondente, $\boldsymbol{\theta}_{i}^{\prime}=\left(\theta_{i 1}, \ldots, \theta_{i k}, \ldots, \theta_{i K}\right)$; e

$\eta_{i j}$ : parâmetro que depende dos parâmetros do item $i$ e respondente $j$

$\boldsymbol{\eta}$ : parâmetros populacionais relacionados à distribuição dos traços latentes

$\boldsymbol{\mu}_{\theta}$ : parâmetro de localização da distribuição dos traços latentes

$\Sigma_{\theta}$ : parâmetro de escala da distribuição dos traços latentes

$\boldsymbol{D}$ : matriz diagonal com elementos correspondentes aos parâmetros de assimetria $\delta_{1}, \ldots, \delta_{K}$ da distribuição dos traços latentes

$\nu$ : graus de liberdade da distribuição dos traços latentes

\section{Símbolos}

$\sim$ : tem distribuição

$\propto$ : proporcional

$\equiv$ : equivalente

Matos, G. S. 
Capítulo 1

\section{Introdução}

\subsection{Introdução}

A implementação e o desenvolvimento de estudos, pesquisas e avaliações sobre o Sistema Educacional Brasileiro é um assunto que tem despertado bastante interesse de gestores, pesquisadores, educadores e público em geral envolvido com atividades de ensinoaprendizagem. O Sistema de Avaliação da Educação Básica (Saeb), a Prova Brasil, o Projeto PDE/Fundescola e o Exame Nacional de Ensino Médio (Enem) coordenado pelo Instituto Nacional de Estudos e Pesquisas Educacionais Anísio Teixeira (Inep), do MEC, são exemplos de sistemas de avaliação educacional que refletem este interesse. Um importante sistema de avaliação educacional desenvolvido à nível estadual é o Sistema de Avaliação de Rendimento Escolar do Estado de São Paulo (SARESP) que desde 1996 avalia as habilidades cognitivas desenvolvidas pelos alunos durante o processo de escolarização em séries e componentes curriculares diversas (Bitar et al., 1998). Nas últimas versões, este sistema concentrou-se na avaliação das habilidades cognitivas de Leitura e Escrita e em 2005 a área de Matemática foi acrescida ao conjunto de testes.

Em todos os sistemas citados acima, assim como em outros, o uso de tecnologias avançadas que permitam a coleta e a análise de dados sob condições de avaliação cada vez mais complexas, têm sido fundamental para produzir informações mais confiáveis e de maior relevância para a análise e o diagnóstico de um sistema educacional. Diante desta realidade, a Teoria da Resposta ao Item (TRI), que surgiu na literatura psicométrica, tem desempenhado um papel de grande importância para a modelagem e a análise de dados provenientes de testes cognitivos (Lord; Novick, 1968; van der Linden; Hambleton, 1997). Devido a esta teoria, dificuldades antes encontradas pela Teoria Clássica de Testes (Lord; Novick, 1968) foram em grande parte superadas e novas informações tornaram-se possíveis de serem disponibilizadas. A comparação entre diferentes grupos de indivíduos sem a necessidades de se construir testes equivalentes; ou seja; testes sem o mesmo grau de dificuldade e a possibilidade de se interpretar escalas de medidas segundo características pedagógigas dos itens são apenas algumas das vantagens atribuídas à TRI (Andrade et al., 2000; Andrade, 2001). A TRI no Brasil, devido sua importância, além de estar sendo utilizada em sistemas de avaliação educacional tais como o Saeb, Prova Brasil e SARESP; 
também tem sido estudada e desenvolvida por diversos autores brasileiros que dentre outros podemos citar: Tavares (2001), Andrade et al. (2000), Andrade (2001), Matos (2001), Nojosa (2002), Azevedo (2003), Bortolotti (2003), Bazán (2005), Cúri (2006) e Azevedo (2008).

A Teoria da Resposta ao Item (TRI) é uma teoria que procura explicar as características dos itens que compõe um teste e de um ou mais grupos de respondentes por meio de probabilidades (modelos) de resposta aos itens. De modo geral, os itens que compõe um teste possuem características, tais como: nível de dificuldade, discriminação e/ou probabilidade de acerto casual que podem ser estimadas, analisadas e interpretadas segundo um determinado modelo de resposta ao item da TRI* (Andrade et al., 2000). Da mesma forma, a estimação e a análise de uma ou mais características não-observáveis dos respondentes que denominamos habilidade(s), constructo(s) ou, num contexto geral, traço(s) latente(s), também pode ser realizada através de um modelo de resposta ao item da TRI (van der Linden; Hambleton, 1997; Andrade et al., 2000).

Nos últimos anos, um dos modelos de resposta ao item que tem recebido bastante atenção é o K-dimensional de 2 Parâmetros Probito (Kd2PP) (Reckase, 1997b) o qual é adequado para a análise de testes em que cada item exige do respondente dois ou mais traços latentes para que uma resposta correta seja obtida (multidimensional dentro-doitem; Adams et al. (1997), te Marvelde et al. (2006)). Isto é o que ocorre, por exemplo, com os itens de um teste do Enem do Inep/MEC pois, em geral, para que um determinado item seja respondido corretamente é necessário que o aluno tenha conhecimentos de português, geografia, matemática e/ou outra disciplina. Sendo assim, cada item valoriza o conhecimento interdisciplinar e contextualizado e o teste é dito ser do tipo multidimensional (Nojosa, 2002). Um outro tipo de modelo de resposta ao item, também de grande importância, é o K-multivariado de 2 Parâmetros Probito (Km2PP) (multidimensional entre itens; Adams et al. (1997), te Marvelde et al. (2006)) o qual de fato pode ser visto como um sub-modelo do K-dimensional, fato este que facilita bastante o desenvolvimento de todo o processo de estimação pois uma vez desenvolvido o processo de estimação do modelo K-dimensional é necessário apenas fixar adequadamente alguns parâmetros de tal modelo para obter os resultados desejados e inerentes ao K-multivariado (veja Capítulo 3 deste trabalho e item (c) da sub-sub-seção 4.2.4.2). Este modelo, assim como o Kd2PP, também é adequado para a análise de um teste em que as respostas aos itens dependem de múltiplos traços latentes dos respondentes. Entretanto, a adequação deste modelo é para a análise de um teste que pode ser dividido em sub-conjuntos de itens ou sub-testes cujas respostas dependem de forma total ou preponderante de apenas um dos múltiplos traços latentes. Os testes relacionados ao Projeto PDE/Fundescola do INEP/MEC, Prova Brasil e SARESP são alguns exemplos em que o uso de um modelo Km2PP é mais ade-

* Se o modelo de resposta ao item contempla apenas o nível de dificuldade, dizemos que o modelo é de 1 parâmetro; se contempla o nível de dificuldade e discriminação, 2 parâmetros e se contempla o nível de dificuldade, discriminação e probabilidade de acerto casual, o modelo é dito ser de 3 parâmetros.

Matos, G. S.

IME/USP 
quado do que o Kd2PP pois nestes testes um mesmo grupo de alunos é submetido a um teste de Português e outro de Matemática de modo que conjuntamente constituem o que preferimos denominar teste do tipo multivariado (Matos, 2001).

\subsection{Objetivos e Organização da Tese}

A motivação do presente trabalho surge da necessidade contínua de novos estudos com relação à modelos e métodos de estimação na área da TRI e, em particular, sobre aqueles em que a análise de dois ou mais processos cognitivos ou traços latentes são de interesse, assim como acontece na análise de dados provenientes de testes do tipo multidimensional e multivariado. Especificamente, observamos que a distribuição normal padrão (uni ou multivariada) é a mais utilizada na modelagem e/ou no desenvolvimento dos métodos de estimação dos modelos de resposta ao item e dentre outras justificativas seu uso encontra apoio em resultados assintóticos que indicam pouca influência da distribuição (distribuição a priori, no contexto bayesiano) dos traços latentes sobre a distribuição marginal dos dados (distribuição a posteriori, no contexto bayesiano) na medida em que a quantidade de itens aumenta (Chang; Stout, 1993; Chang, 1996; Froelich, 2000).

Bock e Aitkin (1981), no contexto da inferência freqüentista, realizaram um estudo empírico considerando apenas distribuições (distribuição a priori, no contexto bayesiano) para os traços latentes do tipo simétrica; e.g. normal e uniforme; e também concluíram que a distribuição dos traços latentes exerce pouca influência sobre as estimativas de máxima verossimilhança marginal dos parâmetros dos itens. Entretanto, no estudo de Bock e Aitkin (1981) nenhum resultado foi apresentado sobre a influência de tais distribuições com relação às estimativas dos traços latentes ou qualquer outra característica da população de respondentes. Apesar destes estudos indicarem a pouca relevância da especificação de uma distribuição para os traços latentes sobre as estimativas dos itens, ainda podemos observar na literatura uma real necessidade de novas pesquisas nesta área, especialmente quando existe alguma evidência empírica de que a verdadeira distribuição é assimétrica. Como exemplo, podemos citar a situação em que respondentes a um determinado teste são de alguma forma pré-selecionados e um dos objetivos é estimar os vetores de traços latentes dos respondentes e/ou características populacionais tais como média e matriz de variâncias-covariâncias da distribuição dos traços latentes (Tavares (2001, Sec. 10.2 e 10.9), Matos (2001, Cap. 8) e Azevedo (2003, Sec. 10.2)).

Recentemente, a falta de alternativas ao modelo normal uni/multivariado já é um problema superado pois atualmente é possível encontrar inúmeros trabalhos que introduzem e desenvolvem generalizações da distribuição normal com relação à assimetria, curtose e/ou multimodalidade (Genton, 2004; Branco; Arellano-Valle, 2004; Arellano-Valle et al., 2006). No contexto dos modelos unidimensionais da TRI, por exemplo, Bazán (2005) percebeu esta realidade e introduziu uma classe denominada PANA (Probito Assimétrico -

Matos, G. S.

$\mathrm{IME} / \mathrm{USP}$ 
Normal Assimétrica) a qual permite modelar possíveis comportamentos assimétricos de um modelo (uma probabilidade) de resposta ao item bem como a especificação de uma distribuição normal assimétrica para os traços latentes (unidimensionais) a qual é utilizada no processo de estimação.

Motivados pela necessidade de melhor representar os fenômenos da área psicométrica ${ }^{\dagger}$ e da atual disponibilidade de distribuições elípticas assimétricas cujas propriedades são tão convenientes quanto aquelas devidas à distribuição normal, a proposta do presente trabalho é apresentar uma extensão do modelo K-dimensional de 3 Parâmetros Probito (Sub-seção 3.2.3 deste trabalho) com vetores de traços latentes normalmente distribuídos para o caso t-Assimétrico, gerando, assim, o que denominaremos modelo Kd3PP-tA. Nossa proposta, portanto, pode ser considerada como uma extensão do trabalho desenvolvido por Bazán (2005) tanto no sentido de extender a distribuição unidimensional assimétrica dos traços latentes para o caso multidimensional quanto no que conscerne em considerar o achatamento (curtose) da distribuição. Ressaltamos, entretanto, que esta extensão não é direta pois nossa proposta terá como base a classe de distribuições elípticas assimétrica multivariada desenvolvida por Sahu et al. (2003) e não a família de distribuições assimétricas de Azzalini (1985), Azzalini e Dalla Valle (1996) e Azzalini e Capitanio (1999). Nossa proposta também pode ser vista como uma extensão do trabalho de Béguin e Glas (2001) no sentido de desenvolver o método de estimação bayesiana dos modelos multidimensionais da TRI via DAGS (Dados Aumentados com Amostrador de Gibbs) para o caso em que os vetores de traços latentes comportam-se segundo uma distribuição multivariada t-Assimétrica.

Com o intuito de desenvolver a presente tese apresentamos no Capítulo 2 uma modelagem probabilística dos modelos multidimensionais da TRI sob um contexto bastante geral juntamente com um estudo bibliográfico que procura discutir sobre os principais métodos de estimação e inferência existentes na literatura da TRI. Nesta pesquisa bibliográfica, procuramos discutir sobre os principais problemas encontrados nos processos de estimação dos modelos da TRI e ao final procuramos justificar a escolha de um dos métodos investigados. Com relação a esta escolha, antecipamos que os métodos bayesianos via Monte Carlo por Cadeias de Markov (MCMC) são os mais promissores para a análise de dados que exigem o uso de modelos cada vez mais complexos e adequados à realidade.

No Capítulo 3, apresentamos e discutimos aspectos importantes de um modelo que denominamos K-dimensional de 2 Parâmetros Probito (Kd2PP) o qual pertence à classe geral dos modelos multidimensionais desenvolvida e discutida no Capítulo 2. Neste capítulo, também discutimos sobre uma das principais dificuldades encontradas no processo de estimação e inferência dos modelos multidimensionais da TRI que é a falta de identi-

$\dagger$ Heinen (1996, p. 105), por exemplo, ressaltou que a especificação da distribuição normal para as variáveis latentes em uma análise de dados de atitude, em geral, não é uma escolha muita adequada para descrever a distribuição populacional.

Matos, G. S.

IME/USP 
ficabilidade. A discussão sobre este tema foi desenvolvida tanto no contexto da inferência clássica quanto bayesiana, de modo que pudéssemos ampliar e desmistificar nossos conhecimentos sobre um assunto ainda pouco discutido na literatura estatística. Tal discussão pretende, portanto, ser uma das principais contribuições do presente trabalho.

No Capítulo 4, o método de estimação bayesiana do modelo Kd3PP da TRI via DAGS, proposto por Béguin e Glas (2001), é extendido para o caso em que os vetores de traços latentes comportam-se segundo uma distribuição multivariada t-Assimétrica. Ainda neste capítulo, procuramos ressaltar a importância de um ambiente computacional eficiente e flexível para a implementação de algoritmos que requerem um desempenho cada vez mais elevado dos computadores. Diante desta preocupação nós propomos o uso do ambiente computacional, gráfico e estatístico $R$ o qual pode ser integrado à funções implementadas na linguagem $\mathrm{C}++$, viabilizando assim o processamento de algoritmos de forma mais rápida e eficiente, além de permitir a realização de análises mais ricas dos dados disponibilizados pela estimação bayesiana de parâmetros via métodos de Monte Carlo por Cadeias de Markov (MCMC).

Devido a existência de várias tipos de itens e testes, além de várias possibilidades de modelagem das respostas aos itens e de distribuições a serem consideradas para os traços latentes no processo de estimação, apresentamos no Capítulo 5 análises de apenas alguns conjuntos de dados simulados cujo intuito é identificar situações particulares em que o uso de uma distribuição normal assimétrica para os traços latentes é de maior relevância para a estimação e inferência dos parâmetros de item bem como outros parâmetros relacionados à distribuição dos traços latentes. Para finalizar, aspectos importantes sobre o presente trabalho e perspectivas para novas pesquisas são apresentados no Capítulo 6. Alguns desenvolvimentos teóricos são decritos no Apêndice A seguido pelo Apêndice $\mathrm{B}$ onde apresentamos informações sobre como um usuário $\mathrm{R}$ pode ter acesso à função MCMCirtKd2PPSn desenvolvida para a realização das análise do Capítulo 5.

Matos, G. S.

$\mathrm{IME} / \mathrm{USP}$ 
Capítulo 2

\section{Modelos Multidimensionais da TRI}

Neste capítulo, o principal objetivo será apresentar uma base teórica para o desenvolvimento de modelos multidimensionais da TRI. Com este intuito, uma estrutura probabilística geral é desenvolvida na Seção 2.1, onde são apresentadas expressões, suposições e aspectos importantes para uma análise de dados por meio da TRI. Na Seção 2.2, os principais métodos de estimação encontrados na literatura da TRI são discutidos. A importância desta seção deve-se a necessidade de se escolher e/ou desenvolver novos métodos de estimação e inferência para os parâmetros de um modelo qualquer e, em particular, para os que serão desenvolvidos neste trabalho. Com relação ao método de estimação e inferência escolhido, uma breve justificativa é apresentada na última seção deste capítulo (Seção 2.3).

\subsection{Modelos Multidimensionais da TRI}

Inicialmente, considere $\boldsymbol{Y}_{i}$. um vetor aleatório cujas componentes $Y_{i j}$ representam a resposta, que em geral é discreta, do $i$-ésimo respondente para o $j$-ésimo item de um teste qualquer. Considere, ainda, o modelo probabilístico

$$
p_{i j}=F_{j}\left(\eta_{i j}\right)=P\left(Y_{i j}=y_{i j} \mid \boldsymbol{\theta}_{i}, \boldsymbol{\beta}_{j}\right),
$$

$i=1, \ldots, N, j=1, \ldots, J$, para representar a probabilidade (condicional) de uma determinada resposta $y_{i j}$, dado um vetor $K$-dimensional de traços latentes $\boldsymbol{\theta}_{i}=\left(\theta_{i 1}, \ldots, \theta_{i k}, \ldots, \theta_{i K}\right)^{\prime}$ e um vetor de parâmetros correspondentes às características do item $j, \boldsymbol{\beta}_{j}$. A quantidade $\eta_{i j} \in \mathbb{R}$ é uma função adequadamente escolhida dos parâmetros $\boldsymbol{\theta}_{i} \in \mathbb{R}^{K}$ e $\boldsymbol{\beta}_{j} \in \mathbb{R}^{l+K}$ com $l=1$ ou $l=2$ com possíveis restrições no espaço paramétrico de $\boldsymbol{\beta}_{j}$ que dependerá da FRI considerada, conforme veremos neste trabalho.

Na TRI, o modelo probabilístico (2.1) é comumente denominado Curva Característica do Item (CCI), Modelo de Resposta ao Item (MRI) ou Função de Resposta ao Item (FRI). Na área educacional, por exemplo, FRI's baseadas em funções de distribuição acumulada expressam a idéia de que a probabilidade de resposta correta a um item (ou a obtenção de um escore mais alto) aumenta na medida em que cada componente do vetor 
de traços latentes que, neste caso, representam habilidades, proficiências, competências ou capacidades sobre determinadas áreas do conhecimento humano, também aumenta. Em van der Linden e Hambleton (1997), Adams et al. (1997) e Andrade et al. (2000), por exemplo, vários destes modelos podem ser encontrados. Por outro lado, FRI's do tipo nãoacumulativas são mais adequadas para aplicações cujo maior interesse é o comportamento e/ou a opinião de indivíduos. Estes são mais conhecidos por modelos "unfolding" ou de desdobramentos e podem ser vistos em Roberts et al. (2000) e Bortolotti e Andrade (2007). Recentemente, modelos de resposta ao item assimétricos e relacionados à situações constrangedoras também têm sido propostos na literatura da TRI (Bazán et al. (2006), Cúri (2006), respectivamente).

No presente capítulo, devemos destacar que a expressão (2.1) representa o início da construção de uma classe bastante ampla para a análise de respostas aos itens. Note, por exemplo, que o uso do índice $j$ na FRI, $F$, leva em consideração a possibilidade de se modelar um teste (conjunto de itens) do tipo misto; ou seja; um teste que pode ser composto por diferentes tipos de itens com relação à escala de mensuração, podendo ser: dicotômicos, nominais, ordinais ou contínuos e admitindo ou não a presença de múltiplos avaliadores (ver, e.g., van der Linden e Hambleton (1997), Patz e Junker (1999b) e Baker e Kim (2004)). Expressar o modelo (2.1) como função de um vetor de traços latentes, $\boldsymbol{\theta}_{i}$, também possibilita a modelagem de testes não somente com relação aos tipos e características dos itens mas também com relação à dimensionalidade de $\boldsymbol{\theta}_{i}$; podendo ser multidimensional ou unidimensional conforme o número de traços latentes exigidos para se responder a um determinado item ou conjunto dos mesmos (van der Linden; Hambleton, 1997; Fischer; Molenaar, 1995).

Continuando a caracterização dos modelos da classe multidimensional da TRI consideraremos que:

- $\boldsymbol{Y}_{i .}=\left(\boldsymbol{Y}_{i 1}, \boldsymbol{Y}_{i 2}, \ldots, \boldsymbol{Y}_{i J}\right)^{\prime}$ denotará o vetor total de respostas do respondente $i$ submetido a um determinado teste composto por $J$ itens.

- $\boldsymbol{Y}=\left(\boldsymbol{Y}_{1 .}^{\prime}, \ldots, \boldsymbol{Y}_{i .}^{\prime}, \ldots, \boldsymbol{Y}_{N}^{\prime}\right)^{\prime}$ denotará a matriz $N \times J$ das respostas associadas a todos os $N$ respondentes cuja $i$-ésima linha é $\boldsymbol{Y}_{i \text {. }}^{\prime}=\left(Y_{i 1}, \ldots, Y_{i j}, \ldots, Y_{i J}\right)$.

\subsubsection{Sobre a Ausência de Dados}

Ao introduzir a notação na seção anterior é interessante observar que admitimos a aplicação de exatamente $J$ itens a cada um dos respondentes. Entretanto, devido à propriedade de Invariância da TRI a qual diz que as estimativas dos traços latentes (das características dos itens) são invariantes ao conjunto de itens (de respondentes) a que são submetidos; a avaliação de um constructo (traço latente) pode ser realizada através da aplicação de um conjunto menor de itens a cada um dos respondentes e isto pode ser feito sem

Matos, G. S.

$\mathrm{IME} / \mathrm{USP}$ 
deixar de aplicar o conjunto total de itens ao total de respondentes (Hambleton et al., 1991; Kolen; Brennan, 1995; Andrade et al., 2000). Um procedimento como este, conforme Tavares (2001, Cap. 6) se referiu, produz a Ausência de Resposta por Planejamento e conforme descrito por Holman e Glas (2005) e discutido por Mislevy e Wu (1996) e Mislevy e Chang (2000), estes tipos de dados ausentes devem ser considerados como perdidos completamente ao acaso ("Missing Completely at Random"-MCAR; Rubin (1976), Little e Rubin (1987)). Neste caso, a modelagem dos dados fica consideravelmente simplificada pois não há a necessidade de se incorporar ao modelo dos dados observáveis um outro modelo (probabilístico) associado ao mecanismo de perda de dados. Apesar disto, é importante considerarmos a necessidade de se introduzir ao modelo, ou melhor, ao conjunto de dados observáveis, um conjunto de variáveis indicadoras, - aleatórias mas com distribuição ignorável-, que denotaremos por uma matriz

$$
\boldsymbol{M}=\left[m_{i j}\right]
$$

de dimensão $N \times J$ com linhas $m_{i .}^{\prime}=\left(m_{i 1}, \ldots, m_{i j}, \ldots, m_{i J}\right)$ em que $m_{i j}, i=1, \ldots, N, j=$ $1, \ldots, J$, assume o valor 1 ou 0 , conforme o item $j$ de um teste composto por $J$ itens seja submetido ao $i$-ésimo respondente ou não, respectivamente.

Outro tipo de resposta ausente ocorre quando um item é submetido a um determinado respondente e o mesmo não o responde. Neste caso, considerar o menor escore admissível ao item (incorreto se item do tipo dicotômico) ou simplesmente ignorar a ausência de tal resposta, assim como descrito no parágrafo anterior, são algumas das opções a serem adotadas. Agora, é importante destacar que a adoção de tais procedimentos para este tipo específico de dados ausentes pode levar à estimativas viesadas para os parâmetros dos itens, $\boldsymbol{\beta}$, pois o mecanismo de perda de dados poderá estar associado às características do $i$-ésimo respondente, $\boldsymbol{\theta}_{i}$, e/ou do $j$-ésimo item, $\boldsymbol{\beta}_{j}$. Sendo assim, práticas mais cautelosas podem ser adotadas tal como Holman e Glas (2005) propuseram. Estes autores, apresentaram 4 modelos bastante gerais como possibilidades para a modelagem de dados (respostas) observáveis e não-observáveis (veja também, Patz (1996, Sec. 4.3)). O uso de tais modelos, possibilitam, por exemplo, avaliar o grau de ignorabilidade das respostas ausentes (Holman; Glas, 2005) e/ou a previsão de respostas aos itens não respondidos (Patz; Junker, 1999b).

Na próxima sub-seção descreveremos as suposições necessárias à construção da verossimilhança associada aos modelos multidimensionais da TRI.

\subsubsection{Suposições}

Na construção de modelos probabilísticos é bastante comum adotar suposições que simplifiquem o desenvolvimento teórico do processo de estimação dos parâmetros. Duas delas, bastante conhecidas no contexto da TRI, exigem que:

Matos, G. S.

IME/USP 
(S1) Respostas provenientes de indivíduos distintos são independentes desde que seja conhecido o verdadeiro nível (valor) do vetor de traços latentes (habilidades), $\boldsymbol{\theta}_{i}$, do indivíduo $i, i=1, \ldots, N$.

(S2) Respostas aos itens de um teste são independentes desde que seja conhecido o verdadeiro nível (valor) do vetor de traços latentes (habilidades), $\boldsymbol{\theta}_{i}$, do indivíduo $i$.

No contexto da suposição (S1) é importante destacar que em algumas situações, tais como em grandes avaliações educacionais, grupos de alunos (respondentes) pertencem a uma mesma sala de aula a qual sofre influência das características de uma mesma escola, cidade, região, etc. Sendo assim, é possível que a suposição (S1) deixe de ser razoável e, neste caso, a recomendação é trabalhar com os modelos do tipo hierárquico/multinível (Rijmen et al., 2003; Fox, 2005; Azevedo, 2008). Também é importante destacar que a suposição (S2) é mais conhecida por Independência Condicional (ou Local) e que o estudo sobre sua validade têm recebido atenção especial por parte de alguns autores. De fato, a hipótese de dimensionalidade de um teste é mais importante do que a de independência local pois a dimensionalidade $K$ de um teste tem por definição tradicional a quantidade (dimensão) máxima de traços latentes necessária para produzir um modelo de variáveis latentes (e.g. da TRI) localmente independente e monótono (Stout, 1990). Neste sentido, é de fundamental importância que parte da análise de um conjunto de itens via modelos da TRI seja dedicada a análise e/ou verificação da dimensionalidade dos itens e testes (Nojosa (2002), Froelich (2000) e Stout (1990)), pois, como podemos ver na literatura, a correta especificação da dimensionalidade do traço latente e conseqüente independência condicional constitui uma das suposições básicas da maioria destes modelos (detalhes em Hambleton et al. (1991), Lord (1980), Lord e Novick (1968)). No presente trabalho, consideraremos as questões discutidas acima como sendo resolvidas e, portanto, com as suposições $(\mathbf{S 1})$ e $(\mathbf{S 2})$ sendo válidas.

Outra observação a ser destacada consiste em dizer que num primeiro momento será razoável alguém pôr em dúvida a suposição (S2) uma vez que não é esperado que as respostas provenientes de um mesmo indivíduo sejam independentes. Entretanto, não é isto que tal suposição expressa mas sim que as respostas aos itens são independentes sob a hipótese de conhecermos o verdadeiro valor dos traços latentes. Em outras palavras, isto quer dizer que a informação sobre o verdadeiro valor do vetor de traços latentes de um respondente é suficiente para determinarmos a probabilidade de um indivíduo responder corretamente a um item (fixados os parâmetros dos itens). Pode-se dizer ainda que o valor do vetor de traços de um indivíduo permanece inalterado após responder um item após o outro, ou seja, o indivíduo não adquire conhecimento algum sobre o que está sendo avaliado ao longo de um teste. Desta maneira, o valor do traço latente ao término de um teste é o mesmo do início.

Matos, G. S.

$\mathrm{IME} / \mathrm{USP}$ 


\subsubsection{Função de Verossimilhança}

Uma vez definidas as Funções de Resposta aos Itens em 2.1, notações e suposições, passamos a construção da função de verossimilhança sob a forma geral dos modelos multimensionais.

Usando a suposição (S2) temos que a probabilidade (condicional) de ocorrência de um determinado vetor de respostas associado ao $i$-ésimo respondente submetido a um teste é dada por:

$$
P\left(\boldsymbol{Y}_{i .} \mid \boldsymbol{\theta}_{i}, \boldsymbol{\beta}\right)=\prod_{j \in \boldsymbol{J}}\left\{P\left(Y_{i j}=y_{i j} \mid \boldsymbol{\theta}_{i}, \boldsymbol{\beta}_{j}\right)\right\}^{m_{i j}},
$$

onde $\boldsymbol{J}$ é o conjunto dos índices dos itens que compoem o teste, $\boldsymbol{\beta}=\left(\boldsymbol{\beta}_{1}^{\prime}, \ldots, \boldsymbol{\beta}_{J}^{\prime}\right)^{\prime}$ e as demais "quantidades" são tais como descritas nas seções anteriores (e.g., $m_{i j}$ encontra-se definido em 2.2).

Agora, a partir da suposição (S1) podemos obter o seguinte modelo probabilístico (condicional) para o total de respostas

$$
P(\boldsymbol{Y} \mid \boldsymbol{\theta}, \boldsymbol{\beta})=\prod_{i=1}^{N} P\left(\boldsymbol{Y}_{i .} \mid \boldsymbol{\theta}_{i}, \boldsymbol{\beta}\right),
$$

em que $\boldsymbol{\theta}$ é uma matriz $N \times K$ cuja $i$-ésima linha corresponde ao traço latente (multidimensional) associado ao $i$-ésimo respondente, $\boldsymbol{\theta}_{i}^{\prime}=\left(\theta_{i 1}, \ldots, \theta_{i k}, \ldots, \theta_{i K}\right)$.

Após obter um modelo tal como o expresso por (2.4), o procedimento usual é obter estimativas de máxima verossimilhança $\widehat{\boldsymbol{\theta}}$ e $\widehat{\boldsymbol{\beta}}$ a partir da maximização da função $\boldsymbol{d} \boldsymbol{e}$ verossimilhança dada por

$$
L(\boldsymbol{\theta}, \boldsymbol{\beta} \mid \boldsymbol{y}, \boldsymbol{M}) \equiv P(\boldsymbol{Y} \mid \boldsymbol{\theta}, \boldsymbol{\beta}) .
$$

Entretanto, este procedimento não deve ser feito de forma imediata, conforme veremos na próxima seção.

\subsection{Métodos de Inferência}

Na seção anterior apresentamos num contexto bastante geral uma maneira de se construir os modelos multidimensionais da TRI. Nesta seção, também num contexto geral, procuramos discutir sobre alguns problemas inerentes à estimação conjunta do vetor de parâmetros dos itens, $\boldsymbol{\beta}$, e dos vetores de traços latentes, $\boldsymbol{\theta}_{i}, i=1, \ldots, N$. Esses problemas serão inicialmente discutidos na próxima sub-seção sob a abordagem da inferência clássica por máxima verossimilhança e na sub-seção subseqüente apresentamos

Matos, G. S.

IME/USP 
algumas vantagens e desvantagens dos métodos de estimação sob o enfoque freqüentista e bayesiano. Finalmente, deixamos para a última seção deste capítulo um breve resumo de nossa decisão quanto ao método de estimação e inferência a ser utilizado na continuação deste trabalho. Devemos lembrar que situações em que o interesse é estimar apenas os parâmetros dos itens (traços latentes) com os traços latentes (itens) já conhecidos também ocorrem na prática, porém; para estes casos; o processo de estimação não apresenta grandes dificuldades (veja, por exemplo, Andrade et al. (2000) e Tavares (2001)).

\subsubsection{Estimação por Máxima Verossimilhança - MV}

Um dos problemas freqüentemente encontrado na TRI ocorre quando existe o interesse em realizar a estimação conjunta do vetor de parâmetros dos itens, $\boldsymbol{\beta}$, e dos vetores de traços latentes, $\boldsymbol{\theta}_{i}, i=1, \ldots, N$, através do método de máxima verossimilhança. $\mathrm{O}$ problema fica caracterizado quando valores distintos dos parâmetros implicam em um mesmo valor para o modelo probabilístico, inviabilizando, portanto, o desenvolvimento de qualquer procedimento de otimização direta da função de verossimilhança. Na literatura da TRI este problema é mais conhecido por indeterminação da escala de medida ou métrica quando rigorosamente deveria ser conhecido como um problema de falta de identificabilidade do modelo pois o que realmente acontece é que a solução para a falta de identificabilidade de um modelo da TRI* induz ao estabelecimento de uma escala de medida ou métrica para os valores dos parâmetros dos itens e/ou traços latentes que inicialmente é arbitrária. Neste sentido, o problema da falta de identificabilidade antecede o da indeterminação da escala de medida de modo que uma nova escala pode ser determinada após o problema da falta de identificabilidade ter sido resolvido e estimativas obtidas Por exemplo, através de transformações lineares (Andrade et al., 2000). Veja também exemplos desenvolvidos no Capítulo 5 do presente trabalho.

A solução para a falta de identificabilidade na TRI depende basicamente do modelo em estudo e do método de estimação adotado. Nos modelos unidimensionais, por exemplo, quando o método de Máxima Verossimilhança Conjunta (estimação por MV dos itens e traços latentes) é adotado, essa arbitrariedade é eliminada introduzindo-se no processo de estimação alguma restrição (normalização) sobre os parâmetros dos itens ou traços latentes (veja, por exemplo, Baker e Kim (2004, p. 96 e 142) e Andrade et al. (2000, p. 48)). Por outro lado, quando o processo de estimação basea-se na distribuição marginal dos dados, tal como, Máxima Verossimilhança Marginal (Bock; Lieberman, 1970, MVM), MVM-EM (Bock; Aitkin, 1981) ou Estimação Bayesiana Marginal (Mislevy, 1986, EBM); uma solução natural é obtida ao fixar os parâmetros de localização e escala de uma distribuição escolhida para os traços latentes ${ }^{\dagger}$ (ver detalhes em Baker e Kim (2004, p. 174) e

* Este é um problema freqüentemente encontrado numa classe maior de modelos denominada modelos de variáveis latentes (Bartholomew, 1999).

Matos, G. S.

$\mathrm{IME} / \mathrm{USP}$ 
Andrade et al. (2000, p. 51 e 95)). Com relação à alguns modelos mais complexos encontrados na literatura da TRI; tais como os multidimensionais/multivariados e longitudinais; percebemos que as soluções propostas são simples extensões das discutidas acima (veja Bock e Zimowski (1997), Tavares (2001, Cap. 4) e Matos (2001, p. 36)). Agora, é importantes ressaltar que dependendo da estrutura paramétrica dos modelos multidimensionais, tais soluções podem constituir condições necessárias porém não suficientes, de modo que restrições adicionais sobre os parâmetros dos itens tornam-se necessárias (Rivers, 2003; Jackman, 2001). Por simplicidade de exposição, uma discussão detalhada sobre a falta de identificabilidade em alguns modelos multidimensionais da TRI é deixada para o Capítulo 3 (Secão 3.3).

Um outro problema freqüentemente encontrado na TRI, refere-se à inconsistência dos estimadores de máxima verossimilhança (EMV). Este problema pode ser percebido como uma consequência da superparametrização do modelo ou pela falta de identificabilidade. Com relação à superparametrização note que uma vez decidido (fixado) sobre quais instrumentos de medida (testes) serão aplicados a um grupo de respondentes, a quantidade de traços latentes $\boldsymbol{\theta}_{i}$ aumenta (ou diminui) de acordo com o número de indivíduos. Neste caso, os traços latentes são denominados parâmetros incidentais enquanto que os parâmetros associados aos itens são denominados estruturais. Estas denominações são devidas a Neyman e Scott (1948) que num contexto diferente ao da TRI, observaram que o EMV dos parâmetros estruturais podem ser inconsistentes (veja também Little e Rubin (1983)). Vários estudos foram desenvolvidos para resolver este problema e uma possível solução que não ocorre na prática, consiste em aumentar o número de itens e indivíduos simultaneamente numa mesma proporção (Lord, 1968; Lord, 1975; Swaminathan; Gifford, 1983). Ainda neste contexto, um estudo desenvolvido por Haberman (1977) mostrou que os estimadores de máxima verossimilhança são conjuntamente consistentes, porém sua demonstração ficou restrita ao modelo logístico de 1 parâmetro (ML1; Rasch (1960/1980)). Por outro lado, Douglas (1997) apresentou um resultado mais geral quando admitiu suposições menos restritivas quanto a forma funcional do modelo de resposta ao item. Para aqueles que desejarem realizar um levantamento histórico e bibliográfico sobre os problemas provocados pela presença de parâmetros incidentais nos modelos probabilísticos, recomendamos a leitura de Lancaster (2000). No que se refere à falta de identificabilidade (Rivers, 2003) lembra que identificabilidade; apesar de não ser suficiente; é uma condição necessária para a existência de estimadores consistentes.

Um procedimento bastante utilizado para eliminar problemas assintóticos (viés, inconsistência e ineficiência) dos EMV dos parâmetros estruturais (itens, no nosso caso) na presença de parâmetros incidentais (traços latentes), consiste em eliminar da verossimilhança a presença destes últimos ${ }^{\ddagger}$. Neste caso, os parâmetros incidentais são vistos como

$\dagger$ Neste contexto, Bartholomew (1999) mostra que os modelos (marginais) da TRI pertencem à classe linear geral de variáveis latentes.

Matos, G. S.

$\mathrm{IME} / \mathrm{USP}$ 
parâmetros de incômodo ou de pertubação, tendo em vista a falta de interesse direto por eles. Este procedimento na TRI tem grande aceitabilidade porque em muitos casos o principal objetivo é estimar os parâmetros dos itens e, possivelmente, de alguns ou de todos os elementos de um vetor de parâmetros populacionais, $\boldsymbol{\eta}$, associado a uma arbitrária distribuição de probabilidade atribuída aos traços latentes, $\Pi(\boldsymbol{\theta} \mid \boldsymbol{\eta})$. Este é o caso, por exemplo, do modelo para grupos múltiplos de Bock e Zimowski (1997), dados longitudinais com traços latentes univariados (Tavares, 2001) e traços latentes multivariados (Matos, 2001). Aliás, em todos estes modelos observou-se de modo empírico (simulação) que o problema de inconsistência dos EMV dos parâmetros dos itens é contornado pelo uso do método de Máxima Verossimilhança Marginal - EM ${ }^{\S}$. Uma prova analítica sobre a consistência e a normalidade assintótica dos estimadores de máxima verossimilhança dos itens pode ser vista em Froelich (2000, Cap. 5), porém sua demonstração é restrita ao caso em que o limite é tomado para o infinito não somente da quantidade de indivíduos mas também de itens. Além disso, sua demostração limitou-se apenas ao método de MVM inicialmente proposto para a TRI por Bock e Lieberman (1970). A "escassez" de provas analíticas nesta área, mostra, de certo modo, o quanto é difícil o desenvolvimento da Teoria Assintótica na TRI.

Outro método que estima os parâmetros dos itens sem a presença dos traços latentes é o de Máxima Verossimilhança Condicional (MVC) cuja aplicação é mais comum na classe dos modelos Rasch (Rasch, 1960/1980, Modelo Logístico de 1 Parâmetro). A tradição do uso deste método nesta classe deve-se ao fato da mesma pertencer à família exponencial de modo que estatísticas suficientes existem para os traços latentes (Andersen, 1980, Cap. $6)$.

\subsubsection{Inferência por MVM-EM, Bayesiana-EM e Bayesiana via Métodos MCMC}

A estimação por $\boldsymbol{M} \boldsymbol{V} \boldsymbol{M}-\boldsymbol{E} \boldsymbol{M}$ de Bock e Aitkin (1981) é certamente um dos métodos mais adotados na literatura da TRI e isto ocorre especialmente quando o modelo de resposta ao item não pertence à família exponencial (Baker e Kim (2004), van der Linden e Hambleton (1997), Andrade et al. (2000), Tavares (2001) e Azevedo (2003)). Seu uso possibilitou, por exemplo, a análise de testes com mais de 12 itens pois a estimação dos parâmetros dos itens é feita sem a presença dos traços latentes e a adoção de suposições adicionais sobre

$\ddagger$ Num contexto geral, as funções geradas por este procedimento são denominadas pseudoverossimilhanças e freqüentemente são mais simples do que as verossimilhanças genuínas (Pace; Salvan, 1997).

$\S$ Quando o modelo probabilístico não pertence à família exponencial, o algoritmo EM seria melhor denominado pseudo-EM ou do tipo EM pois, segundo Bock e Aitkin (1981, p. 448), existe um erro na prova de Dempster et al. (1977) (veja também Azevedo (2003, p. 19)). Flury e Zoppé (2000) chamam a atenção para o perigo de se chegar à conclusões errôneas com o uso dos algoritmos pseudo-EM.

Matos, G. S.

$\mathrm{IME} / \mathrm{USP}$ 
a matriz de derivadas segunda da log-verossimilhança marginal (ortogonalidade entre os parâmetros de itens distintos) reduziu de forma considerável o esforço computacional necessário à inversão de matrizes no processo de estimação (Andrade et al., 2000, p. 61). A obtenção de estimadores consistentes para os parâmetros dos itens através da verossimilhança marginal é outra característica que torna este método atraente (Cordeiro (1992); Froelich (2000, Cap. 5)). A Estimação Bayesiana Marginal (Mislevy, 1986, EBM que é uma adaptação direta do método de MVM-EM para a inferência bayesiana também tem sido bastante utilizada na prática (Mislevy; Bock, 1985).

Se por um lado o método de MVM-EM é freqüentemente adotado para a estimação dos modelos da TRI, observamos que a estimação bayesiana também é freqüentemente sugerida para a resolução de problemas que surgem no processo de maximização. Sabese, por exemplo, que em análises de testes educacionais, as estimativas divergem quando ocorrem padrões de respostas extremos e/ou aberrantes, ou seja, quando ocorrem escores nulos ou perfeitos para os itens e/ou indivíduos ou quando respostas corretas(incorretas) aos itens difíceis(fáceis) correspondem a indivíduos com baixa(alta) habilidade. Por outro lado, conforme observado por Andrade et al. (2000, Sec. 3.7), isto não ocorre na estimação bayesiana. Tavares (2001, Sec. 10.6), após desenvolver um modelo univariado da TRI para a análise de dados longitudinais e implementar o processo de estimação por MVMEM, também sugeriu o uso de métodos bayesianos para a estimação dos parâmetros. Tal sugestão foi motivada pela ocorrência observada de divergência ou obtenção de estimativas com valores fora de um intervalo admissível. Tavares (2001) observou, ainda, que tal instabilidade no processo iterativo computacional do método de MVM-EM é provocada principalmente por alguns parâmetros da distribuição dos traços latentes, em especial, quando estes assumem valores nas fronteiras dos respectivos espaços paramétricos.

Outro fato notável na literatura da TRI corresponde ao crescente uso de Cadeias de Markov Monte Carlo (MCMC) como uma forma de estimar os parâmetros de modelos bayesianos. Albert (1992), por exemplo, mostrou que o método de Dados Aumentados com Amostrador Gibbs (DAGS) é bastante eficaz para obter a distribuição a posteriori conjunta dos parâmetros dos itens e traços latentes. O uso de métodos MCMC mais gerais, tal como Metropolis-Hastings dentro do Amostrador Gibbs (MHDAG), proposto inicialmente na TRI por Patz e Junker (1999a) e Patz e Junker (1999b) também reforçam esta tendência. Neste contexto, estudos comparativos entre os métodos de MVM e MCMC indicam que MVM é "claramente" superior quando o critério é a acurácia das estimativas dos itens. Vale ressaltar, entretanto, que tal resultado foi observado com relação a modelos mais simples como; por exemplo; os de 2 parâmetros (Baker (1998, MVM $\times$ MCMC); Kim e Cohen (1999, April, EBM × MCMC)). Em um modelo mais complexo tal como o de resposta nominal de Bock (1972) que possui muitos parâmetros, Wollack et al. (2002) mostram que tanto o método de MVM quanto MCMC produz boas estimativas com níveis de acurácia praticamente idênticos e, por isso, eles consideram os métodos MCMC como uma boa alternativa ao de MVM, especialmente quando não há um algoritmo de MVM

Matos, G. S.

$\mathrm{IME} / \mathrm{USP}$ 
desenvolvido. Algumas referências adicionais que se utilizam dos métodos MCMC para a estimação de modelos cada vez mais complexos na área da TRI são: Béguin e Glas (2001), Bazán (2005), Fox (2005) e Azevedo (2008).

Ainda com relação ao método de MVM-EM, autores como te Marvelde et al. (2006) chamam a atenção para uma forte limitação do mesmo devido aos cálculos de integrais múltiplas de grandes dimensões via pontos de quadratura. Patz e Junker (1999a) e Patz e Junker (1999b) também chamam a atenção para o fato de que a implementação do método de MVM-EM fica cada vez mais difícil e inviável na medida que surgem modelos mais complexos (e.g. modelos para testes do tipo misto Patz e Junker (1999b), multidimensionais com um grande número de dimensões (te Marvelde et al., 2006), multinível (Fox, 2005) e longitudinal (Azevedo, 2008)). Além disso, o método de MVM-EM estima os itens com os traços latentes previamente fixados (e vice-versa) o que torna difícil incorporar a incerteza (erro-padrão) das estimativas dos itens no cálculo da incerteza (erro-padrão) inerente à inferência dos traços latentes. Alguns estudos indicam, por exemplo, que esta prática utilizada tanto na inferência clássica pelos métodos de máxima verossimilhança quanto bayesiana via bayes empírico pode sub-estimar seriamente a incerteza dos traços latentes, especialmente quando o tamanho da amostra (de respondentes) é moderado, e.g., N=400 (Tsutakawa; Johnson, 1990). De outra forma, implementar a estimação dos parâmetros de um modelo mais complexo utilizando um método MCMC sob o enfoque bayesiano é relativamente mais simples porque, diferentemente do que ocorre, por exemplo, em métodos que utilizam o algoritmo EM (Bock e Aitkin (1981, MVMEM); Mislevy (1986, EBM)), aproximações por ponto de quadratura (para o passo E) e o cálculo de derivadas (para o passo M) não são necessários. Outra vantagem devido à metodologia bayesiana é a facilidade com que a incerteza das estimativas dos itens são facilmentes incorporadas nas inferências dos traços latentes (e vice-versa). Além disso, a Inferência Bayesiana via métodos MCMC tem como produto final uma aproximação empírica das verdadeiras distribuições (a posteriori) dos parâmetros e não simplesmente estimativas pontuais como é o caso dos métodos de MVM-EM e EBM via algoritmo EM (Mislevy; Bock, 1985)).

Um importante trabalho para a Inferência Bayesiana dos modelos da TRI via MCMC deve-se a Albert (1992) que introduziu um esquema de Dados Aumentados com Amostrador Gibbs (DAGS) para o ajuste do modelo da ogiva normal de 2 parâmetros. Béguin e Glas (2001); por exemplo; além de generalizarem o trabalho de Albert (1992) para o caso do modelo da ogiva normal de 3 parâmetros (3PNO); também consideraram a análise bayesiana completa via DAGS de uma versão multidimensional do modelo 3PNO, bem como a análise desses modelos sob a existência de grupos múltiplos e desenhos experimentais incompletos. Sahu (2002), também extendeu o método de Albert (1992) para o caso do modelo (unidimensional) da ogiva normal de 3 parâmetros, porém o esquema de dados aumentados (variáveis auxiliares) utilizado para estimar o parâmetro de "acerto casual" foi ligeiramente distinto do proposto por Béguin e Glas (2001). Além disso, Sahu (2002)

Matos, G. S.

$\mathrm{IME} / \mathrm{USP}$ 
propôs o uso da Esperança do Desvio Preditivo (EPD; Expected Predictive Deviance) como uma medida bayesiana para a comparação e seleção entre diferentes modelos da TRI. Aliás, a comparação e seleção de modelos constitui um tema de grande potencial de pesquisa para a TRI, tanto no contexto da Inferência Bayesiana quanto freqüentista (clásssica).

Uma importante informação sobre o método DAGS é proveniente do trabalho de Sahu (2002) o qual revela que uma das grandes vantagens deste método é a eficiência quanto ao tempo de processamento computacional exigido pelo algoritmo, podendo ser até 10 vezes mais rápido quando comparado aos algoritmos MCMC que se baseiam em MetropolisHastings (Gilks et al., 1995; Patz; Junker, 1999a; Patz; Junker, 1999b). Este resultado, segundo ele, torna-se ainda melhor quando distribuições a priori mais informativas para os parâmetros de acerto casual podem ser obtidas e/ou quando tem-se disponível para análise dos dados um grande número de respondentes. Este último resultado, conforme experiências encontradas na literatura, certamente está associado à condições favoráveis para a estimação do parâmetro de acerto casual já que este é reconhecidamente um parâmetro de difícil estimação (no sentido de convergência) em qualquer processo de estimação (veja, por exemplo, Patz e Junker (1999b) e Andrade et al. (2000, Sec. 3.7)). Uma desvantagem do método DAGS é a produção de amostras mais autocorrelacionadas (Sahu, 2002; Chen et al., 2000), o que exige um cuidado especial no momento de se escolher uma "boa" sequência de valores gerados pela Cadeia de Markov, i.e., que resultem na seleção de valores aproximadamente "independentes" da distribuição a posteriori de interesse (ver comentários de Albert e Chib (1993, Sec. 2)).

\subsection{Metodologia Inferencial Adotada}

Diante da discussão apresentada na seção anterior e da leitura de pesquisas relacionadas não é difícil perceber a existência de várias limitações da Inferência Freqüêntista com relação ao desenvolvimento de modelos cada vez mais complexos; dentre os quais os mais recentes da TRI (e.g. Patz e Junker (1999b), Fox (2005), Bazán (2005), Azevedo (2008)). O desenvolvimento e o estudo de uma Teoria Geral de Espaços de Estados por Cadeias de Markov (Gelfand; Smith, 1990; Casella; George, 1992; Tierney, 1994; Chib; Greenberg, 1995) juntamente com o avanço tecnológico para a implementação de algoritmos computacionais têm sido decisivos para o avanço da Inferência Estatística Bayesiana. Aliado a isto, é inegável que a construção e a análise de modelos complexos podem ser realizados de maneira mais simples e natural quando considerados sob os métodos bayesianos via MCMC (Albert, 1992; Patz; Junker, 1999b) e, em especial, com o uso de dados aumentados (Albert, 1992; Béguin; Glas, 2001; Sahu, 2002; Bazán et al., 2006; Azevedo, 2008).

Sendo assim, consideraremos no desenvolvimento dos próximos capítulos a estimação e Inferência Bayesiana utilizando métodos MCMC para obter aproximação das distribui-

Matos, G. S.

$\mathrm{IME} / \mathrm{USP}$ 
ções a posteriori de alguns modelos da classe multidimensional da TRI a qual foi introduzida e discutida no presente capítulo sob um contexto bastante geral. Devemos dizer que atenção especial será dada aos métodos que utilizam técnicas de dados aumentados (Tanner; Wong, 1987; Albert, 1992; Albert; Chib, 1993; Béguin; Glas, 2001; Sahu, 2002; Fox, 2005; Bazán et al., 2006; Azevedo, 2008) tendo em vista a grande flexibilidade destes métodos para a modelagem de dados complexos bem como a relativa eficiência relacionada ao tempo computacional exigido para o processamento de algoritmos do tipo MCMC (Sahu, 2002).

Matos, G. S. 
Capítulo 3

\section{Modelos K-dimensionais da TRI e o Problema da Falta de Identificabilidade}

\subsection{Introdução}

Neste capítulo, apresentamos alguns dos modelos da TRI mais utilizados. Para o primeiro, aqui denominado K-dimensional de 2 Parâmetros Probito (Kd2PP), apresentamos uma discussão sobre algumas de suas similaridades e diferenças com relação a outros modelos da literatura Estatística, dentre eles, o Modelo de Regressão Multivariada Probito (RMVP; Chib (2000)) e os Modelos Lineares Generalizados Mistos (Rijmen et al., 2003). Ainda com relação a este modelo, procuramos discutir sobre o fato do mesmo aparecer na literatura sob várias formulações e denominações que eventualmente podem causar confusão entre pesquisadores e usuários em geral. Diante destas discussões sobre o modelo Kd2PP, também apresentamos um caso particular que por vezes é denominado Multidimensional Entre-Itens mas que por conveniência o denominaremos K-multivariado de 2 Parâmetros Probito (Km2PP). Apresentamos, ainda, o modelo Kd3PP (K-dimensional de 3 Parâmetros Probito) que surge como uma extensão direta do Kd2PP; concluindo assim a segunda seção do presente capítulo (Seção 3.2).

Após realizar um levantamento bibliográfico sobre a falta de identificabilidade dos modelos K-dimensionais da TRI, ficou claro a escassez de textos que viabilizem a compreensão de um tema que no desenvolvimento inferencial de acordo com Rivers (2003) “... é por vezes abordado de modo casual e inadequado". Diante desta situação, procuramos apresentar na Seção 3.3 uma discussão sobre a identificação e solução dos problemas causados pela falta de identificabilidade dos modelos K-dimensionais discutidos neste capítulo. Esta discussão é desenvolvida tanto no contexto da Inferência Clássica quanto Bayesiana e assim esperamos contribuir ainda mais para o desenvolvimento e o uso dos modelos K-dimensionais da TRI. Finalmente, na última seção do presente capítulo, Seção 3.4, apresentamos um resumo dos principais resultados encontrados e discutidos. 


\subsection{Modelos K-dimensionais com Função de Resposta ao Item Probito}

\subsubsection{Modelo K-dimensional de 2 Parâmetros Probito (Kd2PP)}

Na literatura estatística não é novidade que o modelo probito caracteriza-se pelo uso da função distribuição de uma variável normal padrão $\Phi($.$) para a modelagem de dados$ dicotômicos ou dicotomizados. No presente trabalho, o modelo K-dimensional de 2 parâmetros probito $(\mathrm{Kd} 2 \mathrm{PP})$ da TRI é obtido quando, na expressão (2.1), consideramos $F_{j}\left(\eta_{i j}\right)=\Phi\left(\eta_{i j}\right)$, com

$$
\eta_{i j}=-\beta_{1 j}+\boldsymbol{\theta}_{i}^{\prime} \boldsymbol{\beta}_{2 j}=\left(-1, \boldsymbol{\theta}_{i}^{\prime}\right)\left(\begin{array}{c}
\beta_{1 j} \\
\boldsymbol{\beta}_{2 j}
\end{array}\right)=\boldsymbol{\Theta}_{i}^{\prime} \boldsymbol{\beta}_{12 j},
$$

em que $\boldsymbol{\Theta}_{i} \in \mathbb{R}^{(1+K)}$ com $\beta_{1 j} \in \mathbb{R}$ e $\boldsymbol{\beta}_{2 j} \in \mathbb{R}^{K}$ sendo, respectivamente, os parâmetros associados às características de dificuldade e discriminação do item $j$.

Sob estas condições, segue que a verossimilhança (2.5) assume a seguinte forma associada ao modelo Kd2PP:

$$
L(\boldsymbol{\theta}, \boldsymbol{\beta} \mid \boldsymbol{y}, \boldsymbol{M}) \equiv P\left(\boldsymbol{Y} \mid \boldsymbol{\theta}, \boldsymbol{\beta}_{1}, \boldsymbol{\beta}_{2}\right)=\prod_{i=1}^{N} \prod_{j=1}^{J}\left\{\left[\Phi\left(\boldsymbol{\Theta}_{i}^{\prime} \boldsymbol{\beta}_{12 j}\right)\right]^{y_{i j}}\left[1-\Phi\left(\boldsymbol{\Theta}_{i}^{\prime} \boldsymbol{\beta}_{12 j}\right)\right]^{1-y_{i j}}\right\}^{m_{i j}}
$$

em que,

- $\boldsymbol{Y}$ - é a matriz $N \times J$ das respostas dicotômicas cuja $i$-ésima linha é $\boldsymbol{y}_{i}^{\prime}=\left(y_{i 1}, \ldots, y_{i j}\right.$, $\left.\ldots, y_{i J}\right)$;

- $\boldsymbol{\beta}_{1}=\left(\boldsymbol{\beta}_{11}, \ldots, \boldsymbol{\beta}_{1 j}, \ldots, \boldsymbol{\beta}_{1 J}\right)^{\prime}$ é um vetor $J$-dimensional de interceptos relacionados aos níveis de dificuldade dos $J$ itens (Reckase, 1997; Nojosa, 2002);

- $\boldsymbol{\beta}_{2}$ - é uma matriz $J \times K \operatorname{com} \boldsymbol{\beta}_{2 j}^{\prime}=\left(\beta_{2 j 1}, \ldots, \beta_{2 j k}, \ldots, \beta_{2 j K}\right)$ sendo a $j$-ésima linha correspondente ao vetor de $K$ parâmetros de discriminação associados ao poder discriminatório do $j$-ésimo item multidimensional (Reckase, 1997; Nojosa, 2002);

- $\boldsymbol{\theta}$ - é a matriz $N \times K$ cuja $i$-ésima linha corresponde ao traço latente (multidimensional) associado ao $i$-ésimo indivíduo, $\boldsymbol{\theta}_{i}^{\prime}=\left(\theta_{i 1}, \ldots, \theta_{i k}, \ldots, \theta_{i K}\right)$; e

- $\boldsymbol{\Theta}_{i}^{\prime} \boldsymbol{\beta}_{12 j}$ é tal como descrito em (3.1).

Com relação a este modelo, várias observações interessantes e importantes podem ser feitas. Em primeiro lugar, notamos que sob a condição dos vetores de traços latentes 
serem conhecidos (e.g., previamente estimados em testes anteriores), o mesmo pode ser visto como um caso especial do modelo de Regressão Multivariada Probito (RMVP) (Chib, 2000)) onde cada $\boldsymbol{\Theta}_{i}^{\prime}=\left(-1, \boldsymbol{\theta}_{i}^{\prime}\right)$ denota uma covariável comum aos $J$ itens; os parâmetros dos itens $\boldsymbol{\beta}_{12 j} \in R^{1+K}$ são os coeficientes de regressão e a probabilidade (condicional) de um vetor de respostas dicotômicas (marginalmente correlacionadas) pode ser fatorada em um produto de distribuições marginais probito devido à hipótese de independência condicional (Suposição (S2); Seção 2.1.1). O modelo Kd2PP também pode ser visto como pertencente a uma classe que extende a dos modelos lineares generalizados mistos pois, segundo Rijmen et al. (2003), o modelo Kd2PP só não pertence à classe linear generalizada mista porque a linearidade no preditor é "destruída" pelo produto entre os parâmetros de discriminação (efeitos fixos) e os traços latentes (considerados efeitos aleatórios). No caso em que os parâmetros de discriminação ou traços latentes são conhecidos fica claro, por exemplo, que toda teoria de estimação dos modelos lineares generalizados mistos pode ser utilizada. É importante observar ainda que o modelo Kd2PP tem surgido na literatura sob diversas denominações e formulações teóricas. McDonald (1997a), por exemplo, mostra que, a menos de uma parametrização, sua aproximação polinomial para a função de resposta ao item, $\Phi\left(\boldsymbol{\Theta}_{i}^{\prime} \boldsymbol{\beta}_{12 j}\right)$, é equivalente a de Christoffersson (1975) que foi desenvolvida sob o enfoque da análise fatorial para dados dicotomizados * . Já na área de análises políticas o modelo (3.2) é mais conhecido por modelo de votação espacial ("Spatial voting models") e sua obtenção (formulação) basea-se na construção de funções de utilidade com erros aleatórios (ver Clinton et al. (2004)). Nesta área de atuação podemos destacar Bailey (2001), Martin e Quinn (2002) e Quinn (2004) que propuseram alguns importantes avanços na aplicação do modelo de votação espacial (ou Kd2PP se preferir). Vale ressaltar que parte destes avanços deve-se a enorme flexibilidade obtida pela combinação entre a inferência bayesiana e os métodos de simulação Monte Carlo (Jackman, 2000).

Autores como te Marvelde et al. (2006) e Adams et al. (1997) referem-se ao modelo (3.2) por Multidimensional dentro-do-item ("within-item-multidimensional IRT model") tendo em vista que sua aplicação é adequada para a análise de testes em que cada item exige mais de um traço latente para ser respondido. Por outro lado, um caso particular deste último é denominado modelo Multidimensional entre Itens da TRI ("between-itemmultidimensional IRT model") o qual é mais adequado para a análise de um teste que pode ser dividido em testes menores (sub-testes ou escalas) onde, de fato, as respostas aos itens podem ser descritas por modelos unidimensionais. Matos (2001), utilizando outra formulação, também obteve este modelo porém o denominou modelo Multivariado da TRI. Neste contexto, é possível perceber que os autores freqüentemente mencionam o desenvolvimento de suas pesquisas com relação ao modelo multidimensional (dentro-doitem) quando de fato suas aplicações correspondem a um sub-modelo: o Multidimensional entre Itens ou Multivariado. Exemplos de trabalhos neste contexto são: te Marvelde et al.

* Comentários, interpretações e comparações sobre parametrizações alternativas do modelo Kd2PP também são apresentadas em McDonald (1997a).

Matos, G. S.

$\mathrm{IME} / \mathrm{USP}$ 
(2006, p. 7 e 15), Li e Schafer (2005, p. 6 e 10), de la Torre e Patz (2002, p. 4) e Segall (1996, p. 333 e 342 ).

Na próxima sub-seção apresentamos alguns detalhes do que preferimos denominar modelo Multivariado da TRI e, na sub-seção subseqüente, apresentamos uma extensão direta do modelo Kd2PP para o caso de 3 parâmetros.

\subsubsection{Modelo K-multivariado de 2 Parâmetros Probito (Km2PP)}

Para melhor compreender o modelo K-multivariado (multidimensional entre itens) é importante entender que, assim como o K-dimensional (multidimensional dentro-do-item), o modelo é adequado para a análise de um teste em que as respostas aos itens dependem de múltiplos traços latentes dos respondentes. Entretanto, este modelo é mais adequado para a análise de um teste em que dois ou mais constructos são analisados a partir de conjuntos de itens (provas) distintos(as); e.g.; um conjunto de itens (prova) de Português e outro(a) de Matemática. Neste contexto, o modelo K-multivariado pode ser desenvolvido tal como foi proposto por Matos (2001).

De outra forma e, matematicamente falando, o modelo K-multivariado, mantendo-se adequado à análise de vários testes (e.g. um de Língua Portuguesa e outro de Matemática), também pode ser visto como um sub-modelo do K-dimensional. Para perceber isto, considere $C_{1}, C_{2}, \ldots, C_{K}$ partição do conjunto dos índices dos itens de um teste multidimensional. Considere, ainda, a estrutura particular do modelo multidimensional em que somente um elemento do vetor de discriminação de cada item é não nulo, ou seja, em que o $j$-ésimo item só "discrimina" (ou "carrega") numa única dimensão $\theta_{i k}$ do vetor de traços latentes $\boldsymbol{\theta}_{i}^{\prime}=\left(\theta_{i 1}, \ldots, \theta_{i K}\right)$. Em outras palavras, podemos dizer que cada item só contribui para a informação sobre uma das dimensões do vetor de traços latentes de um indivíduo qualquer. Nestas condições e sem perda de generalidade, suponhamos que $\forall j \in C_{1}, \boldsymbol{\beta}_{2 j}^{\prime}$ é da forma $\left(\beta_{2 j 1}, 0,0, \ldots, 0\right), \forall j \in C_{2}, \boldsymbol{\beta}_{2 j}^{\prime}$ é da forma $\left(0, \beta_{2 j 2}, 0, \ldots, 0\right)$ e assim sucessivamente até que $\forall j \in C_{K}, \boldsymbol{\beta}_{2 j}^{\prime}$ é da forma $\left(0, \ldots, 0, \beta_{2 j K}\right)$. Segue, portanto, que a verossimilhança (3.2) pode ser reescrita como

$$
\begin{aligned}
L(\boldsymbol{\theta}, \boldsymbol{\beta} \mid \boldsymbol{y}) & \equiv P\left(\boldsymbol{Y} \mid \boldsymbol{\theta}, \boldsymbol{\beta}_{\mathbf{1}}, \boldsymbol{\beta}_{\mathbf{2}}\right)= \\
& =\prod_{i=1}^{N} \prod_{k=1}^{K} \prod_{j \in \boldsymbol{C}_{k}}\left\{\Phi\left(\theta_{i k} \beta_{2 j k}-\beta_{1 j k}\right)^{y_{i j k}}\left[1-\Phi\left(\theta_{i k} \beta_{2 j k}-\beta_{1 j k}\right]^{1-y_{i j k}}\right\}^{m_{i j k}},\right.
\end{aligned}
$$

em que:

- $\boldsymbol{Y}$ é a matriz $N \times J, J=\sum_{k=1}^{K} J_{k}$, de todas as respostas dicotômicas cuja $i$-ésima linha é $\boldsymbol{y}_{i}^{\prime}=\left(\boldsymbol{y}_{i .1}^{\prime}, \ldots, \boldsymbol{y}_{i . K}^{\prime}\right)=\left(y_{i 11}, \ldots, y_{i J_{1} 1}, \ldots, y_{i 1 K}, \ldots, y_{i J_{K} K}\right)$;

Matos, G. S.

IME/USP 
- $\boldsymbol{\beta}_{1}^{\prime}=\left(\boldsymbol{\beta}_{i .1}^{\prime}, \ldots, \boldsymbol{\beta}_{i . K}^{\prime}\right)=\left(\beta_{111}, \ldots, \beta_{1 J_{1} 1}, \ldots, \beta_{11 K}, \ldots, \beta_{1 J_{K} K}\right)$ é o vetor de dimensão $J$ correpondente aos interceptos relacionados aos níveis de dificuldade $\operatorname{dos} J$ itens;

- $\boldsymbol{\beta}_{2}$ - é uma matriz $J \times K \operatorname{com} \boldsymbol{\beta}_{2 j k}^{\prime}=\left(0, \ldots, \beta_{2 j k}, \ldots, 0\right)$ sendo a $j$-ésima linha correspondente à discriminação do item $j$ pertencente ao teste $k ; j=1, \ldots, J_{1}, \ldots, 1, \ldots, J_{K}$ e $k=1, \ldots, K$.

- $\boldsymbol{\theta}$ - é a matriz $N \times K$ de todos os traços latentes individuais de modo que a $i$-ésima linha é $\boldsymbol{\theta}_{i}^{\prime}=\left(\theta_{i 1}, \ldots, \theta_{i K}\right)$;

- $\boldsymbol{C}_{k}$ - é o conjunto dos índices dos itens pertencentes ao teste $k, k=1, \ldots, K$.

Como mencionado anteriormente a verossimilhança acima descrita coincide com a obtida por Matos (2001) e corresponde ao que preferimos denominar modelo K-multivariado de 2 Parâmetros Probito (Km2PP), onde $K$ denota a dimensão dos vetores dos traços latentes.

\subsubsection{Modelo K-dimensional de 3 Parâmetros Probito (Kd3PP)}

O modelo K-dimensional de 3 Parâmetros Probito (Kd3PP), algumas vezes utilizado na área educacional, incorpora, no modelo Kd2PP, a informação sobre a probabilidade dos respondentes com baixa habilidade responderem corretamente a um item de múltipla escolha (Andrade et al., 2000). Neste caso, a função de resposta ao item é dada por:

$$
P\left(Y_{i j}=y_{i j} \mid \boldsymbol{\theta}_{i}, \boldsymbol{\beta}_{j}\right)=\beta_{3 j}+\left(1-\beta_{3 j}\right) F_{j}\left(\eta_{i j}\right)=\beta_{3 j}+\left(1-\beta_{3 j}\right) \Phi\left(\eta_{i j}\right), \quad \forall i, j,
$$

em que $\beta_{3 j} \in[0,1]$ representa uma probabilidade de acerto casual ao item por parte dos respondentes com níveis baixo de habilidades.

Vale lembrar que o modelo K-dimensional de 1 parâmetro (da família Rasch) probito (Kd1PP) é obtido sob a restrição do parâmetro $\beta_{3 j}$ ser nulo, além dos valores de discriminação serem todos constantes e iguais.

\subsection{O Problema da Falta de Identificabilidade}

A falta de identificabilidade, discutida inicialmente na Seção 2.2.1, é um problema comumente encontrado nos modelos da classe Geral de Variáveis Latentes (Bartholomew, 1999). Nos modelos multidimensionais da TRI apresentados neste capítulo, por exemplo, é possível observar que existem pelo menos dois tipos de falta de identificabilidade, um devido à invariância à transformaçoes lineares e outro devido à rotações ortogonais (um

Matos, G. S.

IME/USP 
terceiro tipo é comentado no último capítulo desta tese, Cap 6). O primeiro tipo deve-se à invariância ̀̀ transformações lineares, pois se $\boldsymbol{\theta}_{i}^{*}=\boldsymbol{A} \boldsymbol{\theta}_{i}+\boldsymbol{b} \operatorname{com} \boldsymbol{A}$ e $\boldsymbol{b}$ sendo, respectivamente, uma matriz não-singular e um vetor de reais, então, para todo conjunto de dados amostrais, $\boldsymbol{y}$, vale que

$$
\begin{aligned}
\Phi\left(-\beta_{1 j}+\boldsymbol{\beta}_{2 j}^{\prime} \boldsymbol{\theta}_{i}\right) & =\Phi\left(-\beta_{1 j}+\boldsymbol{\beta}_{2 j}^{\prime} \boldsymbol{A}^{-1}\left(\boldsymbol{\theta}_{i}^{*}-\boldsymbol{b}\right)\right) \\
& =\Phi\left(-\left(\beta_{1 j}+\boldsymbol{\beta}_{2 j}^{\prime} \boldsymbol{A}^{-1} \boldsymbol{b}\right)+\boldsymbol{\beta}_{2 j}^{\prime} \boldsymbol{A}^{-1} \boldsymbol{\theta}_{i}^{*}\right) \\
& =\Phi\left(-\beta_{1 j}^{*}+\left(\boldsymbol{\beta}_{2 j}^{*}\right)^{\prime} \boldsymbol{\theta}_{i}^{*}\right),
\end{aligned}
$$

em que $\beta_{1 j}^{*}=\beta_{1 j}+\boldsymbol{\beta}_{2 j}^{\prime} \boldsymbol{A}^{-1} \boldsymbol{b}$ e $\boldsymbol{\beta}_{2 j}^{*}=\left(\boldsymbol{A}^{-1}\right)^{\prime} \boldsymbol{\beta}_{2 j}, \forall i, j$, implicando, portanto, em $L(\boldsymbol{\theta}, \boldsymbol{\beta} \mid \boldsymbol{y})=$ $L\left(\boldsymbol{\theta}^{*}, \boldsymbol{\beta}^{*} \mid \boldsymbol{y}\right)$.

Este tipo de não-identificabilidade é mais conhecido na TRI como um problema de indeterminação da escala de medida (veja Seção 2.2.1 e Andrade (2001, p. 15)) e por conta disso, é de fundamental importância que se estabeleça (fixe) uma métrica (escala de medida com localizaçao e escala como referência de posssíveis valores para os parâmetros) para um dos dois conjuntos de parâmetros, - itens ou traços latentes -, pois sem isto não há sentido algum em se falar sobre a estimação conjunta desses parâmetros.

Com o intuito de simplificar a compreensão de nossa discussão sobre as possíveis soluções dos problemas causados pela falta de identificabilidade, decidimos em um primeiro momento realizar a discussão sob o ponto de vista da inferência clássica ou freqüentista e, em seguida, sob o ponto de vista da inferência bayesiana, conforme veremos nas próximas sub-seções. Mas antes apresentamos as seguintes definições que poderão ser úteis à compreensão do texto e que podem ser vistas, por exemplo, em Rivers (2003).

Definição 1 (Parâmetros Observacionalmente Equivalentes). Dois pontos do espaço paramétrico $\boldsymbol{\Psi}_{1}, \boldsymbol{\Psi}_{2} \in \boldsymbol{\Theta}$ são ditos observacionalmente equivalentes se $p_{\boldsymbol{\Psi}_{1}}=p_{\boldsymbol{\Psi}_{2}}$.

A igualdade $p_{\boldsymbol{\Psi}_{1}}=p_{\boldsymbol{\Psi}_{2}}$ significa dizer que dois modelos probabilísticos são ponto a ponto iguais, ou seja, $p_{\boldsymbol{\Psi}_{1}}(\boldsymbol{y})=p_{\boldsymbol{\Psi}_{2}}(\boldsymbol{y})$ para todo valor amostral $\boldsymbol{y}$.

Definição 2 (Parâmetro e Modelo Globalmente Identificável). Um ponto do espaço paramétrico $\boldsymbol{\Psi}_{0} \in \boldsymbol{\Theta}$ é dito identificável ou globalmente identificável se não existe nenhum outro ponto do espaço paramétrico observacionalmente equivalente à $\Psi_{0}$. Neste caso, dizemos que o modelo probabilístico $p_{\Psi}$ é globalmente identificável.

Definição 3 (Parâmetro e Modelo Localmente Identificável). Um ponto do espaço paramétrico $\boldsymbol{\Psi}_{0} \in \boldsymbol{\Theta}$ é dito ser localmente identificável se existe uma vizinhança $\boldsymbol{V}$ de $\boldsymbol{\Psi}_{0}$ tal que não exista nenhum outro ponto do espaço paramétrico $\boldsymbol{\Psi} \in \boldsymbol{\Theta} \cap \boldsymbol{V}$ observacionalmente equivalente à $\boldsymbol{\Psi}_{0}$. Neste caso, dizemos que o modelo probabilístico $p_{\boldsymbol{\Psi}}$ é localmente identificável na vizinhança de $\mathbf{\Psi}_{0}$.

Matos, G. S.

$\mathrm{IME} / \mathrm{USP}$ 


\subsubsection{Não-Identificabilidade Sob o Ponto de Vista da Inferência Clássica}

\subsubsection{Traços Latentes como Efeitos Fixos}

Em geral, as propostas para o estabelecimento de uma métrica, ou seja, para a eliminação do problema de indeterminação da escala de medida nos modelos K-dimensionais da TRI, consistem em adotar uma normalização sobre um ou outro conjunto de parâmetros (dos itens ou traços latentes). Uma proposta (solução) bastante utilizada na prática que denominaremos normalização padrão, introduz no processo de estimação (freqüentista) uma origem e uma escala através das restrições:

$$
\sum_{i=1}^{N} \theta_{i k}=0, \quad \sum_{i=1}^{N} \theta_{i k}^{2}=N, \quad k=1, \ldots, K \text { e } \sum_{i=1}^{N} \theta_{i k} \theta_{i l}=0 \quad k \neq l .
$$

Entretanto, conforme Rivers (2003) notou, após adotar esta normalização, ainda nos resta a possibilidade de rotacionar os traços latentes (ou os parâmetros de discriminação) em torno da origem sem que os valores do modelo multidimensional se alterem, ou seja, Rivers (2003) notou que a normalização padrão é insuficiente para eliminar a falta de identificabilidade (veja também Jackman (2001)).

Este segundo tipo de não-identificabilidade é equivalente ao encontrado no tradicional modelo fatorial e é mais conhecido por invariância rotacional. Formalmente, se $\boldsymbol{R}$ é uma matriz ortogonal qualquer tal que $\boldsymbol{R}^{\prime} \boldsymbol{R}=\boldsymbol{R} \boldsymbol{R}^{\prime}=\boldsymbol{I}_{K}$ e $\boldsymbol{\theta}_{i}^{*}=\boldsymbol{R} \boldsymbol{\theta}_{i}$, então

$$
\Phi\left(-\beta_{1 j}+\boldsymbol{\beta}_{2 j}^{\prime} \boldsymbol{\theta}_{i}\right)=\Phi\left(-\beta_{1 j}+\boldsymbol{\beta}_{2 j}^{\prime} \boldsymbol{R}^{\prime} \boldsymbol{\theta}_{i}^{*}\right)=\Phi\left(-\beta_{1 j}+\left(\boldsymbol{\beta}_{2 j}^{*}\right)^{\prime} \boldsymbol{\theta}_{i}^{*}\right)
$$

$\operatorname{com} \boldsymbol{\beta}_{2 j}^{*}=\boldsymbol{R} \boldsymbol{\beta}_{2 j}, \quad \forall i, j$.

Diante deste novo tipo de não-identificabilidade e sem a possibilidade de obter novas informações dos dados para eliminá-la, uma das estratégias requer que se estabeleça restrições adicionais sobre os parâmetros do modelo. Vale ressaltar, porém, que esta tarefa não é tão simples quanto é para identificá-la (veja alguns exemplos em Skrondal e Rabe-Hesketh (2004)). No contexto dos modelos fatoriais, por exemplo, Algina (1980) e referências citadas no artigo apresentam contra-exemplos mostrando que determinadas soluções propostas na literatura não necessariamente identificam a matriz de cargas fatoriais, matriz esta que é equivalente a matriz dos parâmetros de discriminação $\boldsymbol{\beta}_{2}$. Do mesmo modo, Rivers (2003) chama a atenção para o fato de que as soluções propostas para a eliminação da falta de identificabilidade dos modelos da TRI também são delicadas e até mesmo controversas.

Um importante estudo sobre a falta de identificabilidade nos modelos multidimensionas da TRI, deve-se a Rivers (2003) que utilizando resultados da teoria da informação de Rothenberg (1971) e Bowden (1973), estabeleceu condições necessárias e suficientes 
para a identificabilidade local (ver Definição 3) de um modelo K-dimensional de 2 Parâmetros (Kd2P) com FRI qualquer, contínua e duplamente diferenciável. Neste contexto Rivers (2003) também mostrou que ao adotar a normalização padrão (3.6), com exceção do caso unidimensional, pelo menos $K(K-1) / 2$ restrições adicionais sobre os parâmetros dos itens são necessárias para remover a falta de identificabilidade rotacional. Acrescentandose, ainda, a condição de que as colunas da matrix de traços latentes, $\boldsymbol{\theta}$, sejam linearmente independentes e que não contenham o vetor unitário em seus espaços, além da hipótese de que a matrix de discriminção, $\boldsymbol{\beta}_{2}$, seja de posto completo ${ }^{\dagger}$. Com base nesta última estratégia para tornar um modelo multidimensional identificável com relação à localização, escala e rotação, propostas para restringir os parâmetros dos itens derivam de soluções análogas às da análise fatorial, pois, nestas análises, restrições sobre a matriz de cargas fatoriais podem ${ }^{\ddagger}$ ser adotadas com o intuito de obter uma única solução (estimativa) particular ao final do processo de estimação (Press, 2005; Lopes; West, 2004; Quinn, 2004; Jöreskog, 1969). É importante destacar ainda que Rivers (2003) também apresentou normalizações alternativas que tornam o modelo multidimensional localmente identificável sem a necessidade de restrições adicionais sobre parâmetros dos itens. Uma delas consiste em, além de admitir as hipóteses acima descritas sobre as matrizes $\boldsymbol{\theta}$ e $\boldsymbol{\beta}_{2}$, fixar $K+1$ pontos (traços latentes) independentes ou, de forma equivalente, considerar $K(K+1)$ restrições independentes sobre a matrix de traços latentes.

No que conscerne aos modelos unidimensionais da TRI, conforme já mencionado, Rivers (2003) mostrou que a normalização padrão é suficiente para se obter a identificabilidade local mas não a global (Definição 2) uma vez que não elimina a não-identificabilidade rotacional (direcional no caso do modelo unidimensional) a qual permite que as probabilidades de sucesso sejam inalteradas após a multiplicação dos valores dos traços latentes e parâmetros de discriminação por -1. Neste caso, uma identificabilidade global pode ser facilmente obtida se além da normalização padrão, a restrição de não-negatividade para os valores dos parâmetros de discriminação é adotada. Note que tal restrição é intuitiva e naturalmente aceitável na área educacional pois maiores probabilidades de resposta correta aos itens são esperadas dentre aqueles respondentes cujos traços latentes (habilidades) correspondem à valores positivos e de maiores magnitudes.

\subsubsection{Traços Latentes como Efeitos Aleatórios}

Até o momento, a discussão sobre a falta de identificabilidade foi desenvolvida considerando os vetores de traços latentes como efeitos fixos. Agora, com o intuito de realizar

$\dagger$ Note que esta última hipótese está diretamente associada à correta especificação da dimensionalidade do item.

$\ddagger$ Em alguns casos, restrições paramétricas adicionais não são previamente estabelecidas, de modo que o próprio método de estimação encontra uma solução (estimativa) particular que, em geral, é rotacionada com o intuito de simplificar as interpretações (Jöreskog, 1969; Bock et al., 1988).

Matos, G. S.

IME/USP 
a estimação dos parâmetros dos itens (e, possivelmente, de outros) sem a presença dos traços latentes é comum considerá-los como variáveis aleatórias ou efeitos aleatórios (ver discussão sobre parâmetros de incômodo na pág. 12) e, neste contexto, o processo de estimação e inferência sob o enfoque freqüentista basea-se exclusivamente na verossimilhança marginal dada por

$$
\begin{aligned}
L(\boldsymbol{\eta}, \boldsymbol{\beta} \mid \boldsymbol{y}) & \equiv P\left(\boldsymbol{Y} \mid \boldsymbol{\eta}, \boldsymbol{\beta}_{1}, \boldsymbol{\beta}_{2}\right) \\
& =\prod_{i=1}^{N} \int_{R^{K}} \prod_{j=1}^{J}\left\{\left[\Phi\left(\mathbf{\Theta}_{i}^{\prime} \boldsymbol{\beta}_{12 j}\right)\right]^{y_{i j}}\left[1-\Phi\left(\boldsymbol{\Theta}_{i}^{\prime} \boldsymbol{\beta}_{12 j}\right)\right]^{1-y_{i j}}\right\}^{m_{i j}} g(\boldsymbol{\theta} \mid \boldsymbol{\eta}) d \boldsymbol{\theta} \\
& =\prod_{i=1}^{N} \int_{R^{K}} L\left(\boldsymbol{\theta}, \boldsymbol{\beta} \mid \boldsymbol{y}_{i .}\right) g(\boldsymbol{\theta} \mid \boldsymbol{\eta}) d \boldsymbol{\theta},
\end{aligned}
$$

em que $g(\boldsymbol{\theta} \mid \boldsymbol{\eta})$ denota uma função densidade (arbitrária) dos vetores de traços latentes com parâmetros $\boldsymbol{\eta}$ e $L\left(\boldsymbol{\theta}, \boldsymbol{\beta} \mid \boldsymbol{y}_{i .}\right)=\prod_{j=1}^{J}\left\{\left[\Phi\left(\boldsymbol{\Theta}_{i}^{\prime} \boldsymbol{\beta}_{12 j}\right)\right]^{y_{i j}}\left[1-\Phi\left(\boldsymbol{\Theta}_{i}^{\prime} \boldsymbol{\beta}_{12 j}\right)\right]^{1-y_{i j}}\right\}^{m_{i j}}$ a função de verossimilhança condicional ${ }^{\S}$ associada ao $i$-ésimo respondente.

Colocado desta forma, fica simples perceber que o presente modelo pertence à classe Linear Geral de Variáveis Latentes discutida por Bartholomew (1999). Esta marginalização também pode ser considerada como uma formulação alternativa para se obter o mesmo modelo da análise fatorial para dados binários. Para maiores detalhes sobre este último fato sugerimos a leitura de Takane e de Leeuw (1987), McDonald (1982), Bock et al. (1988), Mellenbergh (1994), Reckase (1997b) e Bartholomew (1999).

Quando analisamos o modelo marginal, $P\left(\boldsymbol{Y} \mid \boldsymbol{\eta}, \boldsymbol{\beta}_{1}, \boldsymbol{\beta}_{2}\right)$, no contexto mais geral da classe Linear Geral de Variáveis Latentes, observamos que o mesmo está sujeito a uma arbitrariedade maior do que o modelo condicional $P\left(\boldsymbol{Y} \mid \boldsymbol{\theta}, \boldsymbol{\beta}_{1}, \boldsymbol{\beta}_{2}\right)$. Bartholomew (1999), por exemplo, mostra que ainda que transformações um-a-um sobre o espaço latente provoquem mudanças na distribuição dos traços latentes $g(\boldsymbol{\theta} \mid \boldsymbol{\eta})$ e/ou no modelo condicional $P\left(\boldsymbol{Y} \mid \boldsymbol{\theta}, \boldsymbol{\beta}_{1}, \boldsymbol{\beta}_{2}\right)^{\boldsymbol{\Phi}}$; o modelo marginal fica inalterado, pois isto corresponde a simples transformações de variáveis de modo que a integração em (3.8) não sofre alteração alguma. Para eliminar essa arbitrariedade, um procedimento análogo ao discutido para o modelo condicional pode ser adotado; ou seja; fixar de forma total ou parcial os parâmetros associados à localização e escala da distibuição (arbitrária) dos traços latentes $g(\boldsymbol{\theta} \mid \boldsymbol{\eta})$. Neste sentido, é comum supor que

$$
\boldsymbol{\theta}_{1}, \ldots, \boldsymbol{\theta}_{N} \stackrel{i . i . d}{\sim} N_{K}\left(\boldsymbol{\mu}_{\theta}, \boldsymbol{\Sigma}_{\theta}\right), \operatorname{com} \boldsymbol{\mu}_{\theta}=\mathbf{0} \text { e } \boldsymbol{\Sigma}_{\theta}=\boldsymbol{I}
$$

$\S$ Condicional com relação ao vetor aleatório $\boldsymbol{\theta}$ e não com relação a uma estatística suficiente que gera uma verossimilhança condicional no sentido de pseudo-verossimilhança (Pace; Salvan, 1997; Andersen, 1980).

I No caso particular apresentado neste capítulo, transformações lineares ou rotacionais não modificam o modelo condicional $P\left(\boldsymbol{Y} \mid \boldsymbol{\theta}, \boldsymbol{\beta}_{1}, \boldsymbol{\beta}_{2}\right)$.

Matos, G. S.

IME/USP 
Entretanto, assim como no caso do modelo condicional discutido na sub-seção anterior; esta condição continua sendo insuficiente para eliminar a falta de identificabilidade, pois tanto o modelo condicional $P\left(\boldsymbol{Y} \mid \boldsymbol{\theta}, \boldsymbol{\beta}_{1}, \boldsymbol{\beta}_{2}\right)$ como a distribuição $g(\boldsymbol{\theta} \mid \boldsymbol{\eta})$ são invariantes à transformações ortogonais (rotações) (Jackman, 2001; Rivers, 2003) e, portanto, a verossimilhança marginal continua não-identificável. Novamente, as soluções são obtidas através de restrições adicionais sobre os parâmetros do modelo. Uma proposta apresentada por Béguin e Glas (2001) e de autoria de Fraser (1988), requer que os parâmetros dos itens sejam fixados da seguinte forma: $\beta_{2 j k}=0$, para $j=1, \ldots, K-1$ e $k=j+1, \ldots, K$. De modo equivalente, Béguin e Glas (2001) propuseram fixar $K$ parâmetros de dificuldade, $\beta_{1 j}=0$ e $\beta_{2 j k}=I(j=k)$ para $j, k=1, \ldots, K$, onde $I($.$) é a função indicadora usual.$ Esta última proposta, apresentada num contexto bayesiano, possui a vantagem de não exigir restrições sobre os parâmetros populacionais $\boldsymbol{\mu}$ e $\boldsymbol{\Sigma}_{\theta}$ e uma alternativa à condição de $K$ parâmetros de dificuldade nulos é adotar $\boldsymbol{\mu}_{\theta}=\mathbf{0}$ (Holman; Glas, 2005). Soluções tais como as discutidas na sub-seção anterior também podem ser adotadas.

Diante da invariância rotacional mencionada acima, tanto por parte do modelo de resposta ao item (modelo amostral/verossimilhança) quanto da distribuição normal multivariada atribuída aos traços latentes, devemos dizer que a proposta do presente trabalho de se considerar uma distribuição normal (ou t) multivariada assimétrica para os traços latentes surge como uma solução natural para a eliminação de tal invariância, pois as mesmas são variantes à transformações rotacionais (Sahu et al., 2003). Devemos dizer, ainda, que uma das principais vantagens desta solução segue do fato de não exigir a adoção de restrições adicionais sobre os parâmetros dos itens. Detalhes sobre esta proposta são apresentadas no desenvolvimento dos Capítulos 4 e 5.

Para concluir a discussão sobre a falta de identificabilidade no contexto da inferência clássica via verossimilhança marginal é importante dizer que alguns autores não adotam restrições adicionais para resolver o problema da invariância rotacional. Bock e Aitkin (1981), por exemplo, após desenvolverem o método de estimação por máxima verossimilhança marginal via algoritmo EM, lembram que a solução (estimativas) obtida sem o uso de restrição adicional sobre os parâmetros dos itens não é única e depende dos valores adotados para a inicialização do algoritmo. Eles lembram, ainda, que apesar dos métodos rotacionais, aplicados após a obtenção da estimativa da matriz de discriminação, permitirem a obtenção de soluções (estimativas) distintas, equivalentes e de mais fácil interpretação; as estatísticas de teste para o ajuste do modelo permanecem únicas. Isto, de certo modo, já era esperado, tendo em vista que tais estatísticas dependem da verossimilhança marginal que é invariante à rotação. Jöreskog (1969, p. 195), no contexto da análise fatorial tradicional, também admite que as estimativas obtidas para as cargas fatoriais correspondem a uma solução arbitrária para a qual o algoritmo computacional conseguiu convergir e por isso a matriz de cargas fatoriais estimada pode ser convenientemente rotacionada de forma ortogonal ou oblíqua. Outros autores; apesar de procederem de maneira análoga; não deixam claro quais condições influenciam e garantem a conver-

Matos, G. S.

$\mathrm{IME} / \mathrm{USP}$ 
gência do algoritmo computacional para uma estimativa particular dos parâmetros (veja, por exemplo, Bock et al. (1988)). Para estes casos é importante dizer que os cálculos dos erros-padrões requerem maiores cuidados pois sem restrições suficientes o máximo não é único e a matriz de informação será singular (Bartholomew, 1999). Métodos de simulação, tais como "bootstrap" são possíveis alternativas para a obtenção de erros-padrões em tais situações (veja, por exemplo, Shao e Tu (1995)).

\subsubsection{Não-Identificabilidade Sob o Ponto de Vista da Inferência Bayesiana}

A ausência de dificuldades em uma análise bayesiana na presença de parâmetros não identificáveis sempre é possível quando distribuições a priori próprias, ou seja, funções nãonegativas que integram 1, são adotadas para todos os parâmetros. Esta idéia é comumente citada na literatura bayesiana devido ao famoso comentário de Lindley (1971, p.46):

"In passing it might be noted that unidentifiability causes no real difficulty in the Bayesian approach".

A ausência de dificuldades a que nos referimos segue do fato de que a existência de uma distribuição a posteriori própria fica garantida quando distribuições a priori próprias são adotadas. Apesar disto, em estudos como o de Poirier (1998) e O'Hagan (1994) encontramos a indicação de que nem tudo está resolvido pois na presença de modelos não-identificáveis existem quantidades paramétricas para as quais os dados são nãoinformativos, ou seja, para as quais as distribuições marginais a priori e a posteriori são idênticas. Para melhor discutir esta questão bem como outras relacionadas a existência (se própria ou imprópria) da distribuição a posteriori na presença de uma verossimilhança não-identificável, apresentamos a seguinte definição de parâmetro e verossimilhança nãoidentificável:

Definição 4 (Parâmetro e Verossimilhança não-identificável). Considere $\Psi=$ $\left(\phi_{1}, \phi_{2}\right) \in \Theta$ um vetor de parâmetros em que $\phi_{1}$ e $\phi_{2}$ possivelmente também são vetores. Dizemos que $\phi_{1}$ é um parâmetro identificável e que $\phi_{2}$ é não-indentificável se para todo conjunto de dados amostrais, $\boldsymbol{y}$,

$$
L\left(\Psi^{(1)} ; \boldsymbol{y}\right)=L\left(\Psi^{(2)} ; \boldsymbol{y}\right)
$$

implica $\phi_{1}^{(1)}=\phi_{1}^{(2)}$ e $\phi_{2}^{(1)} \neq \phi_{2}^{(2)}$. Neste caso, dizemos ainda que a verossimilhança $L(\boldsymbol{\Psi} ; \boldsymbol{y})$ é não-identificável.

A partir desta definição observamos que o valor de uma verossimilhança não-identificável independe do valor assumido pelo parâmetro não-identificável $\phi_{2}$ de modo que podemos reescrever a verossimilhança em função apenas do parâmetro identificável $\phi_{1}$, ou seja,

Matos, G. S.

$\mathrm{IME} / \mathrm{USP}$ 
$L\left(\phi_{1} ; \boldsymbol{y}\right) \equiv L(\Psi ; \boldsymbol{y})$, ainda que o espaço de $\phi_{1}$ dependa de $\phi_{2}$ (Poirier, 1998; O'Hagan, 1994). Note que ao reescrever a verossimilhança desta forma podemos ser induzidos a pensar que a distribuição a priori é a única fonte de informação disponível sobre o parâmetro não-identificável $\phi_{2}$. Entretanto, Poirier (1998), após introduzir os conceitos de dados marginalmente e condicionalmente não-informativos para o parâmetro não-identificável $\phi_{2}$ ", mostrou que os dados podem sim (mas não necessariamente) fornecer informação sobre a distribuição marginal do parâmetro não-identificável $\phi_{2}$, desde que exista dependência a priori entre $\phi_{1}$ e $\phi_{2}$ (ver exemplos em Poirier (1998)). Por outro lado, independência a priori entre $\phi_{1}$ e $\phi_{2}$ é uma condição suficiente para que os dados sejam marginalmente não-informativos para $\phi_{2}$ (Proposição 1; Poirier (1998)). Este resultado é surpreendente, pois ainda que a especificação de distribuições a priori próprias para todos os parâmetros de um modelo não-identificável permita realizar inferência bayesiana, é possível que tal inferência seja sem efeito no sentido de que a informação (marginal) a posteriori sobre um parâmetro não-identificável seja a mesma a priori, ou seja, no sentido de que os dados podem continuar sem fornecer informação alguma sobre um parâmetro não-identificável.

Para continuar nossa discussão sobre não-identificabilidade no contexto da inferência bayesiana apresentamos neste momento o conceito de não-identificabilidade bayesiana formalizado por Dawid (1979), a saber:

Definição 5 (Não-identificabilidade Bayesiana). Considere um modelo Bayesiano constituído pela verossimilhança $L\left(\phi_{1}, \phi_{2} ; \boldsymbol{y}\right)$ e uma distribuição a priori $f\left(\phi_{1}, \phi_{2}\right)=$ $f\left(\phi_{1}\right) f\left(\phi_{2} \mid \phi_{1}\right)$. Então o parâmetro $\phi_{2}$ é dito não-indentificável se

$$
f\left(\phi_{2} \mid \phi_{1}, \boldsymbol{y}\right)=f\left(\phi_{2} \mid \phi_{1}\right)
$$

Neste caso, o modelo Bayesiano $f\left(\phi_{1}, \phi_{2} \mid \boldsymbol{y}\right) \propto L\left(\phi_{1}, \phi_{2} ; \boldsymbol{y}\right) p\left(\phi_{1}\right) p\left(\phi_{2} \mid \phi_{1}\right)$ também é dito ser não-identificável.

Com relação a este conceito é possível observar que o conceito apresentado por Poirier (1998) de dados condicionalmente não-informativos para o parâmetro não-identificável $\phi_{2}$ é exatamente o de não-identificabilidade bayesiana (ver também O'Hagan (1994)). Neste contexto, autores como Poirier (1998, Proposição 2), Dréze (1974) e Dréze e Richard (1983) observaram que na presença de uma verossimilhança não-identificável os dados sempre serão condicionalmente não-informativos para o parâmetro não-identificável $\phi_{2}$. $\mathrm{O}$ que de fato ocorre é que os conceitos de não-identificabilidade clássica e bayesiana são equivalentes, pois note que

$$
f\left(\phi_{2} \mid \phi_{1}, \boldsymbol{y}\right) \propto L\left(\phi_{1}, \phi_{2} ; \boldsymbol{y}\right) f\left(\phi_{2} \mid \phi_{1}\right) f\left(\phi_{1}\right)
$$

|| Os dados $\boldsymbol{y}$ são marginalmente não-informativos para o parâmetro não-identificável $\phi_{2}$ se e somente se $f\left(\phi_{2} \mid \boldsymbol{y}\right)=f\left(\phi_{2}\right)$. De modo análogo, os dados $\boldsymbol{y}$ são condicionalmente não-informativos para o parâmetro não-identificável $\phi_{2}$ se e somente se $f\left(\phi_{2} \mid \phi_{1}, \boldsymbol{y}\right)=f\left(\phi_{2} \mid \phi_{1}\right)$.

Matos, G. S.

$\mathrm{IME} / \mathrm{USP}$ 
de modo que esta proporcionalidade permite chegar à igualdade (3.10) se e somente se a verossimilhança for não-identificável, ou seja, $L\left(\phi_{1}, \phi_{2} ; \boldsymbol{y}\right)=L\left(\phi_{1} ; \boldsymbol{y}\right)$ (Gelfand; Sahu, 1999; Ghosh et al., 2000; O’Hagan, 1994).

Diante desta discussão, podemos perceber que o conceito de não-identificabilidade independe da natureza das distribuições a priori (Gelfand; Sahu, 1999) mas somente da natureza da verossimilhança; se identificável ou não. Apesar disto, percebemos que a escolha de distribuições a priori na presença de uma verossimilhança não-identificável ainda desempenha um papel importante na inferência bayesiana, pois ainda que a distribuição condicional a priori de um parâmetro não-identificável $\phi_{2}$ (dado $\left.\phi_{1}\right)$ nunca seja atualizada pelos dados, a escolha adequada de distribuições a priori possibilita, por exemplo, que a distribuição marginal a posteriori do parâmetro não-identificável $\phi_{2}$ seja atualizada pelos dados. Com relação a este último ponto, já vimos que uma proposta é adotar distribuições a priori dependentes para os parâmetros $\phi_{1}$ e $\phi_{2}$, sem esquecer que isto constitui apenas uma condição necessária (Proposição 1; Poirier (1998)). No contexto em que a inferência bayesiana é desenvolvida por métodos MCMC, Gelfand e Sahu (1999) recomendam que a distribuição a priori do parâmetro não-identificável $\phi_{2}$ seja moderadamente informativa pois a especificação de uma distribuição a priori insuficientemente informativa para o parâmetro $\phi_{2}$ possibilita a ocorrência de valores extremos na sequência gerada pela cadeia de Markov, o que dificulta a análise de convergência de tal cadeia. Por outro lado, o uso de uma distribuição a priori demasiadamente informativa não permite que os dados forneçam informação para o parâmetro $\phi_{2}$. Com o intuito de especificar a distribuição a priori moderadamente informativa para o parâmetro não-identificável $\phi_{2}$, Gelfand e Sahu (1999) lembram, ainda, que um número considerável de estudos por meio de dados simulados pode ser necessário. Finalmente, uma proposta alternativa e mais prática para a especificação de distribuições a priori quando um modelo é não-identificável, consiste em restringir de modo adequado alguns parâmetros do modelo à constantes, tal como no contexto da inferência clássica e especificar distribuições a priori para os demais parâmetros (veja, e.g.,Lopes e West (2004), Quinn (2004)).

De volta para o contexto dos modelos multidimensionais da TRI e, em particular, o Kd2PP, observamos a necessidade de se reescrever a verossimilhança (3.2) como função de vetores de parâmetros identificáveis $\phi_{1}$ e não-identificáveis $\phi_{2}$; tal como na Definição 4. Para isto precisamos obter uma relação 1 a 1 entre o vetor de parâmetros $(\boldsymbol{\theta}, \boldsymbol{\beta})$ e $\left(\phi_{1}, \phi_{2}\right)$. Diante de um problema análogo, Ghosh et al. (2000) conseguiram expressar esta relação 1 a 1 de maneira relativamente simples para o caso dos modelos de resposta ao item de 1 (um) parâmetro (Rasch, 1960/1980). Devemos dizer, entretanto, que esta relativa facilidade em se reescrever a verossimilhança em função dos parâmetros $\phi_{1}$ e $\phi_{2}$ não ocorre no caso do modelo Kd2PP pois a relação não-linear entre os traços latentes e os parâmetros associados à discriminação inviabilizam tal tarefa. Diante de tal situação, procuramos refletir sobre as possíveis vantagens de tal resultado e chegamos à conclusão de que de fato não teríamos grandes vantagens. Pois, conforme já mencionado, o resultado

Matos, G. S.

IME/USP 
da Proposição 1 de Poirier (1998) estabelece uma condição necessária mas não suficiente para que o parâmetro não-identificável $\phi_{2}$ possua uma distribuição marginal a posteriori atualizável pelos dados. Além disso, em modelos complexos como os que estamos tratando no presente trabalho, não há como saber analiticamente se tal distribuição marginal é ou não atualizável pelos dados, pois não temos disponível uma forma analítica fechada para ela.

\subsubsection{Prioris Impróprias e Verossimilhanças Não-Identificáveis}

$\mathrm{Na}$ inferência bayesiana freqüentemente encontramos o uso de distribuições a priori impróprias para expressar um estado de "ignorância" sobre uma ou mais quantidades de interesse não observáveis. Tal procedimento, constitui um perigo natural para uma análise bayesiana pois a distribuição a posteriori resultante também poderá ser imprópria. Poirier (1998), por exemplo, cita várias referências que exemplificam isto. Neste sentido, não é surpresa que a combinação de modelos não-identificáveis com distribuições a priori impróprias também possa levar à não-existência da distribuição a posteriori e conseqüetemente à inferências desastrosas. Atenção para que situações como estas não ocorram deve ser ainda maior quando uma forma analítica fechada para a distribuição a posteriori é de difícil obtenção e métodos MCMC são utilizados. Hobert e Casella (1996), por exemplo, mostraram que valores "amostrais" aparentemente bons podem ser obtidos mesmo quando a cadeia de Markov é nula; ou seja; quando a distribuição a posteriori de fato não existe (é imprópria). Neste contexto, Ghosh et al. (2000) apresentaram um importante Lema no qual condições necessárias e suficientes são estabelecidas para que uma distribuição a posteriori seja própria na presença de um modelo amostral não-identificável. Devido a sua importância, este Lema é reproduzido em seguida.

Lema 1. Considere o vetor de parametros $\left(\phi_{1}, \phi_{2}\right)$, onde $\phi_{1}$ e $\phi_{2}$ possivelmente também são vetores. Se $\boldsymbol{Y}$ tem uma função densidade de probabilidade não-identificável $f\left(\boldsymbol{y} \mid \phi_{1}, \phi_{2}\right)=f\left(\boldsymbol{y} \mid \phi_{1}\right)$, então para uma distribuição a priori $\pi\left(\phi_{1}, \phi_{2}\right)$, a distribuição a posteriori $\pi\left(\phi_{1}, \phi_{2} \mid \boldsymbol{y}\right)$ será própria se e somente se $\pi\left(\phi_{1} \mid \boldsymbol{y}\right)$ e $\pi\left(\phi_{2} \mid \phi_{1}\right)$ forem próprias.

Como podemos perceber, este Lema requer que a verossimilhança não-identificável seja escrita como função de parâmetros identificáveis e não-identificáveis (Definição 4) e, conforme discussão constante no último parágrafo da Seção anterior, o uso deste Lema em favor da inferência bayesiana dos modelos de resposta ao item K-dimensionais é algo extremamente difícil ou até mesmo impossível devido à não-linearidade entre os parâmetros (Rijmen et al., 2003).

Matos, G. S.

$\mathrm{IME} / \mathrm{USP}$ 


\subsection{Considerações sobre o Capítulo}

Neste capítulo, um dos modelos de resposta ao item mais importantes da literatura recente da TRI foi apresentado, o K-dimensional de 2 Parâmetros Probito (Kd2PP) (Reckase, 1997b; Segall, 2002; te Marvelde et al., 2006). Nesta ocasião também foram apresentados aspectos como a relação com outros modelos estatísticos, a existência de formulações alternativas para se chegar ao modelo e um estudo bibligráfico sobre a falta de identificabilidade deste modelo. Com relação a este último assunto é importante dizer que as idéias (e soluções) discutidas não se limitam ao modelo K-dimensional do tipo Probito mas também são igualmente válidas para o caso em que o modelo K-dimensional de resposta ao item basea-se na função de ligação do tipo logit ou outra qualquer (Rivers, 2003).

A partir das discussões desenvolvidas na Seção 3.3 é possível observar que a falta de identificabilidade é, em essência, um problema causado pela superparametrização do modelo amostral adotado, ou melhor, da função de verossimilhança. Também é possível observar que uma das melhores estratégias para se eliminar a falta de identificabilidade consiste em se restringir de forma adequada alguns parâmetros do modelo amostral (verossimilhança) e assim proceder a estimação dos demais parâmetros, seja por procedimentos da inferência clássica ou bayesiana (Fraser (1988), Béguin e Glas (2001), Quinn (2004), Lopes e West (2004)). Neste contexto, observamos que a invariância rotacional é o tipo de identificabilidade do modelo Kd2PP que apresenta maior dificuldade para ser eliminado e/ou controlado pois apesar de existirem várias soluções propostas na literatura muitas estão desprovidas de demontrações analíticas que comprovem a eficácia de tais soluções. Algina (1980), no contexto do modelo fatorial de variáveis contínuas, por exemplo, demonstrou que o estabelecimento de algumas condições para eliminar a invariância rotacional - frequiêntemente adotadas no processo de estimação dos modelos K-dimensionais da TRI- podem constituir condições necessárias mas não suficientes. Já no contexto do modelo K-dimensional da TRI o trabalho desenvolvido por Rivers (2003) tem muito a contribuir nesta área pois utilizando resultados da teoria da informação de Rothenberg (1971) e Bowden (1973), ele estabeleceu condições necessárias e suficientes para a identificabilidade local de um modelo K-dimensional de 2 Parâmetros (Kd2P) com FRI qualquer, contínua e duplamente diferenciável.

Uma das principais contribuições do presente capítulo é a disponibilidade de um texto que procura discutir de forma detalhada como é possível identificar e solucionar problemas que surgem no processo de estimação e inferência (clássica ou bayesiana) do modelo Kd2PP devido à indeterminação da escala de medida e invariância rotacional. Neste sentido, uma contribuição imediata é refletida no trabalho desenvolvido no Capítulo 5 onde somos capazes de melhor compreender e solucionar os problemas que ocorrem no processo de estimação bayesiana (via método de dados aumentados) quando uma distribuição normal multivariada assimétrica é adotada para representar o comportamento dos traços latentes, $\boldsymbol{\theta}_{i}, i=1, \ldots, N$. O uso desta distribuição a priori representa uma extensão direta do

Matos, G. S.

$\mathrm{IME} / \mathrm{USP}$ 
trabalho desenvolvido por Béguin e Glas (2001) resultando, assim, no que denominamos modelo K-dimensional de 2 Parâmetros Probito com Distribuição de Traços Latentes Normal Assimétrica (Kd2PP-NA).

No próximo capítulo apresentamos todo o desenvolvimento teórico necessário à implementação computacional do método de estimação de Dados Aumentado por Amostrador de Gibss (DAGS) do modelo K-dimensional de 3 Parâmetros Probito com Distribuição de Traços Latentes t-Assimétrica (Kd2PP-tA) o qual tem como caso particular o modelo Kd2PP-NA.

Matos, G. S. 
Capítulo 4

\section{Modelo Kd3PP (e Km3PP) com Distribuições Assimétricas para os Traços Latentes}

\subsection{Introdução}

No capítulo anterior vimos como é fundamental a adoção de uma métrica (localização e escala) para o desenvolvimento de um processo de estimação dos modelos multidimensionais da TRI, seja sob o paradigma freqüentista ou bayesiano. Neste contexto, Bartholomew (1999, Sec. 2.17) lembra que "... a escolha de uma distribuição normal padrão para os traços latentes é uma simples questão de convenção e adotada largamente por grande conveniência.". Bartholomew diz ainda que "...em efeito, os parâmetros dos itens são calibrados com relação à distribuição a priori padronizada daquela particular população e a escolha da forma normal é então equivalente à adotar uma particular escala.".

Como já era esperado, a distribuição normal padrão (uni ou multivariada) é a mais utilizada na modelagem e/ou no desenvolvimento dos métodos de estimação dos modelos de resposta ao item e dentre outras justificativas seu uso encontra apoio em resultados assintóticos que indicam pouca influência da distribuição (distribuição a priori, no contexto bayesiano) dos traços latentes sobre a distribuição marginal dos dados (distribuição marginal a posteriori, no contexto bayesiano) na medida em que a quantidade de itens aumenta. Alguns dos poucos trabalhos desenvolvidos nesta área para a TRI são devidos à Chang e Stout (1993), Chang (1996) e Froelich (2000) e um ponto importante a ser lembrado é que o desenvolvimento destes resultados levam em consideração o fato de que as variáveis respostas não são identicamente distribuídas. Não obstante a estes resultados, ainda podemos observar na literatura a necessidade de novas pesquisas nesta área, especialmente quando existe alguma evidência empírica de que a verdadeira distribuição dos traços latentes é assimétrica. Como exemplo, podemos citar a situação em que respondentes a um determinado teste são de alguma forma pré-selecionados e um dos objetivos é estimar os vetores de traços latentes individuais (Tavares (2001, Sec. 10.2 e 10.9), Matos (2001, Cap. 8) e Azevedo (2003, Sec. 10.2)). 
Motivados pela necessidade de melhor representar os fenômenos da área psicométrica* e da atual disponibilidade de distribuições elípticas assimétricas cujas propriedades são tão convenientes quanto aquelas devidas à distribuição normal (Branco; Arellano-Valle, 2004; Genton, 2004; Arellano-Valle et al., 2006), nosso objetivo na próxima seção é estender o trabalho de Béguin e Glas (2001) no sentido de desenvolver o método de estimação bayesiana dos modelos multidimensionais da TRI via DAGS (Dados Aumentados com Amostrador de Gibbs) para o caso em que os vetores de traços latentes comportam-se segundo uma distribuição multivariada t-Assimétrica. Sob outro ponto de vista nosso objetivo visa extender o trabalho de Bazán (2005) para o caso de traços latentes multidimensionais.

\subsection{Estimação Bayesiana do Modelo Kd3PP-tA (e Km3PP) via Método DAGS}

Nesta seção, nosso objetivo será descrever como o método de estimação bayesiana via DAGS (Dados Aumentados com Amostrador de Gibbs) pode ser aplicado ao modelo Kd3PP-tA. Neste contexto não é demais lembrar que todo procedimento a ser descrito é diretamente aplicável ao modelo Km3PP-tA uma vez que tal modelo é, ao menos em termos de modelagem probabilística, um sub-modelo do Kd3PP-tA (ver Seção 3.2.2 e item (c) da Sub-seção 4.2.4.2).

\subsubsection{Verossimilhança Aumentada}

A idéia e a formalização da abordagem de dados aumentados é freqüentemente creditada à Tanner e Wong (1987) pois com base em problemas de dados perdidos (veja também Dempster et al. (1977)) propuseram a introdução no conjunto de dados observados, $\boldsymbol{y}$, um novo conjunto de variáveis não observadas (ou perdidas) $\boldsymbol{z}$ e, a partir daí, a estimação da distribuição a posteriori utilizando os dados aumentados $D=(\boldsymbol{y}, \boldsymbol{z})$. Com este intuito, Tanner e Wong (1987) desenvolveram o algoritmo de dados aumentados a partir da seguinte identidade:

$$
p(\boldsymbol{\theta} \mid \boldsymbol{y})=\int p(\boldsymbol{\theta} \mid \boldsymbol{y}, \boldsymbol{z}) p(\boldsymbol{z} \mid \boldsymbol{y}) d \boldsymbol{z}
$$

Apesar do método de dados aumentados de Tanner e Wong (1987) ter como caso particular o amostrador de Gibbs (Liu, 2001, p. 137), acreditamos que a combinação entre o método de dados aumentados e o de Gibbs pode ser melhor compreendida quando reescrevemos a verossimilhança $L(\boldsymbol{\theta} \mid \boldsymbol{y})$ em função de uma verossimilhança de dados aumentados

* Heinen (1996, p. 105), por exemplo, ressaltou que a especificação da distribuição normal para as variáveis latentes em uma análise de dados de atitude, em geral, não é uma escolha muita adequada para descrever a distribuição populacional.

Matos, G. S.

$\mathrm{IME} / \mathrm{USP}$ 


\subsection{Estimação Bayesiana do Modelo Kd3PP-tA (e Km3PP) via Método} DAGS

$L(\boldsymbol{\theta} \mid \boldsymbol{y}, \boldsymbol{z})$ e não a distribuição a posteriori $p(\boldsymbol{\theta} \mid \boldsymbol{y})$ em função da distribuição a posteriori de dados aumentados $p(\boldsymbol{\theta} \mid \boldsymbol{y}, \boldsymbol{z})$. Neste sentido, achamos conveniente dizer que a idéia e a formalização da abordagem de dados aumentados deve-se à Dempster et al. (1977).

Na TRI, Albert (1992) foi pioneiro ao propor um método de estimação bayesiana com o uso de dados aumentados combinado com o amostrador de Gibbs (Geman; Geman, 1984; Gelfand; Smith, 1990) e, desde então, esta metodologia tem sido satisfatoriamente aplicada à vários modelos da TRI (ver, por exemplo, Sahu (2002) e Béguin e Glas (2001)). A idéia para combinar o método de dados aumentados com o amostrador de Gibbs, denominado daqui por diante DAGS, consiste em substituir a verossimilhança de dados observados, $L(\boldsymbol{\theta} \mid \boldsymbol{y})$, por uma denominada verossimilhança de dados aumentados, $L(\boldsymbol{\theta} \mid \boldsymbol{y}, \boldsymbol{z})$, que deve estar intrinsicamente ligada à de dados observados. Neste contexto, a seguinte igualdade é de fundamental importância para compreender como podemos obter uma verossimilhança aumentada associada ao modelo Kd3PP que depende dos parâmetros $\boldsymbol{\theta}$ e $\boldsymbol{\beta}$ :

$$
L(\boldsymbol{\theta}, \boldsymbol{\beta} \mid \boldsymbol{y})=\int L(\boldsymbol{\theta}, \boldsymbol{\beta} \mid \boldsymbol{y}, \boldsymbol{z}) d \boldsymbol{z}=\int p(\boldsymbol{y} \mid \boldsymbol{z}, \boldsymbol{\theta}, \boldsymbol{\beta}) p(\boldsymbol{z} \mid \boldsymbol{\theta}, \boldsymbol{\beta}) d \boldsymbol{z}
$$

O interessante desta igualdade é que ela nos remete à idéia de um método bastante utilizado na geração de números aleatórios o qual é conhecido por método da composição (veja, por exemplo, Tanner (1996)). Este método tem como idéia básica podermos amostrar um valor $\boldsymbol{y}$ a partir de um experimento sequencial, ou seja, amostrar primeiramente um valor $\boldsymbol{z}$ de uma distribuição preditiva condicional $p(\boldsymbol{z} \mid \boldsymbol{\theta}, \boldsymbol{\beta})$ e em seguida $\boldsymbol{y}$ da distribuição $p(\boldsymbol{y} \mid \boldsymbol{z}, \boldsymbol{\theta}, \boldsymbol{\beta})$. Observe, portanto, que a idéia de realizar inferência sobre os parâmetros $\boldsymbol{\theta}$ e $\boldsymbol{\beta}$ a partir de uma verossimilhança de dados aumentados, $L(\boldsymbol{\theta} \mid \boldsymbol{y}, \boldsymbol{z}) \equiv p(\boldsymbol{y} \mid \boldsymbol{z}, \boldsymbol{\theta}, \boldsymbol{\beta}) p(\boldsymbol{z} \mid \boldsymbol{\theta}, \boldsymbol{\beta})$, está intrisicamente ligada ao método da composição. Pois, empíricamente não haverá diferença alguma entre realizar inferência a partir da verossimilhança original que está associada a um experimento simples ou a partir da verossimilhança de dados aumentados que está associada a um experimento sequencial. É interessante notar ainda que uma das grandes vantagens de se trabalhar com a verossimilhança aumentada deve-se à possibilidade de se obter expressões simplificadas para o processo de estimação. Note também que idéia análoga é utilizada para a construção do algoritmo EM ("Expected and Maximization") em que o objetivo é obter estimativas de máxima verossimilhança na presença de dados omissos, faltantes e/ou latentes (Dempster et al., 1977; Bock; Aitkin, 1981).

No que segue, apresentamos uma proposição que estabelece um modelo de dados aumentado para o modelo Kd3PP. Esta proposição representa uma extensão direta para o caso multidimensional daquela proposta por Sahu (2002) que considerou o caso unidimensional. Béguin e Glas (2001) também propuseram tal extensão, no entanto, a que apresentamos em seguida é ligeiramente diferente porque a introdução de variáveis latentes associadas aos parâmetros de acerto casual é feita segundo a proposta de Sahu (2002).

Matos, G. S.

IME/USP 


\subsection{Estimação Bayesiana do Modelo Kd3PP-tA (e Km3PP) via Método} DAGS

Proposição 4.2.1. Seja $P\left(Y_{i j}=y_{i j} \mid \beta_{1 j}, \boldsymbol{\beta}_{2 j}, \beta_{3 j}, \boldsymbol{\theta}_{i}\right)=\pi_{i j}^{y_{i j}}\left[1-\pi_{i j}\right]^{1-y_{i j}}, y_{i j}=0,1$, com $\pi_{i j}=\beta_{3 j}+\left(1-\beta_{3 j}\right) \Phi\left(\eta_{i j}\right)$, o modelo K-dimensional de 3 parâmetros probito da TRI; denotado por Kd3PP; onde $\beta_{3 j} \in(0,1)$, $\Phi($.$) é a f.d.a. da normal padrão e \eta_{i j}=-\beta_{1 j}+$ $\boldsymbol{\theta}_{i}^{\prime} \boldsymbol{\beta}_{2 j}$ com $\boldsymbol{\theta}_{i}, \boldsymbol{\beta}_{2 j} \in \mathbb{R}^{K}$ e $\beta_{1 j} \in \mathbb{R}$. Segue que um modelo de dados aumentados associado ao modelo Kd3PP é dado por

$P\left(Y_{i j}=y_{i j}, U_{i j}=u_{i j}, v_{i j} \mid \boldsymbol{\theta}_{i}, \beta_{1 j}, \boldsymbol{\beta}_{2 j}, \beta_{3 j}\right)=\beta_{3 j}^{u_{i j}}\left(1-\beta_{3 j}\right)^{1-u_{i j}} \phi\left(v_{i j} \mid \eta_{i j}, 1\right) \boldsymbol{I}\left(y_{i j}, u_{i j}, v_{i j}\right)$,

em que:

- $u_{i j} \sim \operatorname{Bernolli}\left(\beta_{3 j}\right)$

- $\phi\left(v_{i j} \mid \eta_{i j}, 1\right)$ é a f.d.p. da variável $v_{i j}$ cuja distribuição é normal com média $\eta_{i j}=\boldsymbol{\Theta}_{i}^{\prime} \boldsymbol{\beta}_{12 j}=-\beta_{1 j}+\boldsymbol{\theta}_{i}^{\prime} \boldsymbol{\beta}_{2 j}$ e variância $1 ;$ e

- $\boldsymbol{I}\left(y_{i j}, u_{i j}, v_{i j}\right)=I\left(y_{i j}=0\right) I\left(u_{i j}=0\right) I\left(v_{i j} \leq 0\right)$

$+I\left(y_{i j}=1\right) I\left(u_{i j}=0\right) I\left(v_{i j}>0\right)$

$+I\left(y_{i j}=1\right) I\left(u_{i j}=1\right) I\left(v_{i j} \leq 0\right)$

$+I\left(y_{i j}=1\right) I\left(u_{i j}=1\right) I\left(v_{i j}>0\right)$

é a usual função indicadora que assume o valor 1 ou 0 , conforme o valor assumido por $\left(y_{i j}, u_{i j}, v_{i j}\right)$.

Prova: Com base na expressão (4.1), observe que

$$
\begin{aligned}
P\left(Y_{i j}=y_{i j} \mid \beta_{1 j}, \boldsymbol{\beta}_{2 j}, \beta_{3 j}, \boldsymbol{\theta}_{i}\right) & =\sum_{u=0}^{1} \int_{-\infty}^{\infty} \beta_{3 j}^{u_{i j}}\left(1-\beta_{3 j}\right)^{1-u_{i j}} \phi\left(v_{i j} \mid \eta_{i j}, 1\right) \boldsymbol{I}\left(y_{i j}, u_{i j}, v_{i j}\right) d v_{i j} \\
& =\left(1-\beta_{3 j}\right)\left[1-\Phi\left(\eta_{i j}\right)\right] I\left(y_{i j}=0\right)+\left(1-\beta_{3 j}\right) \Phi\left(\eta_{i j}\right) I\left(y_{i j}=1\right) \\
& +\beta_{3 j} I\left(y_{i j}=1\right),
\end{aligned}
$$

de modo que

$$
P\left(Y_{i j}=1 \mid \beta_{1 j}, \boldsymbol{\beta}_{2 j}, \beta_{3 j}, \boldsymbol{\theta}_{i}\right)=\pi_{i j} \text { e } P\left(Y_{i j}=0 \mid \beta_{1 j}, \boldsymbol{\beta}_{2 j}, \beta_{3 j}, \boldsymbol{\theta}_{i}\right)=1-\pi_{i j} .
$$

Segue, portanto, que

$$
P\left(Y_{i j}=y_{i j}, U_{i j}=u_{i j}, v_{i j} \mid \boldsymbol{\theta}_{i}, \beta_{1 j}, \boldsymbol{\beta}_{2 j}, \beta_{3 j}\right)=\beta_{3 j}^{u_{i j}}\left(1-\beta_{3 j}\right)^{1-u_{i j}} \phi\left(v_{i j} \mid \eta_{i j}, 1\right) \boldsymbol{I}\left(y_{i j}, u_{i j}, v_{i j}\right)
$$

é um modelo de dados aumentados associado ao Kd3PP.

Após obter um modelo de dados aumentados equivalente ao modelo original Kd3PP, o passo seguinte para a obtenção de uma verossimilhança aumentada é acrescentar aos dados observados, $\boldsymbol{y}$, as variáveis latentes auxiliares $\boldsymbol{u}=\left(u_{11}, \ldots, u_{i j}, \ldots, u_{N J}\right)^{\prime}$ e $\boldsymbol{v}=$ $\left(v_{11}, \ldots, v_{i j}, \ldots, v_{N J}\right)^{\prime}$, conforme apresentamos na seguinte proposição. 
Proposição 4.2.2 (Verossimilhança Aumentada do Modelo Kd3PP). Seja y o vetor de dados observados segundo o modelo Kd3PP da TRI. Se $\boldsymbol{u}=\left(u_{11}, \ldots, u_{i j}, \ldots, u_{N J}\right)^{\prime}$ e $\boldsymbol{v}=\left(v_{11}, \ldots, v_{i j}, \ldots, v_{N J}\right)^{\prime}$ são os vetores de variáveis latentes auxiliares descritas na Proposição 4.2.1, segue que uma verossimilhança de dados aumentados associada ao modelo Kd3PP é dada por

$$
L\left(\boldsymbol{\theta}, \boldsymbol{\beta} \mid \boldsymbol{D}_{a}\right)=\prod_{i=1}^{N} \prod_{j=1}^{J}\left\{\beta_{3 j}^{u_{i j}}\left(1-\beta_{3 j}\right)^{1-u_{i j}} \phi\left(v_{i j} \mid \eta_{i j}, 1\right) \boldsymbol{I}\left(y_{i j}, u_{i j}, v_{i j}\right)\right\}^{m_{i j}}
$$

em que:

- $\boldsymbol{D}_{a}=\left(\boldsymbol{y}^{\prime}, \boldsymbol{u}^{\prime}, \boldsymbol{v}^{\prime}, \boldsymbol{M}\right)^{\prime}$ representa o vetor de dados aumentados;

- $\boldsymbol{M}=\left[m_{i j}\right]$ é uma matriz $N \times J$ onde as componentes $m_{i j}$ são variáveis observadas que assumem o valor 1 (ou 0) se o item $i$ é submetido (ou não) ao j-ésimo respondente, respectivamente.

Na próxima sub-seção apresentamos as especificações das distribuições a priori associadas aos vários parâmetros de interesse do modelo Kd3PP.

\subsubsection{Distribuição t-Assimétrica para os Traços Latentes e Espe- cificação de Distribuições a Priori para os Parâmetros dos Itens}

De acordo com a discussão apresentada na introdução deste capítulo, a distribuição a ser adotada como priori para os traços latentes será a t-Assimétrica multivariada proposta por Sahu et al. (2003). No que segue, $S T_{\nu}\left(\boldsymbol{\mu}_{\theta}, \boldsymbol{\Sigma}_{\theta}, \boldsymbol{D}\right)$ denotará uma distribuição t-Assimétrica com parâmetros $\boldsymbol{\mu}_{\theta}, \boldsymbol{\Sigma}_{\theta}, \boldsymbol{D}$ e $\nu$ correspondentes à localização, escala, assimetria e graus de liberdade, respectivamente. O parâmetro $\boldsymbol{\mu}_{\theta}$ é um vetor de dimensão $K, \boldsymbol{\Sigma}_{\theta}$ é uma matriz positiva definida de dimensão $K, \boldsymbol{D}=\operatorname{diag}(\boldsymbol{\delta})$ é uma matriz diagonal de ordem $K, \boldsymbol{\delta}=\left(\delta_{1}, \ldots, \delta_{K}\right)^{\prime}$ e $\nu$ é um escalar.

Dentre outras considerações, a escolha da distribuição proposta por Sahu et al. (2003) deve-se a possibilidade da mesma admitir uma representação hierárquica, o que simplifica de modo considerável a implementação de algoritmos do tipo MCMC, em particular o Gibbs. Neste sentido, se $\boldsymbol{\theta}_{1}, \ldots, \boldsymbol{\theta}_{N}$ são vetores de variáveis aleatórias independentes tais que $\boldsymbol{\theta}_{i} \mid \boldsymbol{\eta}_{i} \equiv \boldsymbol{\eta} \sim S T_{\nu}(\boldsymbol{\eta}) \quad i=1, \ldots, N$, com $\boldsymbol{\eta}=\left(\boldsymbol{\mu}_{\theta}, \boldsymbol{\Sigma}_{\theta}, \boldsymbol{D}\right)$, então a seguinte 


\subsection{Estimação Bayesiana do Modelo Kd3PP-tA (e Km3PP) via Método} DAGS

representação hierárquica é válida

$$
\begin{array}{ll}
\boldsymbol{\theta}_{i} \mid \boldsymbol{z}_{i}, \boldsymbol{\Sigma}_{\theta}, \boldsymbol{\mu}_{\theta}, \boldsymbol{D}, \omega_{i} & \sim N_{K}\left(\boldsymbol{\mu}_{\theta}+\boldsymbol{D} \boldsymbol{Z}_{i}, \frac{\boldsymbol{\Sigma}_{\theta}}{\omega_{i}}\right) \\
\boldsymbol{Z}_{i} & \sim N_{K}(\mathbf{0}, \boldsymbol{I}) I\left(\boldsymbol{z}_{i}>\mathbf{0}\right) \\
\boldsymbol{Q}=\boldsymbol{\Sigma}_{\theta}^{-1} & \sim W_{K}\left(2 r, 2 \boldsymbol{\Sigma}_{Q}\right) \\
\boldsymbol{\mu}_{\theta} & \sim N_{K}\left(\boldsymbol{\mu}_{0}, \boldsymbol{\Lambda}_{0}\right) \\
\boldsymbol{\delta} & \sim N_{K}\left(\mathbf{0}, \boldsymbol{\Gamma}_{0}\right) \\
\omega_{i} & \sim \operatorname{Gama}(\nu / 2, \nu / 2) \\
\nu & \sim \operatorname{Gama}(1,0.1) I(\nu>2),
\end{array}
$$

em que:

- $\boldsymbol{\mu}_{\theta}, \boldsymbol{\Sigma}_{\theta}, \boldsymbol{D}=\operatorname{diag}(\boldsymbol{\delta})$ e $\nu$ são os hiperparâmetros da distribuição t-Assimétrica;

- $W_{K}\left(2 r, 2 \boldsymbol{\Sigma}_{Q}\right)$ denota a distribuição Wishart com graus de liberdade $2 r(2 r \geq K)$ e matriz de parâmetros de escala $2 \Sigma_{Q}$, simétrica e positiva definida (ver descrição da parametrização adotada logo em seguida).

- $\boldsymbol{\mu}_{0}$ é um vetor pré-determinado de médias e $\boldsymbol{\Lambda}_{0}$ matrix de variâncias-covariâncias que pode ser especificada a partir de uma matriz de precisão $\boldsymbol{\Lambda}_{0}^{-1}$. Por exemplo, $\boldsymbol{\Lambda}_{0}^{-1}=\mathbf{0}_{K}$ possibilita expressar uma informação vaga (distribuição a priori uniforme e imprópria) sobre o vetor de médias populacionais dos traços latentes $\boldsymbol{\mu}_{\theta}$. Por outro lado, $\boldsymbol{\Lambda}_{0}^{-1}=\lambda_{0} \boldsymbol{I}_{K}$ com valor $\lambda_{0}$ assumindo um valor suficientemente grande possibilita expressar uma opinião altamente precisa sobre o vetor de médias $\boldsymbol{\mu}_{\theta}$.

- $\Gamma_{0}$ é uma matriz positiva definida pré-especificada que também pode ser expressa em termos de uma matriz de precisão.

- $\operatorname{Gama}(\alpha, \beta)$ denota a distribuição Gama com valor esperado $\alpha \beta$ e variância $\alpha \beta^{2}$, conforme parametrização a ser descrita abaixo, $\operatorname{com} K=1$.

Os parâmetros da distribuição da matriz de precisão $\boldsymbol{Q}=\boldsymbol{\Sigma}_{\theta}^{-1}$ devem ser previamente fixados segundo a notação e parametrização apresentada por Gelman et al. (1995, pag. $475)$. Neste caso, se $\boldsymbol{Q} \sim W_{K}(\alpha, \boldsymbol{\beta})$ com densidade proporcional a

$$
|\boldsymbol{\beta}|^{-\alpha / 2}|\boldsymbol{Q}|^{\frac{1}{2}(\alpha-K-1)} e^{-\frac{1}{2} \operatorname{tr}\left(\boldsymbol{\beta}^{-1} \boldsymbol{Q}\right)},
$$

em que $\operatorname{tr}\left(\boldsymbol{\beta}^{-1} \boldsymbol{Q}\right)$ denota o traço da matriz $\boldsymbol{\beta}^{-1} \boldsymbol{Q} \operatorname{com} \boldsymbol{Q}$ e $\boldsymbol{\beta}$ matrizes simétricas e positivas definidas, então $E(\boldsymbol{Q})=\alpha \boldsymbol{\beta}$. Outra informação possivelmente útil para a especificação da distribuição a priori segue do fato de que $\boldsymbol{\Sigma}_{\theta}=\boldsymbol{Q}^{-1} \sim$ Wishart-Invertida $_{K}\left(\alpha, \boldsymbol{\beta}^{-1}\right)$ e

Matos, G. S.

IME/USP 
$E\left(\boldsymbol{\Sigma}_{\theta}\right)=\frac{1}{\alpha-K-1} \boldsymbol{\beta}$, desde que a função densidade correspondente seja proporcionalmente dada por

$$
|\boldsymbol{\beta}|^{\alpha / 2}|\boldsymbol{\Sigma}|^{-\frac{1}{2}(\alpha+K+1)} e^{-\frac{1}{2} \operatorname{tr}\left(\boldsymbol{\beta} \boldsymbol{\Sigma}^{-1}\right)} .
$$

É importante dizer que a parametrização da distribuição Wishart adotada por Sahu et al. (2003) difere da nossa apenas por não considerarem no núcleo da densidade em (4.4) a inversa da matriz $\boldsymbol{\beta}$. É importante dizer, ainda, que a escolha sobre a particular parametrização descrita em Gelman et al. (1995, pag. 475) deve-se a uma recomendação encontrada no manual de desenvolvedores ${ }^{\dagger}$ de funções para o pacote MCMCpack (Martin; Quinn, 2006) do ambiente computacional e estatístico $\mathrm{R}$. Ainda com relação à especificação da distribuição a priori Wishart lembramos que uma distribuição não-informativa é obtida quando $\alpha \rightarrow 0$.

De acordo com Sahu et al. (2003), para obter a distribução normal multivariada assimétrica a partir da representação hierárquica (4.3) é suficiente omitir as duas últimas distribuições especificadas, além de considerar $\omega_{i}=1, \quad \forall i$. Outro aspecto importante a ser destacado na especificação da distribuição a priori para os traços latentes no presente trabalho, refere-se a independência assumida entre os hiperparâmetros $\boldsymbol{\mu}_{\theta}$ e $\boldsymbol{\Sigma}_{\theta}$ pois esta é mais uma característica que difere do trabalho de Béguin e Glas (2001) que assumiram dependência entre estes hiperparâmetros quando adotaram uma distribuição conjunta normal-Wishart invertida.

Como já foi dito, as distribuições $t$ e normal multivariada assimétrica propostas por Sahu et al. (2003) apresentam propriedades de bastante interesse teórico e prático. Uma delas diz que não existe correlação entre as componentes do vetor aleatório quando a matriz de escala $\boldsymbol{\Sigma}_{\theta}$ é diagonal, ou seja, $\boldsymbol{\Sigma}_{\theta}=\operatorname{diag}\left(\sigma_{1}, \ldots, \sigma_{K}\right)$. No caso particular da normal multivariada assimétrica isto corresponde a uma relação de independência entre as variáveis aleatórias marginais. Além disso, a recíproca também é verdadeira; assim como é no caso da normal multivariada simétrica. Outra característica também de bastante interesse consiste da possibilidade de se ter como caso particular as distribuições t e normal simétricas. Isto de fato ocorre quando o parâmetro associado à forma, ou melhor, à assimetria é nulo, isto é, $\boldsymbol{\delta}=\mathbf{0}$.

Para completar as especificações necessárias à inferência bayesiana sobre os parâmetros do modelo Kd3PP, resta-nos definir as distribuições a priori dos parâmetros dos itens. Neste caso, assim como em vários trabalhos encontrados na literatura, assumiremos distribuições normais independentes para as distribuições a priori dos parâmetros $\beta_{1 j}$ e $\boldsymbol{\beta}_{2 j}$ e, para o parâmetro de acerto casual uma distribuição beta, ou seja:

$$
\begin{aligned}
\boldsymbol{\beta}_{12 j} \equiv\left(\beta_{1 j}, \boldsymbol{\beta}_{2 j}^{\prime}\right)^{\prime} & \sim N_{1+K}\left(\boldsymbol{b}_{0, j}, \boldsymbol{B}_{0, j}\right), j=1, \ldots, J \\
\beta_{3 j} & \sim \operatorname{Beta}\left(a_{j}, b_{j}\right),
\end{aligned}
$$

$\dagger$ Disponível em: http://mcmcpack.wustl.edu/wiki/index.php/MCMCpack_Development_Page.

Matos, G. S.

IME/USP 


\subsection{Estimação Bayesiana do Modelo Kd3PP-tA (e Km3PP) via Método} DAGS

em que $\boldsymbol{b}_{0, j}=\left(\mu_{\beta_{1} j}, \mu_{\beta_{2} j 1}, \ldots, \mu_{\beta_{2} j K}\right)^{\prime}$ é um vetor de médias e $\boldsymbol{B}_{0, j}=\operatorname{diag}\left(\sigma_{\beta_{1} j}^{2}, \sigma_{\beta_{2} j 1}^{2}, \ldots, \sigma_{\beta_{2} j K}^{2}\right)$ é uma matriz de covariância diagonal.

Lembramos que distribuições não-informativas (vagas) são obtidas adotando-se variâncias grandes na distribuição normal ou de modo equivalente (e mais eficaz) adotando-se parâmetros de precisão (inverso da variância) nulos. No caso da distribuição Beta, a distribuição a priori não-informativa é obtida quando $a_{j}=b_{j}=1$, por exemplo. Lembramos, ainda, que distribuições a priori normais truncadas para os parâmetros de discriminação podem ser facilmente consideradas e isto não representa compromentimento algum sobre o que iremos desenvolver nas próximas sub-seções.

\subsubsection{Densidade a Posteriori Aumentada}

Usando a verossimilhança aumentada do modelo Kd3PP da Proposição $4.2 .2 \operatorname{com}(\boldsymbol{U}, \boldsymbol{V})$ representando a coleção de variáveis latentes auxiliares e sob independência entre as distribuições a priori conjuntas (4.3) e (4.5), a densidade a posteriori aumentada associada ao vetor $\boldsymbol{\zeta}=\left(\boldsymbol{\theta}, \boldsymbol{\beta}, \boldsymbol{U}, \boldsymbol{V}, \boldsymbol{Z}, \boldsymbol{\mu}_{\theta}, \boldsymbol{Q}, \boldsymbol{D}, \boldsymbol{\omega}, \nu\right) \operatorname{com} \boldsymbol{Q}=\boldsymbol{\Sigma}_{\theta}^{-1}, \boldsymbol{D}=\operatorname{diag}(\boldsymbol{\delta})$ em que $\boldsymbol{\delta}=$ $\left(\delta_{1}, \ldots, \delta_{K}\right)^{\prime}$ e $\boldsymbol{\omega}=\left(\omega_{1}, \ldots, \omega_{N}\right)^{\prime}$, é tal que

$$
\begin{aligned}
\pi(\boldsymbol{\zeta} \mid \boldsymbol{y}, \boldsymbol{M}) & \propto \pi(\boldsymbol{y}, \boldsymbol{U}, \boldsymbol{V}, \boldsymbol{M} \mid \boldsymbol{\theta}, \boldsymbol{\beta}) \pi\left(\boldsymbol{\theta} \mid \boldsymbol{z}, \boldsymbol{\Sigma}_{\theta}, \boldsymbol{\mu}_{\theta}, \boldsymbol{D}, \boldsymbol{\omega}\right) \pi(\boldsymbol{z}) \pi\left(\boldsymbol{\Sigma}_{\theta}\right) \pi\left(\boldsymbol{\mu}_{\theta}\right) \pi(\boldsymbol{\delta}) \pi(\boldsymbol{\omega}) \pi(\nu) \\
& \times \pi\left(\boldsymbol{\beta}_{12}\right) \pi\left(\boldsymbol{\beta}_{3}\right) \\
& \propto \prod_{i=1}^{N} \prod_{j=1}^{J}\left\{\beta_{3 j}^{u_{i j}}\left(1-\beta_{3 j}\right)^{1-u_{i j}} \exp \left\{-\frac{1}{2}\left(v_{i j}-\boldsymbol{\Theta}_{i}^{\prime} \boldsymbol{\beta}_{12 j}\right)^{2}\right\} \boldsymbol{I}\left(y_{i j}, u_{i j}, v_{i j}\right)\right\} \\
& \times \prod_{i=1}^{N}\left|\frac{\boldsymbol{\Sigma}_{\theta}}{\omega_{i}}\right|^{-1 / 2} \exp \left\{-\frac{1}{2}\left(\boldsymbol{\theta}_{i}-\boldsymbol{\mu}_{\theta}-\boldsymbol{D} \boldsymbol{z}_{i}\right)^{\prime}\left(\frac{\boldsymbol{\Sigma}_{\theta}}{\omega_{i}}\right)^{-1}\left(\boldsymbol{\theta}_{i}-\boldsymbol{\mu}_{\theta}-\boldsymbol{D} \boldsymbol{z}_{i}\right)\right\} \\
& \times \prod_{i=1}^{N} \exp \left\{-\frac{1}{2} \boldsymbol{z}_{i}^{\prime} \boldsymbol{z}_{i}\right\} I\left(\boldsymbol{z}_{i}>\mathbf{0}\right) \\
& \times|\boldsymbol{Q}|^{\frac{1}{2}(2 r-K-1)} \exp \left\{-\frac{1}{2} t r\left(\frac{1}{2} \boldsymbol{\Sigma}_{Q}^{-1} \boldsymbol{Q}\right)\right\} \times \exp \left\{-\frac{1}{2}\left(\boldsymbol{\mu}_{\theta}-\boldsymbol{\mu}_{0}\right)^{\prime} \boldsymbol{\Lambda}_{0}^{-1}\left(\boldsymbol{\mu}_{\theta}-\boldsymbol{\mu}_{0}\right)\right\} \\
& \times \exp \left\{-\frac{1}{2} \boldsymbol{\delta}^{\prime} \boldsymbol{\Gamma}_{0}^{-1} \boldsymbol{\delta}\right\} \times\left\{\prod_{i=1}^{N} \frac{1}{\Gamma\left(\frac{\nu}{2}\right)}\left(\frac{2}{\nu}\right)^{\frac{\nu / 2}{2}} \omega_{i}^{\frac{\nu / 2}{2}-1} \exp \left\{-\frac{1}{2}\left(\frac{2}{\nu}\right) \omega_{i}\right\} I\left(\omega_{i}>0\right)\right\} \\
& \times \exp \left\{-\frac{1}{2} 10 \nu\right\} I(\nu>2) \\
& \times \prod_{j=1}^{J} \exp \left\{-\frac{1}{2}\left(\boldsymbol{\beta}_{12 j}-\boldsymbol{b}_{0, j}\right)^{\prime} \boldsymbol{B}_{0, j}^{-1}\left(\boldsymbol{\beta}_{12 j}-\boldsymbol{b}_{0, j}\right)\left(\beta_{3 j}\right)^{a_{j}-1}\left(1-\beta_{3 j}\right)^{b_{j}-1} .\right.
\end{aligned}
$$

Matos, G. S. 


\subsection{Estimação Bayesiana do Modelo Kd3PP-tA (e Km3PP) via Método DAGS}

Como podemos perceber, a obtenção direta de estimativas a partir desta distribuição é algo intratável. Entretanto, as distribuições condicionais completas necessárias à implementação de um algoritmo Gibbs (Casella; George, 1992) são de fácil obtenção. No Apêndice A apresentamos detalhes sobre como obter estas distribuições e na seção seguinte apresentamos os passos necessários à implementação computacional do método DAGS.

\subsubsection{Implementação do Algoritmo DAGS}

Neste momento, apresentamos uma versão básica do algoritmo DAGS a qual permite obter amostras marginais dos parâmetros do modelo Kd3PP-tA. Dizemos versão básica pelo fato do algoritmo não considerar a introdução de qualquer restrição paramétrica, necessária, por exemplo, para contornar possíveis problemas de não-identificabilidade (Seção 3.3).

É importante destacar que por simplicidade, a notação do tipo $\boldsymbol{\tau} \mid \ldots, \boldsymbol{y}$ representará neste trabalho a distribuição condicional a posteriori de um determinado parâmetro $\tau$ qualquer condicionado à todos os demais parâmetros $\zeta$ com exceção do parâmetro de interesse $\boldsymbol{\tau}$. O caso em que o vetor de respostas $\boldsymbol{y}$ não aparece na notação corresponde a uma distribuição condicional completa de interesse que não depende diretamente dos dados (respostas aos itens) ou de qualquer subconjunto dos dados.

Para implementar a versão básica do algoritmo DAGS os seguintes passos devem ser executados:

- Passo 1: Fazer $h=0$ e adotar valores iniciais para as diversas quantidades nãoobserváveis, ou seja, para $\boldsymbol{u}, \boldsymbol{v}, \boldsymbol{\theta}_{i}, \forall i, \boldsymbol{\beta}_{12 j}, \beta_{3 j}, \forall j, \boldsymbol{z}, \boldsymbol{Q}=\boldsymbol{\Sigma}_{\theta}^{-1}, \boldsymbol{\mu}_{\theta}$,

$\boldsymbol{D}=\operatorname{diag}(\boldsymbol{\delta}), \boldsymbol{\omega}$ e $\nu$.

- Passo 2: Fazer o incremento $h=h+1$.

- Passo 3:

- Dado $m_{i j}=1$, isto é, que o $j$-ésimo item foi apresentado ao $i$-ésimo indivíduo e que a resposta $y_{i j}$ foi observada, gerar amostras independentes $u_{i j}^{(h)}$ e $v_{i j}^{(h)}$ da seguinte forma:

* Se $y_{i j}=0$, então $u_{i j}^{(h)}=0$ e $v_{i j}^{(h)}=v_{i j}^{*}$ em que $v_{i j}^{*}$ é um valor simulado de uma distribuição $N\left(\eta_{i j}^{(h-1)}, 1\right) I\left(v_{i j}<0\right)$.

* Quando $y_{i j}=1$, considere o seguinte:

- Se $u_{i j}^{(h-1)}=0$, então $v_{i j}^{(h)}=v_{i j}^{*}$ em que $v_{i j}^{*}$ é simulado de uma distribuição $N\left(\eta_{i j}^{(h-1)}, 1\right) I\left(v_{i j}>0\right)$;

Matos, G. S.

IME/USP 


\subsection{Estimação Bayesiana do Modelo Kd3PP-tA (e Km3PP) via Método} DAGS

- Caso contrário, i.e., se $u_{i j}^{(h-1)}=1$, então $v_{i j}^{(h)}=v_{i j}^{*}$ em que $v_{i j}^{*} \sim N\left(\eta_{i j}^{(h-1)}, 1\right)$ e, neste caso:

- Se $v_{i j}^{(h)}<0$, fazer $u_{i j}^{(h)}=1$, senão fazer $u_{i j}^{(h)}=u_{i j}^{*}$, em que $u_{i j}^{*} \sim$ $\operatorname{Ber}\left(\beta_{3 j}\right)$.

- Caso $m_{i j}=1$, porém $y_{i j}$ não tenha sido observada por motivos completamente ao acaso (veja discussão na página 8), o seguinte procedimento de imputação, por exemplo, pode ser adotado:

* Considerar $v_{i j}^{(h)}=v_{i j}^{*} \sim N\left(\eta_{i j}^{(h-1)}, 1\right)$ e:

- Caso $v_{i j}^{(h)}<0$, fazer $u_{i j}^{(h)}=1$, senão faça $u_{i j}^{(h)}=u_{i j}^{*}$, onde $u_{i j}^{*} \sim \operatorname{Ber}\left(\beta_{3 j}\right)$.

- Passo 4: Para $i=1, \ldots, N$ gerar amostra de

$$
\boldsymbol{\theta}_{i} \mid \ldots \sim N_{K}\left(\boldsymbol{F}_{i} \boldsymbol{f}_{i}, \boldsymbol{F}_{i}\right)
$$

com

$$
\begin{aligned}
& -\boldsymbol{F}_{i}=\left(\omega_{i} \boldsymbol{Q}+\boldsymbol{\beta}_{2 .}^{(i)^{\prime}} \frac{1}{\sigma^{2}} \boldsymbol{\beta}_{2 .}^{(i)}\right)^{-1} \mathrm{e} \\
& -\boldsymbol{f}_{i}=\omega_{i} \boldsymbol{Q}\left(\boldsymbol{\mu}_{\theta}+\boldsymbol{D} \boldsymbol{Z}_{i}\right)+\boldsymbol{\beta}_{2 .}^{(i)^{\prime}} \frac{1}{\sigma^{2}} \boldsymbol{v}_{i}^{*},
\end{aligned}
$$

onde $\sigma^{2}=1, \quad \boldsymbol{\beta}_{2}^{(i)}$ é uma matriz $n_{i} \times K$ cuja $j$-ésima linha corresponde aos parâmetros de discrimação $\boldsymbol{\beta}_{2 j}^{\prime}=\left(\boldsymbol{\beta}_{21}^{(h)}, \ldots, \boldsymbol{\beta}_{2 k}^{(h)}, \ldots, \boldsymbol{\beta}_{2 K}^{(h)}\right)$ do $j$-ésimo item submetido ao $i$-ésimo respondente, $n_{i}=\operatorname{card}\left\{\boldsymbol{J}_{i}\right\}$ é a cardinalidade de $\boldsymbol{J}_{i}$ que representa o conjunto dos índices dos itens submetidos ao $i$-ésimo respondente e $\boldsymbol{v}_{i}^{*}=\left(v_{i 1}^{*}, \ldots, v_{i j}^{*}, \ldots, v_{i n_{i}}^{*}\right)^{\prime}, \operatorname{com} v_{i j}^{*}=v_{i j}^{(h)}+\beta_{1 j}^{(h)}, \quad j \in \boldsymbol{J}_{i}$.

- Passo 5: Para $j=1, \ldots J$, gerar amostra da seguinte distribuição condicional completa

$$
\boldsymbol{\beta}_{12 j} \mid \ldots \sim N_{1+K}\left(\boldsymbol{\varphi}_{j} \boldsymbol{m}_{j}, \boldsymbol{\varphi}_{j}\right)
$$

em que $\boldsymbol{\varphi}_{j}=\left(\boldsymbol{B}_{0, j}^{-1}+\boldsymbol{\Theta}^{(j)^{\prime}} \boldsymbol{\Theta}^{(j)}\right)^{-1}$ e $\quad \boldsymbol{m}_{j}=\left(\boldsymbol{B}_{0, j}^{-1} \boldsymbol{b}_{0, j}+\boldsymbol{\Theta}^{(j)^{\prime}} \boldsymbol{v}_{j}\right)$ com

- $\boldsymbol{\Theta}^{(j)}=\left[-\mathbf{1}, \boldsymbol{\theta}^{(j)}\right]$ sendo uma matriz $n_{j} \times(1+K)$ em que:

* $n_{j}=\operatorname{card}\left\{\boldsymbol{I}_{j}\right\}$ representa a cardinalidade do conjunto $\boldsymbol{I}_{j}$ que por sua vez representa o conjunto dos índices dos respondentes que foram apresentados ao $j$-ésimo item.

Matos, G. S.

IME/USP 


\subsection{Estimação Bayesiana do Modelo Kd3PP-tA (e Km3PP) via Método}

* 1 é um vetor unitário $n_{j} \times 1$ e $\boldsymbol{\theta}^{(j)}$ é uma matriz $n_{j} \times K$ cujas linhas são os vetores de traços latentes $\left(\boldsymbol{\theta}_{i}^{\prime}\right)^{(h)}, \quad i \in \boldsymbol{I}_{j}$.

- $\boldsymbol{B}_{0, j}$ e $\boldsymbol{b}_{0, j}$ são os hiperparâmetros de $\beta_{1 j}$ e $\boldsymbol{\beta}_{2 j}$, tal como descrito em (4.5) e

$-\boldsymbol{v}_{j}=\left(v_{1 j}^{(h)}, \ldots, v_{i j}^{(h)}, \ldots, v_{n_{j}}^{(h)}\right)^{\prime}, \quad i \in \boldsymbol{I}_{j}$.

- Passo 6: Gerar amostra para todo $j=1, \ldots, J$ da seguinte distribuição condicional completa

$$
\beta_{3 j} \mid \ldots \sim \operatorname{Beta}\left(u_{. j}+a_{j}, n_{j}-u_{. j}+b_{j}\right)
$$

em que

$$
-u_{. j}=\sum_{i \in \boldsymbol{I}_{j}} u_{i j}^{(h)}, \mathrm{com}
$$

* $\boldsymbol{I}_{j}$ representando o conjunto dos índices dos respondentes que foram apresentados ao $j$-ésimo item, e

$* n_{j}=\operatorname{card}\left\{\boldsymbol{I}_{j}\right\}$ é a cardinalidade do conjunto $\boldsymbol{I}_{j}$.

- $a_{j}$ e $b_{j}$ são os hiperparâmetros da distribuição de $\beta_{3 j}$.

- Passo 7: Gerar amostras aleatórias independentes $\boldsymbol{z}_{1}, \ldots, \boldsymbol{z}_{N}$ a partir de distribuições

$$
\boldsymbol{Z}_{i} \mid \ldots \stackrel{\text { ind. }}{\sim} N_{K}\left(\boldsymbol{A}_{i}^{-1} \boldsymbol{a}_{i}, \boldsymbol{A}_{i}^{-1}\right) I\left(\boldsymbol{z}_{i}>\mathbf{0}\right)
$$

em que

$$
\boldsymbol{A}_{i}=\omega_{i}^{(h-1)} \boldsymbol{D}^{(h-1)} \boldsymbol{Q}^{(h)} \boldsymbol{D}^{(h-1)}+\boldsymbol{I} \quad \text { e } \quad \boldsymbol{a}_{i}=\omega_{i}^{(h-1)} \boldsymbol{D}^{(h-1)} \boldsymbol{Q}^{(h)}\left(\boldsymbol{\theta}_{i}^{(h-1)}-\boldsymbol{\mu}_{\theta}^{(h)}\right) .
$$

- Passo 8: Obter amostra $\boldsymbol{Q}^{(h)}$ da distribuição condicional completa dada por

$$
\boldsymbol{Q} \mid \ldots \sim W_{K}(2 r+N, \boldsymbol{S}),
$$

em que

$$
\begin{aligned}
\boldsymbol{S} & =\left\{\sum_{i=1}^{N} \omega_{i}^{(h-1)}\left(\boldsymbol{\theta}_{i}^{(h-1)}-\boldsymbol{\mu}_{\theta}^{(h-1)}-\boldsymbol{D}^{(h-1)} \boldsymbol{z}_{i}^{(h-1)}\right)\left(\boldsymbol{\theta}_{i}^{(h-1)}-\boldsymbol{\mu}_{\theta}^{(h-1)}-\boldsymbol{D}^{(h-1)} \boldsymbol{z}_{i}^{(h-1)}\right)^{\prime}\right. \\
& \left.+\frac{1}{2} \boldsymbol{\Sigma}_{Q}^{-1}\right\}^{-1} .
\end{aligned}
$$

- Passo 9: Obter amostra $\boldsymbol{\mu}_{\theta}^{(h)}$ cuja distribuição condicional completa é dada por

$$
\boldsymbol{\mu}_{\theta} \mid \ldots \sim N_{K}\left(\boldsymbol{W}^{-1} \boldsymbol{b}, \boldsymbol{W}^{-1}\right)
$$

em que

Matos, G. S. 


$$
\begin{aligned}
-\boldsymbol{W} & =N \boldsymbol{Q}^{(h)} \bar{\omega}+\boldsymbol{\Lambda}_{0}^{-1} \quad \operatorname{com} \boldsymbol{Q}^{(h)}=\left(\boldsymbol{\Sigma}_{\theta}^{-1}\right)^{(h)} \\
-\boldsymbol{b} & =N \boldsymbol{Q}^{(h)} \overline{\boldsymbol{E}}+\boldsymbol{\Lambda}_{0}^{-1} \boldsymbol{\mu}_{0} \quad \text { com } \\
& * \bar{\omega}=\frac{\sum_{i=1}^{N} \omega_{i}^{(h-1)}}{N} \text { e } \\
& * \overline{\boldsymbol{E}}=\frac{\sum_{i=1}^{N} \boldsymbol{C}_{i} \omega_{i}^{(h-1)}}{N} \text { em que } \quad \boldsymbol{C}_{i}=\left(\boldsymbol{\theta}_{i}^{(h-1)}-\boldsymbol{D}^{(h-1)} \boldsymbol{z}_{i}^{(h-1)}\right) .
\end{aligned}
$$

- Passo 10: Gerar uma amostra $\boldsymbol{\delta}^{(h)}$ de

$$
\boldsymbol{\delta} \mid \ldots \sim p\left(\boldsymbol{\delta} \quad\left\{\quad\left\{\boldsymbol{Z}_{i}^{(h)}, \omega_{i}^{(h-1)}, \boldsymbol{\theta}_{i}^{(h)} ; i=1, \ldots, N\right\}, \boldsymbol{\mu}_{\theta}^{(h)}, \boldsymbol{\Sigma}_{\theta}^{(h)}, \boldsymbol{y}\right)\right.
$$

a partir do seguinte procedimento de Metropolis-Hastings:

1. Simule um valor aleatório $\boldsymbol{\delta}^{*}$ a partir de uma distribuição proposta $q_{\boldsymbol{\delta}}\left(\boldsymbol{\delta} \mid \boldsymbol{\delta}^{(h-1)}\right)$, por exemplo, $q_{\boldsymbol{\delta}}\left(\boldsymbol{\delta} \mid \boldsymbol{\delta}^{(h-1)}\right)=N_{K}\left(\boldsymbol{\delta}^{(h-1)}\right.$, $\left.\operatorname{diag}\left(\boldsymbol{c}_{\boldsymbol{\delta}}^{2}\right)\right)$, com vetor $\boldsymbol{c}_{\boldsymbol{\delta}}^{2}$ adequadamente fixado com o intuito de obter uma taxa de aceitação de aproximadamente $25 \%$ pois segundo Gelman et al. (1996) e Patz e Junker (1999a) isto produz Cadeias de Markov razoavelmente eficientes.

2. Calcule a probabilidade de aceitação

$$
\alpha\left(\boldsymbol{\delta}^{(h-1)}, \boldsymbol{\delta}^{*}\right)=\min \left\{R_{\boldsymbol{\delta}}, 1\right\},
$$

onde:

$$
\begin{aligned}
R_{\boldsymbol{\delta}}= & \frac{\prod_{i=1}^{N}\left\{\exp \left\{-\frac{1}{2}\left(\boldsymbol{\theta}_{i}^{(h)}-\boldsymbol{\mu}_{\theta}^{(h)}-\boldsymbol{D}^{*} \boldsymbol{z}_{i}^{(h)}\right)^{\prime} \omega_{i}^{(h-1)} \boldsymbol{Q}^{(h)}\left(\boldsymbol{\theta}_{i}^{(h)}-\boldsymbol{\mu}_{\theta}^{(h)}-\boldsymbol{D}^{*} \boldsymbol{z}_{i}^{(h)}\right)\right\}\right\}}{\prod_{i=1}^{N}\left\{\exp \left\{-\frac{1}{2}\left(\boldsymbol{\theta}_{i}^{(h)}-\boldsymbol{\mu}_{\theta}^{(h)}-\boldsymbol{D}^{(h-1)} \boldsymbol{z}_{i}^{(h)}\right)^{\prime} \omega_{i}^{(h-1)} \boldsymbol{Q}^{(h)}\left(\boldsymbol{\theta}_{i}^{(h)}-\boldsymbol{\mu}_{\theta}^{(h)}-\boldsymbol{D}^{(h-1)} \boldsymbol{z}_{i}^{(h)}\right)\right\}\right\}} \\
& \times \frac{\exp \left\{-\frac{1}{2}\left(\boldsymbol{\delta}^{*}\right)^{\prime} \boldsymbol{\Gamma}_{0}^{-1} \boldsymbol{\delta}^{*}\right\}}{\exp \left\{-\frac{1}{2}\left(\boldsymbol{\delta}^{(h-1)}\right)^{\prime} \boldsymbol{\Gamma}_{0}^{-1} \boldsymbol{\delta}^{(h-1)}\right\}} .
\end{aligned}
$$

Note que devido a distribuição proposta $q_{\delta}\left(\boldsymbol{\delta} \mid \boldsymbol{\delta}^{(h-1)}\right)$ ser simétrica em seus argumentos a mesma se cancela em $R_{\boldsymbol{\delta}}$, justificando assim a sua "ausência" em (4.13).

3. Simule $u \sim U(0,1)$ e aceite $\boldsymbol{\delta}^{(h)}=\boldsymbol{\delta}^{*}$ com probabilidade $\alpha\left(\boldsymbol{\delta}^{(h-1)}, \boldsymbol{\delta}^{*}\right)$, i.e., aceite se $u \leq \alpha\left(\boldsymbol{\delta}^{(h-1)}, \boldsymbol{\delta}^{*}\right)$, caso contrário, adote $\boldsymbol{\delta}^{(h)}=\boldsymbol{\delta}^{(h-1)}$. 


\subsection{Estimação Bayesiana do Modelo Kd3PP-tA (e Km3PP) via Método} DAGS

- Passo 11: Gerar amostras independentes $\omega_{1}^{(h)}, \ldots, \omega_{N}^{(h)}$ a partir de distribuições independentes

$$
\omega_{i} \mid \ldots \sim \operatorname{Gama}\left(\frac{K+\nu / 2}{2}, \frac{c_{i}}{2}\right),
$$

em que

$$
c_{i}=2\left\{\left(\boldsymbol{\theta}_{i}^{(h)}-\boldsymbol{\mu}_{\theta}^{(h)}-\boldsymbol{D}^{(h)} \boldsymbol{z}_{i}^{(h)}\right)^{\prime} \boldsymbol{Q}^{(h)}\left(\boldsymbol{\theta}_{i}^{(h)}-\boldsymbol{\mu}_{\theta}^{(h)}-\boldsymbol{D}^{(h)} \boldsymbol{z}_{i}^{(h)}\right)+\frac{2}{\nu^{(h-1)}}\right\}^{-1},
$$

$\operatorname{com} \nu^{(h-1)}>2$.

- Passo 12: Gerar uma amostra $\nu^{(h)}$ de

$$
p\left(\nu \mid\left\{\omega_{i}^{(h)} ; i=1, \ldots, N\right\}, \boldsymbol{y}\right)
$$

a partir do seguinte procedimento de Metropolis-Hastings:

1. Simule um valor aleatório $\nu^{*}$ a partir de uma distribuição proposta $q_{\nu}\left(\nu \mid \nu^{(h-1)}\right)$ adequadamente escolhida (Chib e Greenberg (1995), num contexto geral, observaram que esta escolha afeta fortemente a taxa de convergência da Cadeia de Markov para a distribuição estacionária).

2. Calcule a probabilidade de aceitação

$$
\alpha\left(\nu^{(h-1)}, \nu^{*}\right)=\min \left\{R_{\nu}, 1\right\},
$$

onde:

$$
\begin{aligned}
& R_{\nu}=\frac{\prod_{i=1}^{N}\left\{\frac{1}{\Gamma\left(\frac{\nu^{*}}{2}\right)}\left(\frac{2}{\nu^{*}}\right)^{\frac{\nu^{*} / 2}{2}}\left(\omega_{i}^{(h)}\right)^{\frac{\nu^{*}}{2}-1} \exp \left\{-\frac{1}{2} \frac{2}{\nu^{*}} \omega_{i}^{(h)}\right\}\right\}}{\prod_{i=1}^{N}\left\{\frac{1}{\Gamma\left(\frac{\nu^{(h-1)}}{2}\right)}\left(\frac{2}{\nu^{(h-1)}}\right)^{\frac{\nu^{(h-1)} / 2}{2}}\left(\omega_{i}^{(h)}\right)^{\frac{\nu^{(h-1)}}{2}-1} \exp \left\{-\frac{1}{2} \frac{2}{\nu^{(h-1)}} \omega_{i}^{(h)}\right\}\right\}}
\end{aligned}
$$

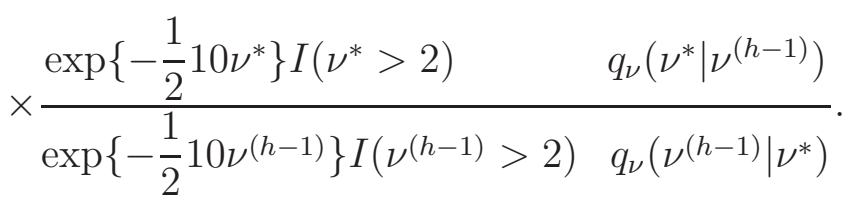

3. Simule $u \sim U(0,1)$ e aceite $\nu^{(h)}=\nu^{*}$ com probabilidade $\alpha\left(\nu^{(h-1)}, \nu^{*}\right)$, i.e., aceite se $u \leq \alpha\left(\nu^{(h-1)}, \nu^{*}\right)$, caso contrário, adote $\nu^{(h)}=\nu^{(h-1)}$. 
- Passo 13: Repetir os passos 2 a 12 até que algum(uns) critério(s) de convergência da cadeia seja $(\mathrm{m})$ satisfeito(s) e um número adequadamente de amostras estejam disponíveis (Gelman et al., 1995; Gamerman; Lopes, 2006).

Com relação ao algoritmo descrito acima, alguns comentários merecem ser considerados. Tais comentários são apresentados logo abaixo.

\subsubsection{Escolha de Valores Iniciais}

Sugestões simples para inicializar os valores dos parâmetros no Passo 1 são:

- $\boldsymbol{u}^{(0)}=\mathbf{0}$ e $v_{i j}^{(0)}=0, \forall i, j$ ou gerados aleatoriamente de distribuição normal padrão;

- $\boldsymbol{\theta}_{i}^{(0)}=\mathbf{0}, \forall i$ ou amostra aleatória de $N_{K}\left(\mathbf{0}, \boldsymbol{I}_{K}\right)$;

- $\boldsymbol{\beta}_{12 j}^{(0)}=(0,1, \ldots, 1)^{\prime}, \quad \beta_{3 j}^{(0)}=0,2 \quad \forall j ;$

- $\boldsymbol{z}_{1}^{(0)}, \ldots, \boldsymbol{z}_{N}^{(0)}$ todos nulos ou amostra independente gerada de distribuição $N_{K}\left(\mathbf{0}, \boldsymbol{I}_{K}\right)$;

- Matriz nula ou identidade para o parâmetro de precisão $\boldsymbol{Q}^{(0)}=\left(\boldsymbol{\Sigma}_{\theta}^{-1}\right)^{(0)}$;

- $\boldsymbol{\mu}_{\theta}^{(0)}=\mathbf{0}$; e

- $\boldsymbol{D}^{(0)}=\operatorname{diag}\left(\boldsymbol{\delta}^{(0)}\right)=\operatorname{diag}(\mathbf{0})=\mathbf{0}_{K}$ sendo uma matriz quadrada nula de dimensão $K$.

Com o intuito de melhorar a convergência de uma única sequência amostral MCMC, algumas extratégias para iniciar o algoritmo também podem ser adotadas. Béguin e Glas (2001), por exemplo, sugerem o uso de estimativas bayesianas tais como modas a posteriori como valores iniciais para os parâmetros dos itens. Tais estimativas podem ser obtidas a partir do uso de softwares comerciais como Bilog-MG (Zimowski et al., 1996) ou de pacotes livremente disponibilizados pelo programa $\mathrm{R}$ ( $\mathrm{R}$ Development Core Team, 2007). Como exemplo, podemos citar o ltm (Rizopoulos, 2006) que permite obter estimativas de máxima verossimilhança marginal de modelos uni e bidimensionais da TRI.

No programa $R$, o método DAGS para a estimação do modelo multidimensional da TRI com distribuição normal para os traços latentes encontra-se implementado na função MCMCirtKd do pacote MCMCpack (Martin; Quinn, 2006) e nesta função a opção padrão ("default") é iniciar os parâmetros dos itens com os coeficientes estimados de vários modelos de regressão logística. Esta opção também é adotada pelo pacote pscl e uma das opções disponíveis para iniciar os parâmetros dos traços latentes consiste em adotar os valores dos autovetores da matriz de correlação dos dados duplamente centralizados. Para

Matos, G. S.

IME/USP 
mais informações sobre esta última opção recomendamos a leitura do manual do pacote pscl disponível em http://www.cran.r-project.org/ ou a leitura de Clinton et al. (2004)).

Na prática, é prudente considerar a execução do algoritmo sob algumas das opções descritas acima, lembrando-se que os valores amostrais obtidos após a convergência da Cadeia de Markov devem corresponder à distribuição de interesse que, em princípio, não deve ser afetada pelo estado inicial da Cadeia.

\subsubsection{Estimação de Sub-Modelos}

Conforme descreveremos abaixo, a versão básica do algoritmo DAGS pode ser facilmente adaptada para o caso em que um ou mais parâmetros são conhecidos, precisam ser fixados em um determinado valor e/ou precisam ficar restritos à alguns valores do espaço paramétrico.

\section{(a) Parâmetro de Acerto Casual $\beta_{3 j}$ Fixado em Valor Não-nulo}

Se por algum motivo o parâmetro de acerto casual $\beta_{3 j}$ de um ou mais item é conhecido ou precisa ser fixado em algum valor não nulo, a modificação necessária na versão básica do algoritmo DAGS consiste em omitir a amostragem do parâmetro $\beta_{3 j}$ (Passo 6 ) associado ao item de interesse (Sahu, 2002).

Vale lembrar que este procedimento poderá ser de grande utilidade na situação em que alguns itens encontram-se previamente calibrados, como aqueles provenientes de Banco de Itens mantidos por Agências/Institutos de Avaliação Educacional (Andrade et al., 2000).

\section{(b) Parâmetro de Acerto Casual $\beta_{3 j}$ Fixado em Valor Nulo}

No caso em que seja razoável adotar FRI's de 2 ou 1 parâmetro para um ou mais dos itens envolvidos em um teste, a adaptação necessária consiste em fixar $u_{i j}=0$ em toda iteração do algoritmo, além de omitir a amostragem do parâmetro $\beta_{3 j}$ no $\quad$ Passo 6 dos itens correspondentes (Sahu, 2002).

\section{(c) Parâmetros de Dificuldade e/ou Discriminação Fixos ou Restritos}

Várias situações podem surgir em que alguns parâmetros associados à dificuldade e/ou discriminação de alguns itens são conhecidos (já calibrados) ou precisam ser fixados ou restritos a algum sub-espaço paramétrico. Na Seção 3.3, por exemplo, vimos a necessidade de se adotar restrições sobre alguns parâmetros de itens com o intuito de contornar possíveis

Matos, G. S.

$\mathrm{IME} / \mathrm{USP}$ 


\subsection{Estimação Bayesiana do Modelo Kd3PP-tA (e Km3PP) via Método} DAGS

problemas causados pela indeterminação da escala e invariância rotacional a que os modelos da TRI estão sujeitos. Outra situação surge em testes da área educacional onde valores não-negativos são esperados para os parâmetros associados à discriminação, pois, nestes casos, espera-se que a probabilidade de resposta correta ao item aumente na medida em que cada um dos componentes do vetor de habilidades (traços latentes) também aumenta (Nojosa, 2002). A necessidade de se fixar parâmetros associados à discriminação também pode surgir se o interesse é estimar os parâmetros do modelo de resposta ao item K-multivariado (Seção 3.2.2) ou os parâmetros do modelo Rasch (Fischer; Molenaar, 1995).

Para que as situações descritas acima possam ser contempladas, algumas modificações no Passo 5 da versão básica do algoritmo DAGS precisam ser desenvolvidas. Para isto, uma das seguintes estratégias pode ser adotada:

i. No contexto da inferência bayesiana uma das maneiras de se restringir (fixar) os valores de alguns parâmetros de um modelo consiste em especificar distribuições a priori bastante precisas no sentido de especificar distribuições com valores esperados iguais aos valores que se deseja fixar com uma variabilidade mínima em torno destes valores. No contexto do modelo multidimensional de 2 parâmetros probito da TRI, este procedimento é adotado, por exemplo, por Jackman (2001) e Clinton et al. (2004).

ii. Um segundo procedimento consiste em não especificar distribuições a priori para os parâmetros fixados do $j$-ésimo item que denotaremos por um sub-vetor $\boldsymbol{\beta}_{j}$ e conseqüentemente não amostrar destes no Passo $\mathbf{5}$ mas simplesmente amostrar da distribuição normal associada ao sub-vetor dos parâmetros não fixados, $\boldsymbol{\beta}_{j o}$, do $\boldsymbol{j}$ ésimo item. Tudo isto sem levar em consideração a informação dos valores assumidos pelos parâmetros fixados $\boldsymbol{\beta}_{j \bullet}$.

iii. Uma terceira alternativa descrita em Quinn (2004) e implementada no pacote MCMCpack do R (Martin; Quinn, 2006), consiste em considerar na distribuição condicional completa do Passo $\mathbf{5}$ a informação dos valores assumidos pelos parâmetros fixados do $j$-ésimo item, $\boldsymbol{\beta}_{j}$. Deste modo, a seguinte distribuição condicional é obtida:

$$
\boldsymbol{\beta}_{j \circ} \mid \ldots, \boldsymbol{y} \sim N_{K_{\circ}}\left(\boldsymbol{\varphi}_{j \circ} \boldsymbol{m}_{j \circ}, \boldsymbol{\varphi}_{j \circ}\right)
$$

em que $\boldsymbol{\varphi}_{j \circ}=\left(\boldsymbol{B}_{0, j \circ}^{-1}+\boldsymbol{\Theta}^{(j \circ)^{\prime}} \boldsymbol{\Theta}^{(j \circ)}\right)^{-1}$ e $\quad \boldsymbol{m}_{j \circ}=\left(\boldsymbol{B}_{0, j \circ}^{-1} \boldsymbol{b}_{0, j \circ}+\boldsymbol{\Theta}^{(j \circ)^{\prime}} \boldsymbol{v}_{j \bullet}\right)$ com

- $\boldsymbol{\beta}_{j \circ}$ representando o vetor de parâmetros livres do $j$-ésimo item, ou seja, da j-ésima linha da matriz de parâmetros dos itens $\boldsymbol{\beta}=\left(\boldsymbol{\beta}_{1}, \boldsymbol{\beta}_{2}\right)$.

Matos, G. S.

$\mathrm{IME} / \mathrm{USP}$ 


\subsection{Estimação Bayesiana do Modelo Kd3PP-tA (e Km3PP) via Método} DAGS

- $\Theta^{(j \circ)}$ é uma matriz $n_{j} \times K_{\circ}$ formada apenas pelos elementos da matriz $\Theta^{(j)}$ que são multiplicados pelos elementos $\boldsymbol{\beta}_{j \circ}$ na expressão $\boldsymbol{\Theta}^{(j)} \boldsymbol{\beta}_{12 j}$. Note que $\boldsymbol{\Theta}^{(j)}$ é tal como descrita no Passo 5 do algoritmo DAGS e Seção A.7 do Apêndice A. Assim, $K_{\circ} \leq(1+K)$ representa a quantidade de parâmetros livres do $j$-ésimo item.

- $\boldsymbol{B}_{0, j_{\circ}}$ e $\boldsymbol{b}_{0, j_{\circ}}$ são os hiperparâmetros da distribuição a priori especificada somente para os parâmetros livres do $j$-ésimo item; e

$-\boldsymbol{v}_{j \bullet}=\boldsymbol{v}_{j}-\boldsymbol{\Theta}^{(j \bullet)} \boldsymbol{\beta}_{j \bullet}$ é um vetor de dimensão $n_{j}$ em que $\boldsymbol{\Theta}^{(j \bullet)}$ é uma matriz $n_{j} \times$ $K$ • formada apenas pelos elementos da matriz $\boldsymbol{\Theta}^{(j)}$ que $\boldsymbol{n} \tilde{\boldsymbol{a}} \boldsymbol{o}$ são multiplicados pelos elementos $\boldsymbol{\beta}_{j \circ}$ na expressão $\boldsymbol{\Theta}^{(j)} \boldsymbol{\beta}_{12 j}$. O vetor $\boldsymbol{\beta}_{j \bullet}$ é formado, portanto, somente pelos parâmetros fixos do $j$-ésimo item cuja dimensão é dada por $K_{\bullet}=(1+K)-K_{\circ}$.

Finalmente, quando restrições nos parâmetros na forma de desigualdade são necessárias, uma opção é amostrar de $\boldsymbol{\beta}_{j \circ}$ repetidamente até que a restrição desejada seja satisfeita.

\section{(d) Estimação de "Blocos" de Parâmetros}

Para os casos em que não existe interesse em realizar a estimação simultânea dos parâmetros dos itens $\boldsymbol{\beta}$, traços latentes $\boldsymbol{\theta}_{1}, \ldots, \boldsymbol{\theta}_{N}$ e populacionais $\boldsymbol{\eta}=\left(\boldsymbol{\mu}_{\theta}, \boldsymbol{\Sigma}_{\theta}, \boldsymbol{D}, \boldsymbol{\omega}, \nu\right)$ mas apenas sobre um ou dois destes "blocos" de parâmetros, o procedimento natural é eliminar da distribuição a posteriori (4.6) a distribuição a priori do(s) bloco(s) de parâmetros conhecidos e não realizar a amostragem correspondente no algotimo Gibbs. Tal procedimento é semelhante à estimação por máxima verossimilhança em "blocos" descrita em Tavares (2001).

\section{(e) Modelos Unidimensionais}

Uma interessante modificação na versão básica do algoritmo DAGS surge quando o modelo a ser estimado recai sobre o caso especial dos modelos unidimensionais, ou seja, quando os traços latentes podem ser considerados de dimensão $K=1$. Neste caso, a amostragem por Metropolis-Hastings do parâmetro associado à assimetria (Passo 10) pode ser substituída pela amostragem direta da distribuição

$$
\delta \mid \ldots \sim N_{1}\left(\Psi_{\delta} \psi_{\delta}, \Psi_{\delta}\right)
$$

em que

Matos, G. S.

$\mathrm{IME} / \mathrm{USP}$ 
- $\Psi_{\delta}=\left(\gamma_{0}^{-1}+\boldsymbol{z}^{\prime} \Sigma_{\delta}^{-1} \boldsymbol{z}\right)^{-1}$

$$
\operatorname{com} \boldsymbol{z}=\left(z_{1}, \ldots, z_{N}\right)^{\prime} \quad \text { e } \quad \Sigma_{\delta}^{-1}=\frac{1}{\sigma_{\theta}^{2}} \operatorname{diag}\left(\omega_{1}, \ldots, \omega_{N}\right)
$$

- $\psi_{\delta}=\gamma_{0}^{-1} .0+\boldsymbol{z}^{\prime} \Sigma_{\delta}^{-1} \boldsymbol{\theta}^{*}=\boldsymbol{z}^{\prime} \Sigma_{\delta}^{-1} \boldsymbol{\theta}^{*}$, $\operatorname{com} \boldsymbol{\theta}^{*}=\left(\theta_{1}-\mu_{\theta}, \ldots, \theta_{N}-\mu_{\theta}\right)^{\prime}=\boldsymbol{\theta}-\mu_{\theta} \mathbf{1}_{N}$.

As expressões necessárias para se chegar a esta distribuição enconcontram-se na Seção A.10 do Apêndice A.

\subsubsection{Metropolis-Hastings e Distribuições Discretas}

É interessante notar que rigorasamente falando, a versão básica do algoritmo DAGS deveria ser chamada de algoritmo de Metropolis-Hastings dentro de Gibbs, pois, como podemos perceber, nos Passos 10 e $\mathbf{1 2}$ precisamos amostrar os parâmetros por MetropolisHastings (Patz; Junker, 1999a).

Apesar de Tierney (1994) ter desenvolvido estudos que validam a convergência de algoritmos do tipo Metropolis-Hastings dentro de Gibbs, não devemos negar que é sempre desejável a obtenção de distribuições fechadas para as distribuições condicionais completas. Com este intuito a amostragem do grau de liberdade $\nu$ por Metropolis-Hastings no Passo 12 pode ser substituída pela simples geração de números aleatórios de distribuições discretas, conforme descrito por Albert e Chib (1993). Tal procedimento também pode ser considerado para o caso do vetor de parâmetros de assimetria $\boldsymbol{\delta}$, sendo necessário, é claro, a especificação adequada de uma distribuição multivariada discreta a priori.

\subsubsection{Extensão do Algoritmo para Modelos de Grupos Múltiplos}

Em situações reais de avaliação pode ser de interesse a comparação entre diferentes grupos de respondentes através da aplicação de testes compostos por items completamente ou parcialmente comuns (Andrade et al., 2000, Cap. 5). A adaptação da versão básica do algoritmo DAGS para a realização de tais comparações (ou equalizações) pode ser realizada de maneira análoga à proposta por Béguin e Glas (2001).

\subsubsection{Implementação Computacional}

Para a implementação computacional da metodologia descrita neste capítulo aplicada a alguns modelos discutidos aqui, optamos por realizar adaptações nos códigos abertos do pacote MCMCpack (Martin; Quinn, 2006) do ambiente computacional e estatístico R.

Matos, G. S.

IME/USP 
Algumas vantagens deste procedimento seguem do fato da computação intensiva ser executada na linguagem de programação $\mathrm{C}++$ através da Biblioteca Estatística $\boldsymbol{S} \boldsymbol{c y t h} \boldsymbol{e}$ que permite gerar números aleatórios, realizar operações matriciais e armazenar dados de maneira relativamente simples e eficiente (Martin e Quinn, 2000, http://scythe.wustl.edu/). Vale dizer que esta biblioteca também é livremente disponibilizada e seu uso também pode servir à implementação de procedimentos inferenciais no contexto freqüentista. Outra vantagem da abordagem escolhida para a implementação de nosso algoritmo segue do fato das amostras da distribuição a posteriori ficarem disponíveis no formato de objetos $\boldsymbol{m c m} \boldsymbol{c}$ permitindo assim a realização de análises de convergência através do pacote $\operatorname{coda}$ do $\boldsymbol{R}$. Outro pacote do R que potencializa uma análise bayesiana é o rv ("Random Variable") que permite a obtenção simples e rápida de várias medidas de interesse, além de gráficos de credibilidade. Tudo isto num único ambiente gráfico e computacional, o $\mathrm{R}$ (R Development Core Team, 2007).

Os procedimentos necessários para realizar as devidas modificações e compilação do pacote MCMCpack (Martin; Quinn, 2006) podem ser encontrados no manual de desenvolvedores do MCMCpack, disponível em:

http://mcmcpack.wustl.edu/wiki/index.php/MCMCpack_Development_Page.

Outra informação importante diz respeito ao ambiente do Sistema Linux (e.g. Distribuidor Ubuntu) pois foi somente com o uso de um Sistema Linux que particularmente encontramos menores dificuldades para realizar todos os procedimentos de modificação e compilação do pacote $R$. Além disso, é importante dizer que todas as modificações/adaptações dos códigos foram feitas na versão MCMCpack_0.8-2 do pacote MCMCpack. No Apêndice B apresentamos informações sobre como um usuário R pode ter acesso à função $M C M C i r t K d 2 P P S n$ que desenvolvemos com o intuito de realizar análises no próximo Capítulo.

No próximo capítulo, alguns estudos de dados simulados são desenvolvidos com o intuito de investigar a aplicabilidade de alguns modelos multidimensionais da TRI até aqui discutidos e estendidos. Ter uma noção sobre a eficiência do método de estimação bayesiana via método DAGS discutido neste capítulo também é um dos objetivos das análises de dados simulados a serem descritos.

Matos, G. S.

$\mathrm{IME} / \mathrm{USP}$ 
Capítulo 5

\section{Análises Envolvendo Dados Simulados}

\subsection{Introdução}

Neste capítulo aplicamos a alguns conjuntos de dados (testes) simulados a metodologia de estimação bayesiana desenvolvida no capítulo anterior. O principal objetivo destas aplicações é investigar a eficiência da metodologia além de possíveis vantagens e/ou desvantagens em se analisar testes com a informação prévia de que o comportamento dos traços latentes de uma determinada população possa ser representado por uma distribuição assimétrica. Neste contexto, é importante lembrar que a especificação de uma distribuição assimétrica para representar o comportamento dos traços latentes, à rigor, não define um Modelo de Resposta ao Item (MRI; Seção 2.1) pois, de fato, a especificação desta ou de outra distribuição surge como um elemento necessário ao desenvolvimento e implementação de um processo de estimação dos parâmetros de um MRI, seja através de um método bayesiano como desenvolvido no Capítulo 4 ou de um método freqüentista com o uso da função de verossimilhança marginal discutido na Seção 3.3.1.2.

Nas próximas seções apresentaremos dois tipos de teste que diferem quanto à dimensionalidade, ou seja, quanto à quantidade e/ou intensidade de traços latentes exigidos dos respondentes para se obter uma determinada resposta ao item (e conjunto de itens). Na Seção 5.2, com o intuito de nos familiarizarmos com a execução do processo de estimação e interpretação dos modelos de resposta ao item com o uso de distribuições assimétricas para os traços latentes, idealizamos a aplicação de um teste constituído somente por itens do tipo unidimensional os quais serão analisados sob o modelo 1d2PPN e 1d2PPSn, ou seja, o teste será analisado sob o modelo 1-dimensional de 2 Parâmetros Probito asssumindo duas distribuições a priori para os traços latentes: Normal e "Skew-normal". Tais modelos serão aplicados quando a verdadeira distribuição dos traços latentes é normal assimétrica com parâmetros de localização $\mu=0$, escala $\sigma=1$ e assimetria $\delta=-1.5$ denotada por $\operatorname{Sn}(0,1,-1.5)$. Na Seção 5.3 idealizamos um teste constituído por 30 itens do tipo bididimensional e neste caso os dados serão analisados usando os modelos $2 \mathrm{~d} 2 \mathrm{PPN}$, 2d2PPSn, 2m2PPN e 2m2PPSn. De maneira análoga à primeira análise de dados simulados, os modelos aplicados nesta seção serão avaliados e comparados na situação em que a verdadeira distribuição dos traços latentes é normal bivariada assimétrica. Na Sub-seção 
5.3.1 apresentamos uma descrição detalhada dos parâmetros desta distribuição e na Seção 5.4 uma descrição geral sobre os resultados observados no capítulo é apresentada.

\subsection{Dados Simulados 1: Teste Unidimensional}

O primeiro conjunto de dados simulado é constituído por $J=30$ itens (unidimensionais) aplicados a uma quantidade moderada de respondentes, ou seja, $N=400$. Para caracterizar os itens foram considerados vários níveis de dificuldade entre fáceis e difíceis, isto é, entre os valores -2.2 e 2.2 que podem ser observados na Tabela 5.1. Com relação a estes valores é importante lembrar que os mesmos correspondem a uma métrica associada a uma distribuição dos traços latentes simétrica, e.g. normal, com parâmetros de localização $\mu=0$ e escala $\sigma=1$. Lembramos, ainda, que diferentemente da distribuição normal, o valor esperado e o desvio-padrão da normal assimétrica (Sahu et al., 2003) não coincidem, respectivamente, com estes parâmetros. Isto porque tais características dependem do parâmetro de assimetria, $\delta$, da seguinte forma: $E(X)=\mu+\left(\frac{2}{\pi}\right)^{\frac{1}{2}} \delta$ e $\operatorname{Var}(X)=\sigma^{2}+\left(1-\frac{2}{\pi}\right) \delta^{2}$.

No que conscerne a caracterização dos itens quanto ao poder de discriminação, valores entre 0,55 e 1,5 (Tabela 5.1) foram adotados pois estes são esperados quando os modelos de resposta ao item (função de ligação) baseam-se na função de distribuição normal (ogiva normal) (Nojosa, 2001; Baker; Kim, 2004).

Ainda com relação aos valores considerados para os parâmetros dos itens (unidimensionais), Baker e Kim (2004) lembram que a parametrização em termos de inclinação $\beta_{2 j}$ e intercepto $\beta_{1 j}$ dada por $\eta_{i j}=\beta_{2 j} \theta_{i}-\beta_{1 j}$ é muito mais utilizada na literatura técnica e computacional da TRI. Por outro lado, é a parametrização de Lord e Novick (1968) dada por $\eta_{i j}=a_{j}\left(\theta_{i}-b_{j}\right)$ que deve ser preferida para descrever as características dos itens em termos práticos pois é somente nesta parametrização que os parâmetros $b_{j}$ e $a_{j}$ corretamente representam a localização e a inclinação de uma Curva Característica do Item (CCI) ou, equivalentemente, a dificuldade e a discriminação de um item (McDonald (1997b) também discute este assunto). Vale destacar, ainda, que é somente nesta última parametrização que o parâmetro de dificuldade $b_{j}$ fica representado na mesma escala do traço latente $\theta$.

Diante das considerações descritas acima, apresentamos na Tabela 5.1 os verdadeiros parâmetros dos itens associados ao modelo 1d2PP (unidimensional de 2 parâmetros probito; Seção 3.2.1) conforme a parametrização de Lord e Novick (1968), ressaltando, porém, que para efeito de simulação das respostas aos itens os mesmos foram transformados para a parametrização em termos de inclinação $\beta_{2 j}$ e intercepto $\beta_{1 j}$, ou seja, $\beta_{2 j}=a_{j}$ e $\beta_{1 j}=a_{j} b_{j}$. Por outro lado, para efeito de comparação entre as estimativas obtidas sob esta última parametrização e os verdadeiros valores, a transformação inversa foi aplicada às estimativas de modo que todas as interpretações relativas à discriminação $a_{j} \mathrm{e}$

Matos, G. S.

$\mathrm{IME} / \mathrm{USP}$ 
Tabela 5.1 Verdadeiros parâmetros dos itens (unidimensionais e de 2 parâmetros) considerando a ogiva normal como modelo de resposta ao item e a parametrização de Lord e Novick (1968).

\begin{tabular}{lrrrrrrrrrr}
\hline \hline & \multicolumn{1}{c}{ Item } \\
\cline { 2 - 10 } Parâmetro & $\mathrm{i} 1 \mathrm{v}$ & $\mathrm{i} 2 \mathrm{v}$ & $\mathrm{i} 3 \mathrm{v}$ & $\mathrm{i} 4 \mathrm{v}$ & $\mathrm{i} 5 \mathrm{v}$ & $\mathrm{i} 6 \mathrm{v}$ & $\mathrm{i} 7 \mathrm{v}$ & $\mathrm{i} 8 \mathrm{v}$ & $\mathrm{i} 9 \mathrm{v}$ & $\mathrm{i} 10 \mathrm{v}$ \\
\hline $\mathrm{b}$ & -2.20 & -2.02 & -1.85 & -1.68 & -1.51 & -1.34 & -1.17 & -1.00 & -0.80 & -0.67 \\
$\mathrm{a}$ & 0.55 & 1.16 & 0.89 & 1.26 & 1.42 & 0.63 & 1.28 & 0.69 & 1.44 & 0.90 \\
\hline & $\mathrm{i} 11 \mathrm{v}$ & $\mathrm{i} 12 \mathrm{v}$ & $\mathrm{i} 13 \mathrm{v}$ & $\mathrm{i} 14 \mathrm{v}$ & $\mathrm{i} 15 \mathrm{v}$ & $\mathrm{i} 16 \mathrm{v}$ & $\mathrm{i} 17 \mathrm{v}$ & $\mathrm{i} 18 \mathrm{v}$ & $\mathrm{i} 19 \mathrm{v}$ & $\mathrm{i} 20 \mathrm{v}$ \\
\hline \hline $\mathrm{b}$ & -0.55 & -0.43 & -0.30 & -0.18 & -0.06 & 0.06 & 0.18 & 0.30 & 0.43 & 0.55 \\
$\mathrm{a}$ & 1.00 & 1.38 & 1.13 & 0.78 & 0.82 & 1.23 & 1.33 & 1.07 & 0.74 & 1.28 \\
\hline \hline & $\mathrm{i} 21 \mathrm{v}$ & $\mathrm{i} 22 \mathrm{v}$ & $\mathrm{i} 23 \mathrm{v}$ & $\mathrm{i} 24 \mathrm{v}$ & $\mathrm{i} 25 \mathrm{v}$ & $\mathrm{i} 26 \mathrm{v}$ & $\mathrm{i} 27 \mathrm{v}$ & $\mathrm{i} 28 \mathrm{v}$ & $\mathrm{i} 29 \mathrm{v}$ & $\mathrm{i} 30 \mathrm{v}$ \\
\hline $\mathrm{b}$ & 0.67 & 0.80 & 1.00 & 1.17 & 1.34 & 1.51 & 1.68 & 1.85 & 2.02 & 2.20 \\
$\mathrm{a}$ & 1.05 & 0.71 & 0.84 & 1.21 & 0.98 & 1.42 & 1.32 & 1.05 & 1.18 & 0.80 \\
\hline \hline
\end{tabular}

dificuldade $b_{j}$ ficassem claramente asseguradas (Lord; Novick, 1968; McDonald, 1997b; Baker; Kim, 2004). Além disso, foi de extrema importância a transformação (ou equalização) das estimativas para a métrica dos verdadeiros parâmetros pois, sem isto, viéses foram erroneamente observados uma vez que distribuições na forma padrão (simétrica e assimétrica) foram especificadas para os traços latentes com o objetivo de resolver o problema da falta de identificabilidade (ver Seção 5.2.2), induzindo, assim, escalas de medida (ou métricas) não comparáveis entre os modelos e verdadeira métrica definida pelos parâmetros dos itens originais. Alguns textos que dedicam comentários sobre a relação entre identificabilidade e a necessidade de se transformar a métrica das estimativas para a métrica dos parâmetros originais são: De Ayala e Sava-Bolesta (1999) e Harwell et al. (1996); além da Seção 2.2.1 do presente trabalho. O método de equalização a posteriori (pós-obtenção das estimativas) adotado na análise dos dados desta seção foi o Média-Desvio (Mean-Sigma) que encontra-se descrito, por exemplo, em Andrade et al. (2000). Destacamos, ainda, que alguns autores eliminam a necessidade de uma equalização a posteriori fazendo com que as distribuições originais dos traços latentes (simétricas ou assimétricas) tenham média e desvio-padrão igual a 0 e 1, respectivamente. Evitar uma possível confusão entre o erro de equalização e o erro de estimação é a grande vantagem deste procedimento mas aparentemente só é válido quando o método de estimação em estudo produz estimativas para os traços latentes com média e desvio-padrão também fixados em 0 e 1, respectivamente (DeMars, 2003). Como é possível observar, este não é necessariamente o caso do método 
desenvolvido no presente trabalho, pois no caso da adoção de uma distribuição assimétrica (a priori) para os traços latentes, os parâmetros de localização e escala especificados em 0 e 1, respectivamente, não equivalem à média e desvio-padrão. Além disso, como poderá ser visto, a equalização a posteriori aqui adotada apresenta resultados aceitáveis, até mesmo porque relações lineares entre as estimativas e os verdadeiros parâmetros foram observadas através de investigações informais, o que é desejável para uma maior confiabilidade sobre a equalização realizada em nosso trabalho (Andrade et al., 2000).

\subsubsection{Distribuição dos traços latentes}

A análise de dados simulados que descreveremos em seguida tem por objetivo investigar possíveis vantagens e/ou desvantagens devido à adoção de uma distribuição a priori assimétrica para representar o comportamento populacional dos traços latentes. No entanto, até o momento não mencionamos nada sobre como reconhecer uma distribuição assimétrica com um nível de assimetria significativamente não nulo e com intensidade suficiente para interferir (ou não) nas estimativas obtidas a partir de um determinado método de estimação e inferência. Com o intuito de introduzir esta noção, ilustramos na Figura 5.1, a forma de algumas densidades normais assimétricas padrão $(\mu=0$ e $\sigma=1)$ segundo a proposta de Sahu et al. (2003). Valores esperados e desvios-padrão teóricos também são apresentados. A partir desta figura é possível observar desde uma distribuição com assimetria nula (gráfico (a)), isto é, com o parâmetro $\delta=0$ e, portanto, coincidente com a distribuição normal, até uma distribuição com nível de assimetria relativamente alto e negativo $(\delta=-2.5$, gráfico $(\mathrm{d}))$. Na Figura 5.1 também apresentamos para cada distribuição um histograma e algumas medidas descritivas relacionadas a uma amostra aleatória de tamanho 400 .

A partir da observação da Figura 5.1 e com base em testes clássicos sobre a nulidade da assimetria de cada uma das amostras ilustradas é possível concluir que valores do parâmetro de forma (associado à assimetria), $\delta$, próximos de -1.5 representam intensidade moderada de assimetria. Sendo assim, decidimos considerar no presente estudo a geração dos traços latentes a partir de uma distribuição $\operatorname{Sn}(0,1,-1.5)$ (amostra ilustrada no Gráfico (c) da Figura 5.1).

Como já mencionado, as respostas aos itens gerados sob as condições descritas até aqui serão analisadas sob os modelos 1d2PPN e 1d2PPSn. Esperamos assim, poder detectar algumas vantagens e/ou desvantagens ao se adotar tais modelos no processo de estimação e inferência bayesiana via método DAGS, quando a verdadeira distribuição dos traços latentes possui um nível moderado de assimetria representada pela distribuição $\operatorname{Sn}(0,1,-1.5)$.

Matos, G. S.

IME/USP 


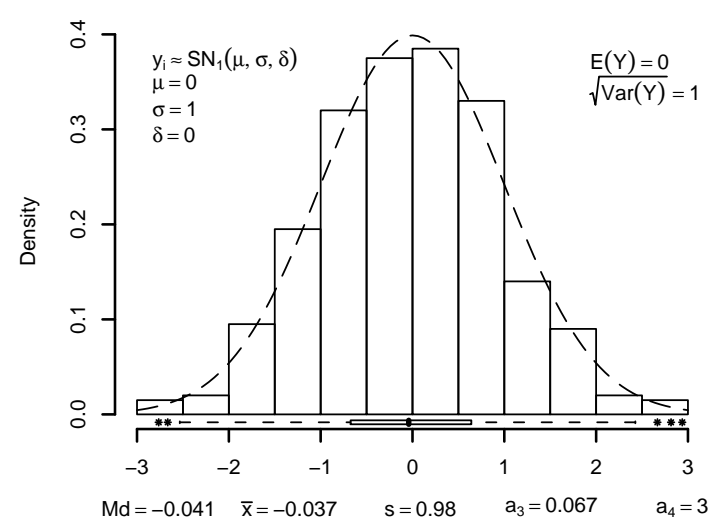

(a)

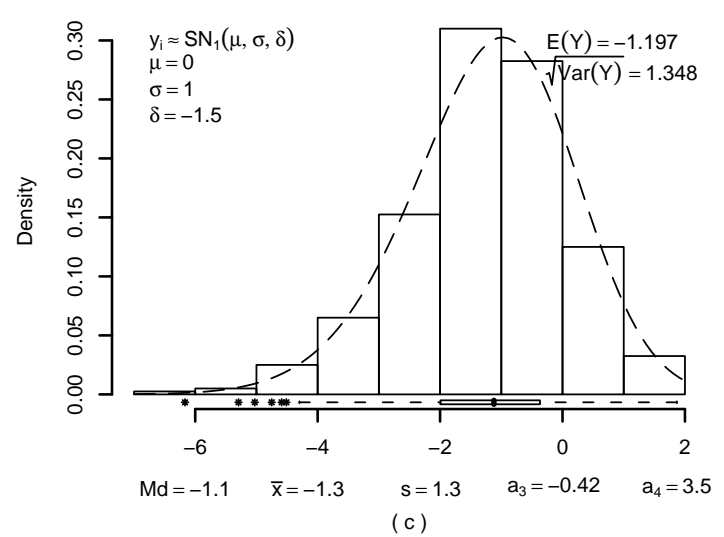

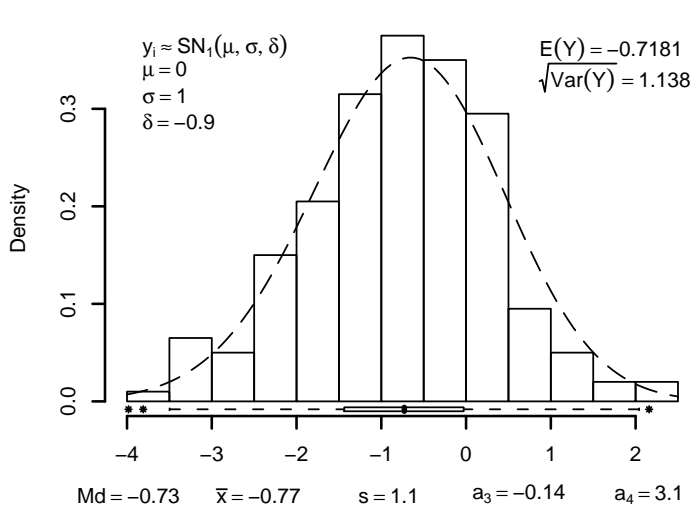

(b)

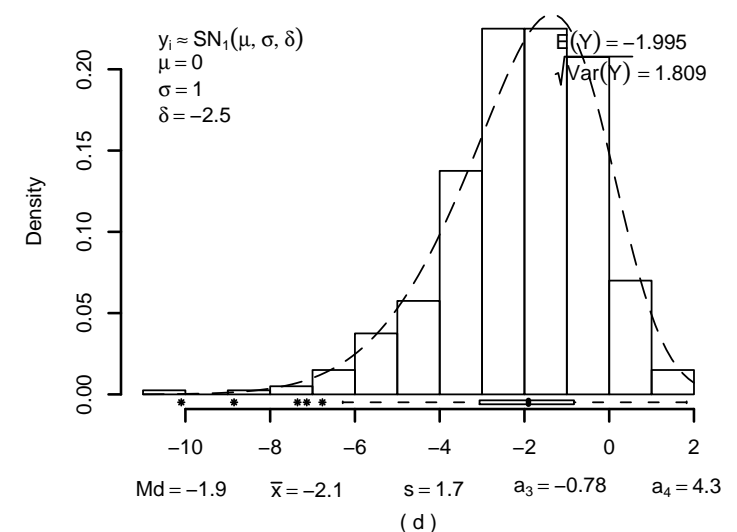

(d)

Figura 5.1 Histogramas, resumos amostrais (mediana, média, desvio-padrão, a3: assimetria e a4: curtose), curvas e valores teóricos segundo algumas distribuições normais assimétricas univariadas Sahu et al. (2003). (As amostras ilustradas nos gráficos (c) e (d) evidenciaram significativamente a rejeição das hipóteses de normalidade, assimetria e curtose. Tais distribuições exemplificam níveis de assimetria moderada e alta, respectivamente.)

\subsubsection{Especificação das Prioris e Identificabilidade dos Modelos}

No presente estudo, o vetor de médias e a matriz de precisão da distribuição a priori (4.5) dos parâmetros de dificuldade e discriminação foram fixados em $\boldsymbol{b}_{0, j}=\left(\mu_{\beta_{1} j}, \mu_{\beta_{2} j}\right)^{\prime}=$ $(0,1)^{\prime}$ e $\boldsymbol{B}_{0, j}^{-1}=\operatorname{diag}\left(\sigma_{\beta_{1} j}^{2}, \sigma_{\beta_{2} j}^{2}\right)^{-1}=\operatorname{diag}(2,0.5)^{-1}=\operatorname{diag}(0.5,2)$, para $j=1, \ldots, 30$. A especificação destes valores, além de considerar a escala $(0,1)$ da distribuição dos traços letentes descrita no próximo parágrafo, também considerou como referência as experiências bem sucedidas de Patz e Junker (1999a), Sahu (2002) e Bazán (2005). Sahu (2002), por exemplo, observou que valores maiores para as variâncias (precisões menores) resul-

Matos, G. S.

$\mathrm{IME} / \mathrm{USP}$ 
tam em estimativas instáveis e Bazán (2005), analisando um teste de matemática aplicado à alunos de escolas Peruanas, encontrou resultados insensíveis quando diferentes valores para estes hiper-parâmetros foram atribuídos, ou seja, insensíveis no sentido de que diferentes parâmetros das distribuições a priori resultaram em inferências bastante similares. Para finalizar a especificação da distribuição a priori do parâmetro de discriminação nós consideramos um truncamento à esquerda do zero, garantindo, portanto, a positividade dos mesmos.

Com relação à distribuição conjunta a priori dos traços latentes, distribuições normal padrão independentes foram consideradas quando adotado o modelo $1 \mathrm{~d} 2 \mathrm{PPN}$ e distribuições normal padrão assimétricas independentes quando adotado o modelo 1d2PPSn. Lembramos que a adoção de distribuições padrão para os traços latentes tem como principal objetivo a identificabilidade local (estabelecimento de uma métrica) do modelo unidimensional de 2 parâmetros probito (2PP) que aliado à restrição de positividade dos parâmetros de discriminação garante a identificabilidade global (ver discussão na pág. 25). Finalmente, para o caso do modelo 1d2PPSn uma distribuição normal padrão foi considerada para a priori do parâmetro de assimetria, $\delta$.

\subsubsection{Análise de Convergência das Cadeias de Markov}

Após realizar análises preliminares sobre a convergência das cadeias de Markov associadas à distribuição a posteriori do modelo $1 \mathrm{~d} 2 \mathrm{PPN}$ e $1 \mathrm{~d} 2 \mathrm{PPSn}$, decidimos por considerar uma amostra de tamanho 1.000 obtida a partir do descarte de 100.000 valores iniciais de uma sequência de 60.0000 valores. Saltos de tamanho 500, portanto, foram realizados. O tempo de processamento para a geração de cada uma destas cadeias foi de aproximadamente $2 \mathrm{hs}$ e 30 min. em um computador Core 2 Dual com 2 Gb de memória RAM e para inicializar a cadeia foram considerados: (i) estimativas de máxima verossimilhança marginal dos parâmetros de dificuldade e de discriminação obtidas a partir do pacote 1 tm do programa $R$ e (ii) traços latentes obtidos a partir de autovetores de uma matriz de correlação de dados duplamente centralizados, conforme descrito no manual do pacote pscl do ambiente computacional e estatístico R (ver também Clinton et al. (2004)). No caso da cadeia associada ao modelo 1d2PPSn, estes mesmos critérios de inicialização foram considerados além do valor nulo para inicializar o parâmetro de assimetria.

A estratégia para se obter uma amostra da distribuição a posteriori a partir de uma longa sequência da cadeia e grandes saltos foi motivada pela observação preliminar de sequências de valores altamente dependentes (autocorrelação alta) com correspondentes tamanhos efetivo de amostra* pequenos. Gamerman e Lopes (2006, p. 149), por exemplo, relatam que em casos como estes a cadeia simulada deve ser suficientemente longa

* O tamanho efetivo de amostra que nos referimos é mais conhecido por "Effective Sample Size" cuja sigla é ESS.

Matos, G. S.

$\mathrm{IME} / \mathrm{USP}$ 
para que o espaço paramétrico de interesse seja adequadamente representado pela amostra MCMC obtida. Diante desta discussão, é interessante lembrar também que a presença de altas autocorrelações observadas em nossa análise preliminar de convergência já era esperada, tendo em vista o uso de variáveis latentes auxiliares no modelo, isto é, de dados aumentados (Sahu, 2002; Chen et al., 2000; Bazán, 2005). É interessante lembrar, ainda, que o uso de saltos numa cadeia de sequência única tem por principais vantagens: (i) a facilidade de se calcular variâncias de estimadores obtidos a partir da amostra da distribuição a posteriori sob a hipótese de uma amostra aproximadamente independente e (ii) o ganho computacional em termos de memória necessária para se armazenar e manipular grandes matrizes de dados (Carlin; Louis, 1996; Gamerman; Lopes, 2006). Por outro lado, Gamerman e Lopes (2006), com base em resultados da Teoria Ergódica, lembram que tal procedimento é dispensável para se obter a convergência de estimativas médias para os verdadeiros valores de interesse. Carlin e Louis (1996, pag. 195, 196) também lembram que o procedimento de saltos numa cadeia sempre aumenta a variância dos estimadores obtidos por média amostral. Uma estratégia alternativa para a obtenção dos valores amostrais da distribuição a posteriori seria através da geração de múltiplas cadeias de Markov a qual não foi considerada em nosso trabalho. Sinharay (2004) é um dos poucos autores que relatam muito bem as experiências adquiridas com relação a convergência de cadeias MCMC na área psicométrica e para concluir esta discussão é importante dizer que todos os autores aqui citados reconhecem a não existência de um procedimento amplamente aceitável pelos pesquisadores.

Uma vez obtida a amostra da distribuição a posteriori sob as condições descritas no parágrafo inicial desta seção, gráficos de trajetória e estatísticas de Geweke (1992) obtidos(as) com o uso do pacote coda (Plummer et al., 2007) forneceram indícios de convergência das cadeias associadas aos modelos (parâmetros) 1d2PPN e 1d2PPSn. Ressaltamos apenas que a cadeia associada ao parâmetro de assimetria foi a que apresentou maior dificuldade para percorrer o espaço paramétrico de interesse devido à alta autocorrelação (ver Figura 5.2).
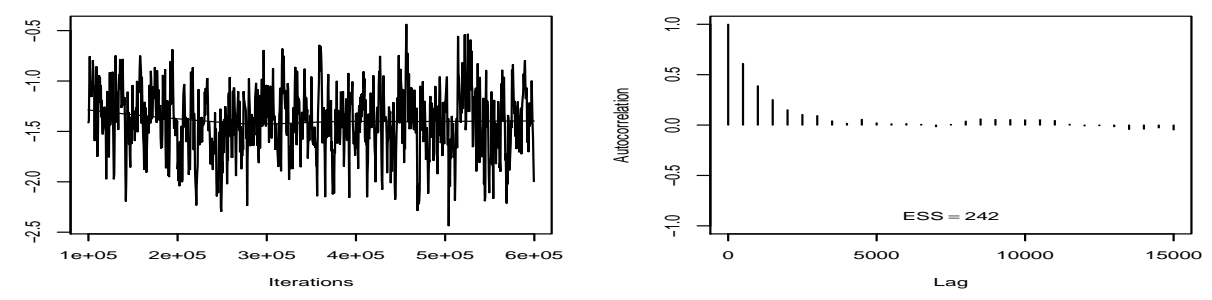

Figura 5.2 Gráficos de trajetória e de autocorrelação do parâmetro de assimetria $\delta$ do modelo $1 d 2 P P S n$.

Matos, G. S.

IME/USP 


\subsubsection{Análise das Estimativas Obtidas sob os Modelos 1d2PPN e 1d2PPSn}

Na Figura 5.3 encontram-se diversos gráficos ilustrando o comportamento das estimativas obtidas sob o modelo 1d2PPN quando o verdadeiro modelo gerador das respostas aos itens é o 1d2PPSn. Como as estimativas obtidas pelo modelo 1d2PPSn apresentaram comportamento bastante semelhante, nos deteremos apenas à análise dos resultados expressos nesta figura, além de algumas medidas comparativas entre as estimativas dos dois modelos. Nos gráficos (a), (b) e (c) apresentamos intervalos quantílicos de $95 \%$ de credibilidade das diferenças (desvios ou resíduos) entre os valores da amostra da distribuição a posteriori e os verdadeiros valores dos parâmetros de dificuldade, discriminação e traços latentes, respectivamente. Os pequenos pontos que se encontram no centro de cada intervalo de credibilidade correspondem à média dos desvios. No gráfico (d) é possível observar a relação entre as médias da amostra da distribuição a posteriori e os verdadeiros valores dos traços latentes dos respondentes.

De modo geral é possível observar que todos os parâmetros são estimados de modo satisfatório tendo em vista que a maioria dos intervalos de credibilidade contém o valor nulo que corresponde a um viés nulo. De forma mais específica, aproximadamente 96,6\% dos intervalos correspondentes aos parâmetros de dificuldade contiveram o valor nulo quando considerado o modelo 1d2PPN. Por outro lado, este percentual foi de $100 \%$ quando considerado o modelo 1d2PPSn. Resultado inverso ocorreu com relação aos parâmetros de discriminação, ou seja, 100\% e 96,6\% dos intervalos cobriram o valor nulo, quando considerados os modelos 1d2PPN e 1d2PPSn, respectivamente. No que diz respeito aos traços latentes dos respondentes cerca de $93 \%$ e 97,25\% dos intervalos de credibilidade combriram o verdadeiro valor quando adotados os modelos 1d2PPN e 1d2PPSn, respectivamente.

A partir do gráfico (d) da Figura 5.3 é possível observar que os respondentes com traços latentes inferiores a -2 tiveram suas estimativas médias praticamente categorizadas para o menor valor estimado que foi aproximadamente -3 (e -4 quando obtidas sob o modelo 1d2PPSn). Uma explicação para este comportamento deve-se ao fato do teste não ter sido contemplado por itens cujos níveis de dificuldade representassem bem a região inferior a -2.2 da escala dos traços latentes. Este problema, portanto, independe do modelo adotado para se obter as estimativas mas sim da capacidade de se construir um teste com itens sob os mais variados níveis de dificuldade (e discriminação) de modo que as várias regiões da escala dos traços latentes sejam adequadamente contempladas. Ainda assim, devemos destacar que o modelo 1d2PPSn demonstrou-se mais flexível do que o 1d2PPN no sentido de permitir que valores inferiores a -3 fossem estimados para os traços latentes.

Nos histogramas da Figura 5.4 encontram-se algumas estatísticas descritivas das médias obtidas a partir de uma amostra da distribuição a posteriori dos traços latentes ex-

Matos, G. S.

$\mathrm{IME} / \mathrm{USP}$ 


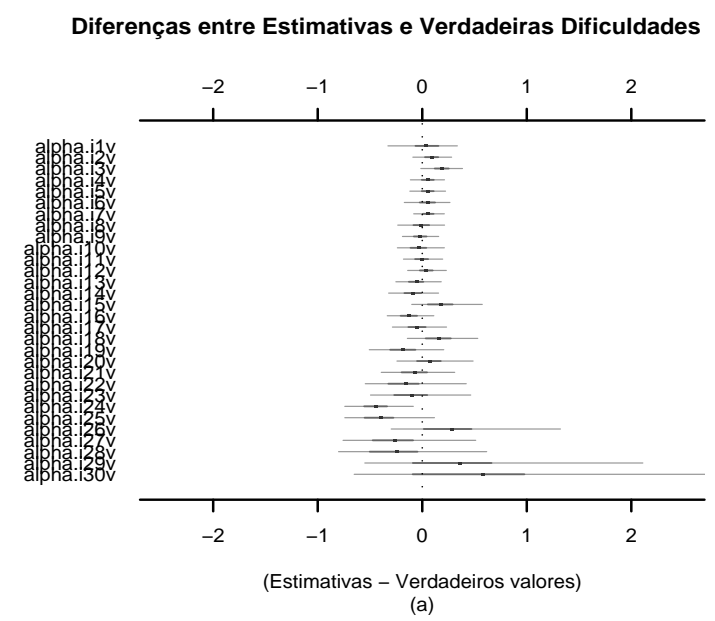

Diferenças entre Estimativas e Verdadeiros Traços Latentes

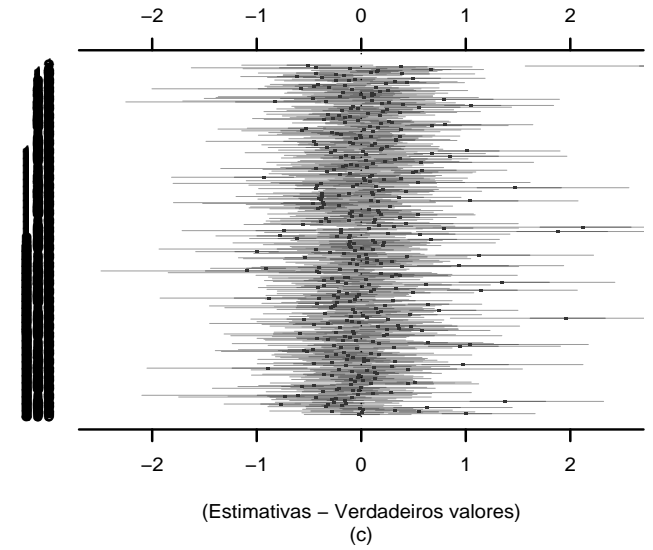

Diferenças entre Estimativas e Verdadeiras Discriminações

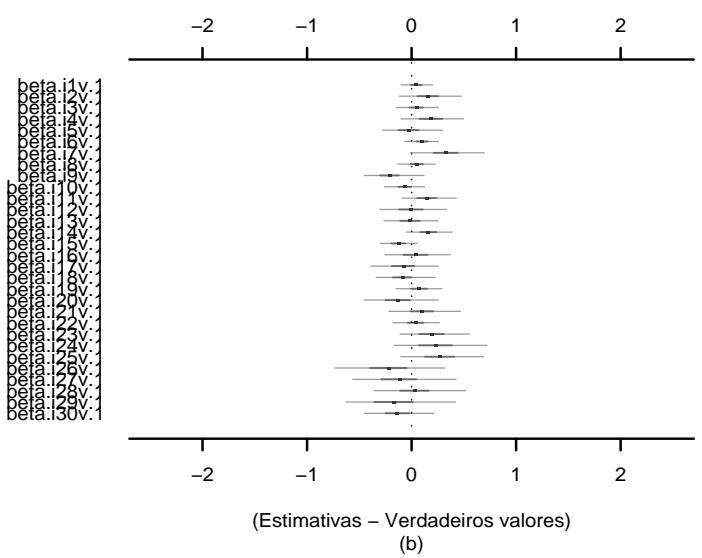

Verdadeiros Traços Latentes versus Estimativas

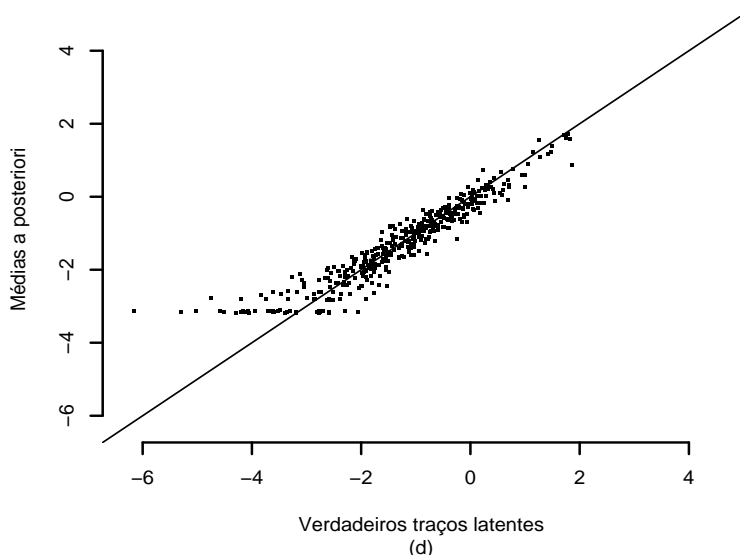

(d)

Figura 5.3 (a), (b) e (c) Intervalos quantílicos de credibilidade das diferenças entre estimativas obtidas a partir de uma amostra da distribuição a posteriori do modelo 1d2PPN e verdadeiros parâmetros do modelo 1d2PPSn. (d) Gráfico de dispersão entre verdadeiros traços latentes e médias amostrais da distribuição a posteriori sob o modelo 1d2PPN.

pressas na métrica dos verdadeiros parâmetros ${ }^{\dagger}$. A partir desta figura observamos que os parâmetros média e desvio padrão da verdadeira distribuição dos traços latentes são muito bem estimados, tanto pelo modelo $1 \mathrm{~d} 2 \mathrm{PPN}$ quanto $1 \mathrm{~d} 2 \mathrm{PPSn}$ (veja verdadeiros valores no Gráfico (c) da Figura 5.1). Com relação à verdadeira assimetria da amostra simulada dos traços latentes, igual a -0.42 , apenas a distribuição das estimativas obtidas pelo modelo 1d2PPSn foi que revelou um leve indício de assimetria negativa, $a_{3}=-0.17$. Este último resultado pode ser atribuído à maior flexibilidade na obtenção das estimativas quando

$\dagger$ As estimativas médias foram transformadas para a métrica dos verdadeiros traços latentes pelo método Média-Desvio, conforme discutido no último parágrafo da Seção 5.2.

Matos, G. S.

$\mathrm{IME} / \mathrm{USP}$ 
uma distribuição a priori assimétrica para os traços latentes é considerada. Ocorre, de fato, que sob o modelo 1d2PPSn o parâmetro populacional de assimetria pode ser diretamente estimado por sua distribuição a posteriori (veja densidade estimada no Gráfico (a) da Figura 5.5) e, conforme pode ser observado no Gráfico (b) da Figura 5.5, a estimação deste parâmetro ocorreu de modo satisfatório pois observamos que a média amostral da distribuição a posteriori estimada foi -1.41 e até mesmo um intervalo de credibilidade de 50\%; representado pelo "box" de menor dimensão; contém o verdadeiro parâmetro de assimetria que encontra-se representado pelo círculo não central.

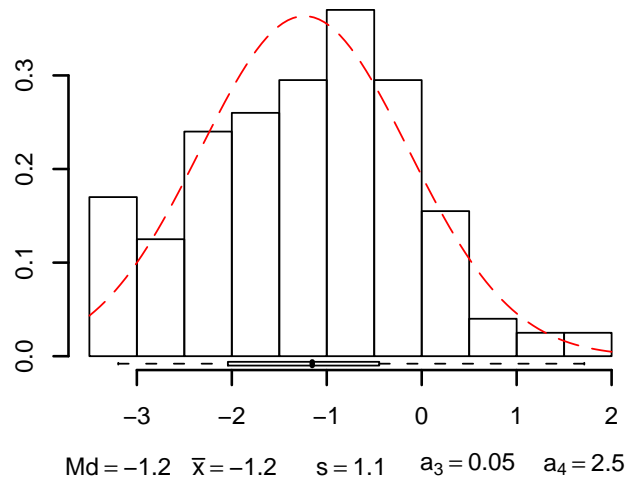

(a)

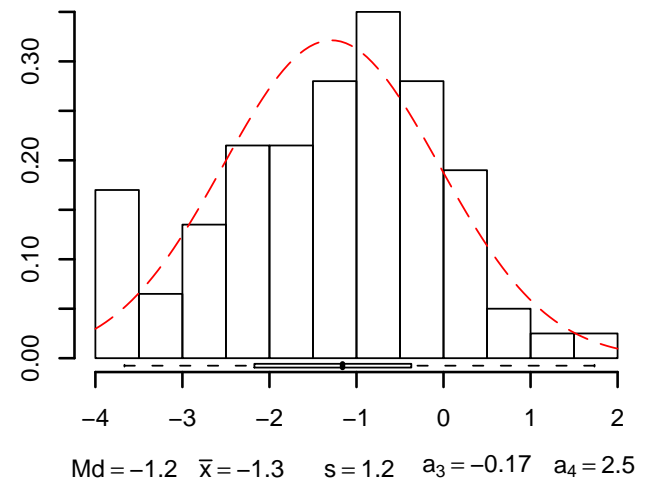

(b)

Figura 5.4 Histograma das estimativas médias dos traços latentes amostrados da distribuição a posterori expressas na métrica dos verdadeiros parâmetros: (a) Sob o modelo 1d2PPN (b) Sob o modelo 1d2PPSn.

\subsubsection{Análise das Estimativas Obtidas sob as Métricas Induzidas pelos Modelos 1d2PPN e 1d2PPSn}

Na Figura 5.6 apresentamos novamente alguns histogramas com estatísticas descritivas das médias obtidas a partir de uma amostra da distribuição a posteriori dos traços latentes, só que expressas na métrica original das estimativas obtidas por cada um dos modelos, ou seja, expressas na métrica correspondente aos valores gerados pelo algoritmo DAGS e sem equalização alguma. A partir desta figura observamos que as médias das estimativas obtidas pelos modelos 1d2PPN e 1d2PPSn são distintas e isto, equivocadamente, pode nos levar à interpretações distintas sobre o comportamento da distribuição dos traços latentes populacionais. Por exemplo, ao considerarmos o modelo 1d2PPSn podemos chegar à conclusão de que a habilidade média dos respondentes, igual a -1.1, é baixa quando comparada a um valor de referência (localização) igual a zero. De fato, esta conclusão é

Matos, G. S.

IME/USP 
Density of Skew posterior

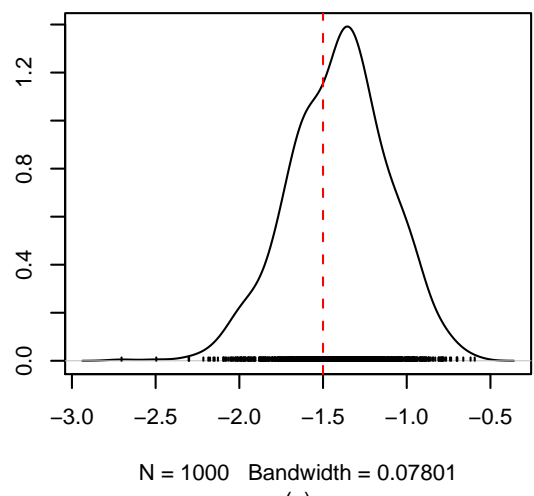

(a)
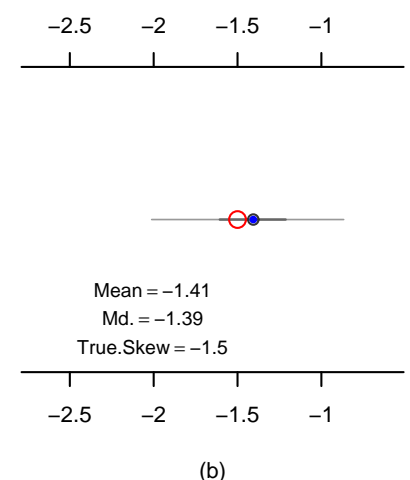

(b)

Figura 5.5 (a) Densidade a posteriori do parâmetro de assimetria $\delta$ (linha vertical localiza o valor da verdadeira assimetria) (b) Intervalos quantilicos de $50 \%$ e $95 \%$ de credibilidade do parâmetro de assimetria $\delta$.

confirmada quando observamos que a dificuldade média estimada para o teste foi igual a 0.15 (ver na Tabela 5.2). Por outro lado, ao considerarmos o modelo $1 \mathrm{~d} 2 \mathrm{PPN}$ e o valor zero como referência (localização), poderíamos concluir que a habilidade média dos respondentes igual a -0.055 é mediana. Acontece que tal conclusão não pode ser aceita sem que a dificuldade média estimada para o teste seja conhecida. A partir Tabela 5.2 é possível verificar, por exemplo, que a dificuldade média estimada pelo modelo 1d2PPN para o teste foi igual a 1.01 de maneira que a correta interpretação dos resultados deve nos levar à conclusão de que a habilidade média dos respondentes é baixa quando comparada a um valor de referência (localização) igual a um. Segue, portanto, que ambos modelos levam à conclusão análoga de que o conhecimento ou habilidade média dos respondentes está aquém do nível de dificuldade médio do teste numa magnitude aproximada de uma unidade da métrica induzida pelo modelo correspondente. Este resultado, conforme descreveremos em seguida, vai de encontro com as características do verdadeiro teste simulado.

A partir do Gráfico (b) da Figura 5.6 (ver também Gráfico (c) da Figura 5.1) e de uma breve comparação entre as estimativas dos parâmetros dos itens obtidas pelo modelo 1d2PPSn e os verdadeiros valores constantes na Tabela 5.2 é possível perceber que o modelo 1d2PPSn foi capaz de recuperar de modo satisfatório a verdadeira métrica dos parâmetros pois todas estimativas ficaram bem próximas dos verdadeiros valores sem a necessidade da equalização mencionada no último parágrafo da Seção 5.2. Por outro lado, a partir do Gráfico (a) da Figura 5.6, Gráfico (c) da Figura 5.1 e Tabela 5.2, o que se observa com relação às estimativas obtidas pelo modelo $1 \mathrm{~d} 2 \mathrm{PPN}$ é a ocorrência da Propriedade da Invariância da TRI a qual diz que as estimativas dos traços latentes (dos itens) são invariantes ao conjunto de itens (de respondentes) a que são submetidos. Isto quer dizer

Matos, G. S.

IME/USP 
estimativas médias dos traços latentes amostrados da distribuição a posterori

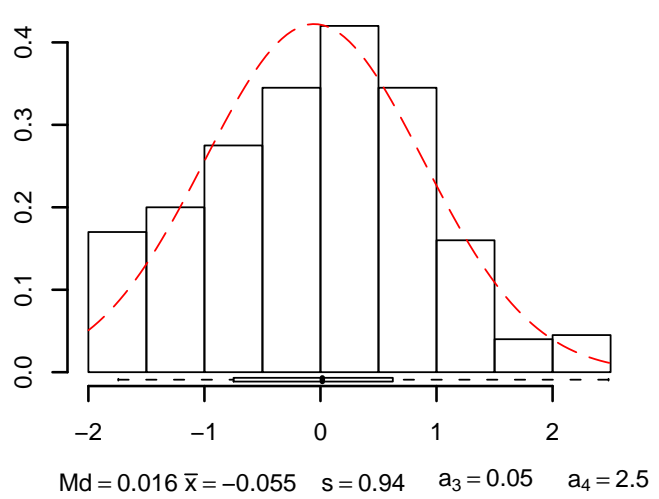

(a)

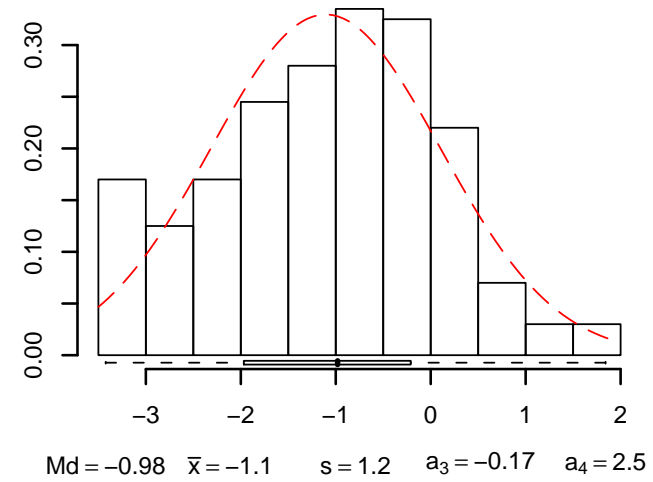

(b)

Figura 5.6 Histograma das estimativas médias dos traços latentes amostrados da distribuição a posterori expressas na métrica das estimativas originais: (a) Sob o modelo 1d2PPN (b) Sob o modelo 1d2PPSn.

que, a menos de flutuações aleatórias, as probabilidades de resposta aos itens mantém-se invariantes ao conjunto de itens e/ou respondentes avaliados (Andrade et al. (2000, pág. 15 e 88), Hambleton et al. (1991)). Para melhor compreender a ocorrência desta propriedade em nossa análise, aproveitamos o fato de estarmos analisando um conjunto de dados simulados e observamos que ao considerar a distribuição $\operatorname{Sn}(0,1, \delta) \operatorname{com} \delta=-1.5$ para os traços latentes, induzimos um valor esperado igual a -1.19 de modo que a média amostral dos traços latentes simulados resultou no valor igual a -1.3 (Gráfico (c) da Figura 5.1). Deste modo, o teste préviamente definido para conter itens com níveis de dificuldade entre fácil (-2.2), mediano (0.0) e difícil (2.2) (ver Tabela 5.1) passou a conter, de fato, itens difíceis para a particular população de respondentes e isto, no caso do modelo $1 \mathrm{~d} 2 \mathrm{PPN}$, provocou a obtenção de estimativas positivamente viesadas e quase que sistemática de magnitude aproximada de uma unidade, tanto para os parâmetros de dificuldade quanto para os traços latentes. Em termos práticos, observamos que ao adotar uma distribuição a priori normal padrão para os traços latentes, estabelecemos uma determinada escala de medida (ou métrica) a qual até então era arbitrária e, ao final, os itens são estimados como sendo uma unidade mais difícil quando comparados aos verdadeiros níveis de dificuldade. Mas os traços latentes estimados, quando comparados aos verdadeiros, também refletem um deslocamento à direita por uma unidade, representando, assim, maiores níveis de habilidade e por conta disso tais estimativas levam à probabilidades equivalentes às calculadas sob a métrica do modelo 1d2PPSn; assim como, a menos de erros aleatórios, sob a métrica induzida pelos verdadeiros parâmetros. Para ilustrar a veracidade de tal conclusão, apresentamos na Figura 5.7 a relação entre as probabilidades calculadas sob as 
três métricas envolvidas na presente análise: a dos parâmetros originais, a induzida pelo modelo 1d2PPN e 1d2PPSn. Para finalizar, destacamos que tais observações nos chama atenção para o fato de que, para interpretar as estimativas obtidas por um determinado modelo, seja com distribuição a priori simétrica ou assimétrica para os traços latentes, é importante sempre levar em consideração as relações de ordem e magnitude entre a média das estimativas médias dos traços latentes e a média das dificuldades estimadas dos itens.

Matos, G. S. 
Tabela 5.2 Verdadeiros parâmetros dos itens e médias amostrais da distribuição a posteriori segundo modelos 1d2PPN e 1d2PPSn.

\begin{tabular}{|c|c|c|c|c|c|c|}
\hline \multirow[b]{2}{*}{ Item } & \multicolumn{3}{|c|}{ Dificuldade } & \multicolumn{3}{|c|}{ Discriminação } \\
\hline & Verd. & $1 \mathrm{~d} 2 \mathrm{PPN}$ & 1d2PPSn & Verd. & $1 \mathrm{~d} 2 \mathrm{PPN}$ & $1 d 2 P P S n$ \\
\hline $\mathrm{i} 1 \mathrm{v}$ & -2.20 & -0.85 & -2.11 & 0.55 & 0.69 & 0.54 \\
\hline $\mathrm{i} 2 \mathrm{v}$ & -2.02 & -0.65 & -1.84 & 1.16 & 1.54 & 1.19 \\
\hline $\mathrm{i} 3 \mathrm{v}$ & -1.85 & -0.42 & -1.55 & 0.89 & 1.09 & 0.84 \\
\hline $\mathrm{i} 4 \mathrm{v}$ & -1.68 & -0.39 & -1.50 & 1.26 & 1.68 & 1.31 \\
\hline $\mathrm{i} 5 \mathrm{v}$ & -1.51 & -0.24 & -1.31 & 1.42 & 1.63 & 1.29 \\
\hline$i 6 v$ & -1.34 & -0.10 & -1.14 & 0.63 & 0.85 & 0.68 \\
\hline $\mathrm{i} 7 \mathrm{v}$ & -1.17 & 0.05 & -0.93 & 1.28 & 1.87 & 1.52 \\
\hline $\mathrm{i} 8 \mathrm{v}$ & -1.00 & 0.14 & -0.84 & 0.69 & 0.86 & 0.69 \\
\hline $\mathrm{i} 9 \mathrm{v}$ & -0.80 & 0.30 & -0.62 & 1.44 & 1.43 & 1.17 \\
\hline $\mathrm{i} 10 \mathrm{v}$ & -0.67 & 0.40 & -0.50 & 0.90 & 0.97 & 0.78 \\
\hline $\mathrm{i} 11 \mathrm{v}$ & -0.55 & 0.53 & -0.34 & 1.00 & 1.34 & 1.09 \\
\hline $\mathrm{i} 12 \mathrm{v}$ & -0.43 & 0.67 & -0.17 & 1.38 & 1.60 & 1.34 \\
\hline $\mathrm{i} 13 \mathrm{v}$ & -0.30 & 0.70 & -0.14 & 1.13 & 1.30 & 1.09 \\
\hline $\mathrm{i} 14 \mathrm{v}$ & -0.18 & 0.78 & -0.04 & 0.78 & 1.09 & 0.91 \\
\hline $\mathrm{i} 15 \mathrm{v}$ & -0.06 & 1.11 & 0.34 & 0.82 & 0.81 & 0.67 \\
\hline $\mathrm{i} 16 \mathrm{v}$ & 0.06 & 0.95 & 0.16 & 1.23 & 1.48 & 1.26 \\
\hline $\mathrm{i} 17 \mathrm{v}$ & 0.18 & 1.12 & 0.35 & 1.33 & 1.46 & 1.27 \\
\hline i18v & 0.30 & 1.41 & 0.68 & 1.07 & 1.14 & 0.99 \\
\hline $\mathrm{i} 19 \mathrm{v}$ & 0.43 & 1.22 & 0.48 & 0.74 & 0.94 & 0.79 \\
\hline $\mathrm{i} 20 \mathrm{v}$ & 0.55 & 1.55 & 0.81 & 1.28 & 1.34 & 1.22 \\
\hline $\mathrm{i} 21 \mathrm{v}$ & 0.67 & 1.52 & 0.80 & 1.05 & 1.34 & 1.18 \\
\hline $\mathrm{i} 22 \mathrm{v}$ & 0.80 & 1.56 & 0.86 & 0.71 & 0.87 & 0.74 \\
\hline $\mathrm{i} 23 \mathrm{v}$ & 1.00 & 1.79 & 1.07 & 0.84 & 1.20 & 1.09 \\
\hline $\mathrm{i} 24 \mathrm{v}$ & 1.17 & 1.64 & 0.89 & 1.21 & 1.68 & 1.61 \\
\hline $\mathrm{i} 25 \mathrm{v}$ & 1.34 & 1.82 & 1.11 & 0.98 & 1.46 & 1.37 \\
\hline $\mathrm{i} 26 \mathrm{v}$ & 1.51 & 2.56 & 1.80 & 1.42 & 1.40 & 1.47 \\
\hline $\mathrm{i} 27 \mathrm{v}$ & 1.68 & 2.23 & 1.48 & 1.32 & 1.40 & 1.43 \\
\hline $\mathrm{i} 28 \mathrm{v}$ & 1.85 & 2.39 & 1.68 & 1.05 & 1.26 & 1.23 \\
\hline $\mathrm{i} 29 \mathrm{v}$ & 2.02 & 3.06 & 2.23 & 1.18 & 1.18 & 1.30 \\
\hline $\mathrm{i} 30 \mathrm{v}$ & 2.20 & 3.40 & 2.78 & 0.80 & 0.77 & 0.72 \\
\hline $\min$. & -2.20 & -0.85 & -2.11 & 0.55 & 0.69 & 0.54 \\
\hline média & 0.00 & 1.01 & 0.15 & 1.05 & 1.26 & 1.09 \\
\hline mediana & 0.00 & 1.03 & 0.25 & 1.06 & 1.32 & 1.18 \\
\hline $\max$ & 2.20 & 3.40 & 2.78 & 1.44 & 1.87 & 1.61 \\
\hline
\end{tabular}

Matos, G. S. 

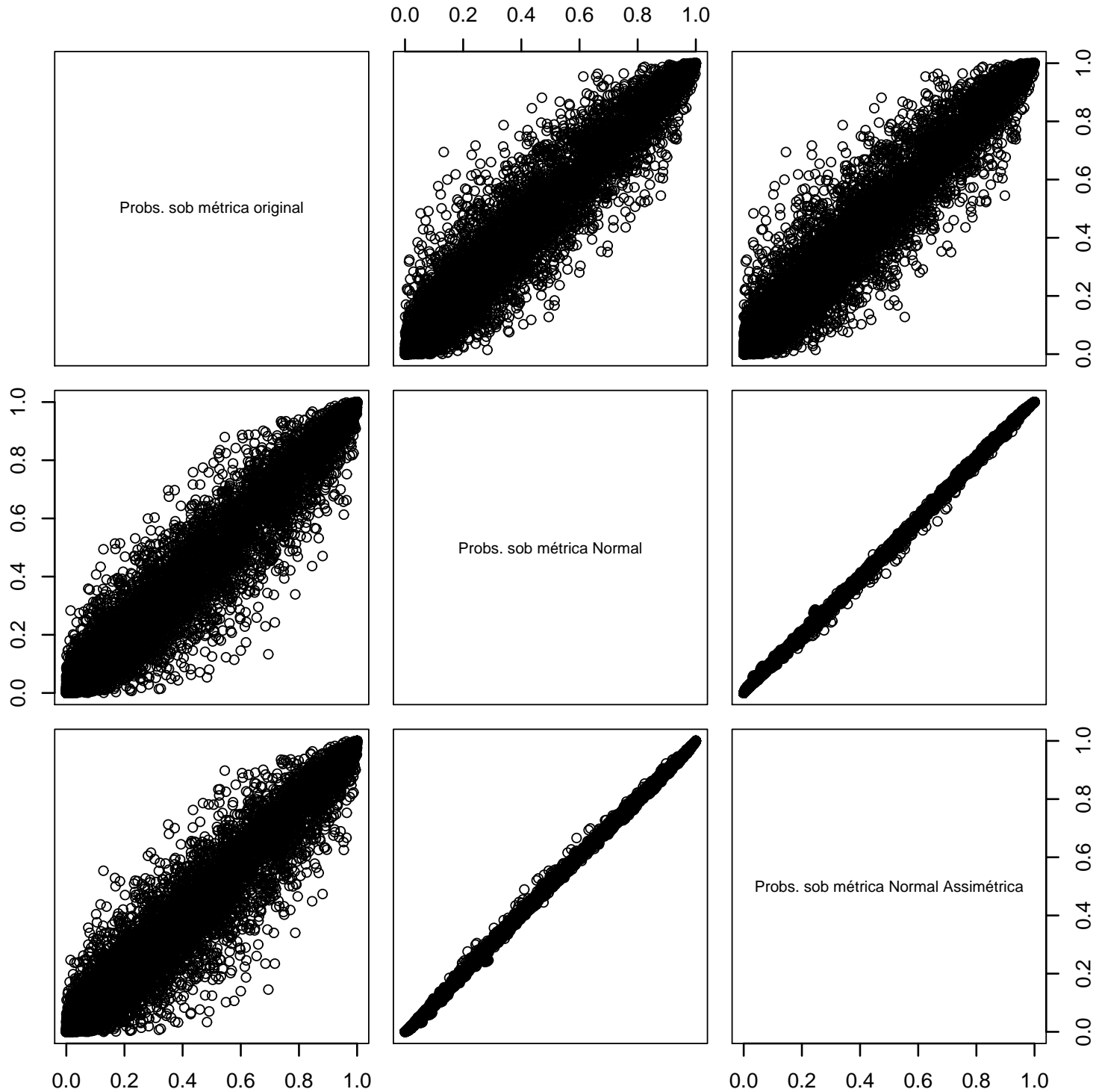

Figura 5.7 Gráficos de dispersão entre as probabilidades de resposta correta ao item calculadas sob as três métricas envolvidas na presente análise: dos parâmetros originais, induzida pelo modelo 1d2PPN e 1d2PPSn.

Matos, G. S. 


\subsubsection{Seleção de Modelo}

O principal objetivo desta seção é avaliar o desempenho dos modelos 1d2PPN e 1d2PPSn através de algumas medidas que não exijam o conhecimento dos verdadeiros parâmetros geradores do conjunto de dados. Deste modo, os procedimentos aqui considerados são de grande utilidade para o caso de uma análise de dados reais.

Na Tabela 5.3 apresentamos algumas medidas comumente utilizadas na avaliação de ajuste e seleção de modelos bayesianos; tais como: Critério de Informação do Desvio (DIC; Spiegelhalter et al. (2002)), Esperança da Informação de Akaike (EAIC; (Bazán, 2005; Brooks, 2002)), Esperança da Informação Bayesiana (EBIC; (Bazán, 2005; Brooks, 2002)) e Pseudo Fator de Bayes (PsBF; Sahu (2002), Gelfand (1996)).

Tabela 5.3 Medidas de critério de ajuste e seleção de modelos.

\begin{tabular}{lcccc}
\hline \multirow{2}{*}{ Modelo } & \multicolumn{3}{c}{ Critério de Ajuste } & \multirow{2}{*}{ Critério de Seleção } \\
\cline { 2 - 5 } & DIC $\left(p_{D}{ }^{*}\right)$ & EAIC & EBIC & PsBF \\
\hline 1d2PPN & $7596.13(410.41)$ & 8105.71 & 11506.34 & 0.103 \\
1d2PPSn & $7577.88(414.03)$ & 8085.86 & 11493.87 & 9.709 \\
\hline \hline
\end{tabular}

* Quantidade que mede a complexidade ou o número efetivo de parâmetros do modelo.

Como podemos observar, o DIC, EAIC e EBIC fornecem evidências favoráveis ao modelo 1d2PPSn quando comparado ao modelo $1 \mathrm{~d} 2 \mathrm{PPN}$ pois os valores correspondentes ao modelo 1d2PPSn são ligeiramente menores, indicando, portanto, que este modelo é o que melhor se ajusta aos dados. O valor do PsBF igual a 9.709 (=1/0.103); conforme tabela calibrada por Raftery (1996) para o Fator de Bayes; nos fornece uma evidência relativamente ${ }^{\ddagger}$ forte a favor do ajuste e seleção do modelo 1d2PPSn.

\subsubsection{Considerações Adicionais e Conclusão}

Nas últimas seções, em um modelo unidimensional da TRI, procuramos analisar possíveis vantagens com relação à adoção de uma distribuição normal assimétrica para a priori dos traços latentes quando, de fato, a verdadeira distribuição é normal assimétrica com um nível de assimetria moderado (Seção 5.2.1). Na Seção 5.2.4 foi possível observar que independente da distribuição a priori para os traços latentes ser normal simétrica ou assimétrica; as estimativas recuperam de forma satisfatória os verdadeiros parâmetros do modelo gerador dos dados. Apesar da similaridade entre as estimativas obtidas pelos

‡ Esta medida; diferentemente do Fator de Bayes; não possui uma interpretação tão óbvia pois a mesma não surgiu formalmente como uma razão de chances (Gelfand, 1996).

Matos, G. S.

IME/USP 
modelos 1d2PPN e 1d2PPSn, foi possível observar que o modelo 1d2PPSn comportou-se de forma mais flexível pois além de corretamente ter fornecido indícios quanto a uma assimetria negativa e moderada também forneceu indícios de que os verdadeiros traços latentes com valores inferiores à -2.2 podem ser melhor estimados. Esta flexibilidade é a provável explicação para o fato de medidas tais como o DIC, EAIC e EBIC indicarem o modelo 1d2PPSn como o que melhor se ajusta aos dados (Seção 5.2.6).

Na Seção 5.2.5 foi possível observar uma questão de grande importância prática para a análise e interpretação das estimativas obtidas pelo modelo 1d2PPN e 1d2PPSn. Naquela seção, observamos que os modelos geram estimativas distintas pois métricas distintas são induzidas, dependendo da especificação de uma distribuição a priori simétrica ou assimétrica para os traços latentes. Apesar disto, conclusões e interpretações quanto às verdadeiras características de um teste podem ser corretamente e analogamente obtidas desde que as relações de ordem e magnitude entre a média das estimativas médias dos traços latentes e a média das dificuldades estimadas dos itens sejam levadas em consideração.

Conforme é possível perceber nas análises desenvolvidas nesta seção, em termos de probabilidades estimadas de resposta aos itens, não há vantagem alguma em adotar uma distribuição a priori para os traços latentes normal assimétrica com o intuito de estimar os parâmetros do modelo 1-dimensional de 2 Parâmetros Probito da TRI (1d2PP), mesmo quando a verdadeira distribuição dos traços latentes é moderamente assimétrica. Apesar disto, se levarmos em consideração que o foco da análise de um conjunto de respostas aos itens concentra-se nas características dos itens e traços latentes e que as estimativas obtidas pelo modelo 1d2PPSn demonstraram-se mais flexíveis no sentido de melhor representar os verdadeiros valores dos parâmetros populacionais e, se além disso, levarmos em consideração o fato de que medidas de ajuste e seleção de modelos tais como DIC, EAIC, EBIC e PsBF revelaram-se favoráveis a escolha do modelo 1d2PPSn; é possível concluir que existe sim alguma melhoria e vantagem em se adotar o modelo 1d2PPSn para a análise e a interpretação dos dados simulados nesta seção. Vale lembrar, ainda, que o uso do modelo 1d2PPSn e a estimação bayesiana via método DAGS revelou-se eficaz para a estimação da verdadeira forma da distribuição dos traços latentes, conforme podemos observar através do Gráfico (c) da Figura 5.1 e Figuras 5.4, 5.5 e 5.6. Análises como as descritas nesta seção com assimetria positiva para a verdadeira distribuição dos traços latentes resultaram em conclusões análogas.

Matos, G. S.

$\mathrm{IME} / \mathrm{USP}$ 


\subsection{Dados Simulados 2: Teste Bidimensional}

Nesta seção um teste (simulado) do tipo bidimensional (Seção 3.2.1) será analisado e de modo semelhante à análise desenvolvida na seção anterior o principal objetivo será, além de obter uma impressão sobre a eficácia do método de estimação DAGS, investigar sobre possíveis vantagens e/ou desvantagens em se adotar uma distribuição normal multivariada assimétrica como informação a priori sobre o comportamento dos traços latentes de uma determinada população. Com este intuito um teste composto por 30 itens foi idealizado de maneira similar ao descrito na Sub-seção 5.2. As características do teste simulado/idealizado são detalhadamente descritas na próxima sub-seção.

\subsubsection{Características dos Verdadeiros Parâmetros dos Itens e Tra- ços Latentes}

Na Tabela 5.4 constam os verdadeiros parâmetros dos itens segundo um modelo bidimensional de 2 Parâmetros Probito (2d2PP; Seção 3.2.1). Nesta Tabela, observamos que os parâmetros associados à discriminação dos itens na segunda (primeira) dimensão que compoem os primeiros (últimos) 15 itens assumem valores relativamente menores e próximos a zero quando comparados com os correspondentes da outra dimensão. Em termos prático, um conjunto de itens com tais características representa um teste bidimensional com estrutura aproximadamente simples e isto significa que embora múltiplos traços latentes sejam necessários para se obter uma resposta a um item, apenas um dos traços latentes é substancialmente necessário (McDonald, 1999). Outro aspecto prático e de interesse para a interpretação dos parâmetros dos itens diz que, devido a multidimensionalidade, os valores dos parâmetros de "dificuldade" (intercepto $\beta_{1 j}$ ) e "discriminação" (inclinação $\beta_{2 j 1}$ e $\beta_{2 j 2}$ ) de um item não mais podem ser diretamente interpretados como tais, apesar dos mesmos estarem relacionados ${ }^{\S}$. Na análise do presente conjunto de dados tais interpretações ainda serão consideradas, principalmente por estarmos diante de sub-testes aproximadamente unidimensionais. Quantidades que verdadeiramente podem ser interpretadas como sendo o nível de dificuldade e a discriminação de um item multidimensional são rigorosamente definidas por Reckase (1997) (ver também Nojosa (2002)) e diante das considerações aqui descritas avisamos que nos referiremos aos 15 primeiros itens como pertencentes a um sub-teste 1 aos demais a um sub-teste 2 .

Para a verdadeira distribuição dos traços será considerada uma distribuição normal assimétrica bivariada com vetor de localização $\boldsymbol{\mu}_{\theta}=(-0.5,1.7)^{\prime}$, matrix de escala $\Sigma_{\theta}=\left(\begin{array}{ll}0.90 & 0.65 \\ 0.65 & 0.70\end{array}\right)$ e vetor associado à assimetria $\boldsymbol{\delta}=(0,-1.5)^{\prime}$. Os valores destes

$\S$ Veja no terceiro parágrafo da Sub-seção 5.2 que situação análoga ocorre no caso de um modelo de resposta ao item unidimensional.

Matos, G. S.

IME/USP 
Tabela 5.4 Parâmetros dos itens (bidimensionais) utilizados para gerar os dados dicotômicos.

\begin{tabular}{lrrrrrrr}
\hline \hline & \multicolumn{3}{c}{ Parâmetros } & & \multicolumn{3}{c}{ Parâmetros } \\
\cline { 2 - 8 } Itens do Sub-teste 1 & $\beta_{1 j}$ & $\beta_{2 j 1}$ & $\beta_{2 j 2}$ & Itens do Sub-teste 2 & $\beta_{1 j}$ & $\beta_{2 j 1}$ & $\beta_{2 j 2}$ \\
\hline i1t1 & -2.20 & 0.55 & 0.03 & i1t2 & -2.02 & 0.33 & 1.16 \\
i2t1 & -1.68 & 1.26 & 0.19 & i2t2 & -1.85 & 0.34 & 0.89 \\
i3t1 & -1.51 & 1.42 & 0.37 & i3t2 & -1.34 & 0.06 & 0.63 \\
i4t1 & -1.17 & 1.28 & 0.29 & i4t2 & -1.00 & 0.08 & 0.69 \\
i5t1 & -0.80 & 1.44 & 0.26 & i5t2 & -0.67 & 0.10 & 0.90 \\
i6t1 & -0.55 & 1.00 & 0.33 & i6t2 & -0.43 & 0.34 & 1.38 \\
i7t1 & -0.30 & 1.13 & 0.27 & itt2 & -0.18 & 0.03 & 0.78 \\
i8t1 & -0.06 & 0.82 & 0.06 & i8t2 & 0.06 & 0.34 & 1.23 \\
i9t1 & 0.18 & 1.33 & 0.21 & i9t2 & 0.30 & 0.37 & 1.07 \\
i10t1 & 0.43 & 0.74 & 0.20 & i10t2 & 0.55 & 0.24 & 1.28 \\
i11t1 & 0.67 & 1.05 & 0.37 & i11t2 & 0.80 & 0.02 & 0.71 \\
i12t1 & 1.00 & 0.84 & 0.13 & i12t2 & 1.17 & 0.28 & 1.21 \\
i13t1 & 1.34 & 0.98 & 0.34 & i13t2 & 1.51 & 0.20 & 1.42 \\
i14t1 & 1.68 & 1.32 & 0.35 & i14t2 & 1.85 & 0.11 & 1.05 \\
i15t1 & 2.02 & 1.18 & 0.17 & i15t2 & 2.20 & 0.22 & 0.80 \\
\hline \hline
\end{tabular}

parâmetros resultam no valor esperado $E(\boldsymbol{\theta})=(-0.50,0.50)^{\prime}$, na matriz de covariâncias $\operatorname{Cov}(\boldsymbol{\theta})=\left(\begin{array}{ll}0.90 & 0.65 \\ 0.65 & 1.52\end{array}\right)$ e na matriz de correlações $\operatorname{Cor}(\boldsymbol{\theta})=\left(\begin{array}{cc}1 & 0.56 \\ 0.56 & 1\end{array}\right)$. Todas estas características são obtidas das seguintes expressões encontradas em Sahu et al. (2003):

$$
E(\boldsymbol{\theta})=\boldsymbol{\mu}_{\theta}+\left(\frac{2}{\pi}\right)^{\frac{1}{2}} \boldsymbol{\delta} \quad \text { e } \quad \operatorname{Cov}(\boldsymbol{\theta})=\boldsymbol{\Sigma}_{\theta}+\left(1-\frac{2}{\pi}\right) \boldsymbol{D}^{2}
$$

em que $\boldsymbol{D}=\operatorname{diag}\left\{\delta_{1}, \ldots, \delta_{K}\right\}$ é uma matriz diagonal com parâmetros $\delta_{1}, \ldots, \delta_{k}, \ldots, \delta_{K}$ associados à assimetria (forma) da distribuição assimétrica multivariada na $k$-ésima dimensão, $k=1, \ldots, K$.

A escolha destes parâmetros tem a intenção de simular uma população de respondentes cujo comportamento dos traços latentes na primeira dimensão possa ser representada por uma distribuição nomal simétrica com o valor do parâmetro de localização aproximadamente inferior ao nível médio de dificuldade do sub-teste 1, simulando assim um sub-teste relativamente difícil. Note que isto reproduz de maneira semelhante a situação do teste unidimensional simulado na Seção 5.2 sendo que, no presente caso, há ausência de assimetria. A idéia para o comportamento da distribuição dos traços latentes na se- 
gunda dimensão é de que a mesma possa ser representada por uma distribuição normal assimétrica com um nível de assimetria negativo moderado; assim como foi a assimetria considerada na Seção 5.2. Além disso, a adoção do valor positivo e igual a 1.7 para o parâmetro de localização na segunda dimensão da distribuição dos traços latentes teve por objetivo obter o valor esperado igual a 0.5 nesta dimensão. Fazendo desta forma, o sub-teste 2 pode ser considerado como sendo relativamente fácil, uma vez que o nível médio de dificuldade deste é 0.0633 (média dos parâmetros $\beta_{1 j}$ deste sub-teste; Tabela 5.4). Finalmente, a escolha dos parâmetros da matriz de escala teve como propósito a obtenção de uma correlação moderada e positiva entre as duas dimensões dos traços latentes.

Na Tabela 5.5 apresentamos algumas características de uma amostra de tamanho $N=800$ da distribuição dos traços latentes acima descrita e, conforme já mencionado, os valores desta amostra juntamente com os parâmetros dos itens da Tabela 5.4 formam a base necessária para a geração do conjunto de dados dicotômicos segundo um modelo 2d2PPSn.

Tabela 5.5 Estatísticas descritivas da amostra bidimensional dos traços latentes utilizada na geração dos dados dicotômicos.

\begin{tabular}{cccccccccc}
\hline \hline & Mín. & Q1 & Md & Q3 & Máx. & $\bar{\theta}$ & $s^{2}$ & $a_{3}$ & $r_{12}$ \\
\hline$\theta_{1}$ & -3.390 & -1.171 & -0.533 & 0.178 & 2.455 & -0.512 & 0.973 & -0.018 & 0.557 \\
$\theta_{2}$ & -3.901 & -0.330 & 0.637 & 1.431 & 4.044 & 0.513 & 1.625 & -0.350 & - \\
\hline \hline
\end{tabular}

Notação: Q1 e Q3: 1ํㅡ e $3 \underline{0}$ Quartis, Md: mediana, $\bar{\theta}$ : média amostral, $s^{2}$ : variância amostral, $a_{3}$ : assimetria amostral e $r_{12}$ : correlação amostral entre os traços latentes da dimensão 1 e 2.

\subsubsection{Modelos e Metodologia para as Análises}

A análise do atual conjunto de dados simulado será conduzida em um primeiro momento sob o modelo 2d2PP (Seção 3.2) com distribuições padrão normal e normal assimétrica para os traços latentes. Neste momento, o interesse maior será analisar a qualidade das inferências considerando-se somente a estimação dos parâmetros dos itens e traços latentes sem considerar a estimação dos hiperparâmetros de localização e escala da distribuição dos traços latentes. Após esta etapa inicial, as análises serão conduzidas sob o modelo 2m2PP (Seção 3.2.2) com distribuições normal e normal assimétrica para os traços latentes e neste momento os hiperparâmetros de localização e escala da distribuição dos traços latentes também serão estimados. É importante ressaltar que para as análises do primeiro momento nenhum dos parâmetros dos itens serão fixados de modo que uma análise do tipo exploratória será realizada no sentido de identificar a estrutura da matrix de discriminação ("cargas fatoriais"); assim como é feito com o uso do software TESTFACT

Matos, G. S.

IME/USP 
Bock et al. (2003). De outra forma as análises sob os modelos 2m2PP assumirá um caráter confirmatório pois restrições de nulidade serão estabelecidas sobre alguns elementos da matrix de discriminação, $\boldsymbol{\beta}_{2}$, conforme o item pertença ao sub-teste 1 ou 2 (ver Seção 3.2.2). Eventualmente, em todos estes casos alguns dos parâmetros de interesse não serão estimados devido às restrições de identificabilidade (assunto discutido nas Sub-seções 3.3, 5.3.3.1 e 5.3.4.4).

As estimativas dos itens obtidas por cada um dos modelos acima descritos serão avaliadas através de um índice que denominaremos Raiz do Erro Quadrático Médio ( $R E Q M)$. Este índice permitirá avaliar as diferenças entre as Funções de Resposta ao Item (FRI's) verdadeiras e estimadas de um determinado item $j$ e sua expressão é dada por:

$$
R E Q M_{j}=\sqrt{\frac{1}{n_{j}} \sum_{i=1}^{n_{j}}\left[P\left(\boldsymbol{\theta}_{i} ; \widehat{\beta}_{1 j}, \widehat{\boldsymbol{\beta}}_{2 j}\right)-P\left(\boldsymbol{\theta}_{i} ; \beta_{1 j}, \boldsymbol{\beta}_{2 j}\right)\right]^{2}},
$$

em que $P\left(\boldsymbol{\theta}_{i} ; \beta_{1 j}, \boldsymbol{\beta}_{2 j}\right) \equiv \Phi\left(\boldsymbol{X}_{i}^{\prime} \boldsymbol{\beta}_{12 j}\right)$ é a probabilidade de resposta correta a um item obtida a partir do modelo $2 \mathrm{~d} 2 \mathrm{PP}$ (Seção 3.2) avaliado nos verdadeiros parâmetros dos itens e traços latentes. $P\left(\boldsymbol{\theta}_{i} ; \widehat{\beta}_{1 j}, \widehat{\boldsymbol{\beta}}_{2 j}\right)$ corresponde, portanto, ao modelo $2 \mathrm{~d} 2 \mathrm{PP}$ avaliado nas estimativas pontuais (médias amostrais da distribuição a posteriori) dos parâmetros dos itens e verdadeiros traços latentes. O somatório presente na expressão (5.2) percorre o conjunto dos respondentes que responderam ao $j$-ésimo item e $n_{j}$ representa o total destes respondentes.

Para uma comparação mais detalhada das estimativas obtidas pelos modelos $2 \mathrm{~d} 2 \mathrm{PPN}$ (e $2 \mathrm{~m} 2 \mathrm{PPN}$ ) e $2 \mathrm{~d} 2 \mathrm{PPSn}$ (e $2 \mathrm{~m} 2 \mathrm{PPSn}$ ), desvios absolutos entre estimativas e verdadeiros parâmetros serão considerados. Além disso, de modo análogo à análise da Seção 5.2, o DIC, EAIC, EBIC e PsBF serão utilizados como critérios de ajuste e seleção de modelos.

No que diz respeito às análises comparativas que desenvolveremos nesta seção é necessário chamarmos a atenção para o fato de que algumas medidas como a Raiz do Erro Quadrático Médio $(R E Q M)$ e os desvios absolutos entre estimativas e verdadeiros parâmetros são dependentes da escala de medida ou métrica dos parâmetros. Esta dependência, conforme De Ayala e Sava-Bolesta (1999) e experiência adquirida na análise do teste unidimensional desenvolvida na Seção5.2 do presente trabalho, torna essencial a transformação de localização, escala e rotação (equalização) das estimativas $\widehat{\beta}_{1 j}, \widehat{\boldsymbol{\beta}}_{2 j}$ e $\widehat{\theta}_{i}$ para a métrica dos verdadeiros parâmetros. Pois, sem isto, falsos vieses podem ser observados devido a não-comparabilidade entre a escala induzida por cada um dos modelos estimados e escala do verdadeiro modelo. Os métodos de equalização a posteriori (pós-obtenção das estimativas) de modelos multidimensionais na TRI ainda são pouco conhecidos e dentre algumas poucas propostas adotamos o método desenvolvido por Reckase e Martineau (2004) pois o mesmo; além de apresentar facilidade para a implementação computacional; não apresenta deficiências de métodos anteriores e independe de uma correta correspondência entre as colunas (dimensões) da matriz de discriminações estimadas e verdadeiras.

Matos, G. S.

$\mathrm{IME} / \mathrm{USP}$ 


\subsubsection{Informações e Resultados sob os modelos 2d2PPN e 2d2PPSn}

\subsubsection{Sobre as Prioris e a Identificabilidade dos Modelos}

As distribuições a priori dos parâmetros dos itens foram especificadas de modo análogo ao caso do modelo de resposta ao item unidimensional de 2 Parâmetros Probito (Sub-seção 5.2.2), sendo que a mesma especificação foi adotada para os hiperparâmetros da primeira e segunda dimensão da distribuição dos parâmetros de discriminação. A especificação das distribuições a priori dos vetores dos traços latentes também foi realizada de modo análogo ao caso unidimensional e, conforme já mencionado, distribuições normal bivariada simétrica e assimétrica padrão foram adotadas quando considerado o modelo $2 \mathrm{~d} 2 \mathrm{PPN}$ e 2d2PPSn, respectivamente. Para o parâmetro de assimetria deste último modelo, uma distribuição pouco informativa dada por $\boldsymbol{\delta} \sim N_{2}(\mathbf{0}$, diag $(100,100))$ foi adotada.

Com relação à falta de identificabilidade do modelo $2 \mathrm{~d} 2 \mathrm{PP}$, lembramos que a especificação de uma distribuição normal bivariada na forma padrão (simétrica ou assimétrica) define uma métrica para a escala dos traços latentes. Tal especificação, além de permitir que os parâmetros estimados sejam adequadamente analisados e interpretados, também possibilita que análises de convergência da Cadeia de Markov possam ser adequadamente realizadas pois uma distribuição na forma padrão delimita uma região de possíveis valores para os parâmetros do modelo que antes era arbitrária. Apesar disto, conforme discutido na Sub-seção 3.3.1.2, sabemos que no caso da distribuição normal bivariada simétrica, tal especificação não elimina a não-identificabilidade do modelo 2d2PP com relação à rotação dos traços latentes e/ou da matriz de discriminação. Sendo assim, dificuldades relacionadas às análises de convergência da Cadeia de Markov ainda podem ocorrer e por isso restrições adicionais e/ou especificações adequadas sobre as distribuições a priori dos parâmetros dos itens precisam ser cuidadosamente consideradas (ver Seções 3.3.1.2 e 3.3.2). Diante desta necessidade e sem querer restringir alguns parâmetros dos itens à valores fixos, as distribuições associadas aos parâmetros de discriminação foram todas truncadas à esquerda do zero e, por questões de uniformidade, tais distribuições truncadas também foram especificadas para o caso em que a distribuição normal multivariada é assimétrica. É interessante destacar que a decisão por não fixar alguns parâmetros de itens imita o procedimento padrão e inflexível disponibilizado pelo software TESTFACT Bock et al. (2003). Além disso, é importante dizer que o procedimento de restringir os valores dos parâmetros de discriminação à valores não-negativos pode até não eliminar por completo a invariância rotacional mas ao menos ameniza no sentido de que o conjunto de possíveis rotações que levam à invariância fica reduzido (veja, por exemplo, Jackman (2001) e Rivers (2003)).

Matos, G. S.

$\mathrm{IME} / \mathrm{USP}$ 


\subsubsection{Sobre a Amostra a Posteriori Simulada}

As estimativas dos modelos 2d2PPN e 2d2PPSn foram obtidas a partir de amostras da distribuição a posteriori de tamanho 1.000. Para obter a amostra do modelo 2d2PPN (e 2d2PPSn) foram realizados saltos de tamanho 100 após ter descartado 100.000 (e 200.000) valores iniciais de uma cadeia de comprimento 200.000 (e 300.000). Apesar da ocorrência de altas autocorrelações associadas à alguns parâmetros de discriminação do modelo 2 d2PPN, gráficos de trajetória e estatísticas de Geweke (1992) forneceram indícios de convergência da cadeia. A convergência da cadeia do modelo 2d2PPSn ocorreu de modo análogo, sendo que altas autocorrelações também foram observadas na cadeia marginal do parâmetro de assimetria, assim como nas cadeias de alguns parâmetros de dificuldade ${ }^{\Uparrow}$. Autocorrelações de aproximadamente $0.20 \mathrm{com}$ um lag de 500 e correspondente tamanho efetivo de amostra (ESS) igual a 97 foram algumas destas ocorrências. O tempo de processamento para a geração das cadeias foi de aproximadamente 1 h e 50 min. para o modelo $2 \mathrm{~d} 2 \mathrm{PPN}$ e $3 \mathrm{hs}$ e 50 min. para o modelo 2d2PPSn em um computador Core 2 Dual com 2 Gb de memória RAM.

Após algumas análises preliminares e a observação de uma taxa de aceitação igual a 26\%, o parâmetro "tuning", $\boldsymbol{c}_{\boldsymbol{\delta}}^{2}$, necessário à execução do Metropolis-Hastings no Passo 10 do algoritmo DAGS, ficou definido no valor igual a $\boldsymbol{c}_{\boldsymbol{\delta}}^{2}=(0.006,0.006)$.

Para iniciar as cadeias, foram considerados coeficientes estimados de regressões logísticas para os parâmetros de dificuldade (ver Sub-seção 4.2.4.1) e valores iguais a 1 para todos os parâmetros de discriminação. Para os traços latentes e parâmetro de assimetria, vetores bidimensionais nulos foram considerados.

\subsubsection{Análise sobre a Acurácia das Estimativas}

Na Figura 5.8 apresentamos alguns gráficos informativos sobre a acurácia das estimativas dos itens obtidas pelos modelos 2d2PPN e 2d2PPSn. No Gráfico (a), observamos que esses modelos estimam de maneira bastante similar e com grande acurácia as Funções de Resposta ao Item (FRI's) dos 30 itens analisados, pois as Raízes dos Erros Quadráticos Médios $(R E Q M)$ são inferiores à 0.05. A partir dos Gráficos (b), (c) e (d) também é possível observar que as estimativas dos itens obtidas pelos dois modelos são bastante similares. É possível observar, ainda, um bom desempenho do método DAGS de estimação com relação à acurácia pois os resíduos absolutos referentes aos parâmetros de dificuldade e discriminação assumem, em geral, valores inferiores a 0.3 .

Uma análise das diferenças absolutas entre as médias amostrais da distribuição a posteriori dos traços latentes individuais e verdadeiros valores, nos revela que de modo

【 Lembramos que rigorosamente falando, os parâmetros de dificuldade são interceptos (ver Sub-seção 5.3.1).

Matos, G. S.

IME/USP 

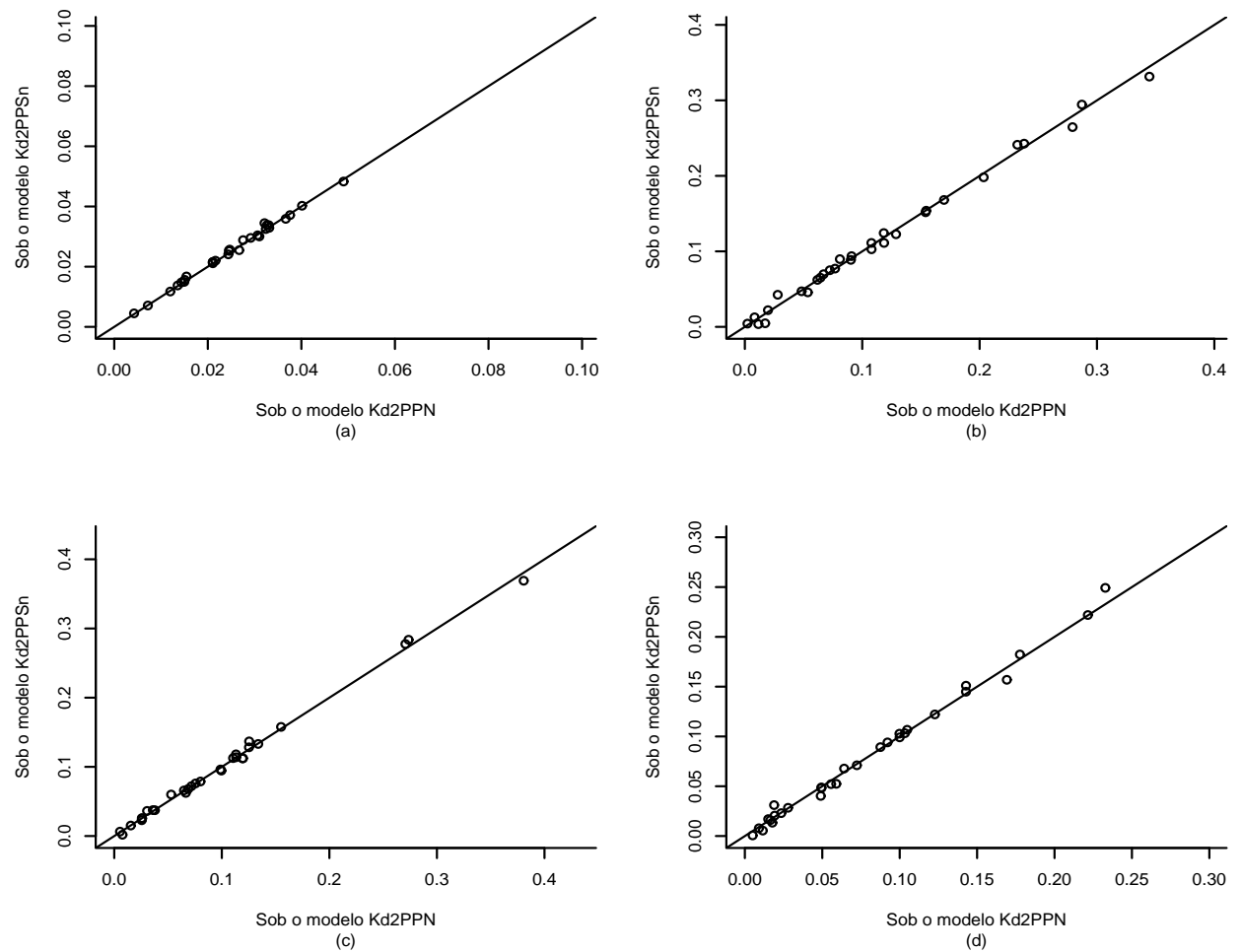

Figura 5.8 (a) Raizes dos Erros Quadráticos Médio dos itens $\left(R E Q M_{j}\right)$. Resíduos absolutos: (b) das dificuldades (c) das discriminações da dimensão 1 e (d) das discriminações da dimensão 2 .
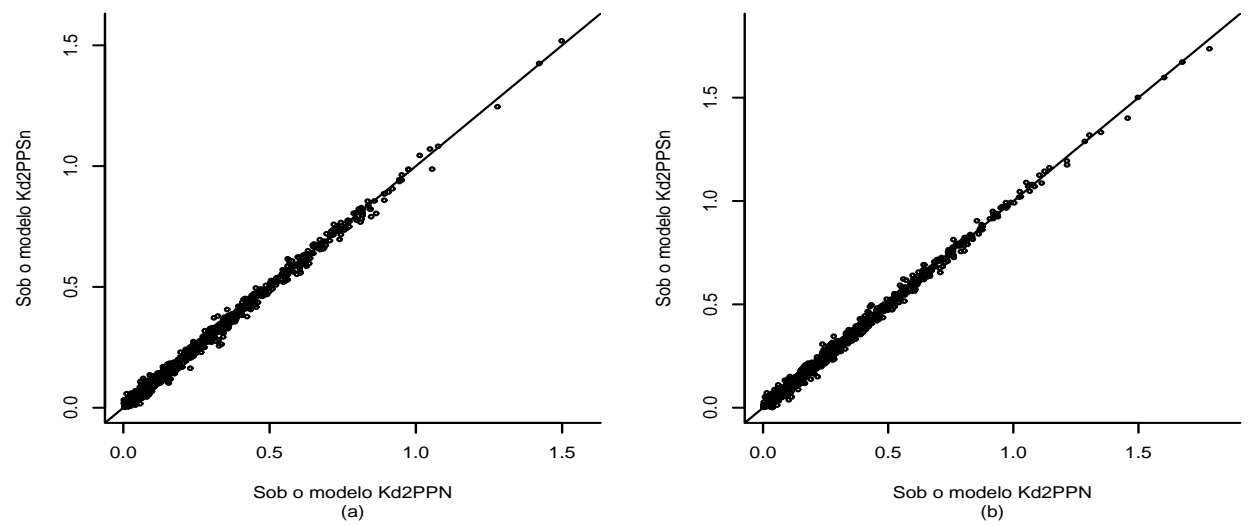

Figura 5.9 Resíduos absolutos: (a) dos traços latentes da dimensão 1 e (b) dos traços latentes da dimensão 2.

Matos, G. S. 
análogo às estimativas dos itens, as estimativas dos traços latentes obtidas pelos modelos 2d2PPN e 2d2PPSn são bastante similares. Este resultado pode ser observado a partir da Figura 5.9 a partir da qual também é possível observar a existência de alguns viéses com valores superiores a uma unidade. Com relação a estes grandes vieses, assim como foi no caso do teste unidimensional analisado na Seção 5.2.4, presumimos que independem do modelo adotado mas sim da capacidade de se construir testes com itens sob os mais variados níveis de dificuldade (e discriminação) de modo que as várias regiões da escala dos traços latentes sejam adequadamente representadas. A partir da Tabela 5.4, por exemplo, observamos que os níveis de dificuldade dos itens em cada teste variam entre -2.20 e 2.20. Enquanto isto, na Tabela 5.5, observamos que existem respondentes com valores dos traços latentes bem além destes limites.

\subsubsection{Análise das Estimativas Rotacionadas e Transladadas}

Conforme descrito na Seção 5.3.2, algumas medidas utilizadas em estudos sobre a capacidade de determinadas estimativas estimar os parâmetros de um modelo da TRI são dependentes da métrica. A Raíz do Erro Quadrático Médio e os resíduos absolutos são exemplos de tais medidas e foi por isso que os resultados descritos acima tomaram como base as estimativas transformadas para a métrica dos verdadeiros parâmetros.

Os resultados que apresentaremos em seguida não serão baseados em estimativas transformadas para a métrica dos verdadeiros parâmetros mas sim para uma métrica de mais fácil interpretação e possivelmente de maior coerência com as características do verdadeiro teste simulado (Seção 5.3.1). Tais transformações não trazem prejuízo algum quanto à análise de medidas, tais como: EAIC, EBIC, DIC e PsBF pois as mesmas são invariantes à métrica adotada em uma análise pois são todas baseadas em probabilidades e não em estimativas dos itens e traços latentes com métrica induzida por um determinado modelo. Neste contexto, o estudo desenvolvido na presente Sub-seção tem por objetivo ilustrar como é possível proceder a análise de um conjunto real de respostas aos itens através dos modelos multidimensionais da TRI, seja com distribuição a priori simétrica ou assimétrica para os vetores de traços latentes. evidenciada tal como foi observada nas Tabelas 5.11 e 5.12, ou seja, não foi evide É interessante lembrar que rotacionar as cargas fatoriais de uma solução particular de um modelo de análise fatorial (de variáveis contínuas ou discretas) é um procedimento comumente adotado na prática (Press, 2005; Bock; Aitkin, 1981).

A transformação utilizada na presente análise tomou como base o método de equalização desenvolvido por Reckase e Martineau (2004) e pode ser executada da seguinte forma:

1. A partir da matriz de discriminação estimada, $\widehat{\boldsymbol{\beta}}_{2}$, obter uma matriz de rotação

Matos, G. S.

IME/USP 
não-ortogonal $\boldsymbol{R}$ através, por exemplo, do método PROMAX. Em seguida, aplicar a seguinte transformação (rotação) à cada vetor de discriminação:

$$
\widetilde{\boldsymbol{\beta}}_{2 j}=\boldsymbol{R}^{\prime} \widehat{\boldsymbol{\beta}}_{2 j}, \quad j=1, \ldots, J
$$

2. Realizar uma translação do vetor de interceptos ("dificuldades") estimado $\widehat{\boldsymbol{\beta}}_{1}$, tomando como referência o vetor nulo $\boldsymbol{\beta}_{1 R e f}=\mathbf{0}$ :

$$
\widetilde{\beta}_{1 j}=\widehat{\beta}_{1 j}-\widehat{\boldsymbol{\beta}}_{2 j}^{\prime} \boldsymbol{R} \boldsymbol{b}, \quad j=1, \ldots, J,
$$

$\operatorname{com} \boldsymbol{b}=\left[\left(\widehat{\boldsymbol{\beta}}_{1}-\boldsymbol{\beta}_{1 R e f}\right)^{\prime} \widetilde{\boldsymbol{\beta}}_{2}\left(\widetilde{\boldsymbol{\beta}}_{2}^{\prime} \widetilde{\boldsymbol{\beta}}_{2}\right)^{-1}\right]^{\prime}$.

3. Realizar uma transformação de localização, escala e rotação sobre os traços latentes individuais estimados:

$$
\widetilde{\boldsymbol{\theta}}_{i}=\boldsymbol{R}^{-1} \widehat{\boldsymbol{\theta}}_{i}-\boldsymbol{b}, \quad i=1, \ldots, N
$$

Ao analisar os critérios de ajuste EAIC e EBIC constantes na Tabela 5.6, observamos que há uma leve evidência de que o modelo 2d2PPN se ajusta melhor aos dados. Esta ligeira vantagem do modelo $2 \mathrm{~d} 2 \mathrm{PPN}$ sobre o $2 \mathrm{~d} 2 \mathrm{PPSn}$ certamente é devida à maior penalização deste último por conter 2 parâmetros a mais associados à assimetria. Ao adotar o DIC na comparação dos modelos, estes resultados ficam ainda mais evidentes pois o DIC do modelo 2d2PPN também é ligeiramente menor que o DIC do modelo 2d2PPSn sendo que é a complexidade ou o número efetivo de parâmetros do modelo $2 \mathrm{~d} 2 \mathrm{PPSn}, p_{D}$, que essencialmente determina a diferença observada entre os DIC's (ver Tabela 5.7). A partir da Tabela 5.7 é possível observar que as médias dos desvios, $\bar{D}$, associadas aos dois modelos são praticamente iguais e é por isso que a complexidade do modelo foi essencial para determinar a pequena diferença entre os ajustes dos modelos. A partir desta tabela, também é possível observar que os erros padrão e.p $p_{N}$ e e.p $p_{T s}$ associados à função $\bar{D}$ dos dois modelos são relativamente pequenos e praticamente iguais. Estes últimos resultados, além da indicação sobre a comparabilidade das medidas DIC e $p_{D}$ entre os modelos, nos fornece uma boa indicação quanto a acurácia dos mesmos pois lembramos que tais medidas estão sujeitas à erros amostrais de Monte Carlo e que o cálculo preciso do erro-padrão para o DIC ainda é objeto de pesquisa (Spiegelhalter et al., 2002, pág. 607). Um Pseudo Fator de Bayes igual a 2.680 em uma escala 2log (duas vezes o logaritmo neperiano de PsBF), de acordo com Tabela calibrada por Raftery (1996) para um Fator de Bayes, também nos dá um indício moderadamente positivo a favor do modelo $2 \mathrm{~d} 2 \mathrm{PPN}$ quando comparado ao 2d2PPSn.

Quando avaliamos a capacidade dos modelos 2d2PPN e 2d2PPSn em recuperar a estrutura aproximadamente simples da verdadeira matriz de discriminações (Seção 5.3.1), 
Tabela 5.6 Esperanças da distribuição a posteriori estimadas de alguns critérios de informação.

\begin{tabular}{lcc}
\hline \hline \multirow{2}{*}{ Modelo } & \multicolumn{2}{c}{ Critério de Ajuste } \\
\cline { 2 - 3 } & EAIC & EBIC \\
\hline 2 d2PPN & 18832.37 & 32497.39 \\
$2 \mathrm{~d} 2 \mathrm{PPSn}$ & 18836.10 & 32517.28 \\
\hline \hline
\end{tabular}

Tabela 5.7 Critério de Informação do Desvio (DIC) e medidas relacionadas.

\begin{tabular}{lcccccc}
\hline \hline Modelo & $\bar{D}$ & e.p ${ }_{N}^{*}$ & e.p $p_{T s}^{* *}$ & $D(\overline{\boldsymbol{\theta}}, \overline{\boldsymbol{\beta}})$ & $p_{D}$ & \multirow{2}{*}{$D I C^{\dagger}$} \\
\hline 2d2PPN & 15452.37 & 1.862 & 1.932 & 14178.15 & 1274.216 & 16726.59 \\
2d2PPSn & 15452.10 & 1.963 & 2.302 & 14176.64 & 1275.461 & 16727.56 \\
\hline \hline
\end{tabular}

${ }^{*} e . p_{N}$ : erro-padrão Naive da medida $\bar{D} .{ }^{* *} e \cdot p_{T s}$ : erro-padrão Time-series da medida $\bar{D} . \dagger D I C=$ $D(\overline{\boldsymbol{\theta}}, \overline{\boldsymbol{\beta}})+2 p_{D}=\bar{D}+p_{D}$.

observamos a partir da Figura 5.10 que ambos modelos cumprem bem este papel. Através dos Gráficos (a) e (b) desta figura é possível observar que isto ocorreu de maneira bastante similar sendo que isto já era algo esperado tendo em vista que já haviamos observado uma forte relação linear entre os resíduos absolutos dos parâmetros de discriminação (Gráficos (c) e (d) da Figura 5.8). A partir do Gráfico (a) também é possível observar que as discriminações da dimensão 1 estimadas pelo modelo 2d2PPN acabaram sendo represen-

tadas pelos itens do sub-teste 2 (os últimos 15 itens da Tabela 5.4) enquanto que as discriminações da dimensão 2 acabaram sendo representadas pelos itens do sub-teste 1 (os 15 primeiros itens da Tabela 5.4). De acordo com nossa experiência e de alguns autores da literatura da TRI como Jackman (2001), isto ocorre devido a ausência de restrições mais significativas sobre a matriz de discriminação $\boldsymbol{\beta}_{2}$ pois, segundo descrição feita na Sub-seção 5.3.3.1, a única restrição feita sobre os elementos desta matriz foi a de nãonegatividade de seus valores, havendo ausência, portanto, de restrições que identificasse de modo expressivo qual item tem por objetivo avaliar as dimensões 1 e 2 do vetor de traços latentes. Note que tal comportamento não ocorreu com relação as discriminações estimadas pelo modelo 2d2PPSn (Gráfico (b) da Figura 5.10). Entretanto, é importante dizer que isto aconteceu por acaso, pois, assim como aconteceu em Bock e Aitkin (1981) e Jöreskog (1969, p. 195), tais discriminações estimadas correspondem a uma solução arbitrária para a qual o algoritmo computacional conseguiu convergir (veja também Subseção 3.3.1.2). Ressaltamos, ainda, que em outras simulações realizadas sob condições 


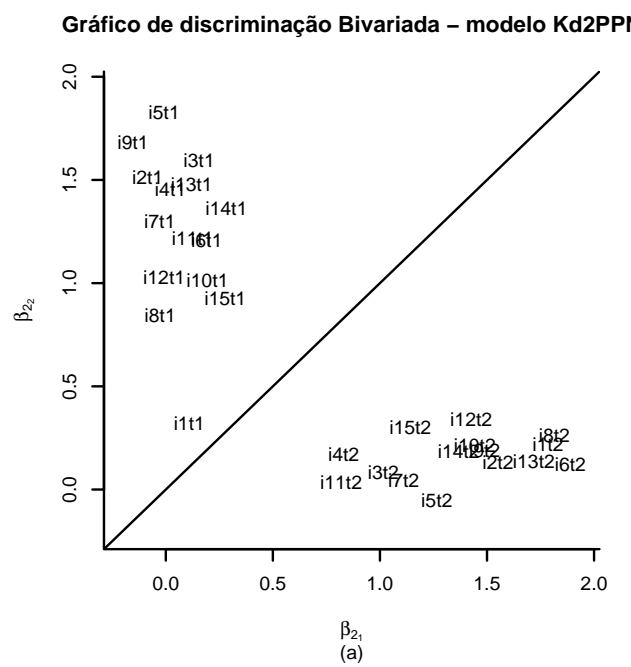

Gráfico de discriminação Bivariada - modelo Kd2PPSn

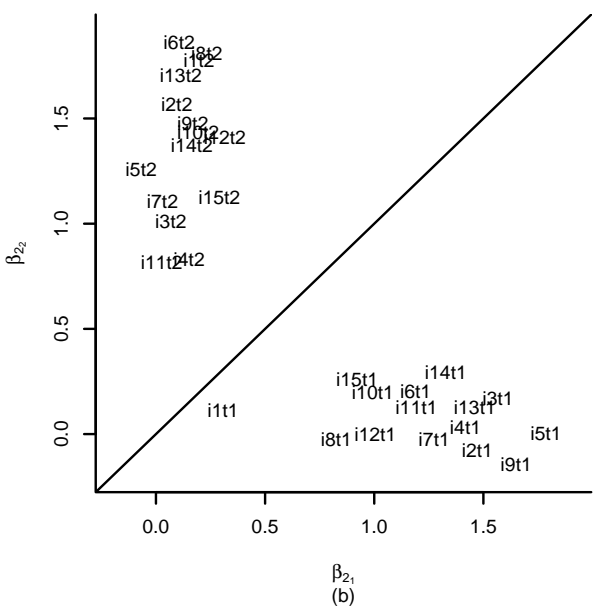

Figura 5.10 Estimativas (rotacionadas) das discriminações: (a) Sob o modelo 2d2PPN (b) Sob o modelo 2d2PPSn.

análogas, as dimensões representadas pelo modelo 2d2PPSn comportaram-se tal como as representadas aqui pelo modelo $2 \mathrm{~d} 2 \mathrm{PPN}$.

A análise de algumas medidas descritivas das estimativas médias dos traços latentes apresentadas na Tabela 5.8 nos revela que, de modo geral, tanto o modelo 2 d2PPN quanto o 2d2PPSn viabiliza interpretações relativamente coerentes com as características do verdadeiro teste simulado (Seção 5.3.1). Se, por exemplo, levarmos em consideração que os níveis médios de dificuldade dos subteste 1 (itens de 1 a 15) e 2 (itens de 16 a 30) foram estimados pelo modelo 2d2PPSn como sendo iguais à -0.01 e -0.03 , respectivamente, e que as discriminações revelaram uma estrutura de teste aproximadamente simples, é possível concluir que a habilidade (traço latente) média dos respondentes associada à primeira dimensão, -0.360, é inferior ao nível médio de dificuldade do subteste 1 enquanto que a habilidade (traço latente) média da segunda dimensão, 0.321, é superior ao nível médio de dificuldade do subteste 2. No que conscerne às variabilidades das estimativas médias dos traços latentes das dimensões 1 e 2, observamos que as mesmas sub-estimam as verdadeiras. Em especial, observamos que a variabilidade associada à segunda dimensão

Matos, G. S. 
Tabela 5.8 Descritivas das estimativas médias dos traços latentes obtidas pelos modelos 2d2PPN e 2d2PPSn.

\begin{tabular}{lccccccccc}
\hline \hline 2d2PPN & Mín. & Q1 & Md & Q3 & Máx. & $\bar{\theta}$ & $s^{2}$ & $a_{3}$ & $r_{12}$ \\
\hline$\theta_{1}$ & -1.970 & -0.365 & 0.333 & 0.964 & 2.563 & 0.317 & 0.859 & -0.091 & 0.836 \\
$\theta_{2}$ & -2.394 & -0.958 & -0.357 & 0.260 & 2.104 & -0.344 & 0.852 & 0.068 & - \\
\hline \hline \multirow{2}{*}{$2 \mathrm{~d} 2 \mathrm{PPSn}$} & Mín. & Q1 & Md & Q3 & Máx. & $\bar{\theta}$ & $s^{2}$ & $a_{3}$ & $r_{12}$ \\
\hline$\theta_{1}$ & -2.441 & -0.980 & -0.368 & 0.235 & 2.117 & -0.360 & 0.878 & 0.065 & 0.838 \\
$\theta_{2}$ & -2.009 & -0.368 & 0.332 & 0.979 & 2.614 & 0.321 & 0.888 & -0.091 & - \\
\hline
\end{tabular}

Notação: Q1 e Q3: 1ํㅡ e $3 \underline{\underline{0}}$ Quartis, Md: mediana, $\bar{\theta}$ : média amostral, $s^{2}$ : variância amostral, $a_{3}$ : assimetria amostral e $r_{12}$ : correlação amostral entre os traços latentes da dimensão 1 e 2 .

Matos, G. S. 
do vetor de traços latentes é a que mais sub-estima e isto deve-se, em parte, ao fato da referida dimensão estar associada à existência de assimetria na distribuição populacional (Seção 5.3.1).

Com relação ao modelo $2 \mathrm{~d} 2 \mathrm{PPN}$ é possível chegar à conclusões análogas às descritas para o modelo 2d2PPSn, desde que tomemos o devido cuidado de observar que as estimativas obtidas por este modelo correspondem às dimensões invertidas das verdadeiras dimensões do teste simulado, conforme descrito anteriormente. No que diz respeito à assimetria, observamos que nenhum dos modelos evidenciam o fato de que a verdadeira distribuição bidimensional de traços latentes possui assimetria negativa na segunda dimensão. Além disso, tanto o modelo 2d2PPN quanto 2d2PPSn estima a correlação entre as componentes do vetor de traços latentes como sendo igual a 0.83 , de modo que é possível concluir que existe uma relação linear relativamente forte entre os traços latentes da dimensão 1 e 2. Lembramos apenas que esta correlação é verdadeiramente moderada e igual à 0.56 (ver Seção 5.3.1).

Na Sub-seção 5.3.2 procuramos esclarecer que nesta primeira análise do teste bidimensional não teríamos como um dos objetivos a estimação dos parâmetros de localização e escala da distribuição dos traços latentes mas sim o de realizar uma análise do tipo exploratória sobre a estrutura da matriz de discriminação. Com isto em mente e havendo a necessidade de contornar possíveis dificuldades no processo de estimação devido à falta de identificabilidade dos modelos 2d2PPN e 2d2PPSn, a estimação dos parâmetros foi realizada considerando-se distribuições a priori padrões para os traços latentes, ou seja, com hiperparâmetro associado à localização fixado no vetor nulo e matriz de escala na matriz identidade. Neste contexto, lembramos que todas estimativas obtidas ao final do processo de estimação devem ser interpretadas com relação a métrica estabelecida por $(\boldsymbol{\mu}, \boldsymbol{\Sigma})=(\mathbf{0}, \boldsymbol{I})$. A métrica induzida por este procedimento, por exemplo, é uma provável explicação para o fato do parâmetro de assimetria do modelo $2 \mathrm{~d} 2 \mathrm{PPSn}, \boldsymbol{\delta}=\left(\delta_{1}, \delta_{2}\right)^{\prime}$, só ser estimado de maneira razoável na primeira dimensão. Pois, na segunda dimensão, conforme pode ser observado na Tabela 5.9, nem mesmo o intervalo de credibilidade de $97.5 \%$ contém o verdadeiro valor de assimetria - 1.5. A percepção que temos é de que

Tabela 5.9 Verdadeiro parâmetro de assimetria $\boldsymbol{\delta}=\left(\delta_{1}, \delta_{2}\right)^{\prime}$ e medidas resumo da amostra da distribuição a posterori.

\begin{tabular}{lcccccccc}
\hline \hline \multirow{2}{*}{ Assimetria } & \multicolumn{9}{c}{ Intervalos de quantis } & & & \\
\cline { 2 - 6 } & $2.5 \%$ & $25 \%$ & $50 \%$ & $75 \%$ & $97.5 \%$ & Média & D.P. & Verdadeiro \\
\hline$\delta_{1}$ & -0.63 & -0.29 & -0.11 & 0.07 & 0.54 & -0.10 & 0.29 & 0.0 \\
$\delta_{2}$ & -0.42 & -0.00 & 0.17 & 0.34 & 0.66 & 0.16 & 0.26 & -1.5 \\
\hline \hline
\end{tabular}

Matos, G. S.

$\mathrm{IME} / \mathrm{USP}$ 
as estimativas médias das assimetrias de aproximadamente -0.10 e 0.16 (ante-penúltima coluna da Tabela 5.9) tenderam a estimar não as verdadeiras assimetrias $\left(\delta_{1}=0\right.$ e $\delta_{2}=$ -1.5) mas sim os verdadeiros valores esperados da distribuição dos traços latentes, $E\left(\theta_{1}\right)=$ -0.50 e $E\left(\theta_{2}\right)=0.50$. Isto, de certa forma faz sentido, pois o valor esperado (e matriz de covariâncias) da distribuição assimétrica é função tanto do parâmetro de localização (e escala) quanto da assimetria (ver expressões em 5.1) e, como já mencionado, os parâmetros de localização $\mu_{\theta_{1}}$ e $\mu_{\theta_{2}}$ foram fixados no valor nulo. Para reforçar a plausibilidade de tais interpretações, informamos que o vetor de assimetria foi satisfatoriamente estimado em uma simulação (não apresentada aqui) em que os verdadeiros parâmetros de localização eram nulos. Para concluir podemos dizer que as interpretações referentes às estimativas do vetor de assimetria devem ser cautelosas na situação em que os parâmetros de localização e escala não são estimados juntamente com os demais parâmetros do modelo, em especial quando há suspeita de que os verdadeiros parâmetros de localização não são nulos.

Para finalizar esta etapa da análise (exploratória) do teste bidimensional simulado é possível concluir que tanto as estimativas obtidas pelo modelo $2 \mathrm{~d} 2 \mathrm{PPN}$ quanto $2 \mathrm{~d} 2 \mathrm{PPSn}$ via método DAGS, possibilitam a análise e interpretação de resultados coerentes com as verdadeiras e, em geral, desconhecidas características de um teste real. Além disso, é possível observar que não há diferenças significativas entre as estimativas dos itens e traços latentes obtidas pelos dois modelos. Conforme observado nesta seção, um aspecto desfavorável à adoção do modelo 2d2PPSn surge quando os parâmetros de localização e escala são fixados no vetor nulo e matrix identidade, respectivamente, pois os parâmetros do vetor de assimetria não foram adequadamente estimados sob esta condição e, de acordo com investigações adicionais, isto ocorre especialmente quando os verdadeiros parâmetros de localização não são nulos. Sob tais condições recomendamos cautela sobre a interpretação do vetor de assimetria estimado pois estudos adicionais sobre uma possível correção de viés sobre esta característica fazem-se necessários.

Uma importante contribuição da análise exploratória realizada nesta Seção revela que na prática é de suma importância a transformação de localização, escala e rotação das estimativas originalmente obtidas. Pois, foi somente assim que as interpretações tornaram-se mais simples e relativamente coerentes com as verdadeiras características do teste simulado. Neste contexto, nós apresentamos um método de transformação possivelmente novo no sentido de que o mesmo é uma adaptação do método de Reckase e Martineau (2004), utilizado na equalização de testes multidimensionais (Sub-seção 5.3.3.4).

Matos, G. S.

$\mathrm{IME} / \mathrm{USP}$ 


\subsubsection{Informações e Resultados sob os modelos 2m2PPN e 2m2PPSn}

\subsubsection{Sobre a Amostra a Posteriori Simulada}

As análises descritas nesta Seção baseam-se em amostras de tamanho 1.000 sendo que a amostra da distribuição a posteriori do modelo $2 \mathrm{~m} 2 \mathrm{PPN}$ foi obtida após descartar 80.000 valores iniciais e armazenar os valores de cada 100 itribuição terações de uma cadeia de comprimento 180.000 e, por motivos de convergência, a amostra da distribuição posteriori do modelo 2m2PPSn foi obtida após descartar 200.000 valores iniciais simulados e realizar saltos de tamanho 200 sobre uma sequência adicional de 200.000 valores. Para simular cada uma destas cadeias gastou-se em um computador Core 2 Dual (2Gb RAM) aproximadamente $1 \mathrm{~h}$ e $45 \mathrm{~min}$. e $6 \mathrm{hs}$ e $15 \mathrm{~min}$., respectivamente.

De maneira similar ao que observamos nas análises anteriores, os parâmetros de assimetria do modelo 2m2PPSn foram os que mais autocorrelação apresentaram (ver Gráficos (a1) e (b1) da Figura 5.11). Isto ocorreu especialmente com a assimetria associada à segunda dimensão da distribuição do vetor de traços latentes que conforme descrito na Seção 5.3.1 está relacionado a uma assimetria moderadamente negativa. Nos Gráficos (c1) e (d1) da Figura 5.11 é possível observar que os parâmetros de localização da distribuição dos traços latentes também apresentam altas autocorrelações, o que dificulta a obtenção de amostras representativas dos correspondentes espaços paramétricos. Apesar destas dificuldades, observamos nos correspondentes gráficos de trajetória alguns indícios de convergência destes parâmetros (coluna direita da Figura 5.11). Estatísticas de Geweke (Geweke, 1992) iguais a 0.41 e -3.1175 para os parâmetros de assimetria (dimensões 1 e 2) e -0.4507 e 2.9421 para os parâmetros de localização (dimensões 1 e 2) também nos permite aceitar com certa tolerância estes indícios de convergência. Um resultado que nos chama a atenção deve-se aos valores esperados marginais da distribuição dos vetores de traços latentes que sendo combinação linear dos parâmetros de localização e assimetria não apresentaram problema algum com relação à alta autocorrelação e convergência (ver Figura 5.12). Este é mais um resultado que nos faz aceitar o indício de convergência da cadeia do modelo $2 \mathrm{~m} 2 \mathrm{PPSn}$. Com relação ao modelo $2 \mathrm{~m} 2 \mathrm{PPN}$ nenhuma das cadeias marginais dos parâmetros evidenciaram problemas de altas autocorrelações. Análises gráficas de trajetória bem como estatísticas de Geweke também evidenciaram a convergência da cadeia.

Matos, G. S.

IME/USP 


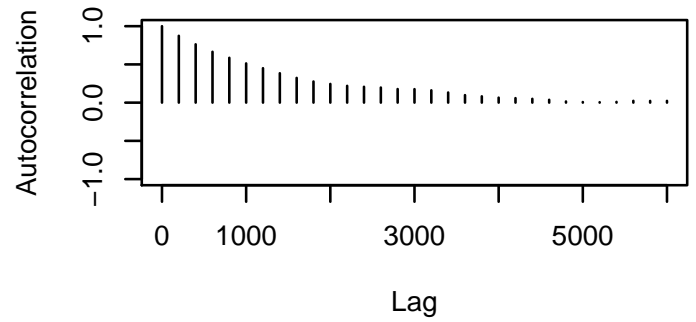

(a1) Assimetria dimensão 1

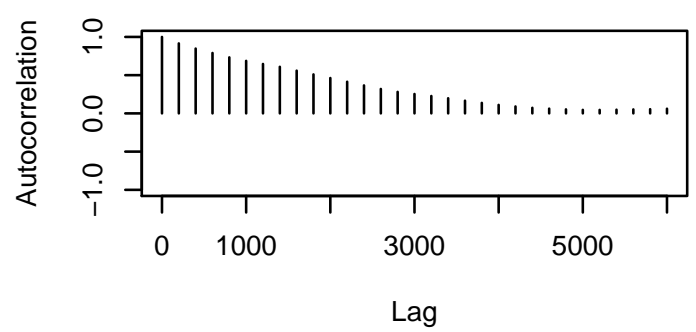

(b1) Assimetria dimensão 2

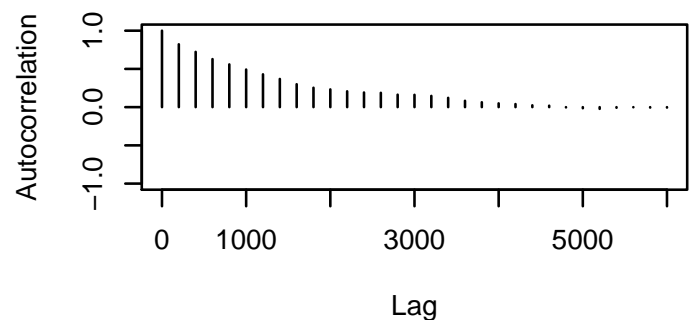

(c1) Locação dimensão 1

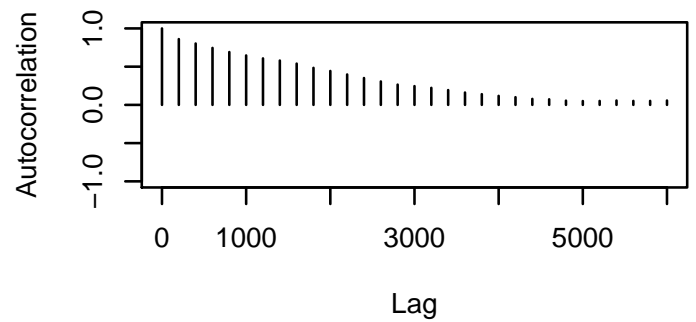

(d1) Locação dimensão 2

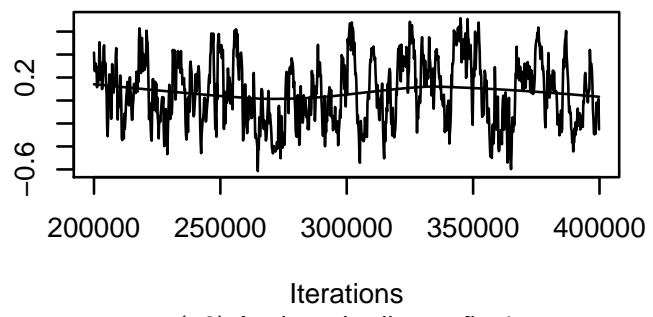

(a2) Assimetria dimensão 1

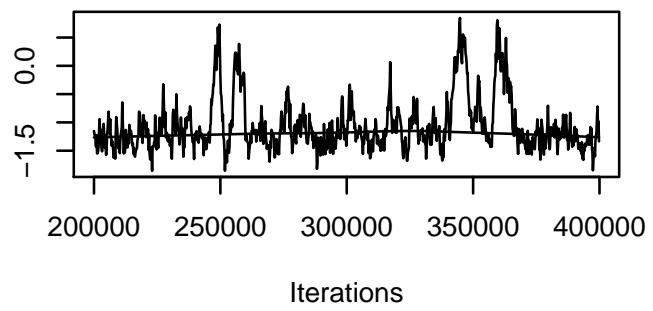

(b2) Assimetria dimensão 2

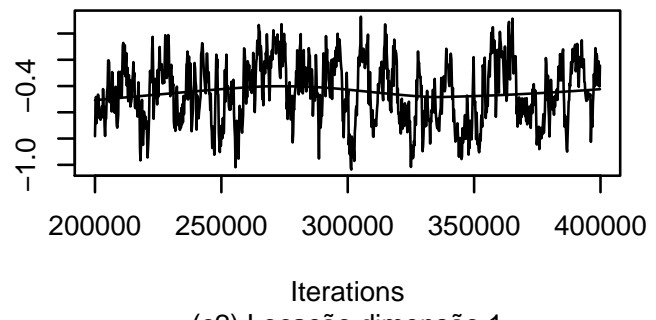

(c2) Locação dimensão 1

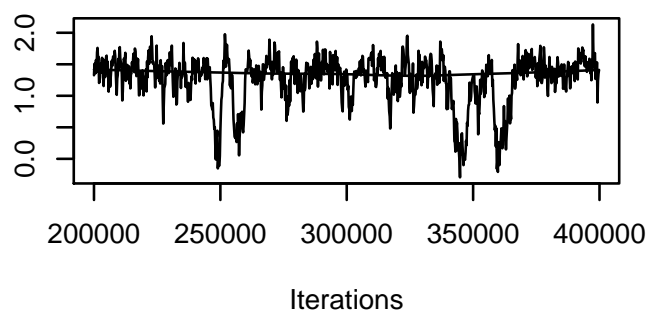

(d2) Locação dimensão 2

Figura 5.11 Autocorrelações e gráficos de trajetória dos parâmetros do modelo 2m2PPSn com maior dificuldade de convergência/autocorrelação.

Matos, G. S. 

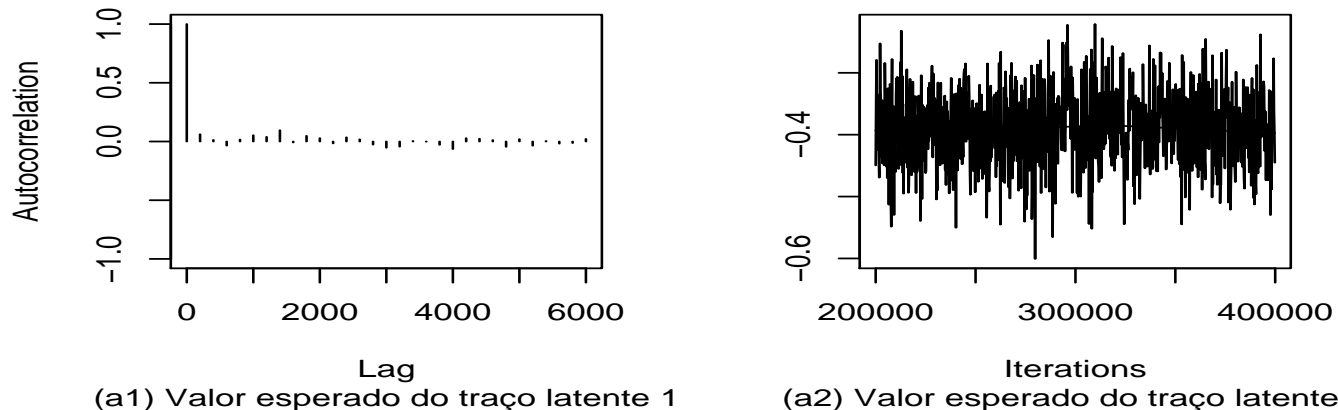

(a2) Valor esperado do traço latente 1
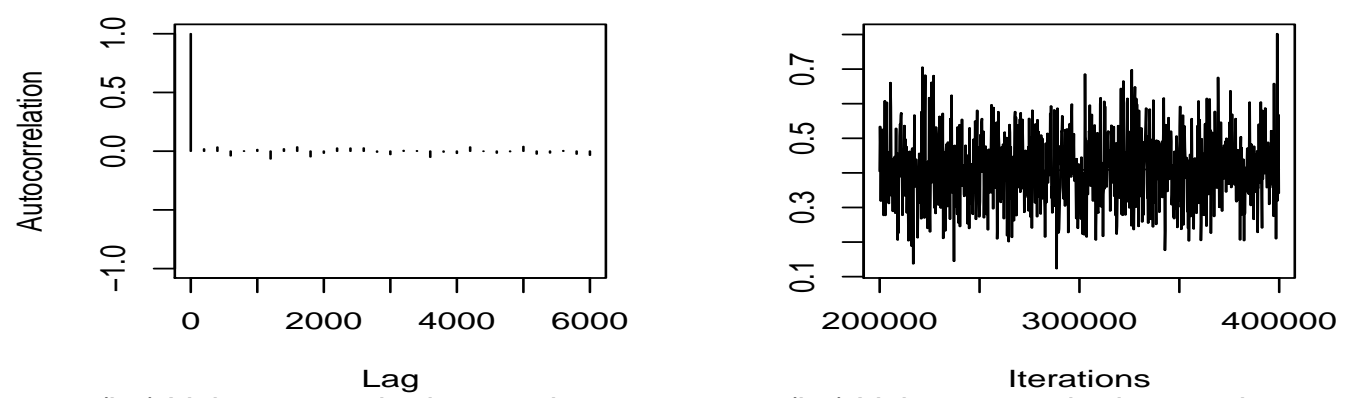

(b2) Valor esperado do traço latente 2

Figura 5.12 Autocorrelações e gráficos de trajetória dos valores esperados marginais da distribuição dos vetores dos traços latentes segundo o modelo 2m2PPSn.

\subsubsection{Sobre as Distribuições a Priori e os Valores Iniciais para o Algoritmo DAGS}

Para as análises desenvolvidas na presente sub-seção é importante saber que as distribuições a priori e os valores iniciais para os parâmetros dos itens (livres) e traços latentes foram especificadas de maneira análoga ao descrito na Sub-seção 5.3.3. Com relação aos parâmetros populacionais, distribuições pouco informativas foram adotadas. Para os parâmetros de localização e assimetria, por exemplo, prioris normais independentes com média zero e variância 100 foram especificadas e, para a matriz de precisão $\boldsymbol{Q}=\boldsymbol{\Sigma}_{\boldsymbol{\theta}}^{-1}$ uma distribuição Wishart $_{2}\left(2, \boldsymbol{I}_{2}\right)$. O parâmetro "tuning" $\boldsymbol{c}_{\boldsymbol{\delta}}^{2}=(0.0015,0.0015)$ necessário à amostragem do vetor de parâmetros de assimetria do modelo $2 \mathrm{~m} 2 \mathrm{PPSn}$ forneceu uma taxa de aceitação de $26,7 \%$ e na inicialização dos parâmetros de localização e precisão, $\boldsymbol{\mu}_{\theta}$ e $\boldsymbol{Q}$, o vetor nulo e a matriz identidade foram adotados.

Matos, G. S.

IME/USP 


\subsubsection{Análise Comparativa entre os Modelos 2m2PPN e 2m2PPSn}

As análises comparativas entre os modelos 2m2PPN e 2m2PPSn, a exemplo das anteriores, não nos revela diferença significativa entre a acurácia das estimativas obtidas por estes modelos. Observando-se, por exemplo, as Figuras 5.13 e 5.14 podemos verificar que as Raízes dos Erros Quadráticos Médio dos itens bem como os erros absolutos dos parâmetros dos itens e traços latentes são bastante similares entre os modelos investigados.

A partir da Tabela 5.10 observamos que enquanto as medidas EAIC e EBIC com pouca intensidade favorecem o modelo $2 \mathrm{~m} 2 \mathrm{PPN}$, o DIC de forma pouco expressiva favorece o modelo 2m2PPSn. Esta contradição pode ser atribuída às diferentes formas que estes critérios penalizam os modelos com relação à quantidade de parâmetros (Brooks, 2002) de modo que, em termos práticos, é possível concluir que não há diferença significativa entre os ajustes aos dados segundo os modelos $2 \mathrm{~m} 2 \mathrm{PPN}$ e $2 \mathrm{~m} 2 \mathrm{PPSn}$. O valor do PsBF na escala 2log igual a 1.042 a favor do modelo 2m2PPSn e contra o 2m2PPN; segundo Tabela 10.1 de calibração de Raftery (1996); também aponta para esta conclusão.

Nas Tabelas 5.11 e 5.12 encontram-se os verdadeiros parâmetros populacionais dos traços latentes e as estimativas obtidas pelos modelos 2m2PPN e 2m2PPSn, respectivamente. Como pode ser observado tanto os valores esperados quanto as variâncias e covariâncias/correlações populacionais foram estimados(das) de maneira bastante semelhante pelos dois modelos. É possível observar também que ambos modelos estimaram os valores esperados da distribuição dos traços latentes de maneira eficiente, existindo apenas um viés aproximado de 0.1 quando consideradas as médias da amostra da distribuição a posteriori. Quando os intervalos quantílicos relacionados aos dois componentes do vetor de valores esperados são considerados, é possível observar que não há intersecção nem mesmo entre os intervalos de $95 \%$ de credibilidade; o que evidencia de modo correto a existência de uma diferença significativa entre os valores esperados associados à primeira e segunda dimensão da distribuição do vetor de traços latentes. Com relação às variâncias da distribuição dos vetores dos traços latentes a estimação bem sucedida não se repetiu. Pois, enquanto a variância correspondente a primeira dimensão foi sub-estimada; a variância associada à segunda dimensão foi consideravelmente super-estimada (observar verdadeiras variâncias e estimativas médias nas Tabelas 5.11 e 5.12). Apesar disto, uma relação de ordem foi mantida, ou seja, a variância associada à distribuição da primeira dimensão do vetor dos traços latentes foi estimada como sendo menor do que a associada à segunda dimensão. Em termos de correlação, observa-se uma super-estimação de magnitude 0.25 o que nos faz inferir sobre uma correlação positiva e relativamente forte entre os traços latentes quando verdadeiramente é moderada. Com relação aos parâmetros de assimetria estimados pelo modelo $2 \mathrm{~m} 2 \mathrm{PPSn}$, os mesmos foram estimados de maneira relativamente satisfatória tendo em vista que é possível inferir sobre uma assimetria nula e negativa para a primeira e segunda dimensão da distribuição dos vetores dos traços latentes, respectivamente (ver as duas últimas linhas da Tabela 5.12).

Matos, G. S.

IME/USP 

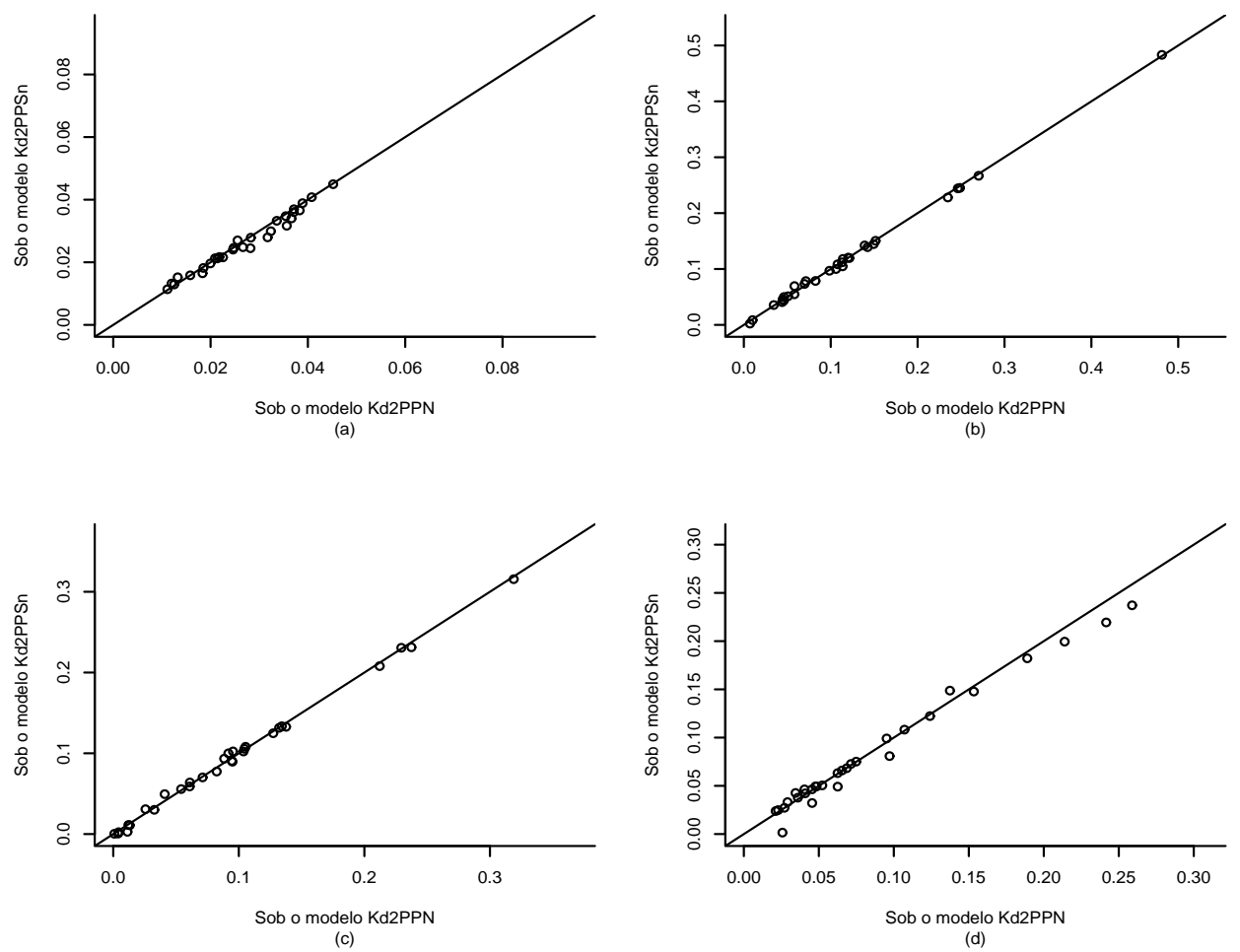

Figura 5.13 (a) Raizes dos Erros Quadráticos Médio dos itens $\left(R E Q M_{j}\right)$. Resíduos absolutos: (b) das dificuldades (c) das discriminações da dimensão 1 e (d) das discriminações da dimensão 2. *Nos gráficos acima, onde houver d leia-se $m$.
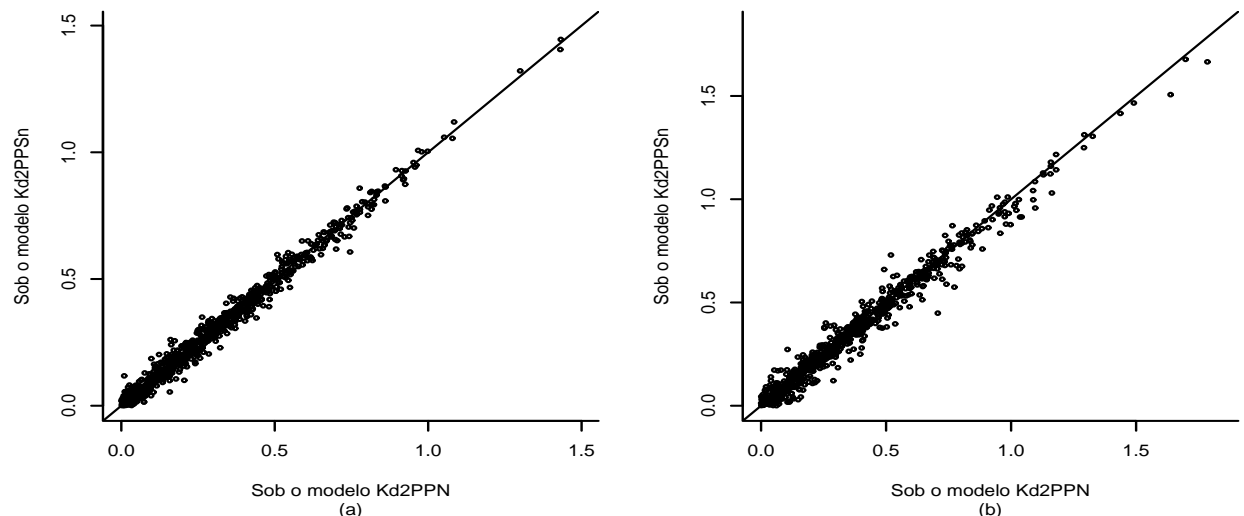

Figura 5.14 Resíduos absolutos: (a) dos traços latentes da dimensão 1 e (b) dos traços latentes da dimensão 2. *Nos gráficos acima, onde houver d leia-se m.

Matos, G. S. 
Tabela 5.10 Medidas de critério de ajuste e seleção de modelos.

\begin{tabular}{lcccc}
\hline \hline \multirow{2}{*}{ Modelo } & \multicolumn{2}{c}{ Critério de Ajuste } & \multirow{2}{*}{ Critério de Seleção } \\
\cline { 2 - 5 } & DIC $\left(p_{D}\right)$ & EAIC & EBIC & $2 \log (P s B F)$ \\
\hline \multirow{2}{*}{$2 \mathrm{~m} 2 \mathrm{PPN}$} & $16756.33(1281.607)$ & 18796.73 & 32227.26 & -1.042 \\
$2 \mathrm{~m} 2 \mathrm{PPSn}$ & $16750.91(1278.284)$ & 18798.62 & 32245.33 & 1.042 \\
\hline \hline
\end{tabular}

Tabela 5.11 Parâmetros populacionais e estimativas da amostra da distribuição a posteriori obtidas sob o modelo 2 m2PPN.

\begin{tabular}{|c|c|c|c|c|c|c|c|c|}
\hline \multirow{2}{*}{ Parâmetro } & \multirow[b]{2}{*}{ Verdadeiro } & \multirow[b]{2}{*}{ Média } & \multirow[b]{2}{*}{ D.P. } & \multicolumn{5}{|c|}{ Intervalos de quantis } \\
\hline & & & & $2.5 \%$ & $25 \%$ & $50 \%$ & $75 \%$ & $97.5 \%$ \\
\hline$E\left(\theta_{1}\right)$ & -0.50 & -0.39 & 0.06 & -0.51 & -0.43 & -0.39 & -0.35 & -0.27 \\
\hline$E\left(\theta_{2}\right)$ & 0.50 & 0.42 & 0.10 & 0.25 & 0.35 & 0.41 & 0.48 & 0.61 \\
\hline $\operatorname{Var}\left(\theta_{1}\right)$ & 0.90 & 0.75 & 0.10 & 0.57 & 0.68 & 0.74 & 0.81 & 0.97 \\
\hline $\operatorname{Var}\left(\theta_{2}\right)$ & 1.52 & 3.21 & 0.54 & 2.31 & 2.83 & 3.15 & 3.51 & 4.44 \\
\hline $\operatorname{Cor}\left(\theta_{1}, \theta_{2}\right)$ & 0.56 & 0.81 & 0.02 & 0.78 & 0.80 & 0.81 & 0.82 & 0.84 \\
\hline
\end{tabular}

Tabela 5.12 Parâmetros populacionais e estimativas da distribuição a posteriori sob o modelo 2m2PPSn.

\begin{tabular}{lllllllll}
\hline \hline \multirow{2}{*}{ Parâmetro } & & & & \multicolumn{5}{c}{ Intervalos de quantis } \\
\cline { 5 - 9 } & Verdadeiro & Média & D.P. & $2.5 \%$ & $25 \%$ & $50 \%$ & $75 \%$ & $97.5 \%$ \\
\hline$E\left(\theta_{1}\right)$ & -0.50 & -0.39 & 0.06 & -0.50 & -0.43 & -0.39 & -0.35 & -0.27 \\
$E\left(\theta_{2}\right)$ & 0.50 & 0.41 & 0.10 & 0.23 & 0.35 & 0.41 & 0.47 & 0.61 \\
\hline $\operatorname{Var}\left(\theta_{1}\right)$ & 0.90 & 0.76 & 0.10 & 0.59 & 0.69 & 0.75 & 0.83 & 0.99 \\
$\operatorname{Var}\left(\theta_{2}\right)$ & 1.52 & 3.32 & 0.53 & 2.42 & 2.94 & 3.26 & 3.64 & 4.51 \\
$\operatorname{Cor}\left(\theta_{1}, \theta_{2}\right)$ & 0.56 & 0.81 & 0.02 & 0.77 & 0.80 & 0.81 & 0.82 & 0.84 \\
\hline$\delta_{1}$ & 0.00 & 0.07 & 0.28 & -0.42 & -0.15 & 0.07 & 0.28 & 0.59 \\
$\delta_{2}$ & -1.5 & -1.06 & 0.47 & -1.62 & -1.35 & -1.18 & -0.95 & 0.29 \\
\hline \hline
\end{tabular}

Matos, G. S. 


\subsubsection{Sobre a Identificabilidade dos Modelos 2m2PPN e 2m2PPSn}

Uma informação de grande importância para que os verdadeiros valores esperados da distribuição dos traços latentes fossem satisfatoriamente estimados deve-se à forma como introduzimos no processo de estimação a identificabilidade dos modelos 2m2PPN e 2m2PPSn. Pois, para que as estimativas dos valores esperados dos traços latentes associados às dimensões 1 e 2 fossem estimados próximos de -0.5 e 0.5, respectivamente, foi essencial que fixássemos os parâmetros de dificuldade e discriminação de dois itens, um em cada subteste, tal como descrito por Béguin e Glas (2001). Estes autores com o intuito de estimar os parâmetros populacionais $\boldsymbol{\mu}$ e $\boldsymbol{\Sigma}_{\theta}$ propuseram que $K$ parâmetros de dificuldade ficassem fixados no valor zero além de considerar $\boldsymbol{\beta}_{2 j k}=1$ para $j, k=1, \ldots, K$ se $j=k$ e $\boldsymbol{\beta}_{2 j k}=0$ para $j, k=1, \ldots, K$ no caso em que $j \neq k \|$. Introduzir tais restrições no processo de estimação é uma tarefa relativamente simples, porém não é claro nesta abordagem quais itens de cada sub-teste devem ser fixados. A experiência adquirida por nós na análise do presente conjunto de dados nos revelou que também é essencial a escolha dos itens a serem fixados em cada um dos sub-testes. Pois a satisfatória recuperação dos verdadeiros valores esperados dos traços latentes ocorreu somente quando um item de cada teste com nível mediano de dificuldade (com relação ao percentual de acerto) foi fixado. Além disso, foi de suma importância que as restrições dos dois itens fossem estabelecidas nas duas primeiras linhas da matriz de discriminação, $\boldsymbol{\beta}_{2}$. Importante também foi a ordenação das colunas da base de dados de maneira que as duas primeiras colunas de respostas correspondessem aos dois itens fixados. Tal ordenação demonstrou-se importante para que uma correspondência direta entre as duas primeiras colunas da base de dados e as duas primeiras linhas da matriz $\boldsymbol{\beta}_{2}$ (discriminações dos correspondentes itens fixados) ficasse estabelecida. Fez-se necessário, ainda, a garantia de correspondência (ordenação) entre as demais colunas de respostas aos itens da base de dados e demais linhas da matriz de discriminação $\boldsymbol{\beta}_{2}$ de modo que a $j$-ésima coluna da matriz de dados e a $j$-ésima linha da matriz de discriminação estivesse diretamente associada ao $j$-ésimo item.

Apenas para descrever um pouco mais sobre nossa experiência com relação à identificabilidade dos modelos $2 \mathrm{~m} 2 \mathrm{PPN}$ e 2m2PPSn na situação em que os parâmetros de localização e escala da distribuição dos traços latentes também são estimados, observamos que ao fixar os itens mais fáceis (maiores percentuais de acerto aos itens) de cada teste, os valores estimados para as esperanças dos traços latentes ficaram em torno do valor 2. Por outro lado, quando os itens mais difícies (menores percentuais de acerto aos itens) de cada teste foram fixados; os valores estimados para as esperanças dos traços latentes ficaram em torno do valor -2. O fato agravante nestes casos foi que a diferença entre as esperanças estimadas nas duas dimensões não foi evidenciada tal como foi observada nas Tabelas 5.11 e 5.12, ou seja, não foi evidenciado que o valor esperado associado à primeira dimensão

|| Lembramos que sob os modelos analisados neste momento a matriz de discriminação, $\boldsymbol{\beta}_{2}$, já possui alguns elementos iguais a zero devido a estrutura de um modelo multivariado (Sub-Seção 3.2.2).

Matos, G. S.

$\mathrm{IME} / \mathrm{USP}$ 
da distribuição do vetor de traços latentes é aproximadamente uma unidade inferior ao da segunda dimensão. Tais resultados ilustram, portanto, que a estimação "bem" sucedida dos parâmetros populacionais associados à distribuição do vetor de traços latentes, além de depender de vários outros fatores (e.g. características e quantidades de itens; Andrade (2001)), também depende da forma como se elimina a identificabilidade do modelo de resposta ao item, seja no caso em que a distribuição dos traços latentes é normal multivariada simétrica ou assimétrica.

Apesar dos resultados acima descritos não terem sido observados sob várias outros possíveis conjuntos de dados, sugerimos que em uma análise de dados reais em que a estimação dos parâmetros de localização da distribuição dos traços latentes seja de interesse, os parâmetros dos itens medianamente difíceis sejam os escolhidos para serem fixados na abordagem de identificabilidade de Béguin e Glas (2001). Pois, de acordo com nossa experiência sobre as análises e interpretações dos modelos unidimensionais e bidimensionais apresentadas nas Sub-seções 5.2 e 5.3.3, respectivamente, é de suma importância que se tenha uma idéia sobre a relação de ordem e magnitude entre a(s) dificuldade(s) média(s) do(s) teste(s) e a(s) correspondente(s) média(s) do(s) traço(s) latente(s). Devemos observar, também, que nossa experiência evidencia que não somente a forma da distribuição dos traços latentes, se simétrica ou assimétrica, mas também o método e a forma como se resolve a falta de identificabilidade de um modelo da TRI, são fatores que determinam uma particular métrica que pode ou não ser de fácil interpretação. Neste caso o bom senso e a experiência sobre as circunstâncias em que o conjunto de dados de interesse é obtido tornam-se imprescindíveis para que o "melhor" resultado seja adotado e divulgado.

\subsubsection{Seleção de um Modelo e Análise Final}

Na Sub-seção 5.3.3 não foram identificadas diferenças significativas entre as estimativas e os ajustes dos modelos $2 \mathrm{~d} 2 \mathrm{PPN}$ e $2 \mathrm{~d} 2 \mathrm{PPSn}$ mas apenas uma leve evidência a favor do modelo 2d2PPN. Resultados similares foram obtidos quando comparados os modelos 2m2PPN e 2m2PPSn em que além dos parâmetros dos itens e traços latentes, os parâmetros de localização e escala da distribuição dos traços latentes também foram estimados (Sub-seção 5.3.4). Neste último caso, porém, a leve evidência de melhor ajuste aos dados favoreceu a escolha do modelo $2 \mathrm{~m} 2 \mathrm{PPSn}$ desde que as medidas DIC e PsBF fossem adotadas como critérios de seleção de modelos. Nas Tabelas 5.13, 5.14 e 5.15 novamente apresentamos algumas medidas de critério de ajuste e seleção de modelos associadas aos modelos 2d2PPN, 2d2PPSn, 2m2PPN e 2m2PPSn mas desta vez apresentamos também as medidas correspondentes a mais dois modelos que achamos conveniente considerar como candidatos a um melhor ajuste aos dados analisados nesta Seção.

Os dois modelos adicionais que denotamos por 2d2PPN(PP) e 2d2PPSn(PP) (PP: Parâmetros Populacionais) corresponde a uma combinação dos modelos antes analisados.

Matos, G. S.

$\mathrm{IME} / \mathrm{USP}$ 
Especificamente, os modelos 2d2PPN(PP) e 2d2PPSn(PP) caracterizam-se por uma estrutura bastante semelhante a dos modelos 2d2PPN e 2d2PPSn da Sub-seção 5.3.3 sendo que os parâmetros de discriminação dos itens i8t1 e i8t2 dos sub-testes 1 e 2 foram fixados conforme procedimento de identificabilidade descrito e discutido na Sub-seção 5.3.4.4. Por outro lado, os modelos $2 \mathrm{~d} 2 \mathrm{PPN}(\mathrm{PP})$ e $2 \mathrm{~d} 2 \mathrm{PPSn}(\mathrm{PP})$ também assemelham-se aos $2 \mathrm{~m} 2 \mathrm{PPN}$ e 2m2PPSn da Sub-seção 5.3.4 somente pelo fato dos hiperparâmetros de localização e escala da distribuição dos traços latentes também serem estimandos.

A partir das Tabelas 5.13, 5.14 e 5.15 diferentes decisões podem ser tomadas pois a escolha de um modelo deve levar em consideração não apenas o critério de melhor ajuste aos dados mas também é necessário levar em consideração as questões e os objetivos que se pretende responder/alcançar pela análise dos dados. Por exemplo, se o principal objetivo da análise do teste bidimensional é apenas a estimação e interpretação dos parâmetros dos itens e possivelmente dos traços latentes de cada um dos respondentes, parece-nos razoável adotar o modelo $\mathbf{2 d} \mathbf{2 P P N}$, tendo em vista que o DIC correspondente (Tabela 5.14) é o menor entre todos os demais. Além disso, como pode ser percebido na primeira linha da Tabela 5.15, o valor do PsBF só é desfavorável a este modelo quando comparado aos multivariados 2m2PPN e 2m2PPSn. Esta mesma conclusão é obtida quando considerados os critérios EAIC e EBIC constantes na Tabela 5.13. De outra forma, quando os objetivos da análise dos dados se extende à necessidade de se estimar e interpretar os parâmetros populacionais associados à distribuição dos vetores dos traços latentes, a decisão pela escolha do modelo 2m2PPSn pode ser considerada se adotamos o DIC e o PsBF como critérios de ajuste e seleção de modelos. É interessante notar que tal decisão não encontra suporte quando os critérios EAIC e EBIC são considerados e isto até certo ponto deve-se à maneira como penalizamos os modelos pois tal penalização depende da forma como consideramos o número de observações e parâmetros em um modelo complexo hierárquico; assim como são os modelos multidimensionais/multivariados aqui considerados. A determinação do número de observações e parâmetros para estes modelos é algo discutível sendo que o DIC ameniza este problema ao permitir que o analista indiretamente determine tais quantidades, através do que Spiegelhalter et al. (2002) chamam de foco da análise (verossimilhança ou "complexidade do modelo"; Brooks (2002) e Bazán (2005)). No presente trabalho, a exemplo de Bazán (2005), o foco da análise adotado, ou melhor, a verossimilhança considerada no cálculo do DIC foi a condicional expressa por (3.2) e devido a concordância entre os resultados evidenciados pelo DIC e PsBF, nós continuamos favoráveis à adoção do modelo 2m2PPSn quando além dos parâmetros dos itens e traços latentes, os populacionais são de interesse.

Matos, G. S.

$\mathrm{IME} / \mathrm{USP}$ 
Tabela 5.13 Esperanças da distribuição a posteriori estimadas de alguns critérios de informação.

\begin{tabular}{lcccccc}
\hline \hline \multirow{2}{*}{$\begin{array}{l}\text { Critério } \\
\text { de }\end{array}$} & \multicolumn{6}{c}{ Modelo } \\
\cline { 2 - 7 } Ajuste & $2 \mathrm{~d} 2 \mathrm{PPN}$ & $2 \mathrm{~d} 2 \mathrm{PPSn}$ & $2 \mathrm{~m} 2 \mathrm{PPN}$ & 2m2PPSn & 2d2PPN(PP) & 2d2PPSn(PP) \\
\hline EAIC & 18832.37 & 18836.10 & 18796.73 & 18798.62 & 18880.07 & 18878.63 \\
EBIC & 32497.39 & 32517.28 & 32227.26 & 32245.32 & 32537.00 & 32551.73 \\
\hline \hline
\end{tabular}

Finalmente, na situação em que não haja informações a priori que permitam admitir uma estrutura bivariada para o teste (conforme caracterizado na Sub-seção 3.2.2) e que além disso os parâmetros populacionais também são de interesse analisar, o conjunto de modelos a serem considerados se reduz ao $2 \mathrm{~d} 2 \mathrm{PPSn}(\mathrm{PP})$ e $2 \mathrm{~d} 2 \mathrm{PPN}(\mathrm{PP})$. Neste caso, tanto o DIC quanto o PsBF fornecem indícios pela escolha do modelo 2d2PPSn(PP) como o que melhor explica o comportamento dos dados (Tabelas 5.14 e 5.15).

Tabela 5.14 Critério de Informação do Desvio (DIC) e medidas relacionadas.

\begin{tabular}{lcccccl}
\hline \hline Modelo & $\bar{D}$ & e.p $p_{N}$ & e.p $p_{T s}$ & $D(\overline{\boldsymbol{\theta}}, \overline{\boldsymbol{\beta}})$ & $p_{D}$ & DIC \\
\hline 2d2PPN & 15452.37 & 1.862 & 1.932 & 14178.15 & 1274.216 & 16726.59 \\
2d2PPSn & 15452.10 & 1.963 & 2.302 & 14176.64 & 1275.461 & 16727.56 \\
\hline 2m2PPN & 15474.73 & 1.806 & 1.684 & 14193.12 & 1281.607 & 16756.33 \\
2m2PPSn & 15472.62 & 1.909 & 1.780 & 14194.34 & 1278.284 & 16750.91 \\
\hline 2d2PPN(PP) & 15502.07 & 1.974 & 2.609 & 14216.81 & 1285.255 & 16787.32 \\
2d2PPSn(PP) 15496.63 & 2.211 & 2.368 & 14217.58 & 1279.049 & 16775.68 \\
\hline \hline
\end{tabular}

Matos, G. S. 
Tabela 5.15 PsBF (na escala 2log)* associados à alguns pares de modelos.

\begin{tabular}{lcccccc}
\hline \multirow{2}{*}{ Modelos } & \multicolumn{7}{c}{ Modelos } \\
\cline { 2 - 7 } & 2d2PPN & 2d2PPSn & 2m2PPN & 2m2PPSn & 2d2PPN(PP) & 2d2PPSn(PP) \\
\hline $2 \mathrm{~d} 2 \mathrm{PPN}$ & - & 2.680 & -43.812 & -44.858 & 17.954 & 5.178 \\
$2 \mathrm{~d} 2 \mathrm{PPSn}$ & - & - & -46.493 & -47.538 & 15.273 & 2.498 \\
\hline $2 \mathrm{~m} 2 \mathrm{PPN}$ & - & - & - & -1.042 & 61.766 & 48.990 \\
$2 \mathrm{~m} 2 \mathrm{PPSn}$ & - & - & - & - & 62.811 & 50.036 \\
\hline $2 \mathrm{~d} 2 \mathrm{PPN}(\mathrm{PP})$ & - & - & - & - & - & -12.777 \\
$2 \mathrm{~d} 2 \mathrm{PPSn}(\mathrm{PP})$ & - & - & - & - & - & - \\
\hline \hline
\end{tabular}

*Segundo tabela de calibração de Raftery (1996) valores menores que 0 fornecem indícios contra o modelo aqui especificado na linha. Valores entre 0 e 2 indicam indiferença entre o ajuste dos modelos. Entre 2 e 5 evidência positiva ao modelo aqui especificado na linha. Entre 5 e 10 evidência forte e acima de 10 a evidência é muito forte de que o modelo, aqui especificado na linha, é o que melhor explica o comportamento dos dados. Note que ao multiplicar por -1 os valores indicados nesta tabela, interpretação análoga é obtida sendo que neste caso as conclusões devem se referir a favor (ou contra) o modelo especificado na coluna.

Matos, G. S. 
Na Tabela 5.16 encontram-se os verdadeiros parâmetros populacionais e as estimativas obtidas pelo modelo $2 \mathrm{~d} 2 \mathrm{PPSn}(\mathrm{PP})$. Uma breve comparação entre estas estimativas e aquelas obtidas pelo modelo 2d2PPSn (ver Tabela5.12) mostram que tanto a correlação entre os traços latentes quanto a assimetria associada à segunda dimensão da distribuição dos vetores de traços latentes foram melhor estimadas pelo modelo 2 d2PPSn $(\mathrm{PP}) . \mathrm{Na}$ Figura 5.15 observamos que as estimativas dos parâmetros de discriminação do modelo 2d2PPSn(PP) envidenciaram muito bem a dimensionalidade de um teste cuja estrutura da matrix de discriminação é aproximadamente simples, ou melhor, que o teste bidimensional pode ser considerado como sendo aproximadamente bivariado.

Tabela 5.16 Parâmetros populacionais e estimativas da amostra da distribuição a posteriori sob o modelo 2d2PPSn (PP).

\begin{tabular}{llrlrrrrr}
\hline \hline \multirow{2}{*}{ Parâmetro } & & & & \multicolumn{5}{c}{ Intervalos de quantis } \\
\cline { 5 - 9 } & Verdadeiro & Média & D.P. & $2.5 \%$ & $25 \%$ & $50 \%$ & $75 \%$ & $97.5 \%$ \\
\hline$E\left(\theta_{1}\right)$ & -0.50 & -0.38 & 0.06 & -0.48 & -0.42 & -0.38 & -0.34 & -0.27 \\
$E\left(\theta_{2}\right)$ & 0.50 & 0.33 & 0.09 & 0.17 & 0.27 & 0.33 & 0.39 & 0.49 \\
\hline $\operatorname{Var}\left(\theta_{1}\right)$ & 0.90 & 0.59 & 0.10 & 0.43 & 0.53 & 0.59 & 0.65 & 0.81 \\
$\operatorname{Var}\left(\theta_{2}\right)$ & 1.52 & 2.52 & 0.43 & 1.78 & 2.22 & 2.48 & 2.76 & 3.46 \\
$\operatorname{Cor}\left(\theta_{1}, \theta_{2}\right)$ & 0.56 & 0.62 & 0.05 & 0.51 & 0.58 & 0.62 & 0.65 & 0.71 \\
\hline$\delta_{1}$ & 0.00 & 0.04 & 0.34 & -0.58 & -0.21 & 0.03 & 0.29 & 0.69 \\
$\delta_{2}$ & -1.5 & -1.61 & 0.33 & -2.13 & -1.82 & -1.65 & -1.45 & -0.74 \\
\hline \hline
\end{tabular}

Matos, G. S. 


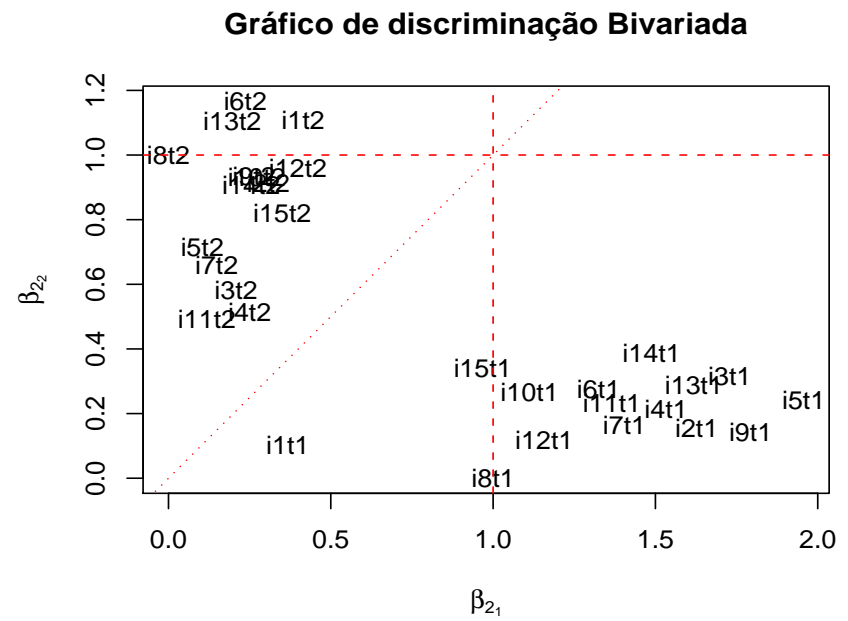

Figura 5.15 Estimativas das discriminações obtidas pelo modelo 2d2PPSn(PP).

Matos, G. S. 


\subsection{Conclusões sobre o Capítulo}

No presente capítulo, dois conjuntos de dados (testes) foram simulados sob a condição de que a verdadeira distribuição dos traços latentes é normal assimétrica. As análises destes conjuntos de dados foram conduzidas sob os modelos de resposta ao item de 2 parâmetros probito da TRI com a especificação a priori de distribuições normais simétricas e assimétricas para os traços latentes de modo que pudéssemos investigar sobre possíveis vantagens ou desvantagens em admitir assimetria na distribuição dos traços latentes. Além disso, procuramos ter uma noção sobre a eficiência do método DAGS com relaçao à estimação dos parâmetros. No que conscerne a este último objetivo podemos dizer que de modo geral o método DAGS estima de maneira satisfatória todos os parâmetros. Isto pode ser pecebido, por exemplo, através dos gráficos das Raízes dos Erros Quadráticos Médio dos itens e desvios ou resíduos dos parâmetros dos itens e traços latentes. É importante apenas lembrar que a observação de alguns valores grandes para os resíduos dos traços latentes encontra justificativa na dificuldade em se construir um teste com itens representantes dos diversos pontos da escala dos traços latentes os quais correspondem aos diversos respondentes de uma determinada população.

As análises desenvolvidas sobre o primeiro conjunto de dados que foi caracterizado segundo um teste do tipo unidimensional (Seção 5.2) revelaram que os modelos 1d2PPN e 1d2PPSn estimaram as características dos itens e dos traços latentes de forma precisa e semelhante (Sub-seção 5.2.4). Na Sub-seção 5.2.5 foi possível observar que apesar de métricas distintas serem induzidas pelos modelos 1d2PPN e 1d2PPSn, interpretações de interesse prático podem ser obtidas de maneira análoga e coerente com as verdadeiras características do teste simulado, desde que as relações de ordem e magnitude entre a média das estimativas médias dos traços latentes e a média das dificuldades estimadas dos itens sejam analisadas. Apesar de termos observado a semelhança entre os modelos estimados no sentido de indicar de maneira análoga o nível de dificuldade de um teste, também foi possível observar que o modelo 1d2PPSn comportou-se de forma mais flexível pois além de corretamente ter fornecido indícios quanto a uma assimetria negativa e moderada também forneceu indícios de que os verdadeiros traços latentes com valores inferiores à -2.2 podem ser melhor estimados. Esta flexibilidade é a provável explicação para o fato de medidas tais como o DIC, EAIC e EBIC indicarem o modelo 1d2PPSn como o que melhor se ajusta aos dados (Seção 5.2.6).

Na Seção 5.3 um teste com uma estrutura aproximadamente simples para a matrix de discriminação dos itens, ou melhor, do tipo aproximadamente bivariado (McDonald, 1999) foi simulado e as análises comparativas entre as estimativas e ajustes dos modelos com distribuições simétricas e assimétricas para os traços latentes não evidenciaram diferenças significativas. De fato, em quase todas as análises, o que foi observado foram apenas leves evidências a favor dos modelos com distribuições assimétricas para os vetores de traços latentes.

Matos, G. S.

$\mathrm{IME} / \mathrm{USP}$ 
Na Sub-seção 5.3.5 argumentamos que para selecionar um modelo com o intuito de divulgar os resultados inferenciais é necessário não apenas levar em consideração os critérios de ajuste e seleção de modelos mas também as questões e objetivos a que se destinam a análise do teste. Levando-se isto em consideração vimos que apenas no caso em que não havia interesse sobre a estimação e interpretação dos parâmetros relacionados à localização e escala da distribuição dos traços latentes é que um modelo com distribuição normal poderia ser selecionado. De fato, todos os critérios de ajuste e seleção de modelo utilizados para este caso indicaram o modelo $2 \mathrm{~d} 2 \mathrm{PPN}$ como o que melhor representa o comportamento dos dados. Por outro lado, quando considerados os modelos que possibilitam a estimação e interpretação dos parâmetros relacionados à localização e escala da distribuição dos traços latentes os resultados favoreceram a seleção dos modelos com distribuições assimétricas.

Um ponto deste capítulo que merece ser comentado refere-se a forma como foram desenvolvidas as análises. Especificamente, queremos dizer que a validade de tais análises podem ser questionadas por não termos desenvolvido estudos adicionais, tais como: análises de sensibilidade à diferentes prioris (em especial às relacionada aos parâmetros populacionais), análises sujeitas à diferentes formas de se identificar os modelos de resposta ao item e/ou análises com o uso de conjunto de dados replicados. Até certo ponto, devemos concordar que tais questionamentos devem ser acatados, porém é necessário dizer que tais análises não foram realizadas por termos dedicado esforços maiores na compreensão e interpretação dos diversos fatores que influenciam o processo de estimação de modelos tão complexos como os aqui analisados. Devemos lembrar que os modelos multidimensionais da TRI do presente trabalho estão sujeitos à diversos tipos de não-identificabilidade (ver Sub-seção 3.3) o que por vezes dificulta a identificação dos fatores que levam à estimativas completamente distintas daquelas esperadas quando os verdadeiros valores dos parâmetros são conhecidos. Nosso relato na Sub-seção 5.3.4.4 é apenas um exemplo das possíveis dificuldades encontradas no desenvolvimento das análises deste capítulo. Na Sub-seção 5.3.3.1 também relatamos nossa experiência e ponto de vista sobre as razões pela qual os parâmetros de assimetria do modelo 2d2PPSn não foram estimados de forma satisfatório. Naquela ocasião, observamos a necessidade de estudos adicionais com o intuito de amenizar o viés relativo ao vetor de assimetria que surge sob determinadas condições específicas. Diante destas circunstâncias e das limitações aqui consideradas, nosso intuito é de que as análises desenvolvidas neste capítulo sirvam como uma boa base para o desenvolvimento de estudos mais aprofundados dos aqui já expostos bem como de estudos que avaliem o desempenho e a aplicabilidade dos modelos não explorados neste capítulo (e.g. t-multivariada assimétrica e FRI's de 3 parâmetros) cujo método de estimação encontra-se desenvolvido no Capítulo 4 deste trabalho.

Matos, G. S.

$\mathrm{IME} / \mathrm{USP}$ 
Capítulo 6

\section{Comentários e Perspectivas para Novas Pesquisas}

No presente trabalho, foram estudados e desenvolvidos alguns modelos multidimensionais da TRI com o intuito de identificar situações particulares em que o uso de uma distribuição normal assimétrica para os traços latentes seja de maior relevância para a estimação e inferência dos parâmetros de itens e de outros relacionados à distribuição dos traços latentes. Conforme foi possível observar, além da complexidade natural dos modelos multidimensionais da TRI, os problemas decorrentes dos vários tipos de não identificabilidade faz com que a estimação, a análise e a interpretação destes modelos sejam tarefas relativamente complexas. Sob este contexto, acreditamos que uma das principais contribuições do presente trabalho seja o desenvolvimento do estudo sobre a falta de identificabilidade tanto sob o paradigma da inferência clássica quanto bayesiana (Sub-seção 3.3). Este estudo bibliográfico revelou-se de grande importância para que as análises e interpretações dos testes simulados no Capítulo 5 do presente trabalho pudessem ser melhor compreendidas e satisfatoriamente realizadas. Como conclusão das análises realizadas no Capítulo 5 podemos dizer que há uma melhora superficial quando a informação sobre uma possível assimetria na distribuição dos traços latentes não é ignorada. Além disso, conforme foi possível observar os resultados favoreceram a seleção dos modelos que consideram distribuições assimétricas para os traços latentes, principalmente quando foram considerados os modelos que possibilitam a estimação dos parâmetros de localização e escala da distribuição dos vetores de traços latentes.

Duas importantes contribuições do presente trabalho que consideramos de ordem prática, são: (i) a análise e a interpretação de testes através da estimação de modelos uni e multidimensionais da TRI que consideram tanto distribuições simétricas quanto assimétricas para os vetores de traços latentes e (ii) a disponibilização de funções escritas em códigos $R$ e $C++$ para a estimação dos modelos aqui apresentados e desenvolvidos (ver Apêndice B). Com relação à contribuição (i), devemos lembrar que nossa experiência evidencia que não somente a forma da distribuição dos traços latentes, se simétrica ou assimétrica, mas também o método e a forma como se resolve a falta de identificabilidade de um modelo da TRI, são fatores que determinam uma particular métrica que pode ou não ser de fácil interpretação. Neste caso, o bom senso e a experiência sobre a necessidade 
de transformar a métrica das estimativas bem como a experiência sobre as circunstâncias em que o conjunto de dados de interesse é obtido são itens imprescindíveis para que o "melhor" resultado seja adotado e divulgado.

Uma das limitações das análises desenvolvidas no Capítulo 5 segue do fato de não termos desenvolvido estudos adicionais, tais como: análises de sensibilidade à diferentes distribuições a priori (em especial às relacionada aos parâmetros populacionais), análises sujeitas à diferentes formas de se identificar os modelos de resposta ao item e/ou análises com o uso de conjunto de dados replicados. Apesar disto, é necessário dizer que tais análises não foram realizadas por termos dedicado esforços maiores na compreensão e interpretação dos diversos fatores que influenciam o processo de estimação de modelos tão complexos como são os multidimensionais da TRI. Neste sentido esperamos que as análises desenvolvidas naquele capítulo sirvam de base para o desenvolvimento de estudos mais aprofundados dos aqui já expostos bem como de estudos que avaliem o desempenho e a aplicabilidade dos modelos não explorados como, por exemplo, os que consideram distribuição t-assimétrica uni e multivariada para os traços latentes e FRI's de 3 parâmetros cujo método de estimação encontra-se desenvolvido no Capítulo 4. Conforme podemos perceber, muitos estudos ainda precisam ser desenvolvidos e alguns que atualmente consideramos de maior relevância são:

\section{Dimensionalidade}

Na Sub-seção 3.3 iniciamos um estudo dizendo que nos modelos multidimensionais da TRI é possível observar que existem pelo menos dois tipos de falta de identificabilidade e naquela ocasião foi dado destaque aos que tem como causa a invariância dos modelos à transformações de localização, escala e rotação. De fato, mais um tipo de nãoidentificabilidade surge quando a matriz de disciminações $\boldsymbol{\beta}_{2}$ é de posto $r$ incompleto, ou seja, posto $\left(\boldsymbol{\beta}_{2}\right)=r<K$. O fato é que sendo $\boldsymbol{\beta}_{2}$ de posto incompleto, existe uma matriz $\mathbf{W}$ tal que $\boldsymbol{\beta}_{2} \mathbf{W}=\mathbf{0}, \mathbf{W}^{\prime} \mathbf{W}=\mathbf{I}$. Sendo assim, observamos que para todo conjunto de dados amostrais, $\boldsymbol{y}$, vale

$$
\begin{aligned}
\Phi\left(-\beta_{1 j}+\boldsymbol{\beta}_{2 j}^{\prime} \boldsymbol{\theta}_{i}\right) & =\Phi\left(-\beta_{1 j}+\boldsymbol{\beta}_{2 j}^{\prime}\left(\boldsymbol{\theta}_{i}+\mathbf{W}\right)\right) \\
& =\Phi\left(-\beta_{1 j}+\boldsymbol{\beta}_{2 j}^{\prime} \boldsymbol{\theta}_{i}^{*}\right)
\end{aligned}
$$

em que $\boldsymbol{\theta}_{i}^{*}=\boldsymbol{\theta}_{i}+\mathbf{W}$.

A falta de identificabilidade devido à deficiência do posto da matriz de discriminações $\boldsymbol{\beta}_{2}$ foi tema abordado por Geweke e Singleton (1980) no contexto da análise fatorial de variáveis contínuas e conforme observado por Lopes e West (2004), esta é uma questão intimamente relacionada com a incerteza sobre o número de fatores (dimensão dos traços latentes em nosso caso) necessários para explicar os fenômenos de interesse (probabilidade de resposta correta em nosso caso). Neste sentido, é de fundamental importância o avanço e/ou a consolidação de métodos que visem identificar a verdadeira dimensão dos vetores de 
traços latentes. Uma proposta para a TRI que toma como base o trabalho de Lopes e West (2004) trata a dimensão de um modelo multidimensional como mais um parâmetro a ser estimado e, neste contexto, inferências sob o paradigma bayesiano podem ser desenvolvidas via algoritmos de Saltos Reversíveis MCMC. Esta proposta encontra-se atualmente em desenvolvimento como tema de tese de Doutorado em Estatística IME-USP (pelo autor Nojosa (2002)).

\section{A Arte dos Dados Aumentados}

O método de estimação dos modelos desenvolvidos neste trabalho utilizou-se de um esquema denominado Dados Aumentados (DA). Este esquema tem por objetivo construir algoritmos de otimização e amostragem mais simples e de maior velocidade computacional via introdução de dados não observados ou variáveis latentes no modelo (Dempster et al., 1977; Tanner; Wong, 1987). Entretanto, conforme van Dyk e Meng (2001) observaram:

" In general, however, constructing data augmentation schemes that result in both simple and fast algorithms is a matter of art in that successful strategies vary greatly with the (observed-data) models being considered."

Em nosso trabalho uma das dificuldades encontradas com o esquema de dados aumentados foi a ocorrência de altas correlações de algumas cadeias marginais de parâmetros e isto ocorreu especialmente nos modelos com distribuição assimétrica, exigindo assim, um tempo considerável de procesamento computacional para que longas Cadeias de Markov pudessem ser obtidas (Sub-seções 5.2.3, 5.3.3.2 e 5.3.4.1).

van Dyk e Meng (2001) após revisarem alguns métodos de construção de dados aumentados, introduziram uma estratégia efetiva de busca por bons esquemas de dados aumentados; combinando idéias sobre aumento marginal e aumento condicional juntamente com um método de aproximação determinística. Estes autores aplicaram a estratégia desenvolvida à algumas classes de modelos comumente encontradas na literatura. Além disso, eles forneceram evidências teóricas e empíricas de que os algoritmos resultantes, enquanto exigem esforços similares de programação, podem apresentar melhorias dramáticas sobre o amostrador de Gibbs comumente utilizado nos modelos por eles analisados. Uma proposta de pesquisa, portanto, é investigar se a metodologia desenvolvida por van Dyk e Meng (2001) pode ou não trazer melhorias para o processo de estimação dos modelos da TRI.

Matos, G. S.

$\mathrm{IME} / \mathrm{USP}$ 


\section{Conclusão}

A Teoria da Resposta ao Item, como podemos ver, constitui um campo fértil para o desenvolvimento de novas pesquisas e por isso poderíamos escrever sobre muitas propostas. No entanto, não repetiremos aqui algumas pesquisas sugeridas, por exemplo, por Tavares (2001) e Azevedo (2008) mas apenas lembramos que as idéias desenvolvidas em nosso trabalho podem ser combinadas com a idéia de também considerar assimetria sobre as funções de resposta ao item; conforme Bazán et al. (2006) desenvolveram para o caso de alguns modelos unidimensionais da TRI. Esta última proposta certamente tornará os modelos mais flexíveis e realísticos mas também de maior complexidade e quem sabe de grande importância prática para a análise de dados reais.

Matos, G. S. 
Apêndice A

\section{Condicionais Completas para o Modelo Kd3PP-tA}

Este apêndice é dedicado à obtenção das distribuições condicionais completas associadas ao modelo Kd3PP-tA. Na próxima seção expressamos alguns resultados de grande utilidade para esta tarefa e nas seções posteriores obtemos as distribuições considerando-se um "descarte" inicial de contantes proporcionais à distribuição a posteriori conjunta (4.6) conforme a condicional completa de interesse.

Lembramos que, por simplicidade, a notação do tipo $\boldsymbol{\tau} \mid \ldots, \boldsymbol{y}$ representa neste trabalho a distribuição condicional a posteriori de um determinado parâmetro $\boldsymbol{\tau}$ qualquer condicionado à todos os demais parâmetros $\boldsymbol{\zeta}$ com exceção do parâmetro de interesse $\boldsymbol{\tau}$. O caso em que o vetor de respostas $\boldsymbol{y}$ não aparece na notação corresponde a uma distribuição condicional completa de interesse que não depende diretamente dos dados (respostas aos itens) ou de qualquer subconjunto dos dados.

\section{A.1 Resultados Úteis}

\section{A.1.1 Soma de Formas Quadráticas}

Se $\boldsymbol{x}, \boldsymbol{a}$ e $\boldsymbol{b}$ são vetores $p \times 1$ e $\boldsymbol{A}$ e $\boldsymbol{B}$ matrizes simétricas positivas definidas tais que $\boldsymbol{A}+\boldsymbol{B}$ é não singular, então a seguinte identidade é válida:

$$
\begin{aligned}
(\boldsymbol{x}-\boldsymbol{a})^{\prime} \boldsymbol{A}(\boldsymbol{x}-\boldsymbol{a})+(\boldsymbol{x}-\boldsymbol{b})^{\prime} \boldsymbol{B}(\boldsymbol{x}-\boldsymbol{b}) & =(\boldsymbol{x}-\boldsymbol{c})^{\prime}(\boldsymbol{A}+\boldsymbol{B})(\boldsymbol{x}-\boldsymbol{c}) \\
& +(\boldsymbol{a}-\boldsymbol{b})^{\prime} \boldsymbol{C}(\boldsymbol{a}-\boldsymbol{b}),
\end{aligned}
$$

onde

$$
\begin{aligned}
& \boldsymbol{c}=(\boldsymbol{A}+\boldsymbol{B})^{-1}(\boldsymbol{A} \boldsymbol{a}+\boldsymbol{B} \boldsymbol{b}) \\
& \boldsymbol{C}=\boldsymbol{A}(\boldsymbol{A}+\boldsymbol{B})^{-1} \boldsymbol{B}=\left(\boldsymbol{A}^{-1}+\boldsymbol{B}^{-1}\right)^{-1}
\end{aligned}
$$

Prova: Ver, por exemplo, Box e Tiao (1973). 


\section{A.1.2 Inversa da Soma de Matrizes}

Note que

$$
(\boldsymbol{A}+\boldsymbol{U} \boldsymbol{B} \boldsymbol{V})^{-1}=\boldsymbol{A}^{-1}-\boldsymbol{A}^{-1} \boldsymbol{U} \boldsymbol{B}\left(\boldsymbol{B}+\boldsymbol{B} \boldsymbol{V} \boldsymbol{A}^{-1} \boldsymbol{U} \boldsymbol{B}\right)^{-1} \boldsymbol{B} \boldsymbol{V} \boldsymbol{A}^{-1},
$$

desde que as inversas existam.

Quando $\boldsymbol{U} \boldsymbol{B}=\boldsymbol{I} \times \boldsymbol{I}$, a igualdade (A.2) resulta na seguinte forma particular:

$$
\begin{aligned}
(\boldsymbol{A}+\boldsymbol{V})^{-1} & =(\boldsymbol{A}+\boldsymbol{I} \times \boldsymbol{I} \boldsymbol{V})^{-1} \\
& =\boldsymbol{A}^{-1}-\boldsymbol{A}^{-1} \boldsymbol{I} \times \boldsymbol{I}\left(\boldsymbol{I}+\boldsymbol{I} \boldsymbol{V} \boldsymbol{A}^{-1} \boldsymbol{I} \times \boldsymbol{I}\right)^{-1} \boldsymbol{I} \boldsymbol{V} \boldsymbol{A}^{-1} \\
& =\boldsymbol{A}^{-1}-\boldsymbol{A}^{-1}\left[\boldsymbol{V}\left(\boldsymbol{V}^{-1}+\boldsymbol{A}^{-1}\right)\right]^{-1} \boldsymbol{V} \boldsymbol{A}^{-1} \\
& =\boldsymbol{A}^{-1}-\boldsymbol{A}^{-1}\left(\boldsymbol{V}^{-1}+\boldsymbol{A}^{-1}\right)^{-1} \boldsymbol{V}^{-1} \boldsymbol{V} \boldsymbol{A}^{-1} \\
& =\boldsymbol{A}^{-1}-\boldsymbol{A}^{-1}\left(\boldsymbol{V}^{-1}+\boldsymbol{A}^{-1}\right)^{-1} A^{-1}
\end{aligned}
$$

Prova: Ver, por exemplo, Seber (1984).

\section{A.1.3 Posteriori do Coeficiente $\beta$ de um Modelo de Regressão Linear Normal/Normal}

Observe que o seguinte modelo de regressão linear normal

$$
\begin{aligned}
y_{i} & =\boldsymbol{X}_{i}^{\prime} \boldsymbol{\beta}+\epsilon_{i} \\
\epsilon_{i} & \sim N\left(0, \sigma^{2}\right), \quad i=1, \ldots, N \quad \operatorname{com} \sigma^{2} \text { conhecido }
\end{aligned}
$$

pode ser escrito na forma matricial como

$$
\begin{aligned}
\boldsymbol{y} & =\boldsymbol{X} \boldsymbol{\beta}+\boldsymbol{\epsilon} \\
\boldsymbol{\epsilon} & \sim N_{N}\left(\mathbf{0}, \sigma^{2} \boldsymbol{I}_{N}\right)
\end{aligned}
$$

com $\sigma^{2}$ conhecido e $\boldsymbol{X}$ sendo uma matriz $N \times p$ cuja $i$-ésima linha é $\boldsymbol{X}_{i}^{\prime}$.

Agora, se

$$
\boldsymbol{\beta} \sim N_{p}\left(\boldsymbol{\beta}_{0}, \boldsymbol{\Sigma}_{0}\right) .
$$

Segue que o modelo de regressão linear normal/normal admite a seguinte posteriori para $\beta$ :

$$
\boldsymbol{\beta}|\boldsymbol{y} \stackrel{\text { dist. }}{=} \boldsymbol{\beta}| \widehat{\boldsymbol{\beta}} \sim N_{p}\left(\boldsymbol{\mu}_{\boldsymbol{\beta}}, \boldsymbol{\Sigma}_{\boldsymbol{\beta}}\right)
$$

Matos, G. S. 
em que:

$$
\begin{aligned}
& \boldsymbol{\mu}_{\boldsymbol{\beta}}=\boldsymbol{\mu}_{0}+\boldsymbol{\Sigma}_{0}\left(\boldsymbol{\Sigma}_{0}+\left(\boldsymbol{X}^{\prime} \frac{1}{\sigma^{2}} \boldsymbol{X}\right)^{-1}\right)^{-1}\left(\widehat{\boldsymbol{\beta}}-\boldsymbol{\mu}_{0}\right), \mathrm{e} \\
& \boldsymbol{\Sigma}_{\boldsymbol{\beta}}=\boldsymbol{\Sigma}_{0}-\boldsymbol{\Sigma}_{0}\left(\boldsymbol{\Sigma}_{0}+\left(\boldsymbol{X}^{\prime} \frac{1}{\sigma^{2}} \boldsymbol{X}\right)^{-1}\right)^{-1} \boldsymbol{\Sigma}_{0}
\end{aligned}
$$

$\operatorname{com} \widehat{\boldsymbol{\beta}}=\left(\boldsymbol{X}^{\prime} \frac{1}{\sigma^{2}} \boldsymbol{X}\right)^{-1} \boldsymbol{X}^{\prime} \frac{1}{\sigma^{2}} \boldsymbol{y}$, o estimador de máxima verossimilhança (EMV).

Note que a distribuição a posteriori (A.6) ainda pode ser reescrita como:

$$
\boldsymbol{\beta}|\boldsymbol{y}=\boldsymbol{\beta}| \widehat{\boldsymbol{\beta}} \sim N_{p}\left(\boldsymbol{\Psi}\left[\boldsymbol{\Sigma}_{0}^{-1} \boldsymbol{\mu}_{0}+\left(\boldsymbol{X}^{\prime} \frac{1}{\sigma^{2}} \boldsymbol{X}\right) \widehat{\boldsymbol{\beta}}\right], \Psi\right)
$$

ou

$$
\boldsymbol{\beta}|\boldsymbol{y}=\boldsymbol{\beta}| \widehat{\boldsymbol{\beta}} \sim N_{p}\left(\boldsymbol{\Psi}\left[\boldsymbol{\Sigma}_{0}^{-1} \boldsymbol{\mu}_{0}+\boldsymbol{X}^{\prime} \frac{1}{\sigma^{2}} \boldsymbol{y}\right], \boldsymbol{\Psi}\right)
$$

$\operatorname{com} \Psi=\left(\boldsymbol{\Sigma}_{0}^{-1}+\boldsymbol{X}^{\prime} \frac{1}{\sigma^{2}} \boldsymbol{X}\right)^{-1}$.

Prova: Para obter a distribuição a posteriori expressa por (A.6) é necessário reescrever a verossimilhança do modelo (A.4) em função de um vetor de estatísticas suficientes para o parâmetro $\beta$, em seguida reescrever a verossimilhança em função do estimador de máxima verossimilhança $\widehat{\beta}$, multiplicando assim a distribuição a priori (A.5) pela verossimilhança resultante. O resultado segue após algumas manipulações algébricas e o uso do resultado (A.1).

Para verificar a equivalência entre as distribuições a posteriori (A.6) e (A.7) faz-se necessário o uso do resultado (A.3), juntamente com algumas manipulações algébricas. 


\section{A.2 Condicional completa de $Q=\Sigma_{\theta}^{-1}$}

Uma vez fixado todas as variáveis do modelo Kd3PP-tA com exceção da variável $\boldsymbol{Q}$, a densidade da condicional completa de $\boldsymbol{Q}=\boldsymbol{\Sigma}_{\theta}^{-1}$ fica proporcional a

$$
\begin{aligned}
\pi\left(\boldsymbol{\Sigma}_{\theta}^{-1} \mid \ldots, \boldsymbol{y}\right) & \propto \prod_{i=1}^{N}\left|\frac{\boldsymbol{\Sigma}_{\theta}}{\omega_{i}}\right|^{-1 / 2} \exp \left\{-\frac{1}{2}\left(\boldsymbol{\theta}_{i}-\boldsymbol{\mu}_{\theta}-\boldsymbol{D} \boldsymbol{z}_{i}\right)^{\prime}\left(\frac{\boldsymbol{\Sigma}_{\theta}}{\omega_{i}}\right)^{-1}\left(\boldsymbol{\theta}_{i}-\boldsymbol{\mu}_{\theta}-\boldsymbol{D} \boldsymbol{z}_{i}\right)\right\} \\
& \times|\boldsymbol{Q}|^{\frac{1}{2}(2 r-K-1)} \exp \left\{-\frac{1}{2} \operatorname{tr}\left(\frac{1}{2} \boldsymbol{\Sigma}_{Q}^{-1} \boldsymbol{Q}\right)\right\} \\
& \propto|\boldsymbol{Q}|^{N / 2}|\boldsymbol{Q}|^{\frac{1}{2}(2 r-K-1)} \\
& \times \exp \left\{-\frac{1}{2}\left\{\operatorname{tr}\left[\sum_{i=1}^{N}\left\{\left(\boldsymbol{\theta}_{i}-\boldsymbol{\mu}_{\theta}-\boldsymbol{D} \boldsymbol{z}_{i}\right)\left(\boldsymbol{\theta}_{i}-\boldsymbol{\mu}_{\theta}-\boldsymbol{D} \boldsymbol{z}_{i}\right)^{\prime} \omega_{i}\right\} \boldsymbol{Q}\right]\right\}\right. \\
& =|\boldsymbol{Q}|^{\frac{1}{2}[(2 r+N)-K-1]} \times \exp \left\{-\frac{1}{2} \operatorname{tr}\left[\boldsymbol{S}^{-1} \boldsymbol{Q}\right]\right\}
\end{aligned}
$$

em que:

$$
\boldsymbol{S}=\left\{\sum_{i=1}^{N}\left\{\omega_{i}\left(\boldsymbol{\theta}_{i}-\boldsymbol{\mu}_{\theta}-\boldsymbol{D} \boldsymbol{z}_{i}\right)\left(\boldsymbol{\theta}_{i}-\boldsymbol{\mu}_{\theta}-\boldsymbol{D} \boldsymbol{z}_{i}\right)^{\prime}\right\}+\frac{1}{2} \boldsymbol{\Sigma}_{Q}^{-1}\right\}^{-1} .
$$

Segue assim que $\boldsymbol{Q}=\boldsymbol{\Sigma}_{\theta}^{-1} \mid \ldots \sim W_{K}(2 r+N, \boldsymbol{S})$ com $\boldsymbol{S}$ expresso por (A.10).

\section{A.3 Condicional Completa de $\mu_{\theta}$}

Neste caso, a densidade condicional a posteriori de $\boldsymbol{\mu}_{\theta}$ é tal que

$$
\begin{aligned}
\pi\left(\boldsymbol{\mu}_{\theta} \mid \ldots, \boldsymbol{y}\right) & \propto \prod_{i=1}^{N}\left|\frac{\boldsymbol{\Sigma}_{\theta}}{\omega_{i}}\right|^{-1 / 2} \exp \left\{-\frac{1}{2}\left(\boldsymbol{\theta}_{i}-\boldsymbol{\mu}_{\theta}-\boldsymbol{D} \boldsymbol{z}_{i}\right)^{\prime}\left(\frac{\boldsymbol{\Sigma}_{\theta}}{\omega_{i}}\right)^{-1}\left(\boldsymbol{\theta}_{i}-\boldsymbol{\mu}_{\theta}-\boldsymbol{D} \boldsymbol{z}_{i}\right)\right\} \\
& \times \exp \left\{-\frac{1}{2}\left(\boldsymbol{\mu}_{\theta}-\boldsymbol{\mu}_{0}\right)^{\prime} \boldsymbol{\Lambda}_{0}^{-1}\left(\boldsymbol{\mu}_{\theta}-\boldsymbol{\mu}_{0}\right)\right\} \\
& \propto \exp \left\{-\frac{1}{2} \sum_{i=1}^{N}\left(\boldsymbol{\mu}_{\theta}-\boldsymbol{C}_{i}\right)^{\prime} \omega_{i} \boldsymbol{Q}\left(\boldsymbol{\mu}_{\theta}-\boldsymbol{C}_{i}\right)\right\} \\
& \times \exp \left\{-\frac{1}{2}\left(\boldsymbol{\mu}_{\theta}-\boldsymbol{\mu}_{0}\right)^{\prime} \boldsymbol{\Lambda}_{0}^{-1}\left(\boldsymbol{\mu}_{\theta}-\boldsymbol{\mu}_{0}\right)\right\}
\end{aligned}
$$

onde

$$
\boldsymbol{C}_{i}=\boldsymbol{\theta}_{i}-\boldsymbol{D} \boldsymbol{z}_{i}
$$

Matos, G. S. 
Agora note que

$$
\begin{aligned}
\sum_{i=1}^{N}\left(\boldsymbol{\mu}_{\theta}-\boldsymbol{C}_{i}\right)^{\prime} \omega_{i} \boldsymbol{Q}\left(\boldsymbol{\mu}_{\theta}-\boldsymbol{C}_{i}\right) & =\text { Const. }+\boldsymbol{\mu}_{\theta}^{\prime} N \boldsymbol{Q} \bar{\omega} \boldsymbol{\mu}_{\theta}-\boldsymbol{\mu}_{\theta}^{\prime} N \boldsymbol{Q} \overline{\boldsymbol{E}}-\overline{\boldsymbol{E}}^{\prime} N \boldsymbol{Q} \boldsymbol{\mu}_{\theta} \\
& =\text { Const. }+\boldsymbol{\mu}_{\theta}^{\prime} N \boldsymbol{Q} \bar{\omega} \boldsymbol{\mu}_{\theta} \\
& -\boldsymbol{\mu}_{\theta}^{\prime} N \boldsymbol{Q} \overline{\bar{\omega}} \frac{\overline{\boldsymbol{E}}}{\bar{\omega}}-\frac{\overline{\boldsymbol{E}}^{\prime}}{\bar{\omega}} N \boldsymbol{Q} \bar{\omega} \boldsymbol{\mu}_{\theta} \\
\text { emque } & +\frac{\overline{\boldsymbol{E}}^{\prime}}{\bar{\omega}} N \boldsymbol{Q} \overline{\bar{\omega}} \frac{\overline{\boldsymbol{E}}}{\bar{\omega}} \\
& =\left(\boldsymbol{\mu}_{\theta}-\frac{\overline{\boldsymbol{E}}}{\bar{\omega}}\right)^{\prime} N \boldsymbol{Q} \bar{\omega}\left(\boldsymbol{\mu}_{\theta}-\frac{\overline{\boldsymbol{E}}}{\bar{\omega}}\right) .
\end{aligned}
$$

Após substituir (A.12) em (A.11) e usar o resultado (A.1) é possível concluir que

$$
\boldsymbol{\mu}_{\theta} \mid \ldots \sim N_{K}\left(\boldsymbol{W}^{-1} \boldsymbol{b}, \boldsymbol{W}^{-1}\right)
$$

onde:

- $\boldsymbol{W}=N \boldsymbol{Q} \bar{\omega}+\boldsymbol{\Lambda}_{0}^{-1} \quad \operatorname{com} \quad \boldsymbol{Q}=\boldsymbol{\Sigma}_{\theta}^{-1} \quad$ e $\quad \bar{\omega}=\frac{\sum_{i=1}^{N} \omega_{i}}{N}$

- $\boldsymbol{b}=N \boldsymbol{Q} \overline{\boldsymbol{E}}+\boldsymbol{\Lambda}_{0}^{-1} \boldsymbol{\mu}_{0}, \quad \mathrm{com}$

$$
-\bar{E}=\frac{\sum_{i=1}^{N} \boldsymbol{C}_{i} \omega_{i}}{N} \quad \text { em que } \quad \boldsymbol{C}_{i}=\left(\boldsymbol{\theta}_{i}-\boldsymbol{D} \boldsymbol{z}_{i}\right)
$$

Em particular, quando $\boldsymbol{\theta}_{1}, \ldots, \boldsymbol{\theta}_{N}$ são provenientes de uma distribuição Normal Assimétrica, tem-se:

- $\boldsymbol{W}=N \boldsymbol{Q}+\boldsymbol{\Lambda}_{0}^{-1} \quad$ e $\quad \boldsymbol{b}=N \boldsymbol{Q} \overline{\boldsymbol{C}}+\boldsymbol{\Lambda}_{0}^{-1} \boldsymbol{\mu}_{0} . \quad$ Pois, $\quad \bar{\omega}=\frac{\sum_{i=1}^{N} 1}{N}=1$ e neste caso

$$
-\bar{E}=\bar{C}=\frac{\sum_{i=1}^{N} \boldsymbol{C}_{i}}{N} \quad \text { com } \quad \boldsymbol{C}_{i}=\left(\boldsymbol{\theta}_{i}-\boldsymbol{D} \boldsymbol{z}_{i}\right)
$$

No caso em que $\boldsymbol{\theta}_{1}, \ldots, \boldsymbol{\theta}_{N}$ são provenientes de uma distribuição Normal Simétrica, tem-se $\omega_{i}=1, \forall i$ e $\boldsymbol{D}=\mathbf{0}$. Segue, portanto, que:

- $\boldsymbol{W}=N \boldsymbol{Q}+\boldsymbol{\Lambda}_{0}^{-1} \quad$ e $\quad \boldsymbol{b}=N \boldsymbol{Q} \overline{\boldsymbol{\theta}}+\boldsymbol{\Lambda}_{0}^{-1} \boldsymbol{\mu}_{0}$. 


\section{A.4 Condicional Completa de $\left(U_{i j}, V_{i j}\right)^{\prime}$}

Para obter a distribuição condicional completa de cada vetor aleatório $\left(U_{i j}, V_{i j}\right)^{\prime}$, simplesmente consideramos os termos de interesse (não constantes) da posteriori (4.6). Além disso, só devemos considerar a distribuição condicional completa de $\left(U_{i j}, V_{i j}\right)^{\prime}$ para aqueles elementos cuja variável aleatória observada $m_{i j}$ assume o valor 1 , ou seja, em que o item $j$ é submetido ao $i$-ésimo indivíduo (veja discussão na página 8). Neste caso, a distribuição condicional é tal que

$$
\pi\left(\left(U_{i j}, V_{i j}\right)^{\prime} \mid \ldots, y_{i j}, m_{i j}=1\right) \propto \beta_{3 j}^{u_{i j}}\left(1-\beta_{3 j}\right)^{1-u_{i j}} \exp \left\{-\frac{1}{2}\left(v_{i j}-\eta_{i j}\right)^{2}\right\} \boldsymbol{I}\left(y_{i j}, u_{i j}, v_{i j}\right),
$$

em que $\eta_{i j}=\boldsymbol{\Theta}_{i}^{\prime} \boldsymbol{\beta}_{12 j}$, com $\boldsymbol{\Theta}_{i}^{\prime}=\left(-1, \boldsymbol{\theta}_{i}^{\prime}\right)$ vetor $1 \times(1+K)$ e $\boldsymbol{\beta}_{12 j}=\left(\beta_{1 j}, \boldsymbol{\beta}_{2 j}^{\prime}\right)^{\prime} . \boldsymbol{I}\left(y_{i j}, u_{i j}, v_{i j}\right)$ é a função indicadora definida na Proposição (4.2.1).

Observe que para a análise de dados através do modelo de resposta ao item de 2 ou 1 parâmetro probito, o parâmetro de acerto casual estará ausente, ou melhor, $\beta_{3 j}=0$ na FRI e, neste caso, não existirá a variável latente auxiliar $U_{i j}$ na verossimilhança de dados aumentados. Conseqüentemente, uma distribuição normal truncada univariada para cada condicional completa de $V_{i j}$ é obtida, tal como apresentada por Albert (1992).

\section{A.5 Condicionais Completas de $\boldsymbol{Z}=\left(\boldsymbol{Z}_{1}, \ldots, \boldsymbol{Z}_{N}\right)$}

$$
\begin{aligned}
\pi(\boldsymbol{Z} \mid \ldots, \boldsymbol{y}) & \propto \prod_{i=1}^{N} \exp \left\{-\frac{1}{2}\left(\boldsymbol{\theta}_{i}-\boldsymbol{\mu}_{\theta}-\boldsymbol{D} \boldsymbol{z}_{i}\right)^{\prime}\left(\frac{\boldsymbol{\Sigma}_{\theta}}{\omega_{i}}\right)^{-1}\left(\boldsymbol{\theta}_{i}-\boldsymbol{\mu}_{\theta}-\boldsymbol{D} \boldsymbol{z}_{i}\right)\right\} \\
& \times \prod_{i=1}^{N} \exp \left\{-\frac{1}{2} \boldsymbol{z}_{i}^{\prime} \boldsymbol{z}_{i}\right\} I\left(\boldsymbol{z}_{i}>\mathbf{0}\right) \\
& =\prod_{i=1}^{N} \exp \left\{-\frac{1}{2}\left(\boldsymbol{z}_{i}-\boldsymbol{D}^{-1}\left(\boldsymbol{\theta}_{i}-\boldsymbol{\mu}_{\theta}\right)\right)^{\prime} \boldsymbol{D}^{\prime}\left(\frac{\boldsymbol{\Sigma}_{\theta}}{\omega_{i}}\right)^{-1} \boldsymbol{D}\left(\boldsymbol{z}_{i}-\boldsymbol{D}^{-1}\left(\boldsymbol{\theta}_{i}-\boldsymbol{\mu}_{\theta}\right)\right)\right\} \\
& \times \exp \left\{-\frac{1}{2} \boldsymbol{z}_{i}^{\prime} \boldsymbol{z}_{i}\right\} I\left(\boldsymbol{z}_{i}>\mathbf{0}\right) \\
& \propto \prod_{i=1}^{N} \exp \left\{-\frac{1}{2}\left(\boldsymbol{z}_{i}-\boldsymbol{A}_{i}^{-1} \boldsymbol{a}_{i}\right)^{\prime} \boldsymbol{A}_{i}\left(\boldsymbol{z}_{i}-\boldsymbol{A}_{i}^{-1} \boldsymbol{a}_{i}\right)\right\} I\left(\boldsymbol{z}_{i}>\mathbf{0}\right),
\end{aligned}
$$

onde a última linha segue após utilizar o resultado (A.1) com $\boldsymbol{A}_{i}$ e $\boldsymbol{a}_{i}$ dados a seguir. Conseqüentemente, nota-se que

$$
\boldsymbol{Z}_{i} \mid \ldots \stackrel{i n d .}{\sim} N_{K}\left(\boldsymbol{A}_{i}^{-1} \boldsymbol{a}_{i}, \boldsymbol{A}_{i}^{-1}\right) I\left(\boldsymbol{z}_{i}>\mathbf{0}\right), \quad i=1, \ldots, N
$$

Matos, G. S. 
onde

$$
\boldsymbol{A}_{i}=\omega_{i} \boldsymbol{D Q D}+\boldsymbol{I} \quad \text { e } \quad \boldsymbol{a}_{i}=\omega_{i} \boldsymbol{D} \boldsymbol{Q}\left(\boldsymbol{\theta}_{i}-\boldsymbol{\mu}_{\theta}\right) .
$$

\section{A.6 Condicional Completa de $\boldsymbol{\theta}=\left(\boldsymbol{\theta}_{1}^{\prime}, \ldots, \boldsymbol{\theta}_{N}^{\prime}\right)$}

Para obter a distribuição condicional completa associada a um determinado traço latente $\boldsymbol{\theta}_{i}$ devemos notar que a informação a priori sobre este traço só será atualizada por aqueles fatores da verossimilhança cujas variáveis $m_{i j}$ são iguais a 1, ou seja, somente os itens submetidos ao $i$-ésimo respondente é que fornecem informação adicional sobre o correspondente traço latente. Deste modo, segue que

$$
\begin{aligned}
\pi\left(\boldsymbol{\theta}_{i} \mid \ldots, y_{i j}, m_{i j}\right) & \propto \prod_{j=1}^{J}\left\{\exp \left\{-\frac{1}{2}\left(v_{i j}-\boldsymbol{\Theta}_{i}^{\prime} \boldsymbol{\beta}_{12 j}\right)^{2}\right\} \boldsymbol{I}\left(y_{i j}, u_{i j}, v_{i j}\right)\right\}^{m_{i j}} \\
& \times \exp \left\{-\frac{1}{2}\left(\boldsymbol{\theta}_{i}-\left(\boldsymbol{\mu}_{\theta}+\boldsymbol{D} \boldsymbol{z}_{i}\right)\right)^{\prime}\left(\frac{\boldsymbol{\Sigma}_{\theta}}{\omega_{i}}\right)^{-1}\left(\boldsymbol{\theta}_{i}-\left(\boldsymbol{\mu}_{\theta}+\boldsymbol{D} \boldsymbol{z}_{i}\right)\right)\right\} .
\end{aligned}
$$

Ou melhor,

$$
\begin{aligned}
\pi\left(\boldsymbol{\theta}_{i} \mid \ldots, y_{i j}, m_{i j}=1\right) & \propto \prod_{j \in \boldsymbol{J}_{i}} \exp \left\{-\frac{1}{2}\left[v_{i j}-\left(-\beta_{1 j}+\boldsymbol{\theta}_{i}^{\prime} \boldsymbol{\beta}_{2 j}\right)\right]^{2}\right\} \\
& \times \exp \left\{-\frac{1}{2}\left(\boldsymbol{\theta}_{i}-\left(\boldsymbol{\mu}_{\theta}+\boldsymbol{D} \boldsymbol{z}_{i}\right)\right)^{\prime} \omega_{i} \boldsymbol{Q}\left(\boldsymbol{\theta}_{i}-\left(\boldsymbol{\mu}_{\theta}+\boldsymbol{D} \boldsymbol{z}_{i}\right)\right)^{\prime}\right\}
\end{aligned}
$$

em que $\boldsymbol{J}_{i}$ é o conjunto dos índices dos itens submetidos ao $i$-ésimo respondente.

Agora, observe que a variável latente auxiliar $V_{i j}$ admite a seguinte representação hierárquica

$$
\begin{aligned}
v_{i j} & =-\beta_{1 j}+\boldsymbol{\beta}_{2 j}^{\prime} \boldsymbol{\theta}_{i}+\epsilon_{j} \\
\epsilon_{j} & \sim N\left(0, \sigma^{2}\right), \quad j \in \boldsymbol{J}_{i} \quad \operatorname{com} \sigma^{2}=1 .
\end{aligned}
$$

Observe também que devido a distribuição ser condicional, a representação hierárquica pode ser reescrita como:

$$
\begin{aligned}
v_{i j}^{*} & =\boldsymbol{\beta}_{2 j}^{\prime} \boldsymbol{\theta}_{i}+\epsilon_{j} \\
\epsilon_{j} & \sim N\left(0, \sigma^{2}\right), \quad j \in \boldsymbol{J}_{i} \quad \operatorname{com} \sigma^{2}=1,
\end{aligned}
$$

onde $v_{i j}^{*}=v_{i j}+\beta_{1 j}$.

$\mathrm{Na}$ forma matricial, tem-se

$$
\begin{aligned}
\boldsymbol{v}_{i}^{*} & =\boldsymbol{\beta}_{2 .}^{(i)} \boldsymbol{\theta}_{i}+\boldsymbol{\epsilon}_{i}^{*} \\
\boldsymbol{\epsilon}_{i}^{*} & \sim N_{n_{i}}\left(\mathbf{0}, \sigma^{2} \boldsymbol{I}_{n_{i}}\right)
\end{aligned}
$$

Matos, G. S. 
com $\sigma^{2}=1, n_{i}=\operatorname{card}\left\{\boldsymbol{J}_{i}\right\}$ a cardinalidade do conjunto $\boldsymbol{J}_{i}$ e $\boldsymbol{\beta}_{2}^{(i)}$ sendo uma matriz $n_{i} \times K$ cuja $j$-ésima linha corresponde aos parâmetros de discrimação $\boldsymbol{\beta}_{2 j}^{\prime}$ do $j$-ésimo item submetido ao $i$-ésimo respondente.

Agora, após combinar a distribuição a priori

$$
\boldsymbol{\theta}_{i} \mid \boldsymbol{z}_{i}, \boldsymbol{\mu}_{\theta}, \boldsymbol{\Sigma}_{\theta}, \boldsymbol{D}, \omega_{i} \sim N_{K}\left(\boldsymbol{\mu}_{\theta}+\boldsymbol{D} \boldsymbol{Z}_{i}, \frac{\boldsymbol{\Sigma}_{\theta}}{\omega_{i}}\right)
$$

com a verossimilhança (A.16) e adequadamente aplicar o resultado (A.8) é possível concluir que

$$
\boldsymbol{\theta}_{i}\left|\ldots, \boldsymbol{y}_{i}=\boldsymbol{\theta}_{i}\right| \ldots \sim N_{K}\left(\boldsymbol{F}_{i} \boldsymbol{f}_{i}, \boldsymbol{F}_{i}\right)
$$

com

$$
\begin{aligned}
-\boldsymbol{F}_{i} & =\left(\left(\frac{\boldsymbol{\Sigma}_{\theta}}{\omega_{i}}\right)^{-1}+\boldsymbol{\beta}_{2 .}^{(i)^{\prime}} \frac{1}{\sigma^{2}} \boldsymbol{\beta}_{2 .}^{(i)}\right)^{-1}=\left(\omega_{i} \boldsymbol{Q}+\boldsymbol{\beta}_{2 .}^{(i)^{\prime}} \frac{1}{\sigma^{2}} \boldsymbol{\beta}_{2 .}^{(i)}\right)^{-1} \mathrm{e} \\
-\boldsymbol{f}_{i} & =\left(\frac{\boldsymbol{\Sigma}_{\theta}}{\omega_{i}}\right)^{-1}\left(\boldsymbol{\mu}_{\theta}+\boldsymbol{D} \boldsymbol{Z}_{i}\right)+\boldsymbol{\beta}_{2 .}^{(i)^{\prime}} \frac{1}{\sigma^{2}} \boldsymbol{v}_{i}^{*} \\
& =\omega_{i} \boldsymbol{Q}\left(\boldsymbol{\mu}_{\theta}+\boldsymbol{D} \boldsymbol{Z}_{i}\right)+\boldsymbol{\beta}_{2 .}^{(i)^{\prime}} \frac{1}{\sigma^{2}} \boldsymbol{v}_{i}^{*} .
\end{aligned}
$$

Onde $\sigma^{2}=1$.

\section{A.7 Condicional Completa de $\boldsymbol{\beta}_{12 j}=\left(\beta_{1 j}, \boldsymbol{\beta}_{2 j}^{\prime}\right)^{\prime}$}

A distribuição condicional completa associada a cada vetor de parâmetros do $j$-ésimo item pode ser obtida de maneira análoga ao traço latente $\boldsymbol{\theta}_{i}$. Neste sentido, note que

$$
\begin{aligned}
\pi\left(\boldsymbol{\beta}_{12 j} \mid \ldots, y_{i j}, m_{i j}=1\right) & \propto \prod_{i \in \boldsymbol{I}_{j}} \exp \left\{-\frac{1}{2}\left[v_{i j}-\boldsymbol{\Theta}_{i}^{\prime} \boldsymbol{\beta}_{12 j}\right]^{2}\right\} \\
& \times \exp \left\{-\frac{1}{2}\left(\boldsymbol{\beta}_{12 j}-\boldsymbol{b}_{0, j}\right)^{\prime} \boldsymbol{B}_{0, j}^{-1}\left(\boldsymbol{\beta}_{12 j}-\boldsymbol{b}_{0, j}\right),\right.
\end{aligned}
$$

onde $\boldsymbol{I}_{j}$ é o conjunto dos índices dos respondentes que foram apresentados ao $j$-ésimo item e $\boldsymbol{\Theta}_{i}^{\prime}=\left(-1, \boldsymbol{\theta}_{i}^{\prime}\right), i \in \boldsymbol{I}_{j}$ é um vetor $1 \times(1+K)$.

Agora, observe que a variável latente auxiliar $V_{i j}$ admite a seguinte representação hierárquica

$$
\begin{aligned}
v_{i j} & =\boldsymbol{\Theta}_{i}^{\prime} \boldsymbol{\beta}_{12 j}+\epsilon_{i} \\
\epsilon_{i} & \sim N\left(0, \sigma^{2}\right), \quad i \in \boldsymbol{I}_{j} \quad \text { com } \sigma^{2}=1 .
\end{aligned}
$$

Matos, G. S. 
$\mathrm{Na}$ forma matricial

$$
\begin{aligned}
& \boldsymbol{v}_{j}=\boldsymbol{\Theta}^{(j)} \boldsymbol{\beta}_{12 j}+\boldsymbol{\epsilon}_{j} \\
& \boldsymbol{\epsilon}_{j} \sim N_{n_{j}}\left(\mathbf{0}, \sigma^{2} \boldsymbol{I}_{n_{j}}\right),
\end{aligned}
$$

em que $\boldsymbol{v}_{j}=\left(v_{1 j}, \ldots, v_{i j}, \ldots, v_{n_{j}}\right)^{\prime}, \quad i \in \boldsymbol{I}_{j} \operatorname{com} n_{j}=\operatorname{card}\left\{\boldsymbol{I}_{j}\right\}$ representando a cardinalidade do conjunto $\boldsymbol{I}_{j}, \sigma^{2}=1$ e $\boldsymbol{\Theta}^{(j)}$ é uma matriz $n_{j} \times K$ cuja $i$-ésima linha é dada por $\boldsymbol{\Theta}_{i}^{\prime}=\left(-1, \boldsymbol{\theta}_{i}^{\prime}\right), \quad i \in \boldsymbol{I}_{j}$.

Agora, após combinar a distribuição a priori

$$
\boldsymbol{\beta}_{12 j}=\left(\beta_{1 j}, \boldsymbol{\beta}_{2 j}^{\prime}\right)^{\prime} \sim N_{1+K}\left(\boldsymbol{b}_{0, j}, \boldsymbol{B}_{0, j}\right)
$$

com a verossimilhança associada ao modelo (A.18) e adequadamente aplicar o resultado (A.8) é possível concluir que

$$
\boldsymbol{\beta}_{12 j}\left|\ldots, \boldsymbol{y}=\boldsymbol{\beta}_{12 j}\right| \ldots \sim N_{1+K}\left(\boldsymbol{\varphi}_{j} \boldsymbol{m}_{j}, \boldsymbol{\varphi}_{j}\right),
$$

onde $\boldsymbol{\varphi}_{j}=\left(\boldsymbol{B}_{0, j}^{-1}+\boldsymbol{\Theta}^{(j)^{\prime}} \boldsymbol{\Theta}^{(j)}\right)^{-1}$ e $\quad \boldsymbol{m}_{j}=\left(\boldsymbol{B}_{0, j}^{-1} \boldsymbol{b}_{0, j}+\boldsymbol{\Theta}^{(j)^{\prime}} \boldsymbol{v}_{j}\right)$

\section{A.8 Condicional Completa de $\beta_{3 j}$}

A partir da densidade a posteriori (4.6) observa-se que para todo $j=1, \ldots, J$ :

$$
\pi\left(\beta_{3 j} \mid \ldots, y_{i j}, m_{i j}\right) \propto \prod_{i=1}^{N}\left\{\beta_{3 j}^{u_{i j}}\left(1-\beta_{3 j}\right)^{1-u_{i j}}\right\}^{m_{i j}} \times\left(\beta_{3 j}\right)^{a_{j}-1}\left(1-\beta_{3 j}\right)^{b_{j}-1}
$$

Agora, seguindo idéias e notações análogas as discutidas na seção anterior obtemos

$$
\pi\left(\beta_{3 j} \mid \ldots, y_{i j}, m_{i j}=1\right) \propto \beta_{3 j}^{u . j+a_{j}-1}\left(1-\beta_{3 j}\right)^{n_{j}-u_{. j}+b_{j}-1},
$$

onde $u_{\cdot j}=\sum_{i \in \boldsymbol{I}_{j}} u_{i j}, \quad$ e $\quad a_{j}, b_{j}$ são os hiperparâmetros de $\beta_{3 j}$.

\section{A.9 Condicional Completa de $\omega_{i}, \quad i=1, \ldots, N$}

$$
\begin{aligned}
\pi\left(\omega_{i} \mid \ldots, \boldsymbol{y}\right) & \propto\left|\frac{\boldsymbol{\Sigma}_{\theta}}{\omega_{i}}\right|^{-1 / 2} \exp \left\{-\frac{1}{2}\left(\boldsymbol{\theta}_{i}-\boldsymbol{\mu}_{\theta}-\boldsymbol{D} \boldsymbol{z}_{i}\right)^{\prime} \omega_{i} \boldsymbol{Q}\left(\boldsymbol{\theta}_{i}-\boldsymbol{\mu}_{\theta}-\boldsymbol{D} \boldsymbol{z}_{i}\right)\right\} \\
& \times \omega_{i}^{\frac{\nu / 2}{2}-1} \exp \left\{-\frac{1}{2}\left(\frac{2}{\nu}\right) \omega_{i}\right\} I\left(\omega_{i}>0\right) \\
& =\omega_{i}^{\frac{K+\nu / 2}{2}-1} \exp \left\{-\frac{1}{2}\left[\left(\boldsymbol{\theta}_{i}-\boldsymbol{\mu}_{\theta}-\boldsymbol{D} \boldsymbol{z}_{i}\right)^{\prime} \boldsymbol{Q}\left(\boldsymbol{\theta}_{i}-\boldsymbol{\mu}_{\theta}-\boldsymbol{D} \boldsymbol{z}_{i}\right)+\frac{2}{\nu}\right] \omega_{i}\right\} I\left(\omega_{i}>0\right) .
\end{aligned}
$$

Matos, G. S. 
Logo,

$$
\omega_{i} \mid \ldots \sim \operatorname{Gama}\left(\frac{K+\nu / 2}{2}, \frac{c_{i}}{2}\right)
$$

em que

$$
c_{i}=2\left\{\left(\boldsymbol{\theta}_{i}-\boldsymbol{\mu}_{\theta}-\boldsymbol{D} \boldsymbol{z}_{i}\right)^{\prime} \boldsymbol{Q}\left(\boldsymbol{\theta}_{i}-\boldsymbol{\mu}_{\theta}-\boldsymbol{D} \boldsymbol{z}_{i}\right)+\frac{2}{\nu}\right\}^{-1},
$$

com $\boldsymbol{z}_{i}>\mathbf{0}$ e $\nu>2$.

\section{A.10 Condicional Completa de $\delta$ : Caso Unidimensio- nal}

No modelo Kd3PP-St a condicional completa para o vetor de parâmetros associado à assimetria $\boldsymbol{\delta}$ só possui forma fechada (distribuição conhecida) no caso particular unidimensional, ou seja, no caso em que $K=1$. Pois, a partir da distribuição a priori (4.3) observamos que

$$
\begin{aligned}
\theta_{i} \mid z_{i}, \mu_{\theta}, \sigma_{\theta}, \delta, \omega_{i} & \sim N_{1}\left(\mu_{\theta}+\delta Z_{i}, \frac{\sigma_{\theta}^{2}}{\omega_{i}}\right) \\
\delta & \sim N_{1}\left(0, \gamma_{0}\right),
\end{aligned}
$$

de modo que esta estrutura pode ser reescrita como um modelo de regressão linear normal/normal heterocedástico, ou seja:

$$
\begin{aligned}
\theta_{i}^{*} & =Z_{i} \delta+\epsilon_{i}, \text { com variáveis independentes } \\
\epsilon_{i} & \sim N\left(0, \frac{\sigma_{\theta}^{2}}{\omega_{i}}\right), \quad i=1, \ldots, N, \mathrm{e} \\
\delta & \sim N_{1}\left(0, \gamma_{0}\right),
\end{aligned}
$$

em que $\theta_{i}^{*}=\theta_{i}-\mu_{\theta}$.

A partir desta representação do modelo é possível concluir que

$$
\delta \mid \ldots \sim N_{1}\left(\Psi_{\delta} \psi_{\delta}, \Psi_{\delta}\right)
$$

em que

- $\Psi_{\delta}=\left(\gamma_{0}^{-1}+\boldsymbol{z}^{\prime} \Sigma_{\delta}^{-1} \boldsymbol{z}\right)^{-1}$,

$$
\operatorname{com} \boldsymbol{z}=\left(z_{1}, \ldots, z_{N}\right)^{\prime} \quad \text { e } \quad \Sigma_{\delta}^{-1}=\frac{1}{\sigma_{\theta}^{2}} \operatorname{diag}\left(\omega_{1}, \ldots, \omega_{N}\right) .
$$

Matos, G. S. 
- $\psi_{\delta}=\gamma_{0}^{-1} .0+\boldsymbol{z}^{\prime} \Sigma_{\delta}^{-1} \boldsymbol{\theta}^{*}=\boldsymbol{z}^{\prime} \Sigma_{\delta}^{-1} \boldsymbol{\theta}^{*}$, $\operatorname{com} \boldsymbol{\theta}^{*}=\left(\theta_{1}-\mu_{\theta}, \ldots, \theta_{N}-\mu_{\theta}\right)^{\prime}=\boldsymbol{\theta}-\mu_{\theta} \mathbf{1}_{N}$. 
Apêndice B

\section{Função MCMCirtKd2PPSn para a estimação do modelo Kd2PPSn}

Neste Apêndice apresentamos algumas informações sobre a função utilizada na estimação dos modelos analisados no Capítulo 5. Esta função, aqui denominada MCMCirtKd2PPSn, foi escrita em $R$ e C++ a partir de adequadas modificações da função $M C M C i r t K d$ do pacote MCMCpack (Martin; Quinn, 2006) do ambiente computacional e estatístico (programa) R (R Development Core Team, 2007). Inicialmente, esta função será disponibilizada aos usuários $R$ que realizarem os seguintes procedimentos:

1. Solicitar ao autor deste trabalho o arquivo NAMESPACE e os arquivos de extensão .R e .cc necessários à compilação da função. Para isto, recomendamos que tal solicitação seja enviada ao:

E-mail: gsmatos@gmail.com

Título: Solicito arquivos da função MCMCirtKd2PPSn

2. Compilar os arquivos que tornam a função disponível para o usuário R; conforme orientação encontrada no manual de desenvolvedores* de funções para o pacote MCMCpack (Martin; Quinn, 2006) do programa R (ver também algumas orientações descritas na Sub-seção 4.2.4.5).

A seguir descrevemos a função MCMCirtKd2PPSn que estará sendo continuamente testada e ampliada.

* Disponível em: http://mcmcpack.wustl.edu/wiki/index.php/MCMCpack_Development_Page. 
MCMCirtKd2PPSn Markov Chain Monte Carlo for K-Dimensional Item Response

Theory Model with 2 Parameter Probit Link and Skew-normal Distribution for Latent Traits

\section{Description}

This function generates a sample from the posterior distribution of a K-dimensional item response theory (IRT) model, with flexible skew-normal priors on the subject abilities (ideal points), and normal priors on the item parameters. The user supplies data and priors, and a sample from the posterior distribution is returned as an mcmc object, which can be subsequently analyzed with functions provided in the coda package.

\section{Usage}

MCMCirtKd(datamatrix, dimensions, item.constraints=list(), burnin $=1000, \operatorname{mcmc}=10000$, thin=1, verbose $=0$, seed $=\mathrm{NA}$, alphabeta. start $=\mathrm{NA}, \mathrm{bO}=0, \mathrm{~B} 0=0$, store.item $=$ FALSE, store.ability=TRUE, drop. constant. items=TRUE, ... )

MCMCirtKd2PPSt (datamatrix, dimensions, item.constraints=list(), burnin=1000, mcmc=10000, thin=1, tune. Skew=NA, tune.mean.df=2.001, tune. variance. $d f=1$, verbose $=0$, seed $=N A$, theta.start=NA, alphabeta.start $=\mathrm{NA}$, beta3.start=NA, SigmaInv.start=NA, mutheta.start=NA, skew. start $=\mathrm{NA}$, df.tstart $=\mathrm{NA}$, $\mathrm{b} 0=0, \mathrm{~B} 0=0, \mathrm{~b} 30=\mathrm{NA}, \mathrm{df} . \mathrm{Q} 0=\mathrm{NA}, \mathrm{SigmaQ0}=\mathrm{NA}$, $\mathrm{mu} 0=0, \mathrm{SInv0}=0$, SigmaInvdelta0 $=0$, store.item=TRUE, store.ability=TRUE, store.SigmaInv=FALSE, store. Mutheta=FALSE, drop. constant . items=TRUE, model="Kd2PPN", ...)

\section{Arguments}

datamatrix The matrix of data. Must be 0,1 , or missing values. It is of dimensionality subjects by items.

Matos, G. S.

IME/USP 
dimensions The number of dimensions in the latent space.

item. constraints

List of lists specifying possible equality or simple inequality constraints on the item parameters. A typical entry in the list has one of three forms: rowname=list $(\mathrm{d}, \mathrm{c})$ which will constrain the dth item parameter for the item named rowname to be equal to c, rowname=list (d, "+") which will constrain the dth item parameter for the item named rowname to be positive, and rowname=list $(\mathrm{d}$, -") which will constrain the dth item parameter for the item named rowname to be negative. If $\mathrm{x}$ is a matrix without row names defaults names of "V1", "V2", .., , etc will be used. In a $\mathrm{K}$ dimensional model, the first item parameter for item $i$ is the difficulty parameter $\left(\alpha_{i}\right)$, the second item parameter is the discrimation parameter on dimension $1\left(\beta_{i, 1}\right)$, the third item parameter is the discrimation parameter on dimension $2\left(\beta_{i, 2}\right), \ldots$, and the $(\mathrm{K}+1)$ th item parameter is the discrimation parameter on dimension $\mathrm{K}\left(\beta_{i, 1}\right)$. The item difficulty parameters $(\alpha)$ should generally not be constrained.

burnin The number of burn-in iterations for the sampler.

mcmc The number of iterations for the sampler.

thin The thinning interval used in the simulation. The number of iterations must be divisible by this value.

tune.Skew The tuning parameter for the Metropolis-Hastings sampling of the multidimensional skewness parameter. NA(default): diagonal matriz with elements equal to $0.05 / 2$. Can be either a scalar or a $k$-vector. Must be strictly positive. If dimensions $=1$ it will not be used because a closed form exists for the complete conditional distribution to sample from skewness parameter.

tune.mean.df

The tuning parameter for the Metropolis-Hastings sampling of the degrees of freedom of a distribution to be defined in future versions (Not implemented!).

tune.variance.df

Maybe a second tuning parameter for the Metropolis-Hastings sampling of the degrees of freedom of a distribution to be defined in future versions (Not implemented!).

verbose A switch which determines whether or not the progress of the sampler is printed to the screen. If verbose is greater than 0 then every verboseth iteration will be printed to the screen.

seed The seed for the random number generator. If NA, the Mersenne Twister generator is used with default seed 12345; if an integer is passed

Matos, G. S.

IME/USP 
it is used to seed the Mersenne twister. The user can also pass a list of length two to use the L'Ecuyer random number generator, which is suitable for parallel computation. The first element of the list is the L'Ecuyer seed, which is a vector of length six or NA (if NA a default seed of $\operatorname{rep}(12345,6)$ is used). The second element of list is a positive substream number. See the MCMCpack specification for more details.

theta.start The starting values for $\boldsymbol{\theta}_{j}$. NA(default): 0 for all $\boldsymbol{\theta}_{j} /$ "random": sample from standard normal distribution / "eigen": eigen method.

alphabeta.start

The starting values for the $\alpha$ and $\beta$ difficulty and discrimination parameters. If alphabeta.start is set to a scalar the starting value for all unconstrained item parameters will be set to that scalar. If alphabeta.start is a matrix of dimension $(K+1) \times$ items then the alphabeta.start matrix is used as the starting values (except for equality-constrained elements). If alphabeta.start is set to NA (the default) then starting values for unconstrained elements are set to values generated from a series of proportional odds logistic regression fits, and starting values for inequality constrained elements are set to either 1.0 or -1.0 depending on the nature of the constraints.

beta3.start The starting values for $\beta_{3 j}$ (Is in testing phase!). NA(default): 0.2 for all $\beta_{3 j}$.

SigmaInv.start

The starting values for inverse of scale matrix $\boldsymbol{\Sigma}_{\theta}$ of latent traits distribution. May be a scalar (NA(default): 1) or a square matrix with the the same dimension of latent traits. Elements must be strictly positive. mutheta.start

The starting values for mean vector $\boldsymbol{\mu}_{\theta}$ of latent traits distribution. May be a scalar (NA(default): 0) or a vector with the the same dimension of latent traits.

skew.start The starting values for skewness vector $\boldsymbol{\delta}$ of latent traits distribution. May be a scalar (NA(default): 0) or a vector with the the same dimension of latent traits.

df.tstart The starting values for degrees of freedom of latent traits prior tdistribution (Is in testing phase!). NA(default): dimensions +0.001 .

b0 The prior means of the $\alpha$ and $\beta$ difficulty and discrimination parameters, stacked for all items. If a scalar is passed, it is used as the prior mean for all items.

BO

The prior precisions (inverse variances) of the independent normal prior on the item parameters. Can be either a scalar or a matrix of dimension $(K+1) \times$ items.

Matos, G. S.

$\mathrm{IME} / \mathrm{USP}$ 
b30

The prior parameters of the beta prior guessing parameters $\beta_{3 j}$ (Is in testing phase!). May be NA(default): non-informative $\operatorname{Beta}\left(a_{j}=1, b_{j}=\right.$ 1 ), a vector with alternative values for $a_{j}$ and $b_{j}$ parameters or a matrix $J$ by 2 with Beta distribution parameters for all itens.

df .Q0 The prior degree of freedom of the Wishart prior distribution of inverse of scale matrix $\Sigma_{\theta}$ of latent traits distribution. May be NA(default): dimensions +1 or a scalar df.Q0 > dimensions.

SigmaQ0 The prior positive definitive matrix of the Wishart prior distribution of inverse of scale matrix $\boldsymbol{\Sigma}_{\theta}$ of latent traits distribution. Can be either a scalar (NA(default): 1) or a matrix of dimension $K \times K$.

mu0 The prior vector mean of mean vector $\boldsymbol{\mu}_{\theta}$ of latent traits distribution. May be a scalar (NA(default): 0) or a vector with the the same dimension of latent traits.

SInv0 The prior precision matrix of mean vector $\boldsymbol{\mu}_{\theta}$ distribution of latent traits. May be a scalar (NA(default): 1) or a square matrix with the the same dimension of latent traits. Elements must be strictly positive.

SigmaInvdelta0

The prior precision matrix of skewness vector $\boldsymbol{\delta}$ distribution of latent traits. May be a scalar (NA(default): 1) or a square matrix with the the same dimension of latent traits. Elements must be strictly positive.

store.item A switch that determines whether or not to store the item parameters for posterior analysis. NOTE: In applications with many items this takes an enormous amount of memory. If you have many items and want to want to store the item parameters you may want to thin the chain heavily. By default, the item parameters are not stored.

store.ability

A switch that determines whether or not to store the subject abilities for posterior analysis. NOTE: In applications with many subjects this takes an enormous amount of memory. If you have many subjects and want to want to store the ability parameters you may want to thin the chain heavily. By default, the ability parameters are all stored.

store.SigmaInv

A switch that determines whether or not to store the scale, covariance and correlation matrix parameters of the latent trait distribution for posterior analysis. By default, these matrix parameters are not stored and in this case are not estimated as they are fixed in an identitie matrix.

store.Mutheta

A switch that determines whether or not to store the location and

Matos, G. S.

IME/USP 
expected value vector parameter of the latent trait distribution for posterior analysis. By default, these parameters are not stored and in this case are not estimated as they are fixed in null vector.

drop. constant.items

A switch that determines whether or not items that have no variation should be deleted before fitting the model. Default $=$ TRUE.

model Specify the multidimensional IRT model indicating the number of parameters of item response function and the latent trait distribution to sample from posterior distribution. May be a character choosing from the following options: "Kd1PPN" (all discriminations are setup to 1), "Kd2PPN" (default), "Kd3PPN" (Is in testing phase!), "Kd1PPSn" (all discriminations are setup to 1), "Kd2PPSn", "Kd3PPSn" (Is in testing phase!), "Kd1PPSt" (not implemented!), "Kd2PPSt"(not implemented!), "Kd3PPSt" (not implemented!).

... $\quad$ further arguments to be passed

\section{Details}

MCMCirtKd2PPSn simulates from the posterior distribution using standard Gibbs sampling using data augmentation (a normal draw for the subject abilities, a multivariate normal draw for the item parameters, and a truncated normal draw for the latent utilities). The simulation proper is done in compiled $\mathrm{C}++$ code to maximize efficiency. Please consult the coda documentation for a comprehensive list of functions that can be used to analyze the posterior sample.

The default number of burnin and momc iterations is much smaller than the typical default values in MCMCpack. This is because fitting this model is extremely computationally expensive. It does not mean that this small of a number of scans will yield good estimates. The priors of this model need to be proper for identification purposes. The user is asked to provide prior means and precisions (not variances) for the item parameters and the subject parameters.

The model takes the following form. We assume that each subject has an ability (ideal point) denoted $\theta_{j}(K \times 1)$, and that each item has a difficulty parameter $\alpha_{i}$ and discrimination parameter $\beta_{i}(K \times 1)$. The observed choice by subject $j$ on item $i$ is the observed data matrix which is $(I \times J)$. We assume that the choice is dictated by an unobserved utility:

$$
z_{i, j}=-\alpha_{i}+\beta_{i}^{\prime} \theta_{j}+\varepsilon_{i, j}
$$

Where the $\varepsilon_{i, j} \mathrm{~S}$ are assumed to be distributed as some of asymmetrical distribution of Sahu, Dey and Branco (2003) not necesseraly in a standard form. The parameters 
of interest are the subject abilities (ideal points), the item parameters and/or some parameters of latent trait distribution.

We assume the following priors. For the subject abilities (ideal points) we assume independent Skew normal priors:

$$
\boldsymbol{\theta}_{j} \sim \operatorname{Sn}\left(\boldsymbol{\mu}_{\theta}, \boldsymbol{\Sigma}_{\theta}, \boldsymbol{\delta}\right)
$$

For each item parameter, we assume independent normal priors:

$$
\left[\alpha_{i}, \beta_{i}\right]^{\prime} \sim \mathcal{N}_{(K+1)}\left(b_{0, i}, B_{0, i}\right)
$$

Where $B_{0, i}$ is a diagonal matrix. One can specify a separate prior mean and precision for each item parameter.

The model is identified by the constraints on the item parameters (see Jackman 2001, Bégin and Glas 2001, Rivers 2003 and Matos 2008). The user cannot place constraints on the subject abilities. This identification scheme differs from that in MCMCirt1d, which uses constraints on the subject abilities to identify the model. In our experience, using subject ability constraints for models in greater than one dimension does not work particularly well.

As is the case with all measurement models, make sure that you have plenty of free memory, especially when storing the item parameters.

\section{Value}

An mcmc object that contains the posterior sample. This object can be summarized by functions provided by the coda package.

\section{References}

James H. Albert. 1992. "Bayesian Estimation of Normal Ogive Item Response Curves Using Gibbs Sampling." Journal of Educational Statistics. 17: 251-269.

Joshua Clinton, Simon Jackman, and Douglas Rivers. 2004. "The Statistical Analysis of Roll Call Data."American Political Science Review. 98: 355-370.

Simon Jackman. 2001. "Multidimensional Analysis of Roll Call Data via Bayesian Simulation." Political Analysis. 9: 227-241.

Valen E. Johnson and James H. Albert. 1999. Ordinal Data Modeling. Springer: New York.

Matos, G. S.

IME/USP 
Béguin, A. A. and Glas, C. A. W. (2001). MCMC estimation and some model-fit analysis of multidimensional IRT models. Psychometrika, v. 66, n. 4, p. 541-562.

Daniel Pemstein, Kevin M. Quinn, and Andrew D. Martin. 2007. Scythe Statistical Library 1.0. http://scythe.wustl.edu.

Martyn Plummer, Nicky Best, Kate Cowles, and Karen Vines. 2002. Output Analysis and Diagnostics for $M C M C$ (CODA). http://www-fis.iarc.fr/coda/.

Matos, G. S. 2008. "Modelos multidimensionais da TRI com distribuições assimétricas para os traços latentes." Tese de Doutorado em Estatística IME/USP. São Paulo - SP - Brazil.

Douglas Rivers. 2003. "Identification of Multidimensional Item-Response Models." Stanford University, typescript.

\section{See Also}

plot.mcmc, summary.mcmc, MCMCirt1d, MCMCordfactanal

\section{Examples}

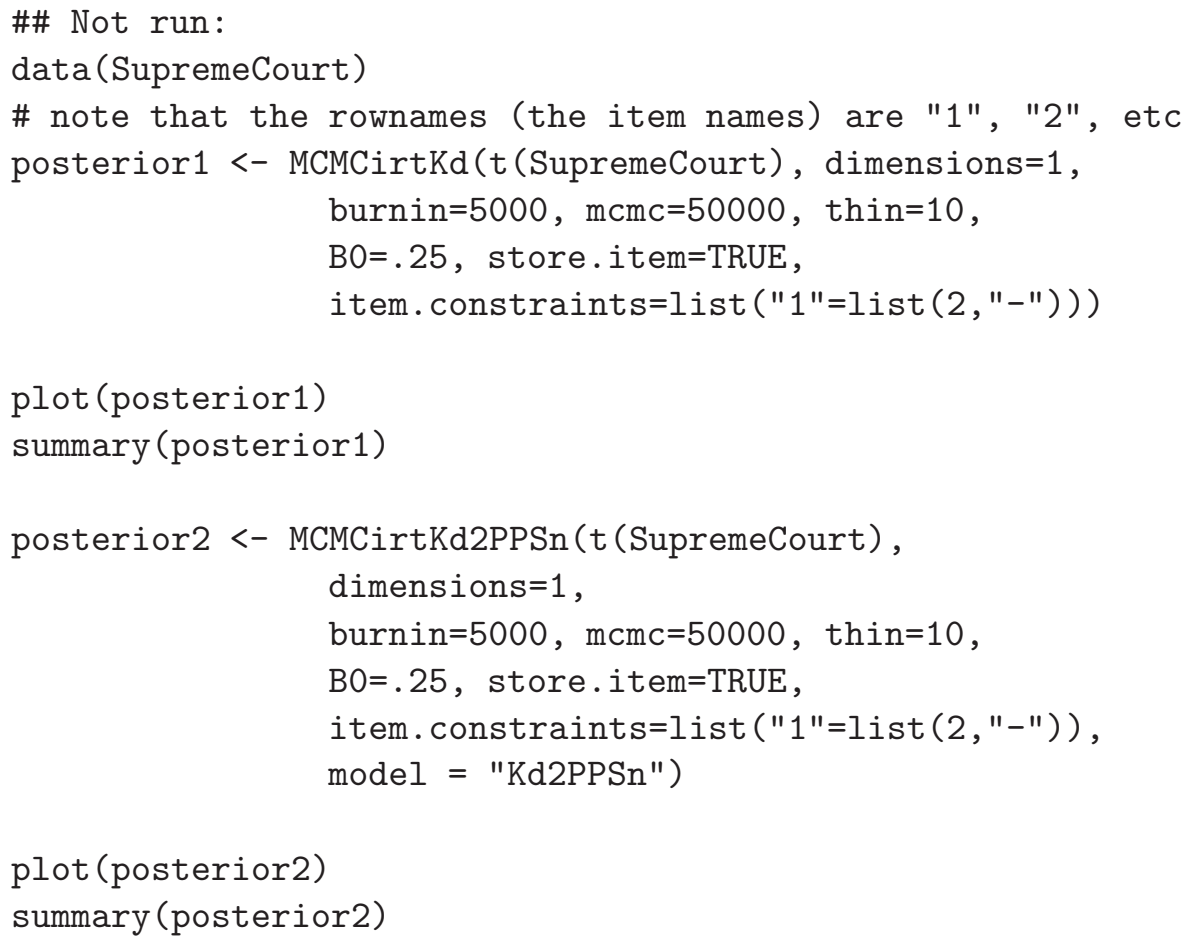

Matos, G. S. 


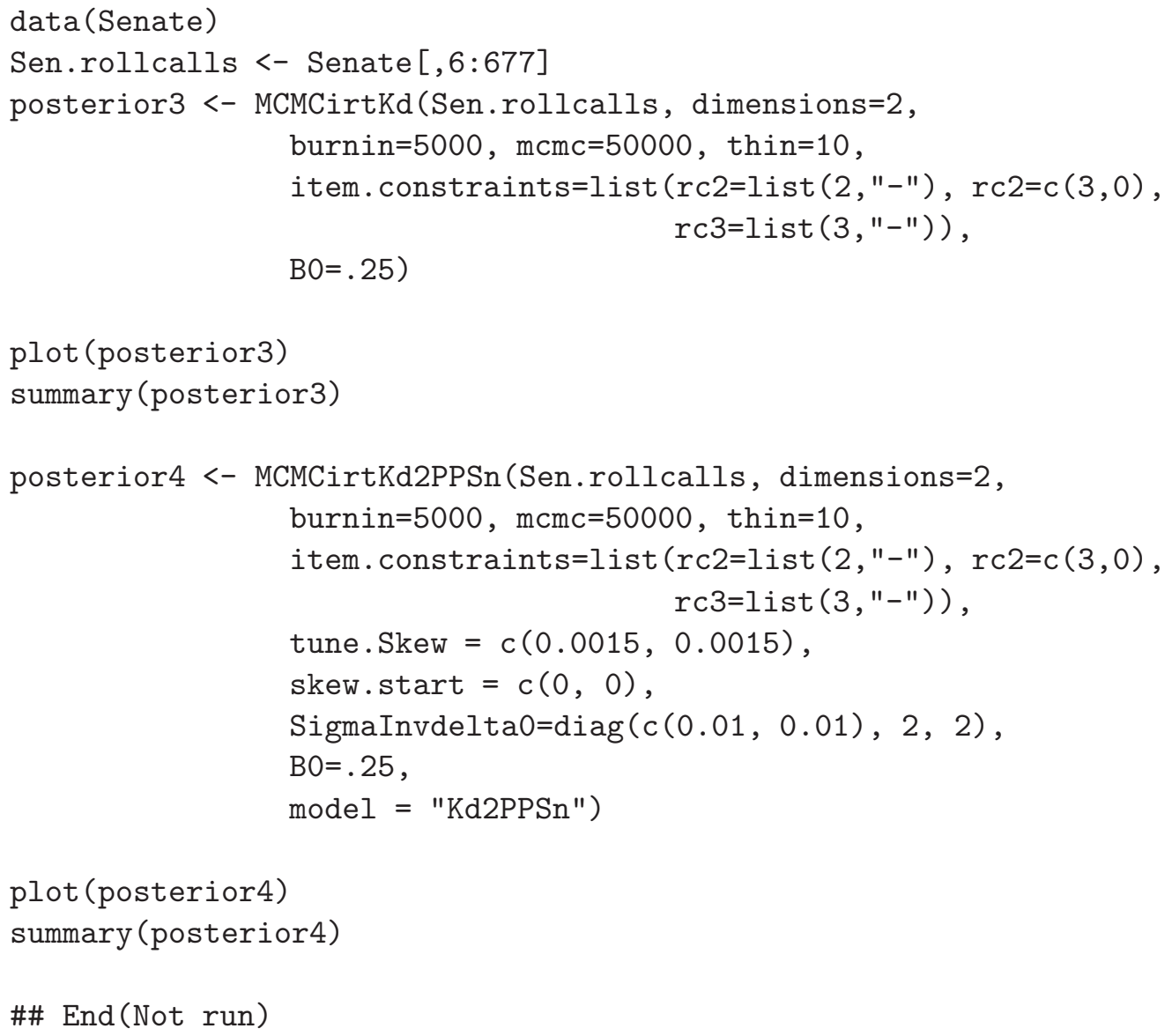

Matos, G. S. 


\section{Referências Bibliográficas}

Adams, R. J.; Wilson, M. and Wang, W. chung. (1997). The Multidimensional Random

Coefficients Multinomial Logit Model. Applied Psychological Measurement, v. 21, n. 1, p. $1-23$.

Albert, J. H. (1992). Bayesian estimation of normal ogive item response curves using gibbs sampling. Journal of Educational Statistics, v. 17, p. 251-269.

Albert, J. H. and Chib, S. (1993). Bayesian analysis of binary and polychotomous response data. Journal of American Statistical Association, v. 88, n. 422, p. 669-679.

Algina, J. (1980). A note on identification in the oblique and orthogonal factor analysis models. Psychometrika, v. 45, n. 3, p. 393-396.

Andersen, E. B. (1980). Discrete statistical models with social science applications. [S.l.]: Amsterdam; New York : North-Holland Pub. Co.: sole distributors for the U.S.A. and Canada, American Elsevier Pub. Co.

Andrade, D. F. (2001). Comparando desempenhos de grupos de alunos por intermédio da teoria da resposta ao item. Estudos em Avaliação Educacional, São Paulo,, v. 23, p. 31-70.

Andrade, D. F.; Tavares, H. R. and Valle, R. C. (2000). Teoria da Resposta ao Item: Conceitos e Aplicações. [S.l.]: 14o. SINAPE: Caxambú - MG.

Arellano-Valle, R. B.; Branco, M. D. and Genton, M. (2006). A unified view on skewed distributions arising from selections. The Canadian Journal of Statistics, v. 34, n. 4, p. $1-21$.

Azevedo, C. L. N. Métodos de estimação na Teoria de Resposta ao Item. 2003. Dissertação (Mestrado) - IME/USP, São Paulo - SP.

Azevedo, C. L. N. Modelos longitudinais de grupos múltiplos multiníveis na teoria da resposta ao item: métodos de estimação e seleção estrutural sob uma perspectiva bayesiana. 2008. Tese (Doutorado) — IME/USP, São Paulo - SP. 
Azzalini, A. (1985). A class of distributions which includes the normal ones. Scand. J. Statistical, v. 12, p. 171-178.

Azzalini, A. and Capitanio, A. (1999). Statistical applications of the multivariate skew normal distribution. Journal of the Royal Statistical Society Series B, v. 61, p. 579-602.

Azzalini, A. and Dalla Valle, A. (1996). The multivariate skew-normal distribution. Biometrika, v. 8, p. 715-726.

Bailey, M. (2001). Ideal point estimation with a small number of votes: a random-effects approach. Political Analysis, v. 9, n. 3, p. 192-210. Disponível em: $<$ http://polmeth.wustl.edu/polanalysis.php $>$.

Baker, F. B. (1998). An investigation of the item parameter recovery characteristics of a Gibbs sampling approach. Applied Psychological Measurement, v. 22, p. 153-169.

Baker, F. B. and Kim, S. (2004). Item Response Theory - Parameter Estimation Techniques. [S.l.]: 2nd Ed., Revised and Expanded. New York: Marcel Dekker, Inc.

Bartholomew, D. J. (1999). Latent Variable Models and Factor Analysis. [S.l.]: New York: Oxford University Press (2th ed.).

Bazán, J. L. Modelos de Resposta ao Item Assimétricos. 2005. Tese (Doutorado) IME/USP, São Paulo - SP.

Bazán, J. L.; Branco, M. D. and Bolfarine, H. (2006). A skew item response model. Bayesian Analysis,, v. 1, n. 4, p. 861-892.

Bitar, H. A. F. et al. (1998). O sistema de avaliação de rendimento escolar do estado de são paulo: implantação e continuidade. Publicação: Série Idéias n. 30. São Paulo: FDE, 1998, p. 9-20. Disponivel em: http://www.crmariocovas.sp.gov.br/ext_a.php?t=006.

Bock, R. D. (1972). Estimating item parameters and latent ability when responses are scored in two or more nominal categories. Psychometrika, v. 46, p. 443-459.

Bock, R. D. and Aitkin, M. (1981). Marginal maximum likelihood estimation of item parameters: An application of a EM algorithm. Psychometrika, v. 46, p. 433-459.

Bock, R. D.; Gibbons, R. and Muraki, E. (1988). Full-Information item factor analysis. Applied Psychological Measurement, v. 12, n. 3, p. 261-280.

Bock, R. D. et al. (2003). TESTFACT 4.0 [Computer software and manual]. [S.l.]. Scientific Software International, Inc., Lincolnwood, Illinois.

Bock, R. D. and Lieberman, M. (1970). Fitting a response model for $n$ dichotomously scored itens. Psychometrika, v. 35, p. 179-197. 
Bock, R. D. and Zimowski, M. F. (1997). Multiple Group IRT. [S.l.]: In Handbook of Modern Item Response Theory. W. J. van der Linden \& R. K. Hambleton Eds. New York: Springer-Verlag.

Bortolotti, S. Aplicação de um Modelo de Desdobramento da Teoria da Resposta ao Item - TRI. 2003. Dissertação (Dissertação de Mestrado) - EPS/UFSC, Santa Catarina $\mathrm{SC}$.

Bortolotti, S. L. V. and Andrade, D. F. (2007). Aplicação de um modelo de desdobramento graduado generalizado - GGUM da teoria da resposta ao item. Estudos em Avaliação Educacional.

Bowden, R. (1973). The theory of parametric identification. Econometrica, v. 41, p. 1069-1074.

Box, G. and Tiao, G. (1973). Bayesian inference in statistical analysis. [S.l.]: Reading, MA: Addison-Wesley.

Branco, M. D. and Arellano-Valle, R. (2004). Distribuições Elípticas Assimétricas. [S.l.]: 16o. SINAPE - 26 a 30/07/04 ABE - Associação Brasileira de Estatística.

Brooks, S. P. (2002). Discussion on the paper by Spiegelhalter, Best, Carlin and van der Linde. Journal Royal Statistical Society, Series B, v. 64, n. 3, p. 616-618.

Béguin, A. A. and Glas, C. A. W. (2001). Mcmc estimation and some model-fit analysis of multidimensional irt models. Psychometrika, v. 66, n. 4, p. 541-562.

Carlin, B. P. and Louis, T. A. (1996). Bayes and Empirical Bayes Methods for Data Analysis. [S.l.]: Chapman \& Hall.

Casella, G. and George, E. I. (1992). Explaining the Gibbs Sampler. The American Statistician,, v. 46, n. 3, p. 167-174.

Chang, H.-H. (1996). The asymptotic posterior normality of the latent trait for polytomous IRT models. Psychometrika, v. 61, n. 3, p. 445-463.

Chang, H.-H. and Stout, W. (1993). The asymptotic posterior normality of the latent trait in an IRT model. Psychometrika, v. 58, n. 1, p. 37-52.

Chen, M.-H.; Shao, Q.-M. and Ibrahim, J. G. (2000). Monte Carlo Methods in Bayesian Computation. [S.l.]: Spinger-Verlag. New York, Inc.

Chib, S. (2000). Bayesian methods for correlated binary data. In Dey, D. K., Gosh, S. K. $\& 3$ Mallick, B. K., Generalized Linear Models: A bayesian perspective (p. 113-131). [S.l.]: Marcel Dekker, Inc. 
Chib, S. and Greenberg, E. (1995). Understanding the Metropolis-Hastings Algorithm. The Americam Statistician,, v. 49, n. 4, p. 327-335.

Christoffersson, A. (1975). Factor analysis of dichotomized variables. Psychometrika, v. 40 , p. $5-22$.

Clinton, J.; Jackman, S. and Rivers, D. (2004). The statistical analysis of roll call data. American Political Science Review, v. 98, p. 355-370.

Cordeiro, G. M. (1992). Introdução á Teoria de Verossimilhança. [S.l.]: 10 Simpósio Nacional de Probabilidade e Estatística.

Cúri, M. Análise de questionários com itens constrangedores. 2006. Tese (Doutorado) IME/USP, São Paulo - SP.

Dawid, A. P. (1979). Conditional Independence in Statistical Theory (with discussion). Journal of the Royal Statistical Society, Series B, v. 41, p. 1-31.

De Ayala, R. J. and Sava-Bolesta, M. (1999). Item parameter recovery for the nominal response model. Applied Psychological Measurement, v. 23, n. 1, p. 3-19.

de la Torre, J. and Patz, R. J. (2002). A multidimensional item response theory approach to simultaneous ability estimation. Educational Resources Information Center (ERIC). Disponível em: <http://www.eric.ed.gov/>.

DeMars, C. E. (2003). Sample size and the recovery of nominal response model item parameters. Applied Psychological Measurement, v. 27, n. 4, p. 275-288.

Dempster, A. P.; Laird, N. M. and Rubin, D. B. (1977). Maximum likelihood from incomplete data via the em algorithm (with discussion). Journal of the Royal Statistical Society, Series B, v. 39, p. 1-38.

Douglas, J. (1997). Joint consistency of nonparametric item characteristic curve and ability estimation. Psychometrika, v. 62, n. 1, p. 7-28.

Dréze, J. H. (1974). Bayesian theory of identification in simultaneous equations models. [S.l.]: In S.E. Fienberg \& A. Zellner (eds.), Studies in Bayesian Econometrics and Statistics, pp. 159-174. Amsterdam: North-Holland.

Dréze, J. H. and Richard, J.-F. (1983). Bayesian analysis of simultaneous equation systems. [S.l.]: In Z. Griliches \& M.D. Intriligator (eds.), Handbook of Econometrics 2, pp. 517-600. Amsterdam: North-Holland.

Fischer, G. H. and Molenaar, I. W. (1995). Rasch Models Foundations, Recents Developments and Applications. [S.l.]: Springer, Berlim. 
Flury, B. and Zoppé, A. (2000). Exercises in EM. The American Statistician,, v. 54, n. 3, p. 207-209.

Fox, J. P. (2005). Multilevel IRT using dichotomous and polytomous items. British Journal of Mathematical and Statistical Psychology,, v. 58, p. 145-172.

Fraser, C. (1988). NOHARM: A computer program for fitting both unidimensional and multidimensional normal ogive models of latent trait theory. [S.l.]: Armidale, Australia: University of New England.

Froelich, A. G. Assesing unidimensionality of test item and asymptotics of parametric item response theory. 2000. Tese (Unpublished doctoral dissertation) University of Illnois at Urbana-Champaign, Departament of Statistics. Disponível em: $<$ http://www.public.iastate.edu/ amyf/>.

Gamerman, D. and Lopes, H. F. (2006). Markov Chain Monte Carlo: Stochastic Simulation for Bayesian Inference. [S.l.]: London: Chapman \& Hall.

Gelfand, A. E. (1996). Model determination using sampling based methods. In Gilks, W. R., Richardson, S. and Spiegelhalter, D. J. (Eds.). Markov chain Monte Carlo in Practice. [S.l.]: London: Chapman \& Hall, pag. 145-161.

Gelfand, A. E. and Sahu, S. K. (1999). Identifiability, improper priors and Gibbs sampling for generalized linear models. Journal of the American Statistical Association, v. 94 , n. 445 , p. $247-253$.

Gelfand, A. E. and Smith, A. F. M. (1990). Sampling-based approaches to calculating marginal densities. Journal of American Statistical Association, v. 85, p. 398-409.

Gelman, A. et al. (1995). Bayesian data analysis. [S.l.]: London: Chapman and Hall.

Gelman, A.; Roberts, G. O. and Gilks, W. R. (1996). Efficient Metropolis jumping rules. In J. M. Bernardo, J.O. Berger, A.P. Dawid and A.F.M. Smith (Eds.). [S.1.]: Bayesian Statistics 5: Proceedings of the Fifth Valencia International Meeting (pp. 599-608). New York: Oxford.

Geman, S. and Geman, D. (1984). Stochastic relaxation, gibbs distribution and the bayesian restoration of images. IEEE Transactions on Pattern Analysis and Machine Intelligence, v. 6, p. 721-741.

Genton, M. G. (2004). Skew-Elliptical Distributions and their Applications: A Journey Beyond Normality. [S.l.]: Chapman \& Hall/CRC.

Geweke, J. (1992). Evaluating the accuracy of sampling-based approaches to the calculation of posterior moments (with discussion). In Bayesian Statistics 4 (eds J. M. Bernardo et al.). Oxford University Press, Oxford, p. 169-193.

Matos, G. S.

IME/USP 
Geweke, J. F. and Singleton, K. J. (1980). Evaluating the accuracy of sampling-based approaches to the calculation of posterior moments (with discussion).interpreting the likelihood ratio statistics in factor models when sample size is small. J. Amer. Statist. Assoc., v. 75, p. 133-137.

Ghosh, M. et al. (2000). Bayesian estimation for item response models. Journal of Statistical Planning and Inference, v. 88, p. 99-115.

Gilks, W. R.; Best, N. G. and Tan, K. K. C. (1995). Adaptive rejection metropolis sampling within gibbs sampling. Applied Statistics, p. 455-472.

Haberman, S. J. (1977). Maximum likelihood estimates in exponencial response models. Annals of Statistics, v. 5, p. 815-841.

Hambleton, R. K.; Swaminathan, H. and Rogers, H. J. (1991). Fundamentals of Item Response Theory. [S.l.]: Newbury Park: Sage Publications.

Harwell, M. et al. (1996). Monte Carlo studies in Item Response Theory. Applied Psychological Measurement, v. 20, n. 2, p. 101-125.

Heinen, T. (1996). Latent Class and Discrete Latent Trait Models: Similarities and Differences. [S.l.]: Advanced Quantative Techniques in the Social Sciences, Sage Publications, Thousans Oaks, CA.

Hobert, J. P. and Casella, G. (1996). The effect of improper priors on gibbs sampling in hierarchical linear mixed models. Journal of American Statistical Association, v. 91, n. 436 , p. $1461-1473$.

Holman, R. and Glas, C. A. W. (2005). Modellling non-ignorable missing-data mechanisms with item response theory models. British Journal of Mathematical and Statistical Psychology, v. 58, p. 1-17.

Jackman, S. (2000). Estimation and inference via bayesian simulation: an introduction to Markov Chain Monte Carlo. American Journal of Political Science, v. 44, n. 2, p. 369-398.

Jackman, S. (2001). Multidimensional analysis of roll call data via bayesian simulation: identification, estimation, inference and model checking. Political Analysis, v. 9, p. $227-241$.

Jöreskog, K. G. (1969). A general approach to confirmatory maximum likelihood likelihood factor analysis. Psychometrika, v. 34, n. 2, p. 183-202.

Kim, S. H. and Cohen, A. S. (1999, April). Accuracy of parameter estimation in Gibbs sampling under the two-parameter logistic model. Paper presented at the annual meeting of the American Educational Research Association, Montreal, Canada. 
Kolen, M. J. and Brennan, R. L. (1995). Test Equating - Methods and Practices. [S.l.]: New York: Springer.

Lancaster, T. (2000). The incidental parameter problem since 1948. Journal of Econometrics,, v. 95, p. 391-413.

Li, Y. H. and Schafer, W. D. (2005). Trait parameter recovery using multidimensional computerized adaptive testing in reading and mathematics. Applied Psychological Measurement, v. 29, p. 3-25.

Lindley, D. V. (1971). Bayesian Statistics: A Review. [S.l.]: Philadelphia, PA: SIAM.

Little, R. J. A. and Rubin, D. B. (1983). On jointly estimating parameters and missing data by maximizing the complete-data likelihood. The American Statistician, v. 37, p. 218-220.

Little, R. J. A. and Rubin, D. B. (1987). Statistical analysis with missing data. [S.l.]: John Wiley, New York.

Liu, J. S. (2001). Monte Carlo Strategies in Scientific Computing. [S.l.]: Springer-Verlag New York, Inc.

Lopes, H. F. and West, M. (2004). Bayesian model assesment in factor analysis. Statistica Sinica, v. 14, p. 41-67.

Lord, F. M. (1968). An analysis of the verbal scholastic aptitude test using Birnbaum's three-parameter logistic model. Educational and Psychological Measurement, v. 28 , p. $989-1020$.

Lord, F. M. (1975). Evaluation with artificial data of a procedure for estimating ability and item characteristic curve parameters. [S.l.]: (Research Bulletin RB-75-33). Princeton, NJ: Educational Testing Service.

Lord, F. M. (1980). Applications of Item Response Theory to Practical Testing Problems. [S.l.]: Hillsdale: Lawrence Erlbaum Associates, Inc.

Lord, F. M. and Novick, M. R. (1968). Statistical Theories of Mental Theory Test Scores. [S.l.]: Reading, MA.: Addison-Wesley.

Martin, A. D. and Quinn, K. M. (2002). Dynamic ideal point estimation via Markov Chain Monte Carlo for the U.S. Supreme Court, 1953-1999. Political Analysis, v. 10, n. 2, p. $134-153$.

Martin, A. D. and Quinn, K. M. (2006), Applied Bayesian Inference in R using MCMCpack. In: $R$ News. The Newsletter of the $R$ Project. [S.l.]: Martyn Plummer and Paul Murrell, (2006). p. 2-7. 
Matos, G. S. Teoria da Resposta ao Item: Uma Proposta de Modelo Multivariado. 2001. Dissertação (Mestrado) - Universidade Federal de Pernambuco (UFPE) - Departamento de Estatística (DE), Recife - PE.

McDonald, R. P. (1982). Linear versus nonlinear models in item response theory. Applied Psychological Measurement, v. 6, p. 379-396.

McDonald, R. P. (1997). Normal-Ogive multidimensional model. [S.l.]: In W. J. Linden \& R. K. Hambleton (Eds.), Handbook of modern item response theory (pp. 257-269). New York: Springer.

McDonald, R. P. (1997). Normal-Ogive Multidimensional Model. [S.l.]: In Handbook of Modern Item Response Theory. W. J. van der Linden \& R. K. Hambleton Eds. New York: Springer-Verlag.

McDonald, R. P. (1999). Test theory: A unified approach. [S.l.]: Mahwah, NJ: Lawrence Erlbaum.

Mellenbergh, G. J. (1994). Generalized linear item response theory. Psychological Bulletin, v. 115, n. 2, p. 300-307.

Mislevy, R. J. (1986). Bayes modal estimation in item response models. Psychometrika, v. 51 , p. $177-195$.

Mislevy, R. J. and Bock, R. D. (1985), Implementation of the EM algorithm in the estimations of item parameters: The BILOG computer program. In: D. J. Weiss (Ed.). Proceedings of the 1982 Item Response Theory and Computerized Adaptive Testing Conference. [S.l.]: Minneapolis, MN: University of Minnesota, Department of Psychology, Computerized Adaptive Testing Conference, (1985). p. 189-202.

Mislevy, R. J. and Chang, H. H. (2000). Does adaptive testing violate local independence? Psychometrika, v. 65, p. 149-156.

Mislevy, R. J. and Wu, P. K. (1996). Missing responses and IRT ability estimation: omits, choice, time limits, adaptive testing. ETS Research Report RR-96-30-ONR, Princeton, NJ: Educational Testing Service.

Neyman, J. and Scott, E. L. (1948). Consistent estimates based on partially consistent observations. Econometrica, v. 16, p. 1-32.

Nojosa, R. T. Modelos Multidimensionais para a Teoria da Resposta ao Item. 2001. Dissertação (Mestrado) - Universidade Federal de Pernambuco (UFPE) - Departamento de Estatística (DE), Recife - PE.

Nojosa, R. T. (2002). Teoria da resposta ao item(TRI) - modelos multidimensionais. Estudos em Avaliação Educacional,, v. 25, p. 123-166. 
O'Hagan, A. O. (1994). Kendall's Advanced Theory of Statistics: Bayesina Inference. [S.l.]: vol. 2B. New York: Halsted Press.

Pace, L. and Salvan, A. (1997). Principles of Statistical Inference. [S.l.]: Singapore: World Scientific in Advanced Series on Statistical Science \& Applied Probability, Vol. 4.

Patz, R. J. Markov Chain Monte Carlo methods for Item Response Theory Models with Applications for NAEP. 1996. Tese (Doctoral dissertation) - Department of Statistics, Carnegie Mellon University, Pittsburgh, Pennsylvania 15213.

Patz, R. J. and Junker, B. W. (1999a). A straightfoward approach to markov chain monte carlo methods for item response models. Journal of Educational and Behavioral Statistics, v. 24, p. 146-178.

Patz, R. J. and Junker, B. W. (1999b). Applications and extensions of MCMC in IRT: Multiple item types, missing data, and rated responses. Journal of Educational and Behavioral Statistics, v. 24, p. 342-366.

Plummer, M. et al. (2007). coda: Output analysis and diagnostics for MCMC. [S.l.]. R package version $0.12-1$.

Poirier, D. J. (1998). Revising beliefs in nonidentified models. Econometric Theory, v. 14, p. $483-509$.

Press, S. J. (2005). Applied multivariate analysis: Using bayesian and frequentist methods of inference. [S.l.]: 2th ed.. Dover publications, Inc.

Quinn, K. M. (2004). Bayesian factor analysis for mixed ordinal and continuos responses. Political Analysis, v. 12, n. 4, p. 338-353.

R Development Core Team. (2007). R: A Language and Environment for Statistical Computing. Vienna, Austria. ISBN 3-900051-07-0. Disponível em: $<$ http://www.R-project.org $>$.

Raftery, A. E. (1996). Hipothesis testing and model selection. In Gilks, W. R., Richardson, S. and Spiegelhalter, D. J. (Eds.). Markov chain Monte Carlo in Practice. [S.l.]: London: Chapman \& Hall, pag. 163-187.

Rasch, G. (1960/1980). Probabilistic models for some intelligence and attainment tests. [S.l.]: (Copenhagen, Danish Institute for Educational Research), expanded edition (1980) with foreword and afterword by B.D. Wright. Chicago: The University of Chicago Press.

Reckase, M. D. (1997). A linear logistic multidimensional model for dichotomous item response data. [S.l.]: In W. J. Linden \& R. K. Hambleton (Eds.), Handbook of modern item response theory (pp. 271-286). New York: Springer.

Matos, G. S.

IME/USP 
Reckase, M. D. (1997b). The past and future of multidimensional item response theory. Applied Psychological Measurement, v. 21, n. 1, p. 25-36.

Reckase, M. D. and Martineau, J. (2004). The vertical scaling of science achievement tests. Paper commissioned by the Committee on Test Design for K-12 Science Achievement Center for Education National Research Council.

Rijmen, F. et al. (2003). A nonlinear mixed model framework for item response theory. Psychological Methods, v. 8, n. 2, p. 185-205.

Rivers, D. (2003). Identification of multidimensional spatial voting models. Typescript. Department of Political Science, Stanford University.

Rizopoulos, D. (2006). ltm: An R package for latent variable modelling and item response theory analyses. Journal of Statistical Software, v. 17, n. 5, p. 1-25. Disponível em: <http://www.jstatsoft.org/v17>.

Roberts, J. S.; Donoghue, J. R. and Laughlin, J. E. (2000). A general item response theory model for unfolding unidimensional polytomous responses. Applied Psychological Measurement, v. 24, n. (1), p. 3-32.

Rothenberg, T. J. (1971). Identification in parametric models. Econometrica, v. 39, p. $577-591$.

Rubin, D. B. (1976). Inference and missing data. Biometrika, v. 63, p. 581-592.

Sahu, S. K. (2002). Bayesian estimation and model choice in item response theory. $J$. Statist. Comput. Simul., v. 72, n. 3, p. 217-232.

Sahu, S. K.; Dey, D. K. and Branco, M. D. (2003). A new class of multivariate skew distribution with applications to bayesian regression models. The Canadian Journal of Statistics, v. 31, n. 2, p. 129-150.

Seber, G. A. F. (1984). Multivariate observations. [S.l.]: Wiley, New York.

Segall, D. O. (1996). Multidimensional adaptive testing. Psychometrika, v. 61, n. 2, p. $331-354$.

Segall, D. O. (2002). Confirmatory Item Factor Analysis using Markov Chain Monte Carlo Estimation with Applications to Online Calibration in CAT. Paper presented at the annual meeting of the National Council on Measurement in Education (April, 2002).

Shao, J. and Tu, D. (1995). The Jackknife and Bootstrap. [S.l.]: Springer Series in Statistics.

Matos, G. S.

IME/USP 
Sinharay, S. (2004). Experiences with Markov Chain Monte Carlo convergence assesment in two psychometric examples. Journal of Educational and Behavioral Statistics, v. 29, n. 4 , p. $461-488$.

Skrondal, A. and Rabe-Hesketh, S. (2004). Generalized latent variable modeling: Multilevel, Longitudinal and Structural Equation Models. [S.l.]: Chapman \& Hall/CRC.

Spiegelhalter, D. J. et al. (2002). Bayesian measures of model complexity and fit. Journal Royal Statistical Society, Series B, v. 64, n. 3, p. 583-639.

Stout, W. F. (1990). A new item response theory modeling approach with applications to unidimensionality assessment and ability estimation. Psychometrika,, v. 55, p. 293-325.

Swaminathan, H. and Gifford, J. A. (1983). Estimation of parameters in the threeparameter latent trait model. [S.l.]: In D. Weiss (Ed.), New Horizonts in Testing. New York: Academic Press.

Takane, Y. and de Leeuw, J. (1987). On the relationship between item response theory and factor analysis of discretized variables. Psychometrika, v. 52, n. 3, p. 393-408.

Tanner, M. A. (1996). Tools for Statistical Inference: Methods for exploration of posterior distributions and likelihood functions. [S.l.]: Springer-Verlag New York, Inc.

Tanner, M. A. and Wong, W. H. (1987). The Calculation of Posterior Distributions by Data Augmentation. Journal of American Statistical Association, v. 82, n. 398, p. $528-550$.

Tavares, H. R. Teoria da resposta ao item para dados longitudinais. 2001. Tese (Doutorado) - IME/USP, São Paulo - SP.

te Marvelde, J. M. et al. (2006). Application of multidimensional item response theory models to longitudinal data. Educational and Psychological Measurement, v. 66, p. 5-34.

Tierney, L. (1994). Markov Chains for Exploring Posterior Distributions. The Annals of Statistics, v. 22, n. 4, p. 1701-1762.

Tsutakawa, R. K. and Johnson, J. C. (1990). The effect of uncertainty of item parameter estimation on ability estimates. Psychometrika, v. 55, n. 2, p. 371-390.

van der Linden, W. J. and Hambleton, R. K. (1997). Handbook of Modern Item Response Theory. [S.l.]: New York: Spring-Verlag.

van Dyk, D. A. and Meng, X.-L. (2001). The art of data augmentation. Journal of Computational and Graphical Statistics, v. 10, n. 1, p. 1-50. 
Wollack, J. A. et al. (2002). Recovery of Item Parameters in the Nominal Response Model: A Comparison of Marginal Maximum Likelihhod Estimation and Markov Chain Monte Carlo Estimation. Applied Psychological Measurement, v. 26, n. 3, p. 339-352.

Zimowski, M. F. et al. (1996). Bilog-MG: Multiple-group IRT analysis and test maintenance for binary items. Chicago, IL: Scientific Software International. 